0951 STEMA FUSERTA $11^{\circ} 31 \mathrm{~N}-150^{\circ} 31^{\circ} \mathrm{W}$ $04.05-65$ 0952 SulA dactylnTra $09^{\circ} 36 \mathrm{~h} /-151^{\circ} 43^{\prime} \mathrm{W} \quad 04-06-65$ 0953 Pafrimas parificus 09027N-151047í 04-16-65 0954 Puffenus paraticus $09^{\circ} 27^{\prime} \mathrm{N}-151^{\circ} 47 \mathrm{~W}$ 04-06-65

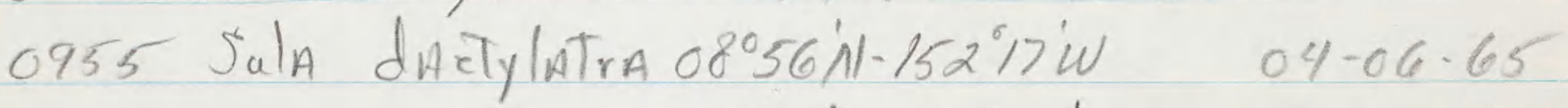
0956 $08^{\circ} 47 \mathrm{~A} 1-152^{\circ} 27^{\circ} \mathrm{W}$ $04-06.65$ 0957 $08^{\circ} \circ{ }^{\circ} \mathrm{N}-153^{\circ} 37 \mathrm{~W}$ $04-17-65$

0958 $08^{\circ} 07^{\prime} N-153^{\circ} 37 W$ $04-17 \cdot 65$ 0959 $08^{\circ} 09^{\prime} \mathrm{N}-153^{\circ} 43^{\circ} \mathrm{W}$ $04-07-65$

0961 $08^{\circ} 28^{\circ} \mathrm{N}-153^{\circ} 35^{\prime} \mathrm{W}$ 04.07 .65

0962 $08^{\circ} 28^{\prime} \mathrm{N}-153^{\circ} 35 \mathrm{~W}$ $0407-65$ 0963 STENA FUSCATA $16^{\circ} 07^{\prime} \mathrm{N}-161^{\circ} 34 \mathrm{~W}$ $04-11-65$ 0964 Phacthon rubricauda $16^{\circ} 08^{\mathrm{N}}-161 \mathrm{~S} 0^{\circ} \mathrm{W}$ $04-11 \cdot 65$ 0965 STEMA FUSCATA $16037 \mathrm{~N}-169^{\circ} 04 \mathrm{~W}$ $04-13-65$ 0966 Paffinas griesus $15^{\circ} 50^{\prime} \mathrm{N}-169^{\circ} 16^{\prime} \mathrm{W}$ $04-13-65$ 0967 Sula sula $13^{\circ} 30 \mathrm{~N}-169^{\circ} 38^{\mathrm{W}} \mathrm{W}$ $04-14.65$ 0968 SulA Sula $13^{\circ}, 4 \mathrm{~A} /-169^{\circ} 58^{\mathrm{W}} \mathrm{W}$ $04-14.65$ 0969 Sula diretylatira $15^{\circ} 24 \mathrm{~N}-169^{\circ} 5^{4} \mathrm{~W}$ $04-16 \cdot 65$ 0970 STERAA fUSCATA $15^{\circ} 30 \mathrm{~N}-170^{\circ} 80^{\circ} \mathrm{W}$ $04-16-65$ 0971 $15^{\circ} 27 \mathrm{~N}-170^{\circ} / 2 \mathrm{~W}$ $04-16-65$ 0972 $15^{\circ} 24 \mathrm{~N}-170^{\circ} 16 \mathrm{~W}$ $04-16-65$ 
0973 STERA FUSEATA $15^{\circ} 22^{\prime} \mathrm{Ml}-170^{\circ} 19^{\prime} \mathrm{W}$ 04-16-65 $0974 \quad 15^{\circ} 10^{\prime} \mathrm{N}-170^{\circ} 28^{\circ} \mathrm{W} \quad 04-16-65$ $0975 \quad " 15^{\circ} 10^{\prime} \mathrm{N}-120^{\circ} 28^{\circ} \mathrm{W} \quad 04-16.65$ 0976 SulA Suln $15^{\circ} 10 \mathrm{Al}-170^{\circ} 31^{\prime} \mathrm{W} \quad 04-16.65$ 0977 STENAA FUSEATA $14^{\circ} 59 \mathrm{M}-170^{\circ} 37 \mathrm{~W}$ 04-16-65 0978 Puffinus parificus $14{ }^{\circ} 59 \mathrm{~N}-170^{\circ} 37^{\mathrm{W}} 04-16.65$ 0979 STENA FUSCATA $14055 \mathrm{~N}-170^{\circ} 41 \mathrm{~W} \quad 04-16-65$ 0980 PhaEthon lepturus $14047 \mathrm{~N}-170^{\circ} 50^{\mathrm{W}} \mathrm{W} \quad 04-16-65$ 0981 STENNA FUSCATA $14036 \mathrm{~N}-171^{\circ} 03^{\circ} \mathrm{W} \quad 04-16-65$ $0982 " 14^{\circ} 366^{\prime} \mathrm{N}-171^{\circ} \mathrm{O}^{\prime} \mathrm{W} \quad 04-16-65$ 0983 " $\quad 14^{\circ} 36^{\prime} \mathrm{N}-171^{\circ} 03^{\prime} \mathrm{W} \quad 04-16-65$ 0984 " $\quad 14^{\circ} 36 \mathrm{~N}-171^{\circ} 03^{\prime} \mathrm{W} \quad 04-16-65$ $0985 " 14^{\circ} 36 \mathrm{~N}-171^{\circ} 3^{\prime} \mathrm{W} \quad 04-16-65$ $0986.14^{\circ} 36^{\prime} \mathrm{N}-171^{\circ} 0^{\circ} \mathrm{W} \quad 04-16.65$ 0987 Phanthon lepturus 13040'N-172 49i $04-17-65$ 0988 PhAEThON RUbrienuda $13^{\circ} 40^{\prime} \mathrm{N}-172^{\circ} 49^{\prime} \mathrm{W} \quad 04-17-65$ 0989 Sala dAcTylatra $14^{\circ} 14 \mathrm{~N}-172^{\circ} 43^{\prime} \mathrm{W} \quad 04-17.65$ 0990 Phathon rubricauda $1426 \mathrm{~N}-172^{\circ} 32^{\mathrm{W}} \mathrm{W} \quad 04-17-65$ 0991 OCEANOdVOMA IEucorhoA $14^{\circ} 32^{\circ}-172^{\circ} 24^{\mathrm{W}} \quad 04-17-65$ 0992 SulA DACTYLATHA $16^{\circ} 28^{\prime} \mathrm{N}-171^{\circ} 10^{\prime} \mathrm{W}$ $04-18 \cdot 65$ 0993 STEVNA FUSCATA $16^{\circ} 36 \mathrm{~N}-171^{\circ} 26^{\circ} \mathrm{W}$ $04-18-65$ 0994 $16^{\circ} 36 \mathrm{~N}-171^{\circ} 26 \mathrm{~W}$ $04-18 \cdot 65$ 0995 $16^{\circ} 36 \mathrm{~N}-171^{\circ} 26 \mathrm{~W}$ $04-18-65$ 
0996 Sula daEtylaTra $16^{\circ} 3111-17129 \mathrm{~W}$ 04-18-65 0997 OCEANOdVOMA IEUCOrhoA $15^{\circ} 58 \mathrm{MN}-172^{\circ} 04^{\prime} \mathrm{W}$ 04-18-65 0998 STENA FUSEATA $15^{\circ} 444^{\prime N}-172^{\circ} 20^{\prime} \mathrm{W} 04-18.65$ $0999 \quad " 15^{\circ} 44 \mathrm{~N}-172^{\circ} 20 \mathrm{~W} 04-18.65$ $1000 " 15^{\circ} 44 \mathrm{~N}-172^{\circ} 20^{\circ} \mathrm{W}$ 04-19-65 $1001 " 15^{\circ} 44 \mathrm{~N}-172^{\circ} 20^{\prime} \mathrm{W} \quad 04-18-65$ $1002 " 15^{\circ} 44^{\prime} \mathrm{N}-172^{\circ} 20^{\circ} \mathrm{W} \quad 04-18-65$ 1003 " $15^{\circ} 44 \mathrm{ill}-172^{\circ} 20^{\prime} \mathrm{W} \quad 04-18-65$ $1004 \quad 15^{\circ} 44 \mathrm{~N}-172^{\circ} 20^{\circ} \mathrm{W} \quad 04-18 \cdot 65$ $1005.15^{\circ} 44 \mathrm{MN}-172^{\circ} 20 \mathrm{~W} \quad 04-18-65$ 1006 " $\quad 15^{\circ} 44 \mathrm{iN}-172^{\circ} 20 \mathrm{~W} \quad 04-18-65$ 1007 Pierodromat hypoleuca 15044 $\mathrm{Ml}-172^{\circ} 20^{\prime} \mathrm{W} \quad 04-18.65$

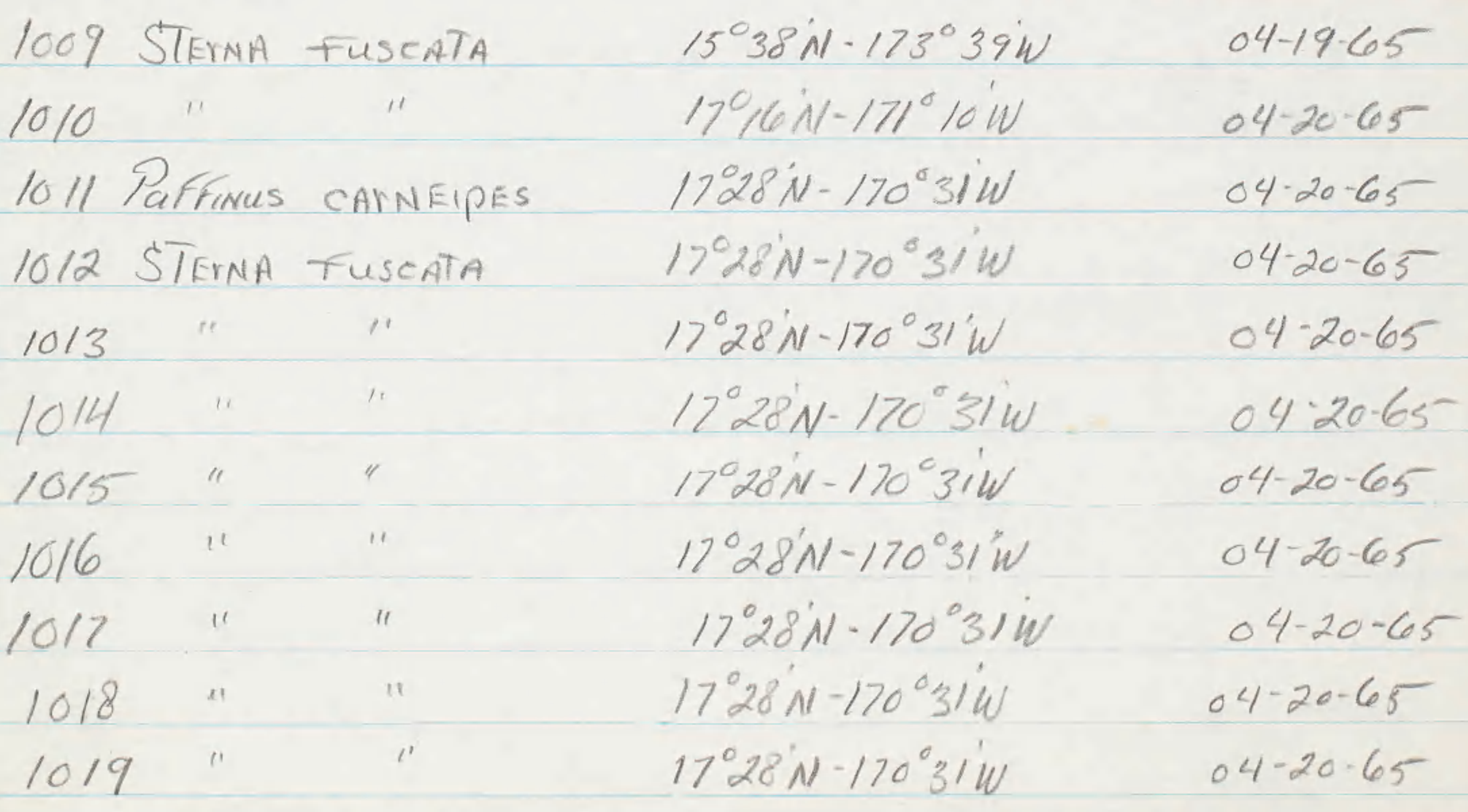


1020 STENAA FUSCATA $17^{\circ} 28 \mathrm{Ml}-170^{\circ} 31 \mathrm{~W}$ 04-20-65

1021

$17^{\circ} 28^{\circ} \mathrm{N}-170^{\circ} \mathrm{3iW} \quad 04-20-65$

1022

$17^{\circ} 28^{\prime} \mathrm{N}-170^{\circ} \mathrm{3i} \mathrm{W}$

$04-20-65$

1023

$17^{\circ} 28 \mathrm{Al}-170^{\circ} 3 \mathrm{iW}$

$04-20-65$

1024

$17^{\circ} 28^{\circ} \mathrm{N}-170^{\circ} 31 \mathrm{~W}$

$04-20-65$

1025

$17^{\circ} 28 \mathrm{~N} \cdot 170^{\circ} 3 \mathrm{iW} \quad 04-20-65$

1026 Puffinas parificas $17040^{\prime} \mathrm{N}-169^{\circ} 57 \mathrm{~W}$ 04-20-65

1027 Paffinus pacificus $17^{\circ} 40^{\prime} \mathrm{N}=16957 \mathrm{~W}$ 04-20-65

1028 STERA FUSCATA $17^{\circ} 422^{\mathrm{N}}-169^{\circ} 47^{\prime} \mathrm{W}$ 04-20-65

1029 SulA dactylnTra $1838 \mathrm{~N}-166^{\circ} 51$ 'W $0421-65$

1030 STENA FUSEATA $18^{\circ} 48^{\prime} \mathrm{N}-166 \% \mathrm{~W} \quad 04-21-65$

1031 STERAA FUSEATA 18048 A1-166\% $19 \mathrm{~W}$ 04-21-65

1032 Puffinas pacificus 18\%81-166\%19iW 04-21.45

1033 STERAA FUSCATA $19^{\circ} 02^{\circ} \mathrm{N}-165^{\circ} 36^{\circ} \mathrm{W}$ 04-21-65

1034 Paffinus parificus $19^{\circ} 06^{\prime} \mathrm{N}-165^{\circ} 23^{\prime} \mathrm{W}$ 04-21-65

1035 FrEgATA Minor $19^{\circ} 06^{\prime} N 1-165^{\circ} 23^{\prime} \mathrm{W} \quad 04-21-65$

1036 Puffinas pacificus $19^{\circ} 44 \mathrm{~h}-163 \% 13 \mathrm{~W} \quad 04-22.65$

1037 Sula dactylatra 19045 N-163\% 04.22 .65

1038 STERA FUSEATA $19^{\circ} 48^{\prime} \mathrm{N}-163^{\circ} 02^{\prime} \mathrm{W} \quad 04-22.65$

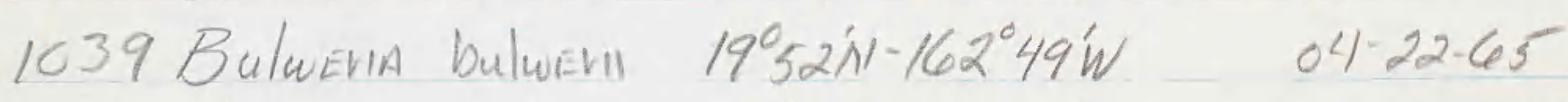

1040 BulwerA bulwenl $19^{\circ} 52^{\prime} \mathrm{N}-162^{\circ} 17 \mathrm{~W} \quad 04.22 .65$

1041 STERAA FUSCATA $19^{\circ} 52^{\prime} N 1-162^{\circ} 47^{\prime} \mathrm{W} \quad 04-22.65$

1042 STERA FUSEATA $195^{\circ} 2^{\prime N}-162^{\circ} 47 \mathrm{~W} 04.22 .65$ 
1043 STERNA FUSENTA $19^{\circ} 52^{\prime} \mathrm{NI}-162^{\circ} 47^{\prime} \mathrm{W} 04.22 .65$ 1044 STENMA FUSEATA $19^{\circ} 55^{\mathrm{N}}-162^{\circ} 40^{\prime} \mathrm{W}$ 04.22.65 1045 Bulweria bulwern $20^{\circ} 00 \mathrm{M1}-162^{\circ} 19 \mathrm{~W}$ 04.22.65 1046 BulwenA bulwern $20^{\circ} 02^{\prime} \mathrm{N}-162^{\circ} 9^{\circ} \mathrm{W}$ 04-22.65 1047 Bulwenta bulweml $20^{\circ} 03 \mathrm{~N}-162^{\circ}$ o5 W $04.22-65$ 1048 Bulweria bulwern $20^{\circ} 86 \mathrm{~N}-161^{\circ} 52 \mathrm{~W}$ 04-22-65 1049 Bulueria bulwenl $20^{\circ} 88^{\mathrm{N}} \mathrm{N}-161^{\circ} 47^{\mathrm{W}} \mathrm{W} \quad 04-22-65$ 1050 SulA sula $20^{\circ} 13^{\prime} \mathrm{N}-161^{\circ} 20^{\circ} \mathrm{W} \quad 04-22.65$

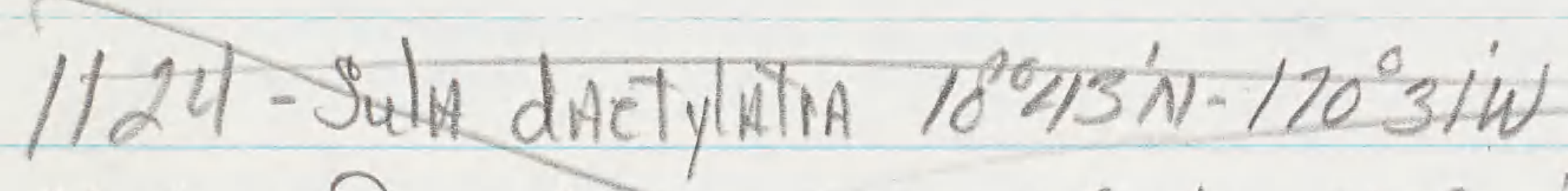
$05 \cdot 21-65$ 1151 - Diomedina NTIGLEES 2120 N. $167^{\circ} 41 \mathrm{~W}$ 05.22 .65

1152 - DiomfdiA XIIGMpes 2 $05-22-65$

1214

1215

1232

$1236-$

1241

1242 -

$12 \sqrt{72-}$ 


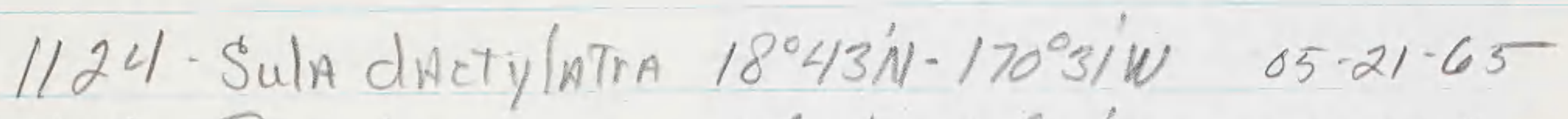
1151 - DiOMEdEA NIgRTPES $21^{\circ} 20^{\prime} \mathrm{N}-167^{\circ} 41_{\mathrm{W}} \mathrm{W}-22.65$

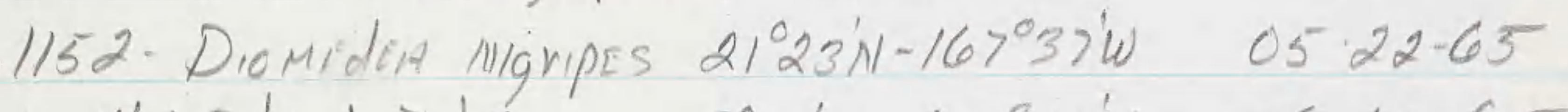
1214 - SulA dAElylatrA $07^{\circ} 02^{\prime} \mathrm{Al}-152^{\circ} 27 \mathrm{~W} \quad 06-10-65$ 1215 - SulA dAETYIATAA $07^{\circ} 02 \mathrm{~N}-152^{\circ} 28^{\prime} \mathrm{W} \quad 06-10-65$ 1232 . SulA dadlylatia $06^{\circ} 20^{\prime} \mathrm{N}-153^{\circ} 14 \mathrm{~W}, 06-10.65$ 1236 - SulA dactylatra $06^{\circ} / 3 \mathrm{M}-153^{\circ} 34^{\prime} \mathrm{W} 06-10-65$ 1241 -STERNA TUSEATA $06^{\circ} 11 \mathrm{N1}-155^{\circ} 20^{\circ} \mathrm{W} \quad 06-11.65$ 1242 -Sula dadylnTra $06 \% 111-155^{\circ} 21 \mathrm{~W} \quad 06-11-65$ 1247 - Sula dactylatra $06^{\circ} 21 \mathrm{~N}-156^{\circ} 07 \mathrm{~W} \quad 06-11-65$ 1256 - FrEqATA MiNor $07^{\circ} 05 \mathrm{~N}-158044 \mathrm{~W} \quad 06-12.65$

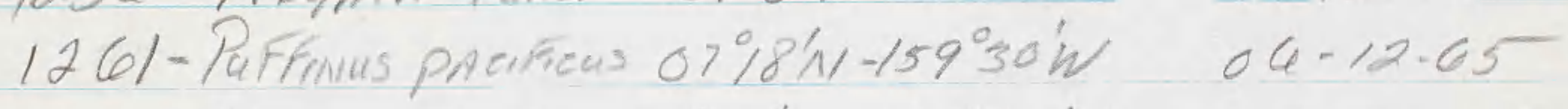
1299 - STERAIA FUSEATA $19^{\circ} 40^{\prime} \mathrm{N}-163^{\circ} 45^{\mathrm{W}} \quad 06-23-65$ 1300 - " $19 \% 0^{\circ} \mathrm{N}-163045 \mathrm{~W} \quad 06-23.45$ 1302 - "19\%91-16309W 06.23-65 1306 - Paffinus panticas $19^{\circ} 50 \mathrm{~N} \cdot 163^{\circ} 0^{4} \mathrm{lll}$ 06-23-05 1308 - Ruffinas prociñus $19^{\circ} 50^{\prime} \mathrm{N}-163^{\circ} 0^{4} \mathrm{~W}$ 06.23-65 1314 - FEEATA MEXIOV $20^{\circ} 28^{\circ} \mathrm{N}-160^{\circ} 49 \mathrm{~W}$ $06 \cdot 24 \cdot 65$

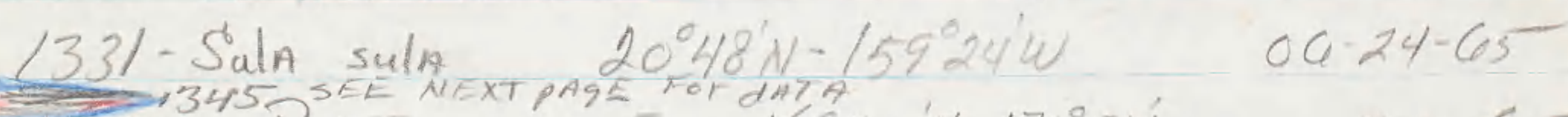

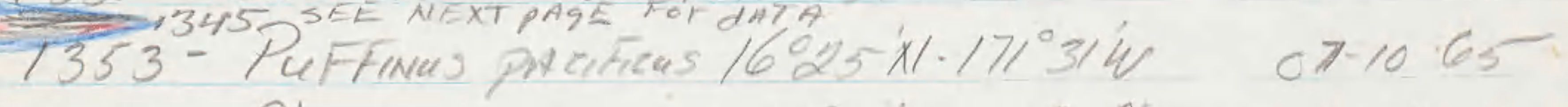
1357 - PhinEThoN rubucpudn $14^{\circ} 38 \mathrm{~N}-173^{\circ} 48 \mathrm{~W}$ 0 $7-11-65$ 1358 - STERNA TUSERA $16^{\circ} \mathrm{C} 4 \mathrm{M} 1-174 \% \mathrm{~W}$ 07-11-65

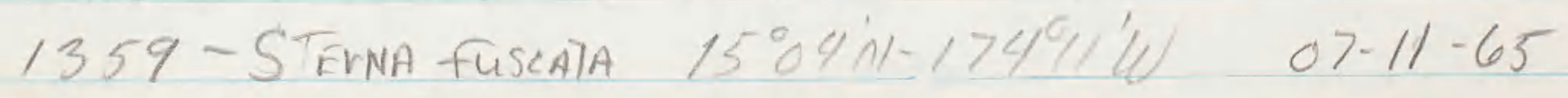




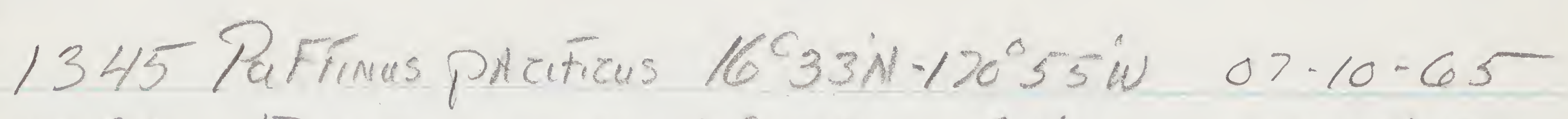

1360 . STEIMA FUSCATA $15^{\circ} 04 \mathrm{~N}-174^{\circ} 11 \mathrm{~W}$ 0?-11.65

1361 - STERMA FUSEATA $15^{\circ} 0411-174^{\circ} 11 \mathrm{~W}$ 07-11-65 


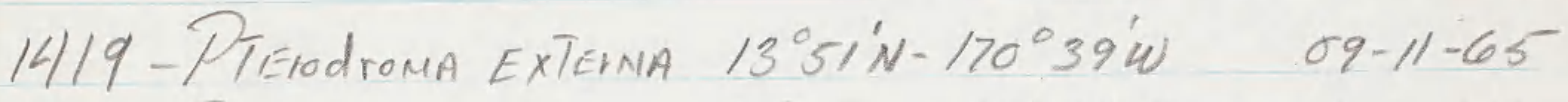

$1420^{-}$PTETodroma EXTEMNA $13^{\circ} 30^{\prime} \mathrm{N1}-170^{\circ} 48^{\prime} \mathrm{W}$

$09-11-65$

1421 - PTErodroma ExTEMAa 10\% $12 \mathrm{~N}-172^{\circ} 14^{\prime} \mathrm{W}$

$09-12-65$

1422 - SulA daCTylatra $09^{\circ} 10 \mathrm{~N}-172^{\circ} 44 \mathrm{~W}$

$09-12 \cdot 65$

1423 - STENA FUSEATA $09^{\circ} 0^{\circ} \mathrm{N}-172^{\circ} 49^{\prime} \mathrm{W}$

$69-12-65$

1425 - PTitodroma exterma $6022 \times 1-173^{\circ} 48 \mathrm{~W}$ 09-13-65

1426 - Paffinas pacificas $05^{\circ} 45 \mathrm{~N}-174^{\circ} 04^{\prime} \mathrm{W} 09-13-65$

1427 - Paffinus parificas $05^{\circ} 45^{\prime} \mathrm{N}-174^{\circ} 04 \mathrm{~W}$ 09-13.65

1428 -Paffinas greisas $05^{\circ} 45^{\mathrm{N}}-174^{\circ} 0^{\prime \prime} \mathrm{W} \quad 09-13-65$

1430 - Puffinas tenarostro $05^{\circ} 14$ 'N-176 $06 \mathrm{~W}$

$09-14-65$

1431 - PTerodroma EXTENAa $05^{\circ} 14 \mathrm{~N}-176^{\circ} 09^{\prime} \mathrm{W}$

$09-14 \cdot 65$

1432 - Paffinus paciricas $05^{\circ} 15 \mathrm{M}-176^{\circ} 17 \mathrm{~W}$

$09-14-65$

1433 - Puffinus Tenuitostris 05\% $16 \mathrm{~N}$ - $176^{\circ} 24^{\mathrm{W}}$

$09-14 \cdot 65$

1434- Paftinus TEnuirostris 05 $177^{\prime} \mathrm{N}-176^{\circ} 26^{\prime} \mathrm{W}$

$09-14 \cdot 65$

1435-STENA FUSEATA $02^{\circ} 40^{\prime} \mathrm{N}-176^{\circ} 27^{\prime} \mathrm{W}$

$09-15-65$

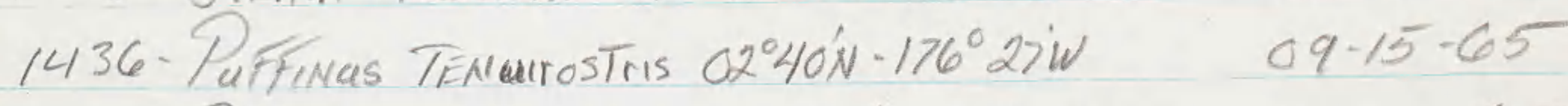

1437-PuFfinas TEnuirostris $02^{\circ} 33^{\prime} \mathrm{N}$ - $176^{\circ} 25 \mathrm{~W} \quad 09-15.65$

1438-Puffinas pacericas 02028' $-176^{\circ} 24$ W 09-15-65

1439 -STERNA FUSCATA $02^{\circ} 03 \mathrm{~N}-176^{\circ} 24^{\prime} \mathrm{W} \quad 09-15-65$

1440 - Puffinas pacificas $01{ }^{\circ} 37 \mathrm{~N}-176^{\circ} 25^{\mathrm{W}} \quad 09-15-65$

1441 - Puffinas Tenairostris $00^{\circ} 0^{\prime} 5-126 \% 3 \mathrm{~W}$ 09-16.65 
1442 Paffrnas tenuirostris $00^{\circ} 06^{\prime} S-176^{\circ} 44 \mathrm{~W}$ 1443 Puuinlis dominica $80^{\circ} 11 s^{\prime}-176^{\circ} 49$ W 1444 STERMA FUSEATA $00^{\circ} 23^{\prime} \mathrm{s}-176^{\circ} 58^{\circ} \mathrm{W}$ 1445 Anous stolidus $01019 \mathrm{N1}-177^{\circ} 32^{\mathrm{W}} \mathrm{W}$ 1446 Anous stolidus $01 \% 19^{\prime} \mathrm{N}-177^{\circ} 32 \mathrm{~W}$

1448 Sula dactylatra o/ $16^{\prime} \mathrm{N}-176^{\circ} 44^{\prime} \mathrm{W}$ 1449 STENAA FUSEMTA $01 \% 15 \mathrm{~N}-176 \%$ 1450 Sula daetylatra $00^{\circ} 15^{\prime} S^{\prime}-175^{\circ} 56^{\prime} \mathrm{W}$ 1451 Anlous stolidus $00^{\circ} / 55-175^{\circ} 56^{\circ} \mathrm{W}$ 1452 STEINA FUSCATA $00^{\circ} 32^{\prime} 5^{\circ} 175^{\circ} 52 \mathrm{~W}$ 1453 ARENARIA IMTERPEES $01026^{\prime} 5-176^{\circ} 28^{\prime} \mathrm{W}$ 1454 STERNA FUSCATA $00^{\circ} / 5 \mathrm{~N}-177^{\circ} 33^{\prime} \mathrm{W}$ 1455 STERAA FUSCATA $00^{\circ} / 5^{\prime} \mathrm{N}-177^{\circ} 33^{\mathrm{W}}$ 1456 Paffinas pacificus $00^{\circ} 18^{\prime} \mathrm{N}-177^{\circ} 29^{\mathrm{W}}$ 1457 STERMA FUSCATA $00^{\circ} 25^{\prime} \mathrm{N}-172^{\circ} 15^{\prime} \mathrm{W}$ 1458 Puffinus pacificus $00^{\circ} 40^{\prime} \mathrm{N1}-176^{\circ} 50^{\circ} \mathrm{W}$ 1459 Sula sula $03^{\circ} 48^{\prime} S-174^{\circ} 56^{\prime} \mathrm{W}$ 1460 Aremaria interpres $03^{\circ} 48^{\circ} 5^{\circ}-174^{\circ} 59 \mathrm{~W}$ 1461 Sula sula $13^{\circ} 435^{\circ}-170^{\circ} 38^{\circ} \mathrm{W}$ 1462 PluUialis do MiNica $13^{\circ} 45^{\prime}-170^{\circ} 37^{\prime} \mathrm{W}$ 1463 STERNA FUSEATA $06^{\circ} 03^{\prime} S-173^{\circ} 37 \mathrm{~W}$ 1464 STERNA FUSEATA $06^{\circ} 03^{\prime} \mathrm{S} \cdot 173^{\circ} 37^{\prime} \mathrm{W}$
$09-16-65$ $09-16-65$ $09 \cdot 16 \cdot 65$ $09-17-65$ $09-17-65$ $09-17-65$ $09 \cdot 17-65$ $09-18-65$ $09-18.65$ $09-18 \cdot 65$ $09-18-65$ $09-19-65$ $09-19.65$ $09-19 \cdot 65$ $09-19 \cdot 65$ $09-19-65$ $10-14-65$ $10-14-65$ $10-06.65$ $10-06-65$ $10-13-65$ $10-13-65$ 
1465 STERA FUSCATA $06^{\circ} 03^{\prime} 5-173^{\circ} 45^{\mathrm{W}}$ $10-13-65$ 1466 $06^{\circ} 03^{\prime} 5-173^{\circ} 45 \mathrm{~W}$ $10-13 \cdot 65$

1467 $06^{\circ} 0^{\circ} \mathrm{s}-173^{\circ} 45 \mathrm{~W}$ $10-13-65$ 1468 HeTEROSEELUS INCANUM $03^{\circ} 56^{\prime} \mathrm{S}-173^{\circ} 48^{\prime} \mathrm{W}$ $10-13-65$ 1469 Anous sTolidus $00^{\circ} 13^{\prime} \mathrm{S}-176^{\circ} 09^{\mathrm{W}} \mathrm{W}$ $10-15 \cdot 65$ 1470 Puffinus TEnairostris $00^{\circ} 10^{\prime} \mathrm{N}-176^{\circ} 26 \mathrm{~W} \quad 10-15 \cdot 65$ 1471 Pluvialis dOMINica 0102711-176\% W 10.16-65

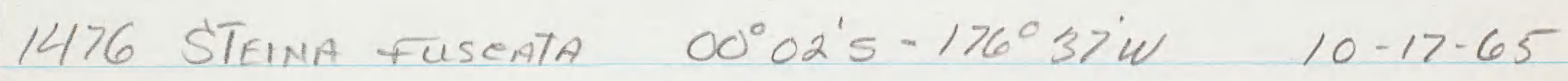
1478 . STEMMA FUSCATA $00^{\circ} 06^{\circ} \mathrm{N}-176^{\circ} 50^{\circ} \quad 10-1765$ 1482. PTEROdTOMA INEXPECTATA $00^{\circ} 20^{\prime} \mathrm{N}-176^{\circ} 52^{\prime} \mathrm{W} \quad 10-17.65$

1485 OEFANOdVOMA CASTrO $02^{\circ} 00^{\prime} \mathrm{N}-177^{\circ} 30^{\prime} \mathrm{W} \quad 10-18-65$

1487 PhaEThon lepturus $01^{\circ} 39^{\mathrm{N}}-178^{\circ} 0^{\circ} \mathrm{W} \quad 10-18-65$ 1488 - Plunales dominica $00^{\circ} 15 \mathrm{~N}-177^{\circ} 38 \mathrm{~W} \quad 10-19-65$ 1489 - DCEAMTES OCEAMICUS $00^{\circ} 20^{\circ} \mathrm{N}-177^{\circ} 20^{\circ} \mathrm{W} \quad 10-19.65$ 1490 OCEANites Oceanicus $00^{\circ} 20^{\prime} \mathrm{N}-177^{\circ} 28^{\prime} \mathrm{W} \quad 10-19-65$ 1491 OCEAMITES OREANICUS $00^{\circ} 20^{\circ} \mathrm{N}-177^{\circ} 20^{\circ} \mathrm{W} \quad 10-19-65$ 1494 Gygis alba $00^{\circ} 26^{\prime} \mathrm{S}-177^{\circ} 38^{\prime} \mathrm{W} \quad 10-19-65$ 
1502 PTErodroma IN EXPEETATA 00 $26^{\prime} \mathrm{S}^{\circ}-177^{\circ} 37^{\prime} \mathrm{W} \quad 10-20.65$

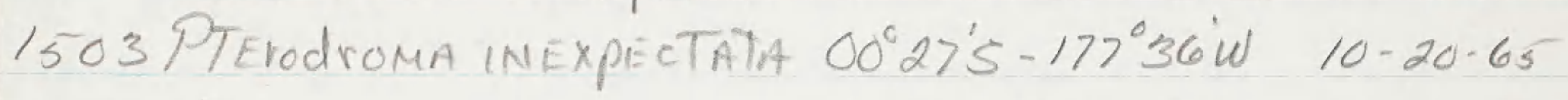
15,10 STEMAA FUSEATAA $00^{\circ} 27 ' 5-177^{\circ} 36 \mathrm{~W} \quad 10-20-65$ 1515 OCEANITES oceANICUS $00^{\circ} 27 \mathrm{~S}-177^{\circ} 36^{\prime} \mathrm{W} \quad 10-20-65$ 1519 STEMAA FUSEATA $00^{\circ} 34^{\prime} \mathrm{N}-176^{\circ} 20^{\circ} \mathrm{W} 10.22-65$ 1521 STERAA FUSCATA $00^{\circ} 14^{\prime} \mathrm{N}-175^{\circ} 52^{\prime} \mathrm{W} \quad 10.22-65$ 1527 PhaEThoN lepturus $01^{\circ} 29^{\prime}$ 's-1730 $08^{\circ} \mathrm{W} \quad 10-23.65$ 1531 STERMA PARAdISAEA $011^{\circ} 52^{\prime} \mathrm{S}-172^{\circ} 53^{\prime} \mathrm{W} \quad 10-23-65$ 1533 Sula Sula $02^{\circ} 22^{\prime} \mathrm{s}-170^{\circ} 43^{\prime} \mathrm{W} \quad 10.24-65$ 1542 Sula sula $00^{\circ} 17^{\prime} 5-167^{\circ} 42^{\prime} \mathrm{W} \quad 10.25-65$ 1547 SulA dACTYIATrA O007's-164 $53^{\circ} \mathrm{W} \quad 10-26-65$ 1548 SulA daETyIATrA $00^{\circ} 07^{\prime} S-164^{\circ} 53^{\prime} \mathrm{W} \quad 10-26-65$ 1551 Gyges AlbA $00^{\circ} 03^{\circ}-164^{\circ} 53 \mathrm{~W} \quad 10-26-65$ 1552 OCEANOdVOMA LEUCOThOA $00^{\circ} 0^{\prime} \mathrm{N}-164^{\circ} 14^{\mathrm{W}} \mathrm{W} \quad 10-26-65$ 1567 OCEANOdrOMA IEUCOrhOA $06^{\circ} 41^{\prime} \mathrm{N}-157^{\circ} 29^{\circ} \mathrm{W} \quad 11-01-65$ 1570 PTEROdrOMA AlbA $06^{\circ} 53^{\prime} \mathrm{N}-157^{\circ} 27^{\circ} \mathrm{W} \quad 11-01-65$ 1571 PTEROdROMA EXTERNA $07^{\circ} 05^{\prime} \mathrm{N}-157^{\circ} 28^{\circ} \mathrm{W} \quad 11-01-65$ 1572 PTERodroma hypoleucA $07^{\circ} 11 \mathrm{NN}-157^{\circ} 27^{\mathrm{W}} \quad 11-01-65$ 1573 PTErodroma EXTERNA $14^{\circ} 08^{\prime} \mathrm{N}-157^{\circ} 39^{\circ} \mathrm{W} \quad 11-03.65$ 1574 PTErodroma hypdicuch $14^{\circ} 11 \mathrm{~N}-157^{\circ} 38^{\prime} \mathrm{W} \quad 11-03-65$ 1575 PTERodroma hypoleuca $18^{\circ} 46^{\prime \prime N}-157^{\circ} 48^{\circ} \mathrm{W} \quad 11.04-65$ 1576 OCEANOdIOMA IEUCOrhOA $18^{\circ} 55^{\prime} \mathrm{N}-157^{\circ} 48^{\circ} \mathrm{W} \quad 11-04-65$ 
5592 Anous stolidus CANTON ATOll, PhOENXI Islands,o6-01-65

5756 Sula dactylatra Howland Island, Pacific Ocenan 09-16.65 5758 Anous minutus

5760 Sula sula MCKEAN TS., PhoENix Tslands $09-16-65$

5764 Phalethon rubricauda

$09-22 \cdot 65$

5765 FrEgATA ARIEI

$09 \cdot 23 \cdot 65$

5766 SulA dAETy/ATrA

$09 \cdot 23 \cdot 45$

5767 Sula dactylatra

$09-23-65$

5768 PuFrinus paciñcus Hull Is,

$09-23.65$

5769 Heteroschelas incanum Phoenix.Is.,

$09-24 \cdot 65$

5770 Erolia MElANOTOS PhOENIX IS.,

$09-30-65$

5721 Sula leurogaster Phomexis,

$09 \cdot 30-65$

$09-30 \cdot 65$

5806 Sula dartylatra Phocnix Is, Phomexix Islands 09-30-65 5807 Erolia Melanotos Enderbury Is., "10-02.65 5808 FrOLIA MElANOTOS $10 \cdot 02 \cdot 65$

5809 SulA SUlA

$10-02-65$

5811 STEIna fusenta Mull Is, PhoEnix Islands 09-24-65 5812 STEMNA FUSCATA

$09 \cdot 24 \cdot 65$

5814 Erolia ACUminata Baker Is, PACific Ocean 09-18.65

5815 Numenius Tahitiensis

$09-19 \cdot 65$ 
5816 Heteroscelus incanum Baker Is. PAcific ocean 09-16-65 5818 LIMOSA IAPPONICA $10-19-65$ 5819 Frolina ACUMINATA $10-20 \cdot 65$ 5820 VINI Kuhl, ChNistmas Is. /INE Islands 10-28-65 5821 ThalASSEUS bergll 10.28 .65 5822 VINI Kuhll $10-28-65$

5823 Gygis Alba $10-28.65$

5824 ThaldssEas bergll $10-28 \cdot 65$

5825 Thalasseus berg" $10-28 \cdot 65$

5826 PluUinlis dominica $10-28 \cdot 65$

5827 Plavials dominica $10-28-65$

5828 NAMENIUS TAhITIENSIS $10 \cdot 28 \cdot 65$ 5829 Nlamenias tahitIENSIS $10 \cdot 28 \cdot 65$

5831 Sula daETyIATrA $10-29-65$ 5833 SPATULA ElYPEATA $10-29-65$

5863 PTEROdTOMA AlbA $10-30-65$ 5864 PTEROdROMA AlbA $10 \cdot 30-65$ 5865 PTEROdTOMA AlbA $10 \cdot 30-65$

5867 SulA suln $10-30-65$ 


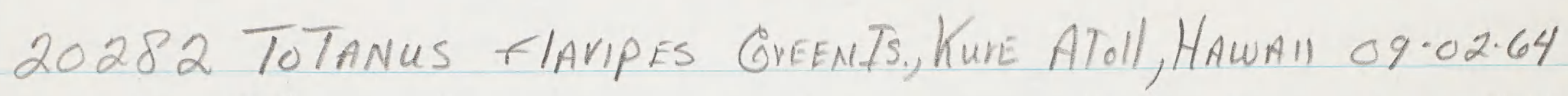
20289 SulA SulA $09-11-64$ 20440 Gygis Alba $02-25-65$

20477 PhAEThON rubricauda $05 \cdot 25 \cdot 65$

20478 SulA SulA $05 \cdot 27-65$

20481 FrEgATA MINOR $06-03-65$

20482 FrEgATA MiNOr $06-03-65$

20485 PhaEThon rubricaudA

$07-01-65$

30031 Arenaria inTerpres Bikar AToll, Marshall Islands 1015-64 30033 ArENAMA INTEIPRES $10-15-64$

15008 Mimus polyglotTos French FrigAte Shonls, HAwAll08.18-65 
Field

Tumber Species

0951 Sterna ruscata

0952 Sula dactylatra

0953 Puffinus pactricus

0954

0955 Sula dactylatra

0956

0957

0958

0959

0961

0962

0963 Sterna puscata

0964. Phaethon rubricauda

0965 Sterne Puscata

0966 Puffinus griesus

0967 Sula sula

0960

0969 " dectylatra

0970 Sterns Rucate

0971

0972

0973

0974

0975
Date

Collected

Ioce Isty

$04-05-65$

$04-06-65$

$04-16-65$

$04-06-65$

oh. $06-65$

$04-06-65$

$04-17-65$

$04-17-65$

$04-07-65$

$04-07-65$

$04-07-65$

$04-11-65$

$04-11-65$

$04-13-65$

$04-13-65$

$04-14-65$

$04-14-65$

$04-16-65$

$04-16-65$

$04-16-65$

$04-16-65$

$04-16-65$

$04-16-65$

$04-16.65$ $11^{\circ} 31^{\prime} 11-150^{\circ} 31^{\prime} \mathrm{W}$

$09^{\circ} 36 \mathrm{H}-151$ \%

$09^{\circ} 27^{\prime N}-151^{0147} \mathrm{~W}$

$09^{0} 27^{1} \mathrm{~m}^{-151^{\circ} 47^{\prime} \mathrm{W}}$ $08^{\circ} 56^{\prime} \mathrm{N}-152^{\circ} 17^{\prime} \mathrm{W}$

$08^{\circ} 4^{\prime} T^{\prime} \mathrm{N}-1522^{\circ} 27^{\prime} \mathrm{W}$

$08^{\circ} 07^{\prime} \mathrm{H}-153^{\circ} 37^{\prime} \mathrm{H}$

$08^{\circ} 07^{\prime} \mathrm{N}-155^{\circ} 37^{\circ} \mathrm{W}$

$08^{\circ} 09^{\prime} \mathrm{m}-153^{\circ} 45^{\prime} \mathrm{W}$

$03^{\circ} 28 \cdot N-153^{\circ} 35 \mathrm{~W}$

$08^{\circ} 28 \mathrm{~N}-153^{\circ} 35^{\prime} \mathrm{W}$

$16^{\circ} 07^{\prime} \mathrm{I}-161^{0} 34^{\prime} \mathrm{W}$

$16^{\circ} 08^{\prime} \mathrm{N}-161^{\circ} 50^{\prime} \mathrm{W}$

$16^{\circ} 37^{\prime} \mathrm{N}-169^{\circ} \mathrm{O} \cdot \mathrm{W}$

$15^{\circ} 50^{\prime} \mathrm{N}-169^{\circ} 16^{\prime} \mathrm{W}$

$13^{\circ} 30^{\prime} 1-169^{\circ} 38 \cdot \mathrm{W}$

$13^{0} 14 \cdot \mathrm{w}-169^{\circ} 58 \mathrm{w}$

$15^{\circ} 24 \cdot 169^{\circ} 54 \mathrm{~W}$

$15^{\circ} 30^{\prime} \mathrm{N}-170^{\circ} 03^{\prime} \mathrm{W}$

$15^{\circ} 27^{\prime} \mathrm{N}-170^{\circ} 12^{\prime} \mathrm{W}$

$15^{\circ} 24 \cdot N \cdot 170^{\circ} 16^{\prime}$

$15^{\circ} 22^{\prime} \mathrm{N}-170^{\circ} 19^{\prime} \mathrm{W}$

$15^{\circ} 10^{\prime} \mathrm{N}-170^{\circ} 28 \cdot \mathrm{W}$

$35^{\circ} 10^{\prime} \mathrm{M}-170^{\circ} 28^{\prime} \mathrm{w}$ 


\begin{tabular}{|c|c|c|c|}
\hline 0976 & Sula sula & $04-16-65$ & $15^{\circ} 10^{\prime} \mathrm{N}-170^{\circ} 31^{\prime} \mathrm{W}$ \\
\hline 0977 & Sterna fuscata & $04-16-65$ & $14^{\circ} 59^{\prime} \mathrm{W}-170^{\circ} 37^{\prime} \mathrm{W}$ \\
\hline 0978 & Puffinus pacipicus & $04-16-65$ & $14^{\circ} 59^{\prime} \mathrm{NH}-170^{\circ} 37^{\prime} \mathrm{W}$ \\
\hline 0979 & Sterna fuscata & $04-16-65$ & $14^{\circ} 55^{\prime} \mathrm{N}-170^{\circ} 41^{\prime} \mathrm{W}$ \\
\hline 0980 & Phaethon Lepturus & $04-16-65$ & $24^{\circ} 47^{\prime} \mathrm{NN}-170^{\circ} 50^{\prime} \mathrm{W}$ \\
\hline 0981 & Sterna fuscata & $04-16-65$ & $14^{\circ} 36^{\prime} \mathrm{W}-172^{\circ} 03^{\prime} \mathrm{W}$ \\
\hline 0932 & " $\quad$ " & $04-16-65$ & $14^{\circ} 36^{\prime} \mathrm{W}-171^{\circ}$ O3' W \\
\hline 0983 & " $\quad$ " & $04-16-65$ & $14^{\circ} 36^{1} \mathrm{~N}-171^{\circ} 03^{1} \mathrm{~W}$ \\
\hline 0984 & $x^{n}+\infty=n$ & $04-16-65$ & $14^{\circ} 36^{\prime} \mathrm{Wl}-171^{\circ} 03^{\prime} \mathrm{W}$ \\
\hline 0985 & $" n=1$ & $04-16-65$ & $14^{\circ} 36^{\prime} \mathrm{W}-171^{\circ} 03^{\circ} \mathrm{W}$ \\
\hline 0986 & $n$ & $04-16-65$ & $14^{\circ} 35^{\prime} \mathrm{N}-171^{\circ} 03^{\prime} \mathrm{W}$ \\
\hline 0987 & Phaethon lepturus & $04-17-65$ & $13^{\circ} 40^{\prime} \mathrm{N}-172^{\circ} 49^{\prime} \mathrm{W}$ \\
\hline 0988 & " rubriceuda & $04-17-65$ & $13^{\circ} 40^{\prime} \mathrm{N}-172^{\circ} 49^{\prime} \mathrm{W}$ \\
\hline 9089 & Sula dactylatra & $04-17-65$ & $14^{0} 14 \cdot 17-172^{0} 43^{7} \mathrm{~W}$ \\
\hline 0990 & Phaethon rubsicauda & $04-17-65$ & $14,26^{\prime} \mathrm{N}-172^{\circ} 32^{\prime} \mathrm{W}$ \\
\hline 0991 & Dceanodroma leucorhoa & $04-17-65$ & $14^{\circ} 32^{\prime} \mathrm{N}-172^{\circ} 24^{\prime} \mathrm{W}$ \\
\hline 0992 & Sula dactylatra & $04-18-65$ & $16^{\circ} 28^{\prime} \mathrm{NN}-171^{\circ} 10^{\prime} \mathrm{W}$ \\
\hline 0993 & Sterna fuscata & $04-18-65$ & $16^{\circ} 36^{\prime} \mathrm{N}-171^{\circ} 26^{\prime} \mathrm{W}$ \\
\hline 0994 & $n$ & $04-18-65$ & $16^{\circ} 36^{\prime} \mathrm{N}-171^{\circ} 26^{\prime} \mathrm{W}$ \\
\hline 0995 & " & $04-18-65$ & $16^{\circ} 36^{\prime} \mathrm{N}-172^{\circ} 26^{\prime} \mathrm{W}$ \\
\hline 0996 & $"$ " & $04-18-65$ & $16^{\circ} 31^{\prime} \mathrm{N}-172^{\circ} 29^{\prime} \mathrm{W}$ \\
\hline 0997 & Oceanodrona leucorhoa & $04-18-65$ & $15^{\circ} 58^{\prime} \mathrm{N}-172^{\circ} \mathrm{O} 4^{\prime} \mathrm{W}$ \\
\hline 0998 & Sterna Puscata & $04-18-65$ & $15^{\circ} 4, \cdot N-172^{\circ} 20^{\prime} w$ \\
\hline 0999 & $"$ & $04-18-65$ & $15^{\circ} 44^{\prime} \mathrm{N}-172^{\circ} 20^{\prime} \mathrm{W}$ \\
\hline 1000 & " & $04-19-65$ & $15^{\circ} 44^{\prime} \mathrm{N}-172^{\circ} 20^{\prime} \mathrm{W}$ \\
\hline 2001 & $n$ & $04-18-65$ & $1.5^{\circ} 44^{\prime} \mathrm{NN}-172^{\circ} 20^{\prime} \mathrm{W}$ \\
\hline & " & $04-18-65$ & $15^{\circ} 44 \cdot 1 \mathrm{~N}-172^{\circ} 20^{\prime} \mathrm{W}$ \\
\hline & $n$ & $04-18-65$ & $15^{\circ} 44^{\prime} \mathrm{N}-172^{\circ} 20^{\prime} \mathrm{W}$ \\
\hline
\end{tabular}




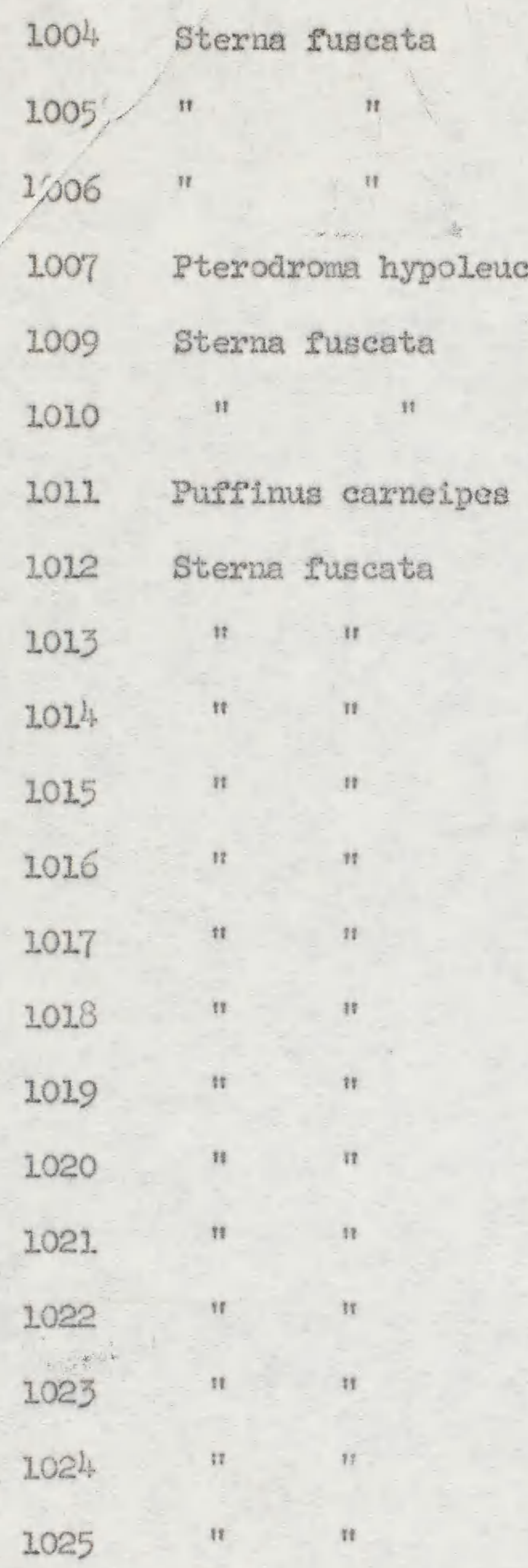

1026 Puffinus pacificus

1027

1028 Sterna fuscata

1029 Sula dactylatra

1030 Sterna Puscata

1031 " "

1032 Puffinus pacificus
$04-18-65$

$04-28-65$

$04-18-65$

$04-18-65$

$04-19-65$

$34-20-65$

$04-20-65$

$04-2 c-5$

$04-20-65$

$04-20-65$

$04-20-65$

$04-20-65$

$04-20-65$

$04-20-65$

$04-20-65$

$04-20-65$

$04-20-65$

$04-20-65$

$04-20-65$

$04-20-65$

$04-20-65$

$04-20-65$

$04-20-65$

$04-20-65$

$04-21-65$

$04-21-65$

$04-21-65$

$04-21-65$ $15^{\circ} 44^{\prime} \mathrm{N}-172^{\circ} 20^{\circ} \mathrm{W}$ $15^{0} 44^{\prime} \mathrm{N}-172^{\circ} 20^{\prime} \mathrm{W}$ $15^{\circ} 44^{\prime} \mathrm{NI}-172^{\circ} 20^{\prime} \mathrm{W}$ $15^{\circ} 44^{\prime} \mathrm{H}-172^{\circ} 20^{\prime} \mathrm{W}$ $15^{\circ} 38^{\prime} \mathrm{W}-173^{\circ} 39^{\prime} \mathrm{W}$ $17^{\circ} 16^{\prime} \mathrm{N}-171^{\circ} 10^{\prime} \mathrm{W}$ $17^{\circ} 28^{\prime-1}-170^{\circ} 31^{\prime \prime} \mathrm{W}$ $17^{\circ} 28^{\prime} \mathrm{N}-170^{\circ} 31^{\prime} \mathrm{W}$ $17^{\circ} 28^{*} \mathrm{~N}-170^{\circ} 31^{*} \mathrm{~W}$ $17^{\circ} 28^{\prime} \mathrm{N}-170^{\circ} 31^{\circ} \mathrm{W}$ $17^{\circ} 28 \cdot \mathrm{N}-170^{\circ} 31^{\prime} \mathrm{W}$ $17^{\circ} 28^{\prime N}-170^{\circ} 31^{\prime} \mathrm{W}$ $17^{\circ} 28^{1} \mathrm{~N}-170^{\circ} 31^{\circ} \mathrm{W}$ $17^{\circ} 28^{\prime} \mathrm{N}-170^{\circ} 31^{\prime} \mathrm{W}$ $17^{\circ} 28^{\prime} \mathrm{N}-170^{\circ} 32^{\prime} \mathrm{W}$ $17^{\circ} 28^{\prime} \mathrm{N}-170^{\circ} 31^{\prime} \mathrm{W}$ $17^{\circ} 28^{\prime} \mathrm{N}-170^{\circ} 31^{\prime} \mathrm{W}$ $17^{\circ} 28: \mathrm{N}-170^{\circ} 31^{\prime} \mathrm{W}$ $17^{\circ} 28^{\prime} \mathbb{N}-170^{\circ} 31^{\prime} \mathrm{W}$ $17^{\circ} 28 * N-170^{\circ} 31^{\prime} \mathrm{W}$ $17^{\circ} 28^{*} \mathrm{~N}-170^{\circ} 31^{2} \mathrm{~W}$ $17^{\circ} 40^{\prime} \mathrm{N}-169^{\circ} 57^{\prime} \mathrm{W}$ $17^{\circ} 40^{\prime} \mathrm{N}-169^{\circ} 57^{\prime} \mathrm{W}$ $17^{\circ} 42^{\prime} \mathrm{N}-169^{\circ} 47^{\prime} \mathrm{W}$ $18^{\circ} 38^{\prime} \mathrm{N}-166^{\circ} 51^{\prime \prime} \mathrm{W}$ $18^{\circ} 43^{\prime} \mathrm{N}-166^{\circ} 19^{\prime} \mathrm{W}$ $18^{\circ} 48^{\prime} \mathrm{N}-166^{\circ} 19^{\prime} \mathrm{W}$ $18^{\circ} 48^{\prime} \mathrm{N}-166^{\circ} 19^{\circ} \mathrm{W}$ 


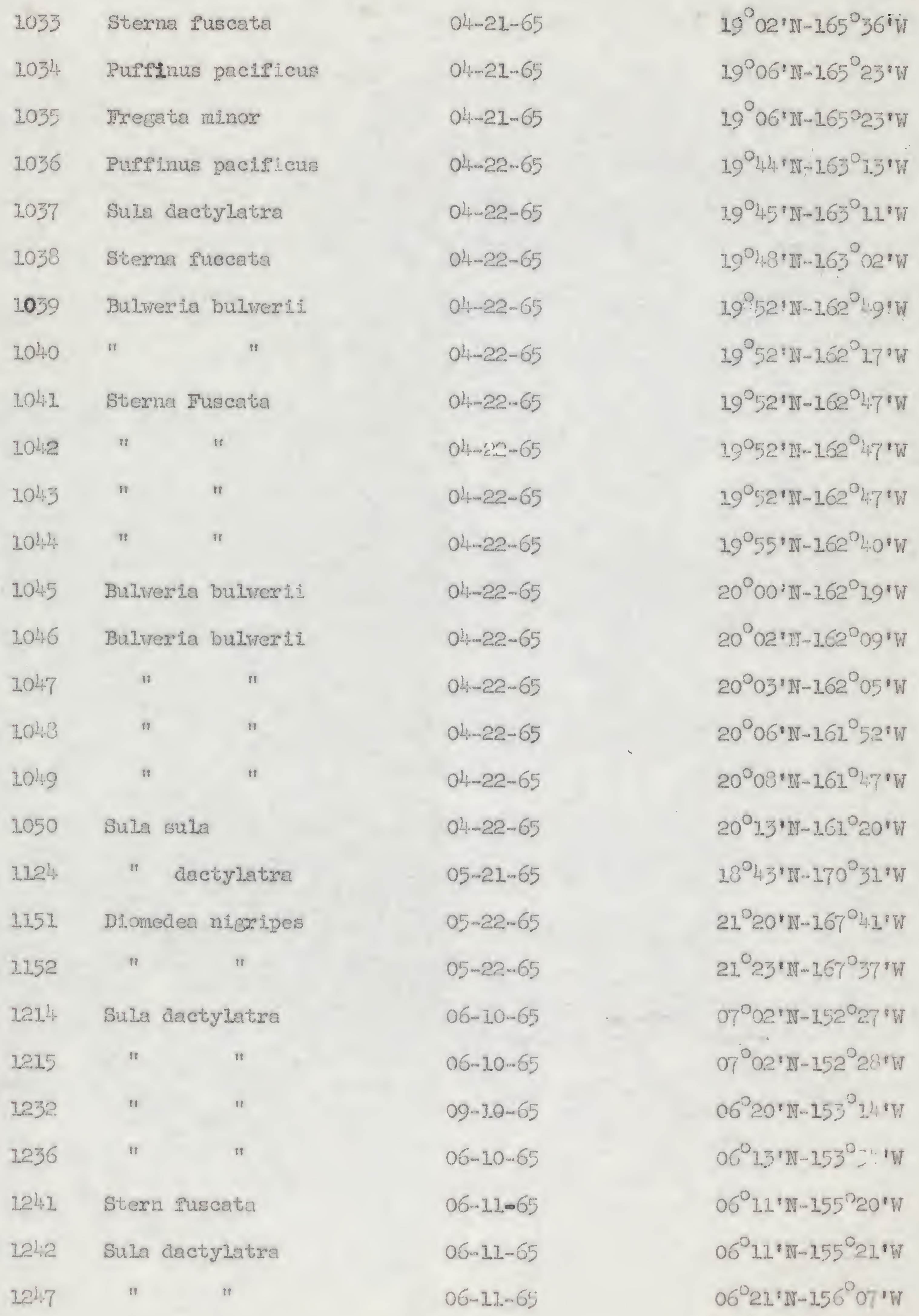


1256 Fregata minor

1261 Puffinus pacificus

1299 Sterna ruscata

$13 \cap 0$

1302

1306 Pufrinus pacteicus

1.308

1314 Fregata minor

1331 Sula sula

1345 Pufeinus pactflcus

1353

1357 Phaethon rubricauda

1358 Sterna fuscata

1359

1360

1361

1419 Pterodroma externa

1420

14.21

1422 Sula inctylatra

1.423 Sterna Puscata

1425 Pterodrona externa

1426 Pufplnus pactelcus

2427

1428

1.430

1431 Pterormoma externa

1432 Puefinus pacificus
$06-12-65$

$06-12-65$

$06-23-65$

06-83-65

$05-23-65$

$06-23-65$

$06-23-65$

$06-24-65$

$06-24-65$

$07-10-65$

$07-10-65$

$07-12 \cdot 65$

$07-11-65$

$07-11-65$

$07-11-65$

$07-1,1-65$

$09-11-65$

$09-11-65$

$09-12-65$

$09-12-65$

$09-12-65$

$09-13-65$

$09-13-65$

$09-13-65$

$09-13-65$

09-14-65

$09-14-65$

$09-14-65$ $07^{\circ} 05^{\prime} \mathrm{N}-158^{\circ} 44^{\circ} \mathrm{W}$

$07^{\circ} 18: N-159^{\circ} 30^{*} \mathrm{~W}$

$19^{\circ} 40^{\prime} \mathrm{N}-163^{\circ} 4,5 \mathrm{~W}$

$19^{\circ} 40^{*} \mathrm{~N}-163^{\circ} 45^{\prime} \mathrm{W}$

$19^{\circ} 49^{\prime} \mathrm{N}-1.65^{\circ} 09^{\prime} \mathrm{W}$

$19^{\circ} 50^{\prime} \mathrm{N}-163^{\circ} \mathrm{O} 4^{\prime} \mathrm{W}$

$19^{\circ} 50^{\circ} \mathrm{N}-163^{\circ} \mathrm{O} 4: \mathrm{W}$

$20^{\circ} 28^{\prime} \mathbb{N}-160^{\circ} 4^{\prime} \mathrm{W}$

$20^{\circ} 4^{*} \mathrm{~N}-159^{\circ} 24^{\prime} \mathrm{W}$

$16^{\circ} 33^{\prime} \mathrm{N}-170^{\circ} 55^{\circ} \mathrm{W}$

$16^{\circ} 25^{\prime} \mathrm{W}-172^{\circ} 31^{.} \mathrm{W}$

$14^{\circ} 38^{*} \mathrm{~N}-173^{\circ} 48^{*} \mathrm{~W}$

$16^{\circ} 04^{\prime} \mathrm{N}-174^{\circ} 11^{\prime} \mathrm{W}$

$15^{\circ} 04^{\prime} \mathrm{N}-174^{\circ} 11^{\prime} \mathrm{W}$

$15^{\circ} 04^{\prime} \mathrm{N}-174^{\circ} 12^{\prime} \mathrm{W}$

$15^{\circ} 04^{\prime} \mathrm{N}-174^{\circ} 11^{\prime} \mathrm{W}$

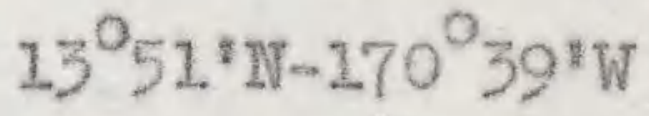

$13^{\circ} 30^{2} \mathrm{~N}-170^{\circ} 48^{2} \mathrm{~W}$

$10^{\circ} 12^{\prime} \mathrm{H}-172^{\circ} 14^{\prime \prime} \mathrm{W}$

$09^{\circ} 10^{\prime} \mathrm{W}-172^{0} 44: \mathrm{W}$

$09^{\circ} 04^{\prime} \mathrm{N}-172^{0} 49^{\prime} \mathrm{W}$

$6^{\circ} 22 ! \mathrm{W}-173^{\circ} 48^{\prime} \mathrm{W}$

$05^{04} \cdot 5^{1} \mathrm{~W}-177^{\circ} 04^{\circ} \mathrm{W}$

$05^{\circ} 45^{\prime} \mathrm{N}-174^{\circ} \mathrm{O} 4^{\circ} \mathrm{W}$

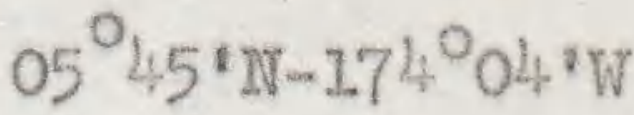

$05^{\circ} 14^{\prime} \mathrm{N}-176^{\circ} 06^{\prime} \mathrm{W}$

$05^{\circ} 14^{2} \mathrm{~N}-176^{\circ} 09^{\prime} \mathrm{W}$

$05^{\circ} 15^{\prime} \mathrm{N}-176^{\circ} 17^{\prime} \mathrm{W}$ 
1433 Puffinus Temulrostris

1434

$n$

14.35

1436

Sterna fuscata

n

$09-14-65$

$09-14-65$

$09-15-65$

$09-15-65$

1437

1438

1439 Sterna fuscata

1440 Puffinus pacificus

$14 \div 1$

tenuirostris

24.42

I443 Pluvialis dominiea

24.4

Sterna fuscata

1445 Anous stolldus

1446

1448

1449

1450

14.51

1452

Sterna fuscata

2453

Arenarla interpres

1454

Sterna Ruscata

1455

n

st

1456 PufPinus pacificus

1457 Sterna fuscata

1458 Puffinus pactificus

1459 Sula sula

14.60 Arenaria interpres

1461

Sula sula
$09-15-65$

$09-15-65$

$09-15-65$

$09-15-65$

$09-16-65$

$09-16-65$

$09-16-65$

$09-16-65$

$09-17-65$

$09-17-65$

$09-27-65$

$09-17-65$

$09-18-65$

$09-18-65$

$09-18-65$

$09-18-65$

$09-19-65$

$09-19-65$

$09-19-65$

$09-19-65$

09-19-65

$10-14-65$

$10-1 \div-65$

$10-06-65$ $05^{\circ} 16^{\prime} \mathrm{N}-176^{\circ} 24 \cdot \mathrm{W}$

$05^{\circ} 17^{\prime} \mathrm{N}-176^{\circ} 26^{\circ} \mathrm{W}$

$02^{\circ} 40^{\prime} \mathrm{N}-176^{\circ} 27^{\prime} \mathrm{W}$

$02^{\circ} 40^{\prime} \mathrm{H}-176^{\circ} 27^{\prime} \mathrm{W}$

$02^{\circ} 33^{\prime} \mathrm{N}-176^{\circ} 25^{\prime} \mathrm{W}$

$02^{\circ} 28^{\prime} \mathrm{N}-176^{\circ} 24^{\prime} \mathrm{W}$

$02^{\circ} 03^{*} \mathrm{~N}-176^{\circ} 24^{*} \mathrm{~W}$

$01^{0} 57^{*} \mathrm{~N} \cdots 176^{\circ} 2^{\prime}: \mathrm{W}$

$00^{\circ} 04^{\prime} \mathrm{S}-176^{\circ} 4^{\circ} 3^{\circ} \mathrm{W}$

$00^{\circ} 06^{\prime} \mathrm{S}-176^{\mathrm{O}} 44_{4} \cdot \mathrm{W}$

$00^{\circ} 11^{\prime} \mathrm{S}-176^{\circ} 49^{\prime} \mathrm{W}$

$00^{\circ} 23^{\prime} \mathrm{S}-176^{\circ} 58^{\prime} \mathrm{W}$

$01^{\circ} 19^{\prime} \mathrm{N}-177^{\circ} 32^{\prime} \mathrm{W}$

$01^{\circ} 19^{\prime} \mathrm{N}-177^{\circ} 32^{\prime} \mathrm{W}$

$01^{\circ} 16^{\prime} \mathrm{m}-176^{\circ} 44 \cdot \mathrm{W}$

$01^{\circ} 15^{\prime} \mathrm{N}-176^{\circ} 16^{\prime} \mathrm{W}$

$00^{\circ} 15^{*} \mathrm{~S}-175^{\circ} 56^{\prime} \mathrm{w}$

$00^{\circ} 15^{\prime} \mathrm{S}-175^{\circ} 56^{\prime} \mathrm{W}$

$00^{\circ} 32^{\prime} \mathrm{S}-175^{\circ} 52^{\prime} \mathrm{W}$

$01^{\circ} 26^{\prime} \mathrm{S}-176^{\circ} 28^{\prime} \mathrm{W}$

$00^{\circ} 15^{\prime} \mathrm{N}-177^{\circ} 33^{*} \mathrm{~W}$

$00^{\circ} 15^{\prime} \mathrm{N}-177^{\circ} 33^{\prime} \mathrm{W}$

$00^{\mathrm{C}} 18 \mathrm{~N}-177^{\circ} 29^{\prime} \mathrm{W}$

$00^{\circ} 25^{\prime} \mathbb{N}-177^{\circ} 15^{\prime} \mathrm{W}$

$00^{\circ} 40^{\prime} \mathrm{N}-176^{\circ} 50^{\prime} \mathrm{W}$

$03^{\circ} 48^{\prime} \mathrm{S}-174^{\circ} 56^{\prime} \mathrm{W}$

$03^{\circ} 4^{\prime} \mathrm{S}-174^{\circ} 59^{\prime} \mathrm{W}$

$13^{\circ} 43^{\prime} \mathrm{S}-170^{\circ} 38^{\prime} \mathrm{W}$ 
1462 Pluvialis dominica

1463 Sterna fuscata

1. 64

1.) 65

$31 \div 66$

1467

12.68 Heteroscelus incanum

1469 Anous stolidus

1170 Puffinus tenuirostris

1471 Pluvialia dominica

1476 Sterna fuscata

1478

1.82 Pterodroms inexpectata

1.85 Decanodroma eastro

1487 Phacthon lepturus

1483

1489 Ocennites occanicus

1490

1.491

1494 Gysis alba

1502 Pterodroma inexpectata

1503

1510 Sterna Ruscata

1515 Oceanites oceantcus

1519 Sterna Fuseata

1521 Sterna Fuscata
$10-06-65$

$10-13-65$

$10-13-65$

$10-13-65$

$10-13-65$

$10-13-65$

$10-13-65$

$10-15-65$

$10-15-65$

$10-? 6 \cdots$

$10-17-65$

$10-17-65$

$10-1.8-65$

$10-18-65$

$10-19-6$

$10-19-65$

$10-19-65$

$10 \cdot 19 \cdot 65$

$10-20 \cdots 65$

10-20-65

$10-20 \cdots 65$

$10-20-65$

$10-22-65$

$10-22-65$ $13045^{\prime} \mathrm{S}-170^{\circ} 37^{\prime} \mathrm{W}$

$06^{\circ} 03^{\prime} \mathrm{s}-173^{\circ} 37^{\prime} \mathrm{V}$

$06^{\circ} 03^{\circ} \mathrm{S}-173037^{\prime \prime} \mathrm{T}$

$06^{2} 03^{\prime} \mathrm{S}-173^{2} 45^{\prime} \mathrm{W}$

$05^{\circ} 03^{\prime} \mathrm{S}-173^{\circ} 45^{\prime} \mathrm{W}$

$06^{\circ} 03^{1} \mathrm{~S}-173^{\circ} 45^{\mathrm{W}}$

$05^{\circ} 56^{\circ} \mathrm{S}-173^{\circ} 48 \mathrm{~W}$

$00^{\circ} 13 \cdot 5-176^{\circ} 09^{\prime} \mathrm{W}$

$00^{\circ} 10^{\prime} \mathrm{N}-176^{\circ} 26^{\circ} \mathrm{W}$

$01^{\circ} 27^{\prime} \mathrm{N}-176^{\circ} 15^{\prime} \mathrm{W}$

$00^{\circ} 02^{1} \mathrm{~S}-176057^{\circ} \mathrm{W}$

$00^{\circ} 20^{1} \mathrm{~N}-1.76^{\circ}, \mathrm{S}^{\mathrm{n}} \mathrm{W}$ $02^{0} 00^{2} \mathrm{~m}-177^{\circ} \mathrm{j0 \textrm {W }}$ $01^{0} 39^{\prime} \mathrm{N}-170^{\circ} 07^{\prime} \mathrm{W}$

$00^{\circ} 20^{\prime} \mathrm{H}-177^{\circ} 28^{\prime} \mathrm{W}$ $00^{\circ} 20^{\prime} 11-177^{\circ} 25^{\prime} \mathrm{W}$ $00^{\circ} 20^{\prime 14}-177^{\circ} 20^{\prime} \mathrm{W}$ $00^{\circ} 26 \cdot \mathrm{s}-177^{\circ} 38^{\prime} \mathrm{W}$ $00026^{\circ} \mathrm{S}-177^{0} 37^{\prime} \mathrm{W}$ $00927^{\circ} \mathrm{S}-277^{\circ} 35^{\circ} \cdot 4$ $00^{\circ} 27^{\prime} \mathrm{S}-177^{0} 36^{\prime} \mathrm{W}$ $00^{\circ} 27^{\prime} \mathrm{S}-177^{\circ} 36^{\prime} \mathrm{W}$ $00^{\circ} 34^{\prime} \mathrm{N}-176^{\circ} 20^{\prime} \mathrm{W}$ $00^{\circ} 14 \cdot \mathrm{N}-175^{\circ} 52^{\prime} \mathrm{W}$ 


\begin{tabular}{|c|c|c|c|}
\hline 1527 & Phaethon lepturus & $10-23-65$ & $01^{\circ} 29^{\prime} \mathrm{S}-173^{\circ} 08^{\prime} \mathrm{W}$ \\
\hline 1531 & Sterna paradisaea & $10-23-65$ & $01052^{\prime} \mathrm{s}-172^{05} 3^{\prime} \mathrm{W}$ \\
\hline 1533 & Sula sula & $10-24-65$ & $\left.02^{\circ} 22^{\prime} \mathrm{s}-170^{\circ}\right)_{4} 3^{\prime} \mathrm{W}$ \\
\hline 1542 & Sula sula & $10-25-65$ & $00^{\circ} 17^{\prime} \mathrm{s}-167^{\circ} 42^{\prime} \mathrm{W}$ \\
\hline 1547 & Sula dactylatra & $10-26-65$ & $0007^{\prime} \mathrm{s}-164^{\circ} 53^{\prime} \mathrm{W}$ \\
\hline 1548 & Sula dactylatra & $10-26-65$ & $00^{\circ} 07^{\prime} \mathrm{s}-164^{\circ} 53^{\prime} \mathrm{W}$ \\
\hline 1551 & Gygis Alba & $10-26-65$ & $00^{\circ} 07^{\prime} \mathrm{s}-164^{\circ} 53^{\prime} \mathrm{W}$ \\
\hline 1552 & Oceanodroma Ieucorhoa & $10-26-65$ & $00^{\circ} 03^{\prime} \mathrm{N}-164^{\circ} 14^{\prime} \mathrm{W}$ \\
\hline 1567 & Oceanodroma leucorhoa & $11-01-65$ & $06^{\circ} 41^{\prime} \mathrm{N}-157^{\circ} 29^{\prime} \mathrm{W}$ \\
\hline 1570 & Pterodroma Alba & $11-01-65$ & $06^{\circ} 53^{\prime} \mathrm{N}-157^{\circ} 27^{\prime} \mathrm{W}$ \\
\hline 1572 & Pterodroma Externa & $11-01-65$ & $07^{\circ} 05^{\prime} \mathrm{N}-157^{\circ} 28^{\prime} \mathrm{W}$ \\
\hline 1572 & Pterodroma hypoleuca & $11-01-65$ & $07^{\circ} 11^{\prime} \mathrm{N}-157^{\circ} 27^{\prime} \mathrm{W}$ \\
\hline 1573 & Pterodroma Externa & $11-03-65$ & $14^{\circ} 08^{\prime} \mathrm{N}-157^{\circ} 39^{\prime} \mathrm{W}$ \\
\hline 1574 & Pterodroma hypoleuca & $11-03-65$ & $14^{\circ} 11^{\prime} \mathrm{N}-157^{\circ} 38^{\prime} \mathrm{W}$ \\
\hline 1575 & Pterodroma hypoleuca & $12-04-65$ & $18046^{\prime} \mathrm{N}-157^{\circ} 48^{\prime} \mathrm{W}$ \\
\hline 1576 & Oceanodroma leucorhoa & $11-04-65$ & $18^{\circ} 55^{\prime} \mathrm{N}-157^{\circ} 4^{\prime} 8^{\prime} \mathrm{W}$ \\
\hline 5592 & Anous stolidus & $06-01-65$ & Canton Atol1, Phoenix Islands \\
\hline 5756 & Sula dactylatra & $09-16-65$ & Howland Island, Pacific Ocean \\
\hline 5758 & Anous Minutus & $09-16-65$ & $n \quad n \quad n$ \\
\hline 5760 & Sula sule & $09-22-65$ & McKean Is., Phoenix Islands \\
\hline 5764 & Phaethon rubricauda & $09-23 \cdot 65$ & $"$ \\
\hline 3 & Fregata Ariel & $09-23-65$ & $" \quad "$ \\
\hline & Sula dactylatra & $09-23-65$ & $"$ \\
\hline 5767 & Sula dactylatra & $09-23-65$ & 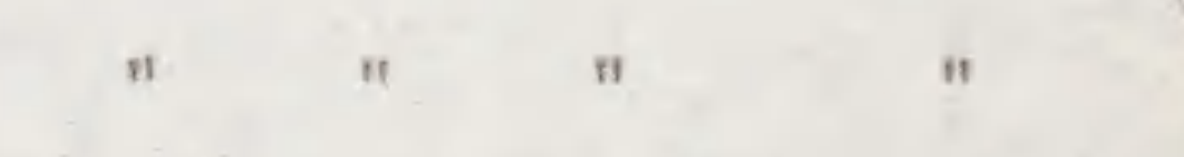 \\
\hline & Puffinus paciflcus & $09-24-65$ & Hul1 Is., Phoenix Islanda \\
\hline & Heteroscelus incanum & $09-30-65$ & Phoenix Is.," \\
\hline
\end{tabular}


5770 Erolia Melanotos

5771 Sula leucogaster

5806 Sula dactylatra

5807 Erolla Melanotos

$5808 \ldots$ " *

5809 Sula sula

5811. Sterna Fuscata

5812

5814 Erolia Acuminata

5815 Numenius Tahitiensis

5816 Heteroscelus incanum

5818 Limoga lapponica

5819 Erolia Acuminata

$5820 \quad$ Vini kuhli

5821 Thalasseus bergi1

5822 Vini kuhli

5823 Gygis albe

5824 Thalasseus bergil

5825

5826 Pluvialis domintca

5827

5828 Numenius Tahitiensis

5829

58,1 Sula dactylatra

583 Spatula clypeata
$09-30-65$

$09-30-65$

$09-30-65$

$10-02-65$

$10-02-65$

$10-02-65$

$09-24-65$

$09-24-65$

$09-18-65$

$09-19-65$

$09-16-65$

$10-19-65$

$10-20-65$

$10-28-65$

$10-28-65$

$10-28-65$

$10-28-65$

10-28-65

$10-28-65$

$10-28-65$

$10-28-65$

$10-28-65$

$10-28-65$

$10-29-65$

$10-29-65$

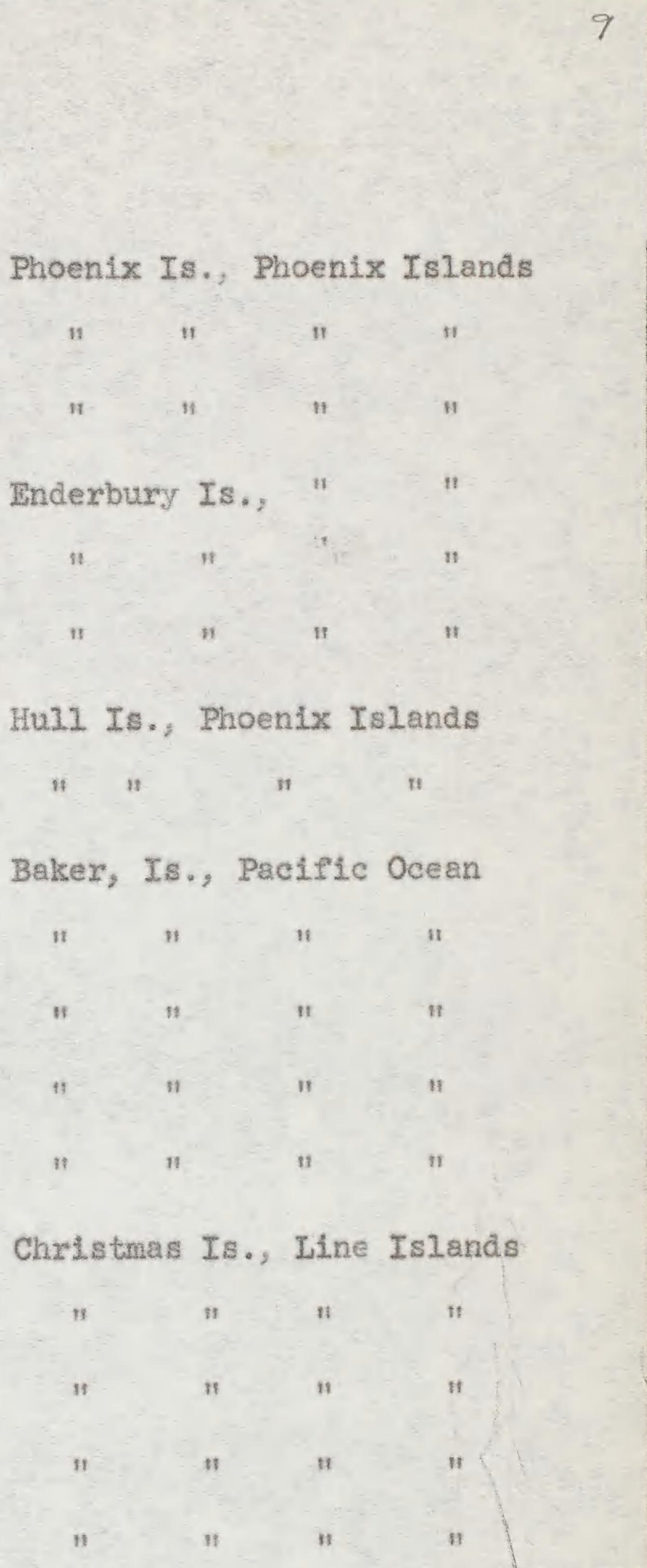

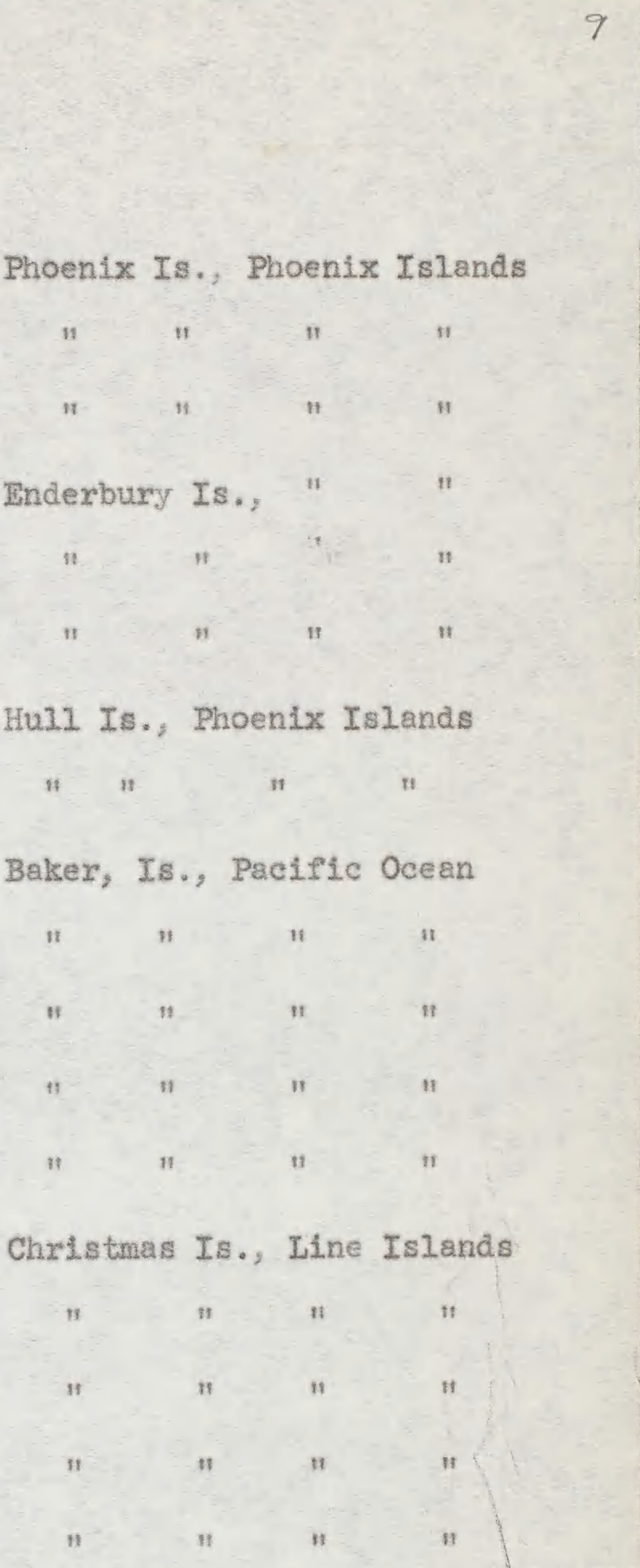

n

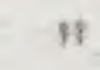

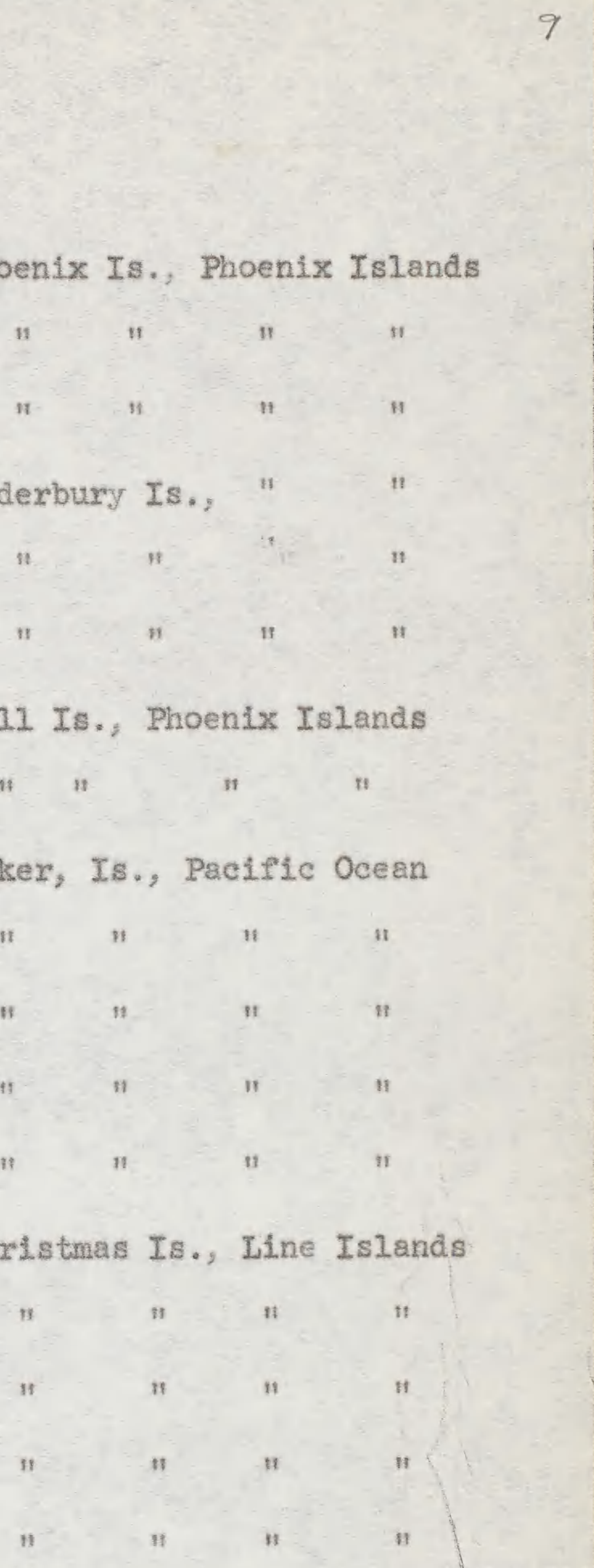

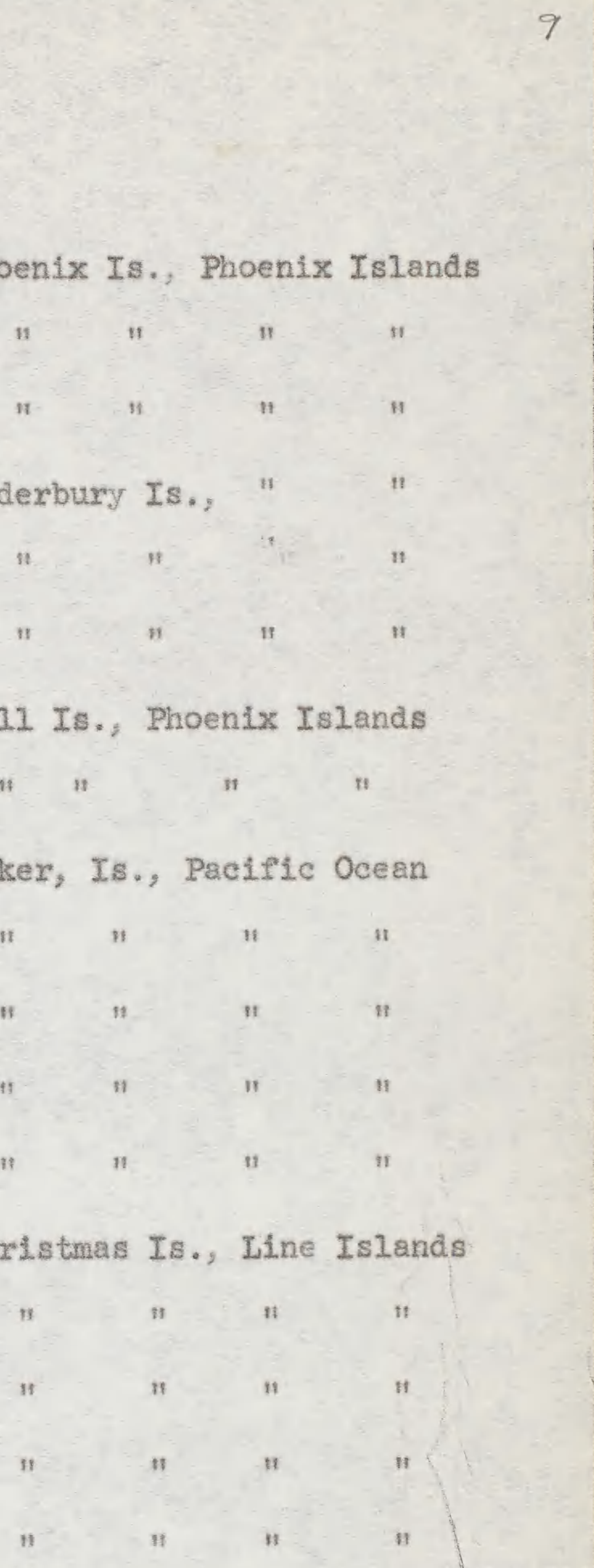

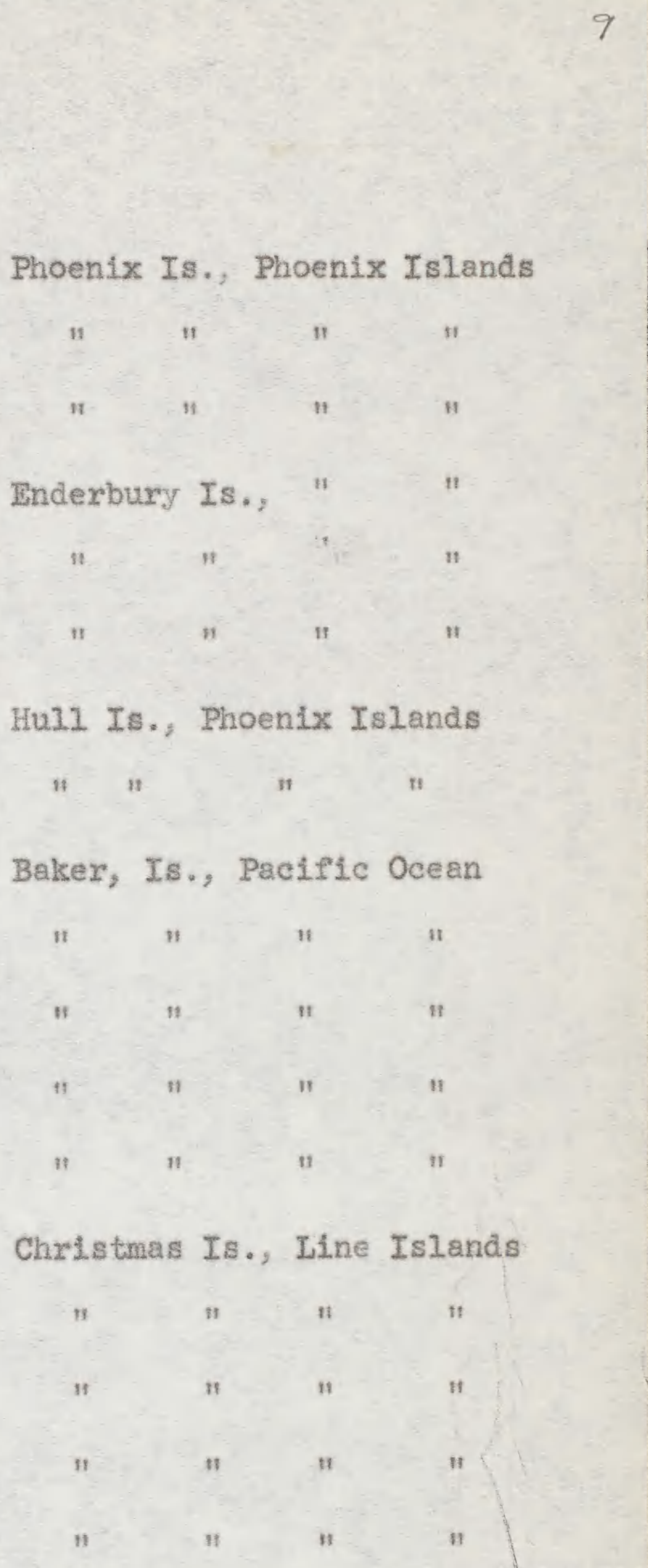

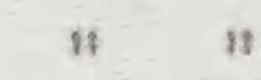

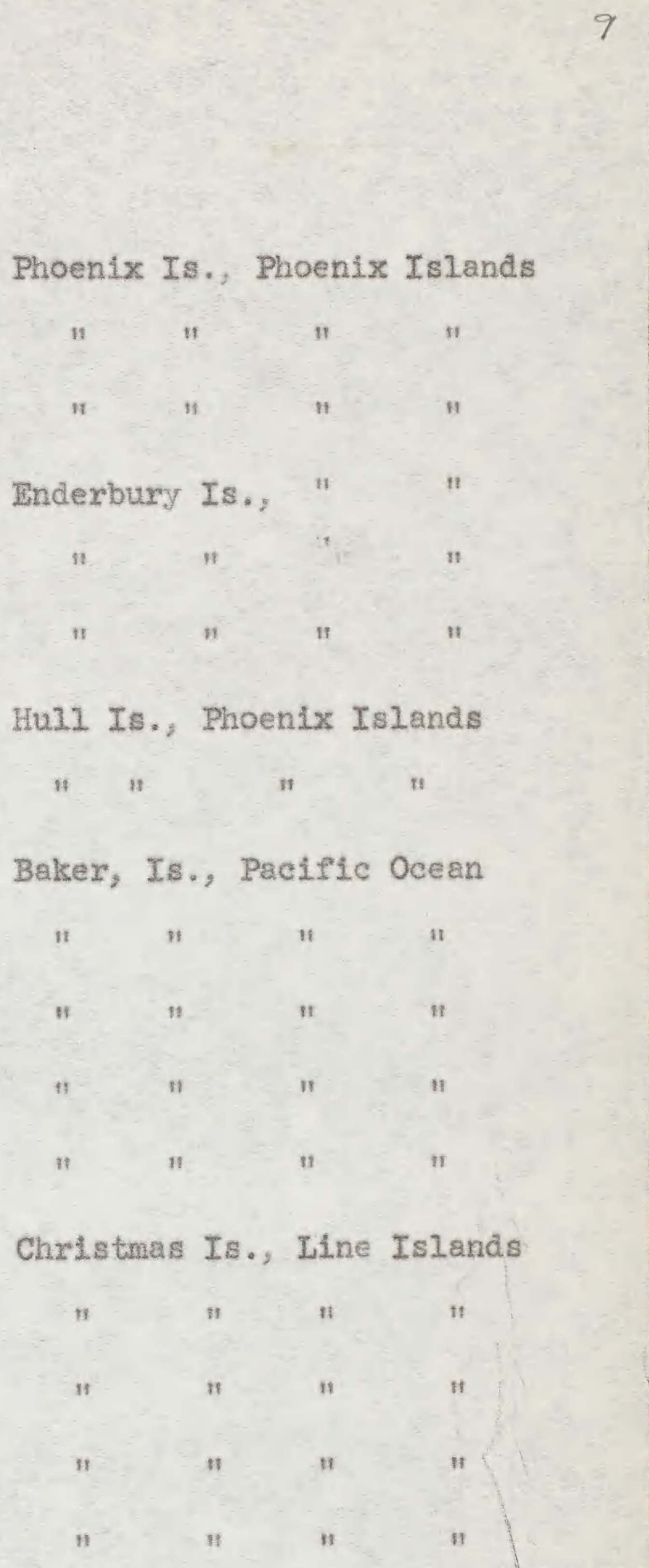

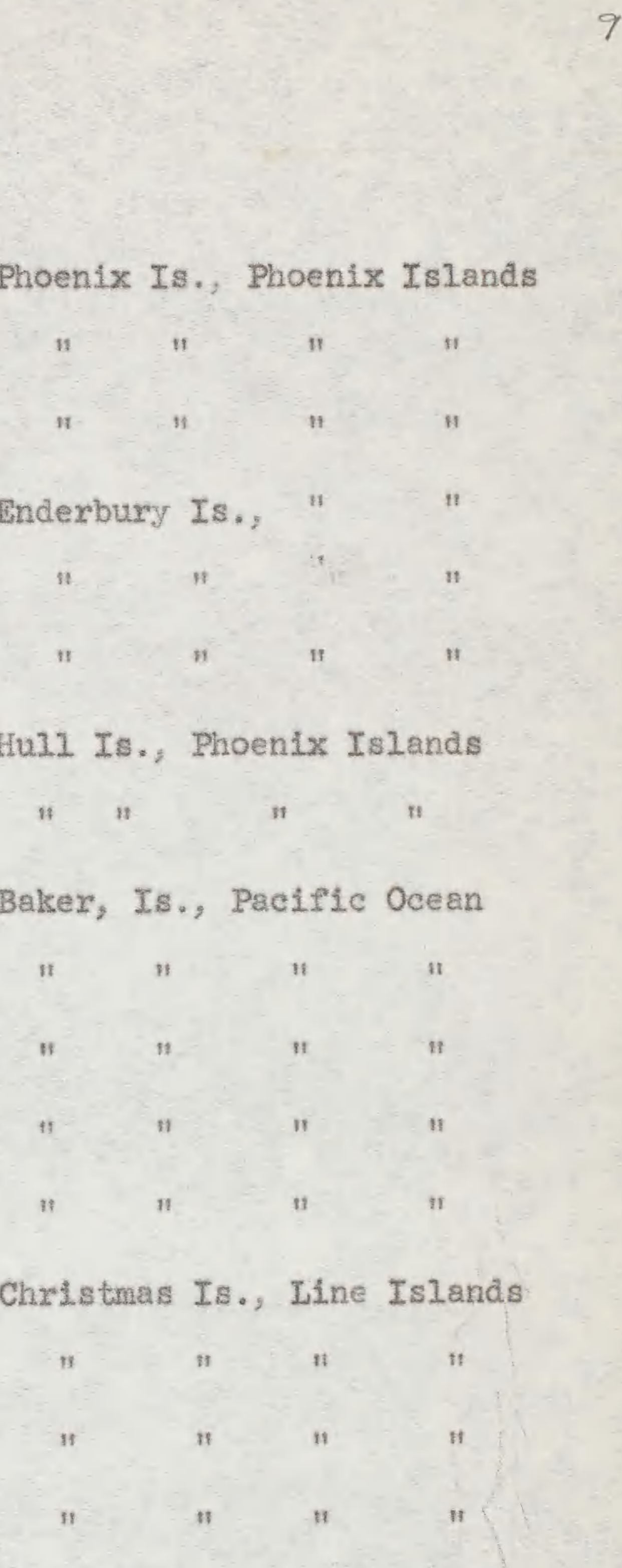

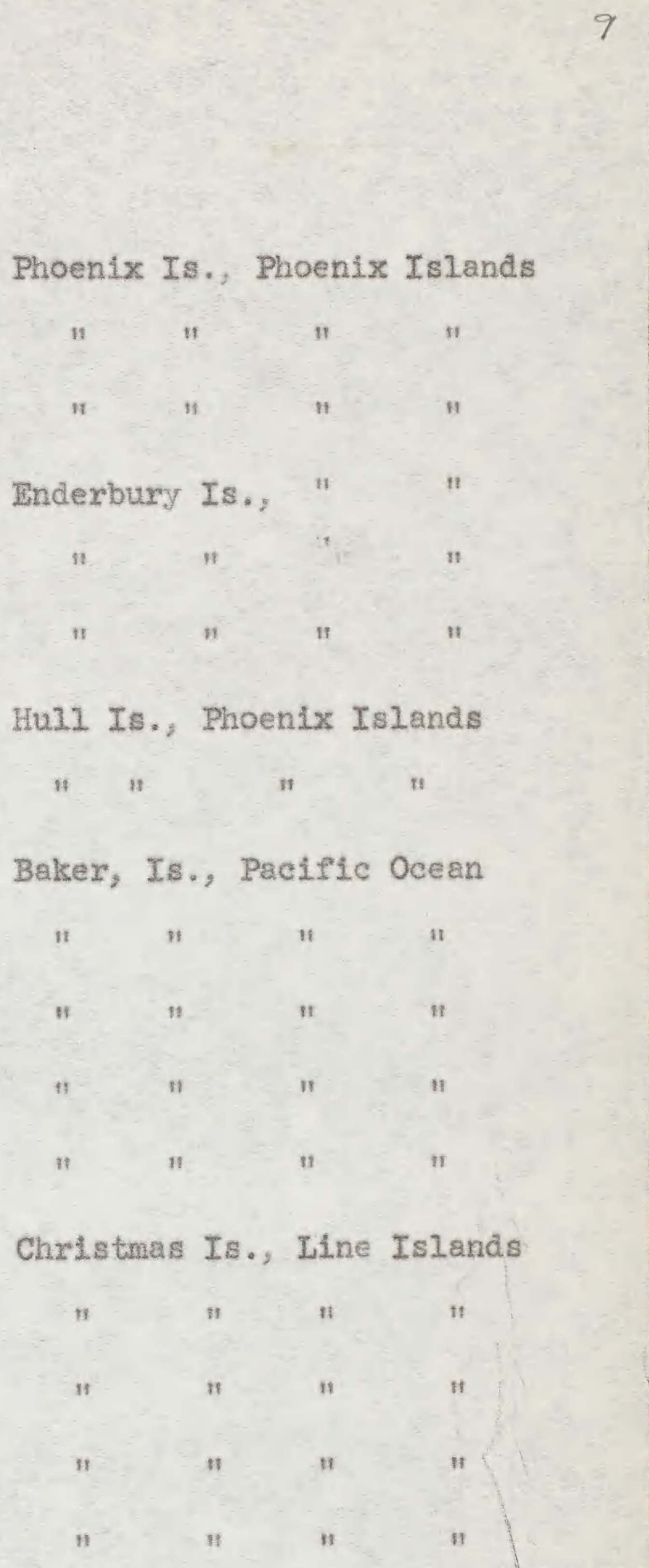

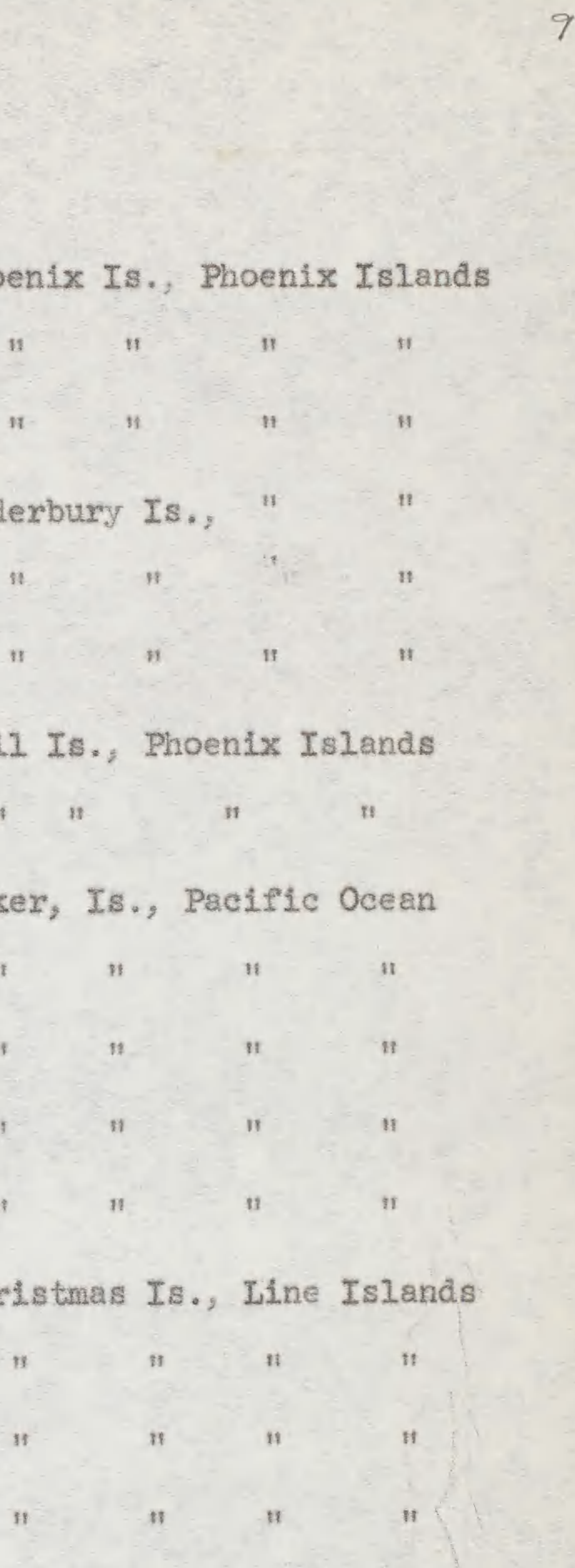

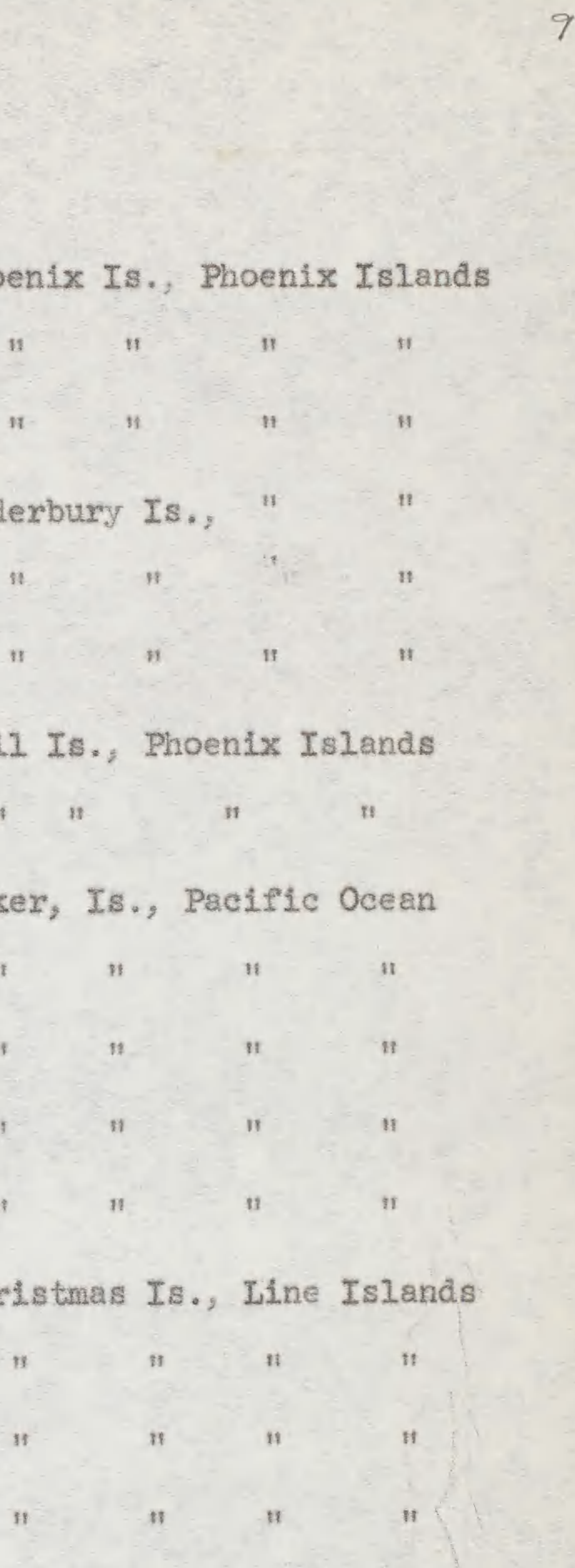

$$
H
$$

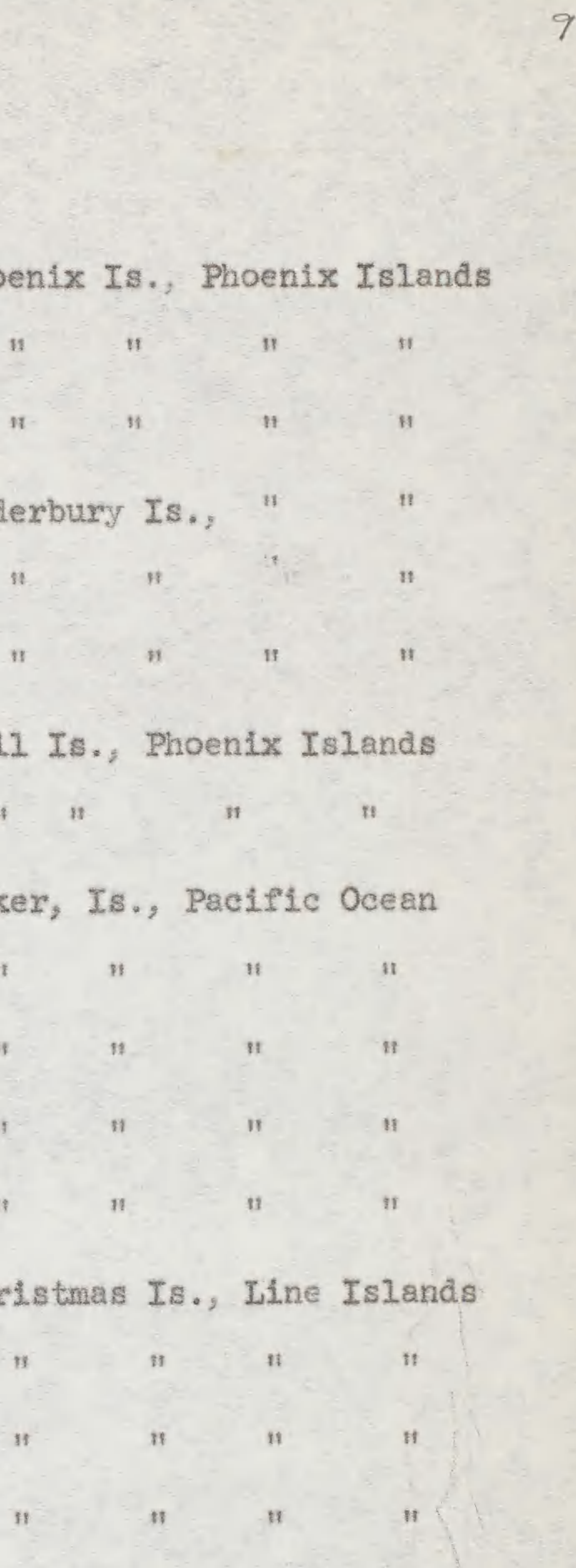

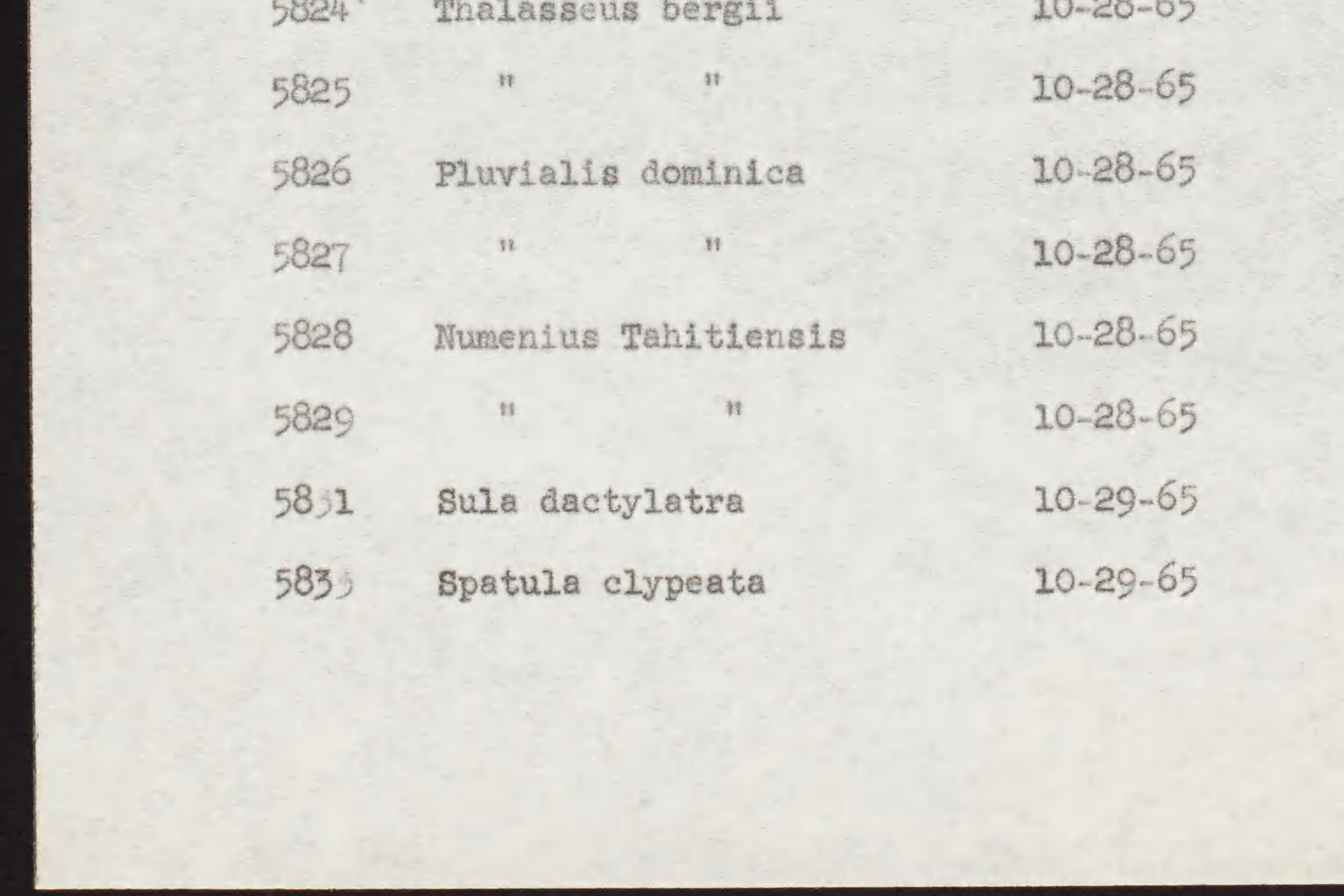


5863 Pterodroma Alba

5864 Pterodroma Alba

5865 Pterodroma Alba

5867 Sula sula

20282 Totanus Flavipes

20289 Sula sula

20440 Gaygis Alba

20477 Phaethon rubricauda

20478 Sula sula

20481 Fregata Minor

20482 Fregata Minor

20485 Phaethon rubricauda

30031 Axenaria interpres

3003

15008 Mimus polyglottos
$10.30-65$

$10-30-60$

$10-30-65$

$10-30-65$

$09-02-64$

$09-11-64$

$02-25-65$

$05-25-65$

$05-27-65$

$06-03-65$

$06-03-65$

$07.01-65$

$10-15-64$

$10-15-64$

$08-18-65$
Christmas Is., line IElands

Green Is., Kure Atold, Hawa1

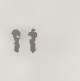

II

$\because$

is

it

11

II

Bikar Atoll, Marsiall Islanas

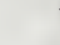

French Frigate Shoals, Hawaii 
SOSC REF. NO. 229

Sta. No. 0951 Species Sterna fuscata

POBSP. NO. 10

\begin{tabular}{|c|c|c|c|c|c|c|c|c|c|}
\hline $\begin{array}{l}\text { Lat \& } \\
\text { Long. }\end{array}$ & Date & $\begin{array}{l}\text { Time } \\
\text { Caught }\end{array}$ & Squid & $\begin{array}{l}\text { Squid } \\
\text { Beaks }\end{array}$ & Fish & $\begin{array}{c}\text { Fish } \\
\text { Remains }\end{array}$ & $\begin{array}{l}\text { Parasitic } \\
\text { Animals }\end{array}$ & Crustacea & $\begin{array}{c}\text { Other } \\
\text { Animals }\end{array}$ \\
\hline$\frac{11 \cdot 31 \cdot \mathrm{N}}{150^{\circ} 31 \cdot \mathrm{W}}$ & $\begin{array}{l}\text { April } \\
5, \\
65\end{array}$ & $\begin{array}{l}\text { Pacific } \\
\text { Ocoan }\end{array}$ & & 9 & & - & & & \\
\hline
\end{tabular}

Parasitic Animals:

Notes on Squid:

lots: 2 squid beaks

digested remains

Notes on Fish:

General Notes:

Digested remains beyond identification.

PACIFIC OCEANOGRAPHIC BIOLOGICAL SURVEY PROGRAM

SMITHSONIAN OCEANOGRAPHIC SORTING CENTER—SMITHSONIAN INSTITUTION

$\operatorname{Si-USNM-830}$

P. Grisharn 8/1/66

\%

\begin{tabular}{|c|c|c|c|c|c|c|c|c|c|}
\hline $\begin{array}{l}\text { Lat \& } \\
\text { Long. }\end{array}$ & Date & $\begin{array}{l}\text { Time } \\
\text { Caught }\end{array}$ & Squid & $\begin{array}{l}\text { Squid } \\
\text { Beaks }\end{array}$ & Fish & $\begin{array}{c}\text { Fish } \\
\text { Remains }\end{array}$ & $\begin{array}{l}\text { Parasitic } \\
\text { Animals }\end{array}$ & Crustacea & $\begin{array}{l}\text { Other } \\
\text { Animals }\end{array}$ \\
\hline $\begin{array}{l}09^{\circ} 36^{\prime} \mathrm{N} \\
152^{\circ} \cdot 43^{\prime} \mathrm{W}\end{array}$ & $\begin{array}{l}\text { Apr11 } \\
6, \\
65\end{array}$ & $\begin{array}{l}\text { Pacific } \\
\text { Ocean }\end{array}$ & & 2 & & - & & & \\
\hline
\end{tabular}

Parasitic Animals:

Notes on Squid:

lots: 2 squid beaks

digested remains

Notes on Fish:

General Notes:

Digested remains beyond identification.

PACIFIC OCEANOGRAPHIC BIOLOGICAL SURVEY PROGRAM

SMITHSONIAN OCEANOGRAPHIC SORTING CENTER—SMITHSONIAN INSTITUTION

$\underset{7-65}{5 !-U 8 N M A-830}$

P. Grisham 8/30/66 
SOSC REF. NO. 229

\begin{tabular}{|c|c|c|c|c|c|c|c|c|c|}
\hline $\begin{array}{l}\text { Lat \& } \\
\text { Long. }\end{array}$ & Date & $\begin{array}{l}\text { Time } \\
\text { Caught }\end{array}$ & Squid & $\begin{array}{l}\text { Squid } \\
\text { Beaks }\end{array}$ & Fish & $\begin{array}{c}\text { Fish } \\
\text { Remains }\end{array}$ & $\begin{array}{l}\text { Parasitic } \\
\text { Animals }\end{array}$ & Crustacea & $\begin{array}{l}\text { Other } \\
\text { Animals }\end{array}$ \\
\hline $\begin{array}{l}09^{\circ} 27^{\circ} \mathrm{N} \\
152^{\circ} 47^{\circ} \mathrm{W}\end{array}$ & $\begin{array}{l}\text { April } \\
26, \\
65\end{array}$ & $\begin{array}{l}\text { Pachfic } \\
\text { Ocean }\end{array}$ & & 124 & & - & & & \\
\hline
\end{tabular}

Parasitic Animals:

lots: 2 squid beaks

Notes on Squid:

Squid beaks are of various sizes.

digested remains

Notes on Fish:

General Notes:

Digested remains with squid beak fragments.

PACIFIC OCEANOGRAPHIC BIOLOGICAL SURVEY PROGRAM

SMITHSONIAN OCEANOGRAPHIC SORTING CENTER—SMITHSONIAN INSTITUTION

P. Grisham 8/15/66

$\underset{7-65}{\operatorname{si-0.MNM-ะ30~}}$

要

\begin{tabular}{|c|c|c|c|c|c|c|c|c|c|}
\hline \multirow{2}{*}{$\begin{array}{l}\text { Sta. No. } \\
\text { Lat \& } \\
\text { Long. }\end{array}$} & \multicolumn{2}{|l|}{0954} & Species & \multicolumn{3}{|c|}{ Puffinus pacificus } & \multicolumn{2}{|c|}{$\begin{aligned} \text { SOSC REF. NO. } & 229 \\
\text { POBSP. NO. } & 10\end{aligned}$} & \\
\hline & Date & $\begin{array}{c}\text { Time } \\
\text { Caught }\end{array}$ & Squid & $\begin{array}{l}\text { Squid } \\
\text { Beaks }\end{array}$ & Fish & $\begin{array}{c}\text { Fish } \\
\text { Remains }\end{array}$ & $\begin{array}{l}\text { Parasitic } \\
\text { Animals }\end{array}$ & Crustacea & $\begin{array}{l}\text { Other } \\
\text { Animals }\end{array}$ \\
\hline $\begin{array}{l}09^{\circ} 27^{\prime} \mathrm{N} \\
151^{\circ} 47^{\prime} \mathrm{W}\end{array}$ & $\begin{array}{l}\text { April } \\
6, \\
65\end{array}$ & $\begin{array}{l}\text { Pacific } \\
\text { Ocean }\end{array}$ & 1 & 9 & & - & & & \\
\hline
\end{tabular}

Parasitic Animals:

lots: 3 squid

Notes on Squid: Good condition.

digested remains

squid beaks

Notes on Fish:

General Notes:

PACIFIC OCEANOGRAPHIC BIOLOGICAL SURVEY PROGRAM SMITHSONIAN OCEANOGRAPHIC SORTING CENTER_-SMITHSONIAN INSTITUTION SMITHSONIAN OCEANOGRAPHIC SORTING CENTER_P. Grisham 8/23/66 


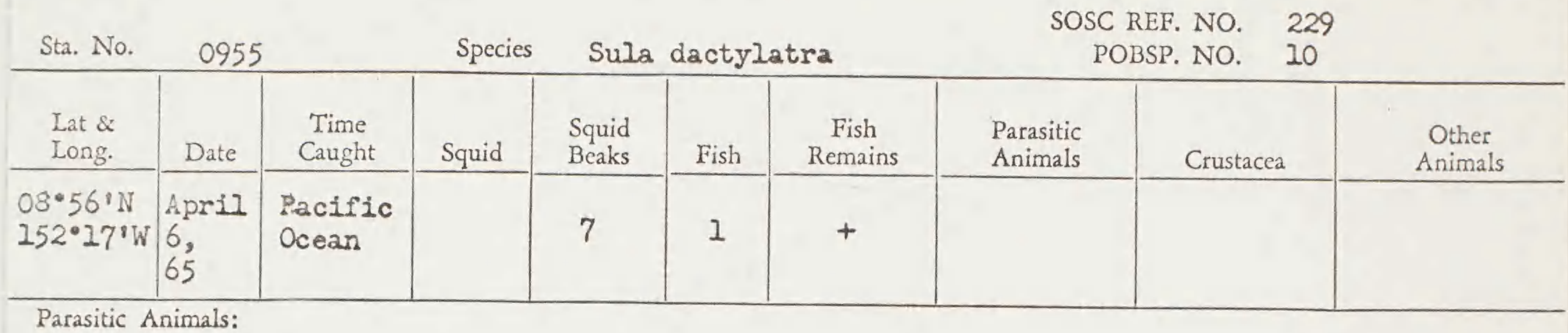

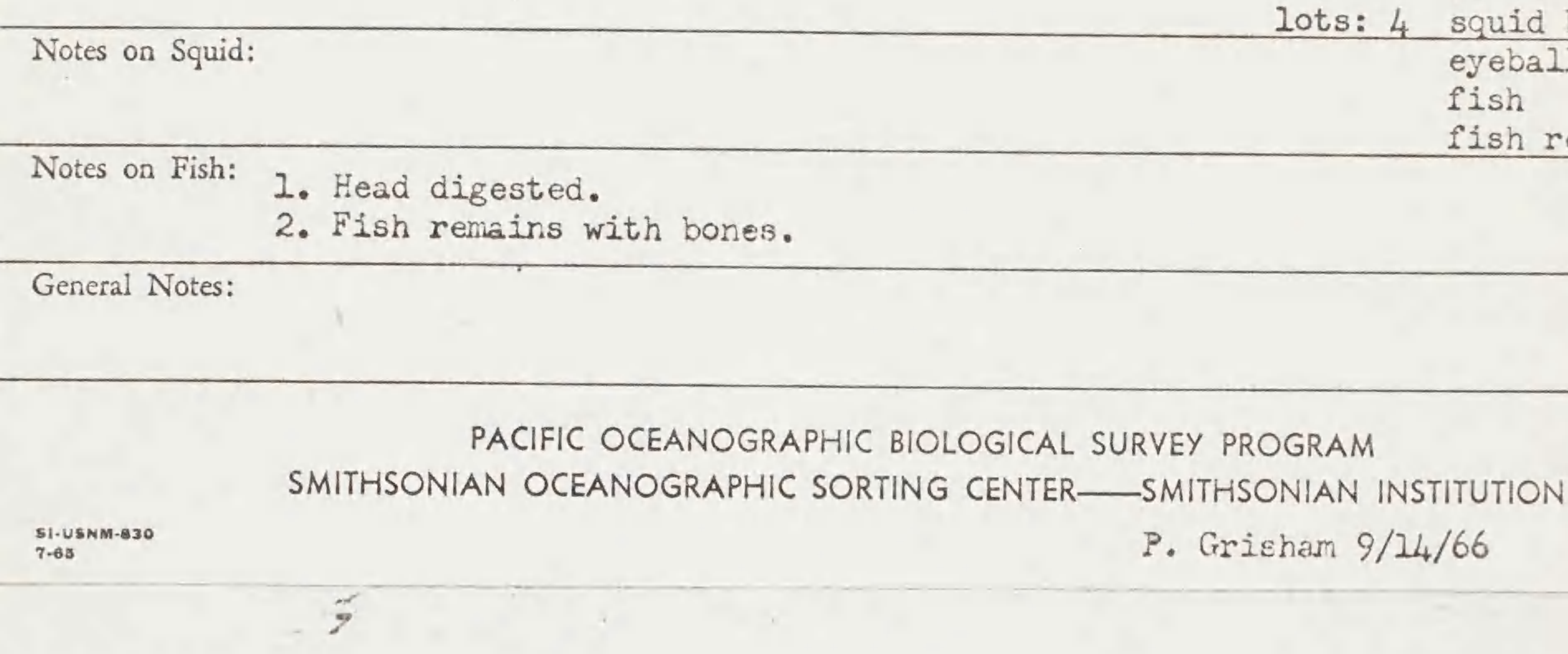

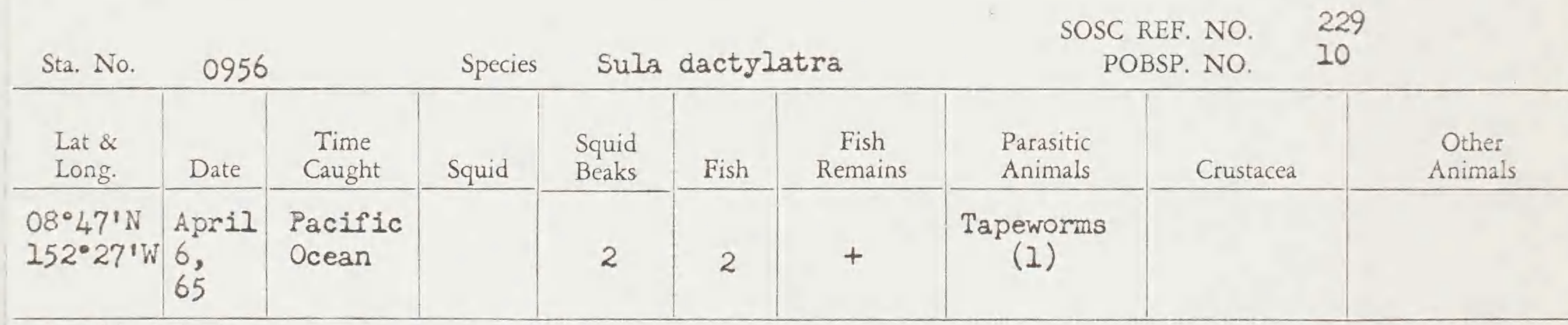

Parasitic Animals:

\section{Notes on Squid:}

Notes on Fish: 1. Fish remains with bones.
2. Head digested.

General Notes:

2. Head digested.
Iots: 4 squid beaks

fish

fish remains

tapeworms

PACIFIC OCEANOGRAPHIC BIOLOGICAL SURVEY PROGRAM SMITHSONIAN OCEANOGRAPHIC SORTING CENTER—SMITHSONIAN INSTITUTION 


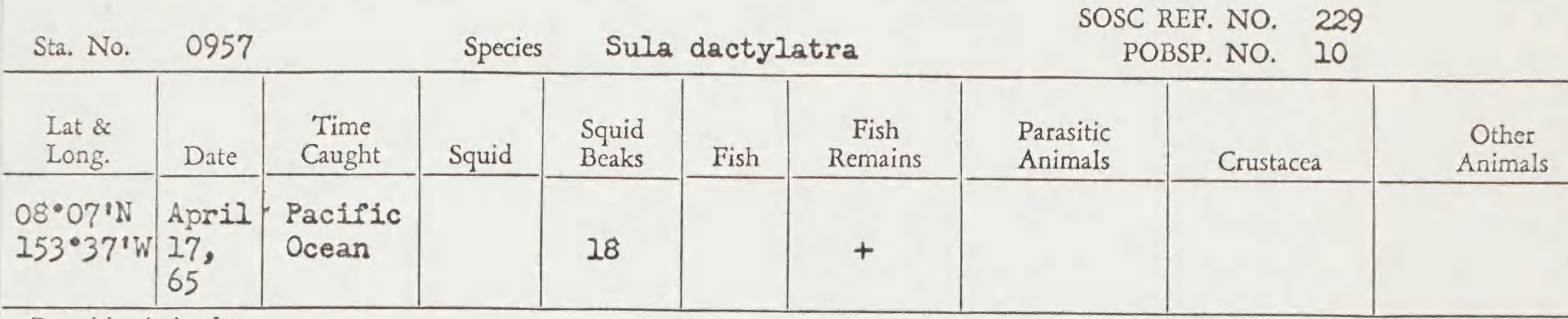

Parasitic Animals:

Notes on Squid:

lots: 2 fish remains

squid beaks

Notes on Fish:

Fish remains with bones.

General Notes:

PACIFIC OCEANOGRAPHIC BIOLOGICAL SURVEY PROGRAM

SMITHSONIAN OCEANOGRAPHIC SORTING CENTER—SMITHSONIAN INSTITUTION

51.USNM-830 P. Grisham 8/24/66

7-65

3.

Sta. No. $0958 \quad$ Species Sula dactylatra

SOSC REF. NO, 229

POBSP. NO. 10

\begin{tabular}{|c|c|c|c|c|c|c|c|c|c|}
\hline $\begin{array}{l}\text { Lat \& } \\
\text { Long. }\end{array}$ & Date & $\begin{array}{l}\text { Time } \\
\text { Caught }\end{array}$ & Squid & $\begin{array}{l}\text { Squid } \\
\text { Beaks }\end{array}$ & Fish & $\begin{array}{c}\text { Fish } \\
\text { Remains }\end{array}$ & $\begin{array}{l}\text { Parasitic } \\
\text { Animals }\end{array}$ & Crustacea & $\begin{array}{l}\text { Other } \\
\text { Animals }\end{array}$ \\
\hline $\begin{array}{l}08^{\circ} 07^{\prime} \mathrm{N} \\
153^{\circ} 37^{\prime} \mathrm{W}\end{array}$ & $\begin{array}{l}\text { April } \\
17, \\
65\end{array}$ & $\begin{array}{l}\text { Pacific } \\
\text { Ocoan }\end{array}$ & & & & - & $\begin{array}{c}\text { Tapeworms } \\
\text { (15) }\end{array}$ & & \\
\hline
\end{tabular}

Parasitic Animals:

Notes on Squid:

lots: 2 tapeworms

digested remains

Notes on Fish:

General Notes:

Digested remains beyond identification.

PACIFIC OCEANOGRAPHIC BIOLOGICAL SURVEY PROGRAM

SMITHSONIAN OCEANOGRAPHIC SORTING CENTER_-SMITHSONIAN INSTITUTION

Si.USNM-30

P. Grisham 8/29/66 
SOSC REF. NO. 229

\begin{tabular}{|c|c|c|c|c|c|c|c|c|c|}
\hline $\begin{array}{l}\text { Lat \& } \\
\text { Long. }\end{array}$ & Date & $\begin{array}{l}\text { Time } \\
\text { Caught }\end{array}$ & Squid & $\begin{array}{l}\text { Squid } \\
\text { Beaks }\end{array}$ & Fish & $\begin{array}{c}\text { Fish } \\
\text { Remains }\end{array}$ & $\begin{array}{l}\text { Parasitic } \\
\text { Animals }\end{array}$ & Crustacea & $\begin{array}{c}\text { Other } \\
\text { Animals }\end{array}$ \\
\hline $\begin{array}{l}08^{\circ} 09^{\prime} \mathrm{N} \\
153^{\circ} 43^{\prime} \mathrm{W}\end{array}$ & $\begin{array}{l}\text { Aprit } \\
7, \\
65\end{array}$ & $\begin{array}{l}\text { Pactfic } \\
\text { Ocean }\end{array}$ & & 5 & & - & & & \\
\hline
\end{tabular}

Parasitic Animals:

lots: 2 squid beaks

Notes on Squid: digested remains

Notes on Fish:

General Notes:

Digested remains beyond identification.

PACIFIC OCEANOGRAPHIC BIOLOGICAL SURVEY PROGRAM

SMITHSONIAN OCEANOGRAPHIC SORTING CENTER—SMITHSONIAN INSTITUTION

Si. USNM- 830

P. Grisham 8/29/66

7-6s

SOSC REF. NO. 229

POBSP. NO. 10

\begin{tabular}{|c|c|c|c|c|c|c|c|c|c|}
\hline $\begin{array}{l}\text { Lat \& } \\
\text { Long. }\end{array}$ & Date & $\begin{array}{l}\text { Time } \\
\text { Caught }\end{array}$ & Squid & $\begin{array}{l}\text { Squid } \\
\text { Beaks }\end{array}$ & Fish & $\begin{array}{c}\text { Fish } \\
\text { Remains }\end{array}$ & $\begin{array}{l}\text { Parasitic } \\
\text { Animals }\end{array}$ & Crustacea & $\begin{array}{l}\text { Other } \\
\text { Animals }\end{array}$ \\
\hline $\begin{array}{r}08^{\circ} 28^{\prime} \mathrm{N} \\
153^{\circ} 35^{\prime} \mathrm{N}\end{array}$ & $\begin{array}{l}\text { April } \\
7, \\
65\end{array}$ & $\begin{array}{l}\text { Pacific } \\
\text { Ocean }\end{array}$ & & 18 & & + & & & \\
\hline
\end{tabular}

Parasitic Animals:

Iots: 2 squid beaks

Notes on Squid:

fish remains

Notes on Fish:

Fish remains with bones.

General Notes:

PACIFIC OCEANOGRAPHIC BIOLOGICAL SURVEY PROGRAM

SMITHSONIAN OCEANOGRAPHIC SORTING CENTER—SMITHSONIAN INSTITUTION 
SOSC REF. NO. 229

\begin{tabular}{|c|c|c|c|c|c|c|c|c|c|}
\hline $\begin{array}{l}\text { Lat \& } \\
\text { Long. }\end{array}$ & Date & $\begin{array}{l}\text { Time } \\
\text { Caught }\end{array}$ & Squid & $\begin{array}{l}\text { Squid } \\
\text { Beaks }\end{array}$ & Fish & $\begin{array}{c}\text { Fish } \\
\text { Remains }\end{array}$ & $\begin{array}{l}\text { Parasitic } \\
\text { Animals }\end{array}$ & Crustacea & $\begin{array}{l}\text { Other } \\
\text { Animals }\end{array}$ \\
\hline $\begin{array}{l}08^{*} 28^{\prime} \mathrm{N} \\
153^{\circ} 35^{\prime} \mathrm{W}\end{array}$ & $\begin{array}{l}\text { April } \\
7, \\
65\end{array}$ & $\begin{array}{l}\text { Pacific } \\
\text { Ocean }\end{array}$ & & 7 & 2 & + & & & \\
\hline
\end{tabular}

Parasitic Animals:

Iots: 4 squid beaks

Notes on Squid:

fish

fish remains

eyebalis (6)

\section{Notes on Fish: 1. Head of one digested. \\ 2. Fish remains with bones.}

General Notes:

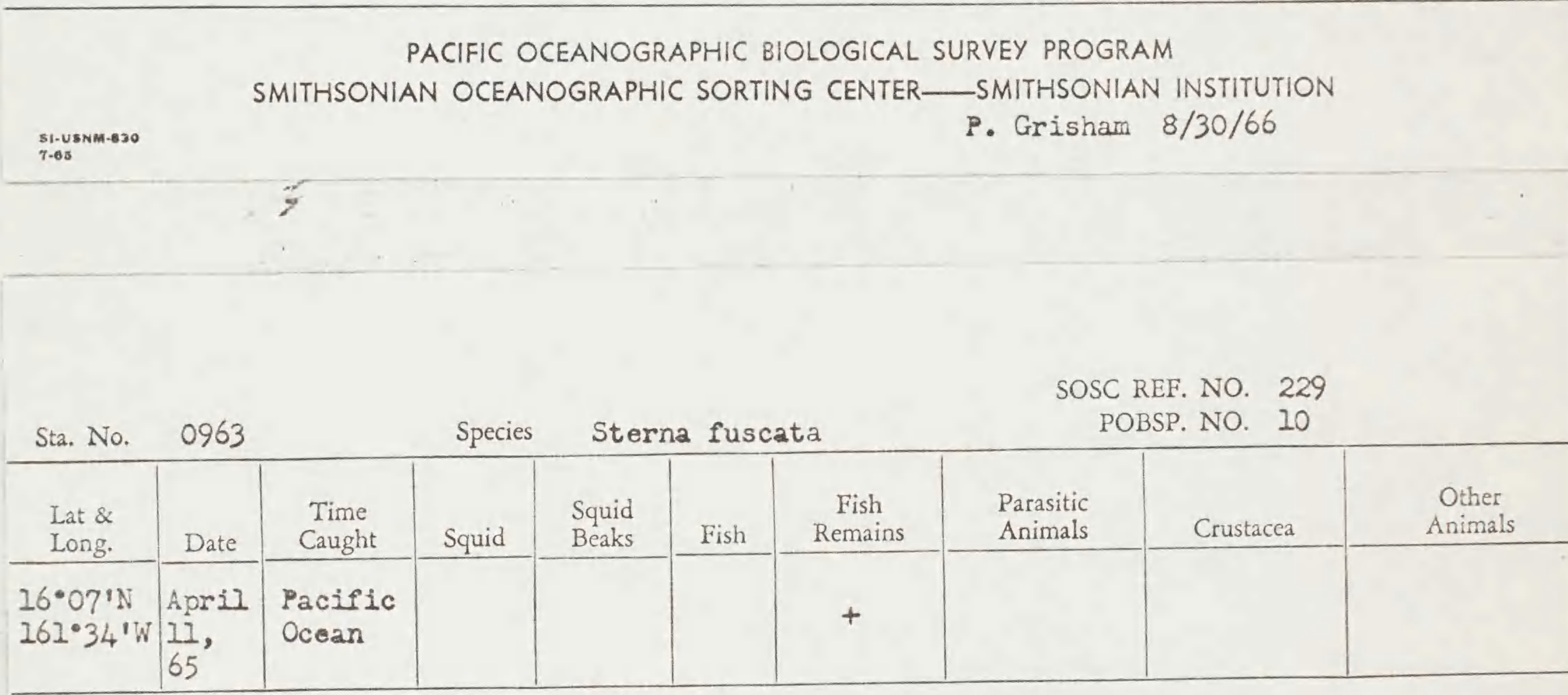

Parasitic Animals:

lots: 1 fish remains

Notes on Squid:

Notes on Fish:

Fish remains with bones.

General Notes:

PACIFIC OCEANOGRAPHIC BIOLOGICAL SURVEY PROGRAM

SMITHSONIAN OCEANOGRAPHIC SORTING CENTER—SMITHSONIAN INSTITUTION

S1.USNM-630

P. Grisham $8 / 4 / 66$ 


\begin{tabular}{|c|c|c|c|c|c|c|c|c|c|}
\hline Sta. No. & 0964 & & Specie: & Phae & $\mathrm{n} r v$ & lcauda & & $\begin{array}{l}\text { NO. } \\
\text { NO. }\end{array}$ & \\
\hline $\begin{array}{l}\text { Lat \& } \\
\text { Long. }\end{array}$ & Date & $\begin{array}{l}\text { Time } \\
\text { Caught }\end{array}$ & Squid & $\begin{array}{l}\text { Squid } \\
\text { Beaks }\end{array}$ & Fish & $\begin{array}{c}\text { Fish } \\
\text { Remains }\end{array}$ & $\begin{array}{l}\text { Parasitic } \\
\text { Animals }\end{array}$ & Crustacea & $\begin{array}{l}\text { Other } \\
\text { Animals }\end{array}$ \\
\hline $\begin{array}{r}26^{\circ} 08^{\prime} \mathrm{N} \\
261^{\circ} 50^{\circ} \mathrm{W}\end{array}$ & $\begin{array}{l}\text { Apri1 } \\
\frac{11}{65} \text {, }\end{array}$ & $\begin{array}{l}\text { Pacific } \\
\text { Ocean }\end{array}$ & & & 3 & + & & & \\
\hline
\end{tabular}

Parasitic Animals:

lots: 3 fish

Notes on Squid:

fish remains

eyeballs (1)

Notes on Fish:

Heads digested.

General Notes:

PACIFIC OCEANOGRAPHIC BIOLOGICAL SURVEY PROGRAM

SMITHSONIAN OCEANOGRAPHIC SORTING CENTER—SMITHSONIAN INSTITUTION

P. Grisham 8/16/66

\begin{tabular}{|c|c|c|c|c|c|c|c|c|c|}
\hline Sta. No. & 0965 & & Speci & & $12 \mathrm{f}$ & ata & & $\begin{array}{l}\text { No. } \\
\text { NO. }\end{array}$ & \\
\hline $\begin{array}{l}\text { Lat \& } \\
\text { Long. }\end{array}$ & Date & $\begin{array}{c}\text { Time } \\
\text { Caught }\end{array}$ & Squid & $\begin{array}{l}\text { Squid } \\
\text { Beaks }\end{array}$ & Fish & $\begin{array}{c}\text { Fish } \\
\text { Remains }\end{array}$ & $\begin{array}{l}\text { Parasitic } \\
\text { Animals }\end{array}$ & Crustacea & $\begin{array}{c}\text { Other } \\
\text { Animals }\end{array}$ \\
\hline $\begin{array}{l}16^{\circ} 37^{\prime} \mathrm{N} \\
169^{\circ} 04^{\prime} \mathrm{W}\end{array}$ & $\begin{array}{l}\text { Apri1 } \\
13 \\
65\end{array}$ & $\begin{array}{l}\text { Pacific } \\
\text { Ocean }\end{array}$ & & & & - & & & \\
\hline
\end{tabular}

Parasitic Animals:

Notes on Squid:

lots: I squid remains

Notes on Fish:

General Notes:

PACIFIC OCEANOGRAPHIC BIOLOGICAL SURVEY PROGRAM

SMITHSONIAN OCEANOGRAPHIC SORTING CENTER—SMITHSONIAN INSTITUTION

S1. USNM.830

P. Grisham 8/23/66 
SOSC REF. NO. 229

POBSP. NO. 10

\begin{tabular}{|c|c|c|c|c|c|c|c|c|c|}
\hline $\begin{array}{l}\text { Lat \& } \\
\text { Long. }\end{array}$ & & $\begin{array}{l}\text { Time } \\
\text { Caught }\end{array}$ & Squid & $\begin{array}{l}\text { Squid } \\
\text { Beaks }\end{array}$ & Fish & $\begin{array}{c}\text { Fish } \\
\text { Remains }\end{array}$ & $\begin{array}{l}\text { Parasitic } \\
\text { Animals }\end{array}$ & Crustacea & $\begin{array}{l}\text { Other } \\
\text { Animals }\end{array}$ \\
\hline $\begin{array}{l}25 \cdot 50: \mathrm{N} \\
269^{\circ} 26: \mathrm{W}\end{array}$ & $\begin{array}{l}\text { April } \\
13 \\
65\end{array}$ & $\begin{array}{l}\text { Pacific } \\
\text { acean }\end{array}$ & 2 & & 2 & + & & & \\
\hline
\end{tabular}

Parasitic Animals:

lots: 4 eyeba.1.2s (?)

fish remains

Notes on Squid:

Head of one digested.

fish

squids

Notes on Fish:

Good condition.

General Notes:

PACIFIC OCEANOGRAPHIC BIOLOGICAL SURVEY PROGRAM

SMITHSONIAN OCEANOGRAPHIC SORTING CENTER_-SMITHSONIAN ISTITUTION

51-USNM-830

7.68

z

Sta. No.

0967

Species

\begin{tabular}{|c|c|c|c|}
\hline $\begin{array}{l}\text { Lat \& } \\
\text { Long. }\end{array}$ & Date & $\begin{array}{l}\text { Time } \\
\text { Caught }\end{array}$ & Squid \\
\hline $\begin{array}{l}13 \cdot 30^{\circ} \mathrm{N} \\
169^{\circ} 38^{\prime} \mathrm{W}\end{array}$ & $\begin{array}{l}\text { Apria } \\
\frac{14}{65},\end{array}$ & $\begin{array}{l}\text { Pacific } \\
\text { Ocean }\end{array}$ & \\
\hline
\end{tabular}

Parasitic Animals:

Notes on Squid:

Notes on Fish:

Fish remains with bones.
Sula sula

SOSC REF. NO. 229

POBSP. NO. 10

\begin{tabular}{|c|c|c|c}
$\begin{array}{l}\text { Squid } \\
\text { Beaks }\end{array}$ & Fish & $\begin{array}{c}\text { Fish } \\
\text { Remains }\end{array}$ & $\begin{array}{c}\text { Parasitic } \\
\text { Animals }\end{array}$ \\
\hline 121 & + &
\end{tabular}

Crustacea

Other Animals lots: 4 squid remains squid beaks eyeballs (6) fish remains

\footnotetext{
General Notes:
}

PACIFIC OCEANOGRAPHIC BIOLOGICAL SURVEY PROGRAM 
SOSC REF. NO. 229

POBSP. NO. 10

\begin{tabular}{|c|c|c|c|c|c|c|c|c|c|}
\hline $\begin{array}{l}\text { Lat \& } \\
\text { Long. }\end{array}$ & Date & $\begin{array}{c}\text { Time } \\
\text { Caught }\end{array}$ & Squid & $\begin{array}{l}\text { Squid } \\
\text { Beaks }\end{array}$ & Fish & $\begin{array}{c}\text { Fish } \\
\text { Remains }\end{array}$ & $\begin{array}{l}\text { Parasitic } \\
\text { Animals }\end{array}$ & Crustacea & $\begin{array}{l}\text { Other } \\
\text { Animals }\end{array}$ \\
\hline $\begin{array}{l}13^{\circ} \mathrm{I}^{\prime} \mathrm{N} \\
169^{\circ} 58^{\prime} \mathrm{W}\end{array}$ & $\begin{array}{l}\text { April } \\
\frac{14}{65},\end{array}$ & $\begin{array}{l}\text { Pacific } \\
\text { Ocean }\end{array}$ & 2 & 100 & & + & & & \\
\hline
\end{tabular}

Parasitic Animals:

lots: 4 squid beaks

Notes on Squid:

Heads digested. squids

fish remains eyeballs (16)

Notes on Fish:

Fish remains with bones and half of a fish.

General Notes:

PACIFIC OCEANOGRAPHIC BIOLOGICAL SURVEY PROGRAM

SMITHSONIAN OCEANOGRAPHIC SORTING CENTER—SMITHSONIAN INSTITUTION

P. Grisham 8/15/66

SI.USNMA 830

7.65

$-5$

3

SOSC REF. NO.

POBSP. NO. 229

\begin{tabular}{|c|c|}
\hline $\begin{array}{l}\text { Lat \& } \\
\text { Long. }\end{array}$ & Date \\
\hline $\begin{array}{l}15^{\circ} 24^{\prime} \mathrm{N} \\
169^{\circ} 54^{\prime} \mathrm{W}\end{array}$ & $\begin{array}{l}\text { April } \\
16, \\
65\end{array}$ \\
\hline
\end{tabular}

Species

Sula dactylatra

POBSP. NO. 10

Parasitic Animals:

Notes on Squid:

lots: 2 fish

fish remains

\footnotetext{
Notes on Fish:

1. Fish remains with bones and scales.

2. Fair condition with heads attached.
}

General Notes:

PACIFIC OCEANOGRAPHIC BIOLOGICAL SURVEY PROGRAM

SMITHSONIAN OCEANOGRAPHIC SORTING CENTER—SMITHSONIAN INSTITUTION

P. Grisham 8/30/66 
SOSC REF. NO. 229

\begin{tabular}{|c|c|c|c|c|c|c|c|c|c|}
\hline $\begin{array}{l}\text { Lat \& } \\
\text { Long. }\end{array}$ & Date & $\begin{array}{l}\text { Time } \\
\text { Caught }\end{array}$ & Squid & $\begin{array}{l}\text { Squid } \\
\text { Beaks }\end{array}$ & Fish & $\begin{array}{c}\text { Fish } \\
\text { Remains }\end{array}$ & $\begin{array}{l}\text { Parasitic } \\
\text { Animals }\end{array}$ & Crustacea & $\begin{array}{l}\text { Other } \\
\text { Animals }\end{array}$ \\
\hline $\begin{array}{l}-5^{\circ} 30^{\prime} \mathrm{N} \\
-70^{\circ} 08^{\prime} \mathrm{W}\end{array}$ & $\begin{array}{l}\text { April } \\
65 \\
65\end{array}$ & $\begin{array}{l}\text { Pacific } \\
\text { Ocean }\end{array}$ & & 2 & & - & & & \\
\hline
\end{tabular}

Parasitic Animals:

Notes on Squid:

Iots: 2 squid beaks

digested remains

Notes on Fish:

General Notes:

PACIFIC OCEANOGRAPHIC BIOLOGICAL SURVEY PROGRAM SMITHSONIAN OCEANOGRAPHIC SORTING CENTER—SMITHSONIAN INSTITUTION

P. Grisham 8/9/66

S1.U5NM-830

7-63

3

SOSC REF. NO. 229

\begin{tabular}{|c|c|c|c|c|c|c|c|c|c|}
\hline $\begin{array}{l}\text { Lat \& } \\
\text { Long. }\end{array}$ & Date & $\begin{array}{l}\text { Time } \\
\text { Caught }\end{array}$ & Squid & $\begin{array}{l}\text { Squid } \\
\text { Beaks }\end{array}$ & Fish & $\begin{array}{c}\text { Fish } \\
\text { Remains }\end{array}$ & $\begin{array}{l}\text { Parasitic } \\
\text { Animals }\end{array}$ & Crustacea & $\begin{array}{c}\text { Other } \\
\text { Animals }\end{array}$ \\
\hline $\begin{array}{l}15^{\circ} 27^{\prime} \mathrm{N} \\
170^{\circ} 12^{\prime} \mathrm{W}\end{array}$ & $\begin{array}{l}\text { Apri1 } \\
16, \\
65\end{array}$ & $\begin{array}{l}\text { Pacific } \\
\text { Ocean }\end{array}$ & & & & - & & & \\
\hline
\end{tabular}

Parasitic Animals:

lots: 0

Notes on Squid:

Notes on Fish:

General Notes:

The stomach was empty.

PACIFIC OCEANOGRAPHIC BIOLOGICAL SURVEY PROGRAM

SMITHSONIAN OCEANOGRAPHIC SORTING CENTER-SMITHSONIAN INSTITUTION 


\begin{tabular}{|c|c|c|c|c|c|c|c|c|c|}
\hline Sta. No. & 0972 & & Speci & St & a $f u$ & ta & SO & $\begin{array}{l}\text { NO. } \\
\text { NO. }\end{array}$ & \\
\hline $\begin{array}{l}\text { Lat \& } \\
\text { Long. }\end{array}$ & Date & $\begin{array}{l}\text { Time } \\
\text { Caught }\end{array}$ & Squid & $\begin{array}{l}\text { Squid } \\
\text { Beaks }\end{array}$ & Fish & $\begin{array}{c}\text { Fish } \\
\text { Remains }\end{array}$ & $\begin{array}{l}\text { Parasitic } \\
\text { Animals }\end{array}$ & Crustacea & $\begin{array}{c}\text { Other } \\
\text { Animals }\end{array}$ \\
\hline $\begin{array}{l}15^{\circ} 24^{\prime} \mathrm{N} \\
170^{\circ} 16^{\prime} \mathrm{W}\end{array}$ & $\begin{array}{l}\text { April } \\
16, \\
65\end{array}$ & $\begin{array}{l}\text { Pacific } \\
\text { Ocean }\end{array}$ & & & & - & & & \\
\hline
\end{tabular}

Parasitic Animals:

Lots: 0

Notes on Squid:

Notes on Fish:

General Notes:

The stomach was empty.

PACIFIC OCEANOGRAPHIC BIOLOGICAL SURVEY PROGRAM

SMITHSONIAN OCEANOGRAPHIC SORTING CENTER_-SMITHSONIAN INSTITUTION

S1.USNM-830

P. Grisham 8/15/66

$\underset{7.63}{\operatorname{Sin} 0 \mathrm{~s}}$

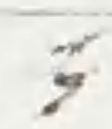

\begin{tabular}{|c|c|c|c|c|c|c|c|c|c|}
\hline Sta. No. & 09 & & Speci & Ste & fus & & & $\begin{array}{l}\text { NO. } \\
\text { NO. }\end{array}$ & \\
\hline $\begin{array}{l}\text { Lat \& } \\
\text { Long. }\end{array}$ & Date & $\begin{array}{c}\text { Time } \\
\text { Caught }\end{array}$ & Squid & $\begin{array}{l}\text { Squid } \\
\text { Beaks }\end{array}$ & Fish & $\begin{array}{c}\text { Fish } \\
\text { Remains }\end{array}$ & $\begin{array}{l}\text { Parasitic } \\
\text { Animals }\end{array}$ & Crustacea & $\begin{array}{c}\text { Other } \\
\text { Animals }\end{array}$ \\
\hline $\begin{array}{l}15^{\circ} 22^{\prime} \mathrm{N} \\
170^{\circ} 19^{\prime} \mathrm{W}\end{array}$ & $\begin{array}{l}\text { Apri1 } \\
16, \\
65\end{array}$ & $\begin{array}{l}\text { Pacific } \\
\text { Ocean }\end{array}$ & & & & - & & & \\
\hline
\end{tabular}

Parasitic Animals:

Notes on Squid:

Iots: 0

Notes on Fish:

General Notes:

The stomach was empty.

PACIFIC OCEANOGRAPHIC BIOLOGICAL SURVEY PROGRAM

SMITHSONIAN OCEANOGRAPHIC SORTING CENTER—SMITHSONIAN INSTITUTION 
SOSC REF. NO.

229

Sta. No.

\begin{tabular}{l|l|}
\hline $\begin{array}{l}\text { Lat \& } \\
\text { Long. }\end{array}$ & Date \\
\hline \begin{tabular}{l|l}
$15^{\circ} 10^{\prime} \mathrm{N}$ & Apri工 \\
$170^{\circ} 28^{\prime} \mathrm{W}$ & 16 \\
\hline
\end{tabular} \\
\hline
\end{tabular}

Parasitic Animals:

Notes on Squid:
POBSP. NO. 10

\begin{tabular}{|c|c|c|c|c|c|c|}
\hline Squid & $\begin{array}{l}\text { Squid } \\
\text { Beaks }\end{array}$ & Fish & $\begin{array}{c}\text { Fish } \\
\text { Remains }\end{array}$ & $\begin{array}{l}\text { Parasitic } \\
\text { Animals }\end{array}$ & Crustacea & $\begin{array}{l}\text { Other } \\
\text { Animals }\end{array}$ \\
\hline & 1 & & - & & & \\
\hline
\end{tabular}

lots: 2 squid beaks digested remains

\section{Notes on Fish:}

General Notes:

Digested remains beyond identification.

PACIFIC OCEANOGRAPHIC BIOLOGICAL SURVEY PROGRAM

SMITHSONIAN OCEANOGRAPHIC SORTING CENTER—SMITHSONIAN INSTITUTION

P. Grisham 7/27/66

SI-USNM-830

7-65

SOSC REF. NO. 229

\begin{tabular}{|c|c|c|c|c|c|c|c|c|c|}
\hline Sta. No. & 097 : & & Specie: & & na fi & ata & & NO. & \\
\hline $\begin{array}{l}\text { Lat \& } \\
\text { Long. }\end{array}$ & Date & $\begin{array}{l}\text { Time } \\
\text { Caught }\end{array}$ & Squid & $\begin{array}{l}\text { Squid } \\
\text { Beaks }\end{array}$ & Fish & $\begin{array}{c}\text { Fish } \\
\text { Remains }\end{array}$ & $\begin{array}{l}\text { Parasitic } \\
\text { Animals }\end{array}$ & Crustacea & $\begin{array}{l}\text { Other } \\
\text { Animals }\end{array}$ \\
\hline $\begin{array}{l}15^{\circ} 10^{\prime} \mathrm{N} \\
170^{\circ} 28^{\prime} \mathrm{W}\end{array}$ & $\begin{array}{l}\text { April } \\
16, \\
65\end{array}$ & $\begin{array}{l}\text { Pacific } \\
\text { Ocean }\end{array}$ & & 2 & & + & & & \\
\hline
\end{tabular}

Parasitic Animals:

lots: 2 fish remains squid beaks

Notes on Squid:

Notes on Fish:

Fish remains with bones.

General Notes:

PACIFIC OCEANOGRAPHIC BIOLOGICAL SURVEY PROGRAM SMITHSONIAN OCEANOGRAPHIC SORTING CENTER_SMITHSONIAN INSTITUTION 
SOSC REF. NO. 229

Sta. No. 0976 Species Sula sula

POBSP. NO. 10

\begin{tabular}{|c|c|c|c|c|c|c|c|c|c|}
\hline $\begin{array}{l}\text { Lat \& } \\
\text { Long. }\end{array}$ & Date & $\begin{array}{l}\text { Time } \\
\text { Caught }\end{array}$ & Squid & $\begin{array}{l}\text { Squid } \\
\text { Beaks }\end{array}$ & Fish & $\begin{array}{c}\text { Fish } \\
\text { Remains }\end{array}$ & $\begin{array}{l}\text { Parasitic } \\
\text { Animals }\end{array}$ & Crustacea & $\begin{array}{c}\text { Other } \\
\text { Animals }\end{array}$ \\
\hline $\begin{array}{l}25^{\circ} 10^{\prime} \mathrm{N} \\
170^{\circ} 31^{\prime} \mathrm{W}\end{array}$ & $\begin{array}{l}\text { Apri1 } \\
16, \\
65\end{array}$ & $\begin{array}{l}\text { Pácific } \\
\text { Ocean }\end{array}$ & & & 3 & + & & & \\
\hline
\end{tabular}

Parasitic Animals:

lots: 2 fish

Notes on Squid:

fish remains

\section{Notes on Fish: 1. Poor condition; heads digested. \\ 2. Fish remains with bones.}

General Notes:

PACIFIC OCEANOGRAPHIC BIOLOGICAL SURVEY PROGRAM SMITHSONIAN OCEANOGRAPHIC SORTING CENTER—SMITHSONIAN INSTITUTION

SOSC REF. NO. 229

\begin{tabular}{|c|c|c|c|c|c|c|c|c|c|}
\hline Sta. No. & 0977 & & Species & Ste & fus & & & NO. 10 & \\
\hline $\begin{array}{l}\text { Lat \& } \\
\text { Long. }\end{array}$ & Date & $\begin{array}{l}\text { Time } \\
\text { Caught }\end{array}$ & Squid & $\begin{array}{l}\text { Squid } \\
\text { Beaks }\end{array}$ & Fish & $\begin{array}{c}\text { Fish } \\
\text { Remains }\end{array}$ & $\begin{array}{l}\text { Parasitic } \\
\text { Animals }\end{array}$ & Crustacea & $\begin{array}{c}\text { Other } \\
\text { Animals }\end{array}$ \\
\hline $\begin{array}{l}14^{\circ} 59^{\prime} \mathrm{N} \\
170^{\circ} 37^{\prime} \mathrm{W}\end{array}$ & $\begin{array}{l}\text { Apri] } \\
16, \\
65\end{array}$ & $\begin{array}{l}\text { Pacific } \\
\text { Ocean }\end{array}$ & & & & + & & & \\
\hline
\end{tabular}

Parasitic Animals:

lots: 1 fish remains

Notes on Squid:

Notes on Fish:

Fish remains with bones.

General Notes:

PACIFIC OCEANOGRAPHIC BIOLOGICAL SURVEY PROGRAM SMITHSONIAN OCEANOGRAPHIC SORTING CENTER_-SMITHSONIAN INSTITUTION 
SOSC REF. NO.

229

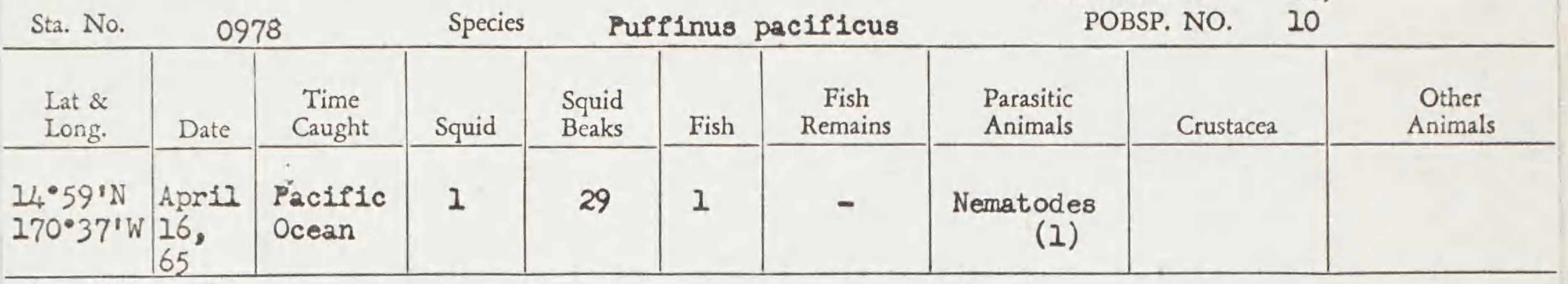

Parasitic Animals:

lots: 6 squid beaks

\section{Notes on Squid:}

Head digested. nematodes digested romains fish.

Notes on Fish:

Head digested and in poor condition.

squid

seeds

General Notes:

Digested remains with squid beak fragments.

PACIFIC OCEANOGRAPHIC BIOLOGICAL SURVEY PROGRAM SMITHSONIAN OCEANOGRAPHIC SORTING CENTER—SMITHSONIAN INSTITUTION

SI-USNM-830 P. Grisham $8 / 8 / 66$ 7-65 $\bar{y}$

\begin{tabular}{|c|c|c|}
\hline $\begin{array}{l}\text { Lat \& } \\
\text { Long. }\end{array}$ & Date & $\begin{array}{l}\text { Time } \\
\text { Caught }\end{array}$ \\
\hline $\begin{array}{l}14 \cdot 55^{\prime} \mathrm{N} \\
170^{\circ} 41^{\prime} \mathrm{W}\end{array}$ & $\begin{array}{l}\text { April } \\
16 \\
65\end{array}$ & $\begin{array}{l}\text { Pacific } \\
\text { Ocean }\end{array}$ \\
\hline
\end{tabular}

Parasitic Animals:

Notes on Squid:

Notes on Fish:

Fish remains with bones.
SOSC REF NO. 229 POBSP. NO. 10

\begin{tabular}{|c|c|c|c|} 
Species & \multicolumn{2}{c}{ Sterna fuscata } \\
Squid & $\begin{array}{c}\text { Squid } \\
\text { Beaks }\end{array}$ & Fish & $\begin{array}{c}\text { Fish } \\
\text { Remains }\end{array}$ \\
\hline & &
\end{tabular}

\begin{tabular}{|c|c|c}
$\begin{array}{c}\text { Parasitic } \\
\text { Animals }\end{array}$ & Crustacea & $\begin{array}{c}\text { Other } \\
\text { Animals }\end{array}$ \\
\hline &
\end{tabular}

lots: 1 fish remains

General Notes: 
SOSC REF. NO. 229

\begin{tabular}{|c|c|c|c|c|c|c|c|c|c|}
\hline Sta. No. & 0980 & & Speci & Pha & on 1 & urus & & NO. I & \\
\hline $\begin{array}{l}\text { Lat \& } \\
\text { Long. }\end{array}$ & Date & $\begin{array}{c}\text { Time } \\
\text { Caught }\end{array}$ & Squid & $\begin{array}{l}\text { Squid } \\
\text { Beaks }\end{array}$ & Fish & $\begin{array}{c}\text { Fish } \\
\text { Remains }\end{array}$ & $\begin{array}{l}\text { Parasitic } \\
\text { Animals }\end{array}$ & Crustacea & $\begin{array}{c}\text { Other } \\
\text { Animals }\end{array}$ \\
\hline $\begin{array}{l}144^{\circ} 47^{\prime} \mathrm{N} \\
170^{\circ} 50^{\prime} \mathrm{W}\end{array}$ & $\begin{array}{l}\text { April } \\
16, \\
65\end{array}$ & $\begin{array}{l}\text { Pacific } \\
\text { Ocean }\end{array}$ & & 2 & & $\begin{array}{lll}+ & & -1\end{array}$ & & & \\
\hline
\end{tabular}

Parasitic Animals:

lots: 2 fish remains

Notes on Squid:

squid beaks

Notes on Fish:

Fish remains with bones.

General Notes:

PACIFIC OCEANOGRAPHIC BIOLOGICAL SURVEY PROGRAM

SMITHSONIAN OCEANOGRAPHIC SORTING CENTER — SMITHSONIAN INSTITUTION

$51-U S N M-830$

P. Grisham 8/9/66

7.65

\begin{tabular}{|c|c|c|c|c|c|c|c|c|c|}
\hline Sta. No. & $098]$ & & Speci & St & a fo & & & $\begin{array}{l}\text { NO. } \\
\text { NO. }\end{array}$ & \\
\hline $\begin{array}{l}\text { Lat \& } \\
\text { Long. }\end{array}$ & Date & $\begin{array}{l}\text { Time } \\
\text { Caught }\end{array}$ & Squid & $\begin{array}{l}\text { Squid } \\
\text { Beaks }\end{array}$ & Fish & $\begin{array}{c}\text { Fish } \\
\text { Remains }\end{array}$ & $\begin{array}{l}\text { Parasitic } \\
\text { Animals }\end{array}$ & Crustacea & $\begin{array}{l}\text { Other } \\
\text { Animals }\end{array}$ \\
\hline$\frac{14}{172} \cdot 36: 03 \mathrm{~N}$ & $\begin{array}{l}\text { Apri工 } \\
16, \\
65\end{array}$ & $\begin{array}{l}\text { Pacific } \\
\text { Ocean }\end{array}$ & 3 & & 1 & + & & & \\
\hline
\end{tabular}

Parasitic Animals:

Notes on Squid:

1. Head of one digested.

2. Tentacles of one detached.

lots: 3 fish remains

fish (eel)

squids

Notes on Fish:

1. Head digested.

General Notes:

PACIFIC OCEANOGRAPHIC BIOLOGICAL SURVEY PROGRAM

SMITHSONIAN OCEANOGRAPHIC SORTING CENTER—SMITHSONIAN INSTITUTION

P. Grisham

$8 / 24_{4} / 66$ 
SOSC REF. NO. 229

\begin{tabular}{|c|c|c|c|c|c|c|c|c|c|}
\hline Sta. No. & 098: & & Speci & & na $\mathrm{f}$ & ata & & NO. 10 & \\
\hline $\begin{array}{l}\text { Lat \& } \\
\text { Long. }\end{array}$ & Date & $\begin{array}{l}\text { Time } \\
\text { Caught }\end{array}$ & Squid & $\begin{array}{l}\text { Squid } \\
\text { Beaks }\end{array}$ & Fish & $\begin{array}{c}\text { Fish } \\
\text { Remains }\end{array}$ & $\begin{array}{l}\text { Parasitic } \\
\text { Animals }\end{array}$ & Crustacea & $\begin{array}{l}\text { Other } \\
\text { Animals }\end{array}$ \\
\hline $\begin{array}{l}14^{\circ} 36^{\prime} \mathrm{N} \\
171^{\circ} 03^{\prime} \mathrm{W}\end{array}$ & $\begin{array}{l}\text { April } \\
16, \\
65\end{array}$ & $\begin{array}{l}\text { Pacific } \\
\text { Ocean }\end{array}$ & 2 & & & - & & 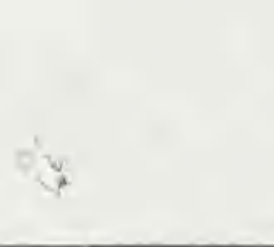 & \\
\hline
\end{tabular}

Parasitic Animals:

lots: 2 dicested remains

$\begin{array}{ll}\text { Notes on Squid: } & \text { 1. Head of one digested. } \\ & \text { 2. Head of second detached. }\end{array}$

Notes on Fish:

General Notes:

1. Digested remains with pieces of squid.

PACIFIC OCEANOGRAPHIC BIOLOGICAL SURVEY PROGRAM

SMITHSONIAN OCEANOGRAPHIC SORTING CENTER—SMITHSONIAN INSTITUTION

51-USNM-\$30

7.65

P. Grisham 8/24/66

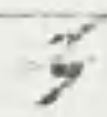

SOSC REF. NO. 229

Sta. No. 0983

Species Sterna fuscata

POBSP. NO. 10

\begin{tabular}{|c|c|c|c|c|c|c|c|c|c|}
\hline $\begin{array}{l}\text { Lat \& } \\
\text { Long. }\end{array}$ & Date & $\begin{array}{l}\text { Time } \\
\text { Caught }\end{array}$ & Squid & $\begin{array}{l}\text { Squid } \\
\text { Beaks }\end{array}$ & Fish & $\begin{array}{c}\text { Fish } \\
\text { Remains }\end{array}$ & $\begin{array}{l}\text { Parasitic } \\
\text { Animals }\end{array}$ & Crustacea & $\begin{array}{c}\text { Other } \\
\text { Animals }\end{array}$ \\
\hline $\begin{array}{l}\mathrm{I}_{4} \cdot 36^{\prime} \mathrm{N} \\
171^{\circ} 03^{\prime} \mathrm{W}\end{array}$ & $\begin{array}{l}\text { April } \\
16,\end{array}$ & $\begin{array}{l}\text { Pacific } \\
\text { Ocean }\end{array}$ & 2 & & & + & & & \\
\hline
\end{tabular}

Parasitic Animals:

lots: 2 squids

Notes on Squid:

Head of one digested.

Notes on Fish: Fish remains with bones and half of a fish.

General Notes:

PACIFIC OCEANOGRAPHIC BIOLOGICAL SURVEY PROGRAM SMITHSONIAN OCEANOGRAPHIC SORTING CENTER—SMITHSONIAN INSTITUTION 
SOSC REF. NO. 229

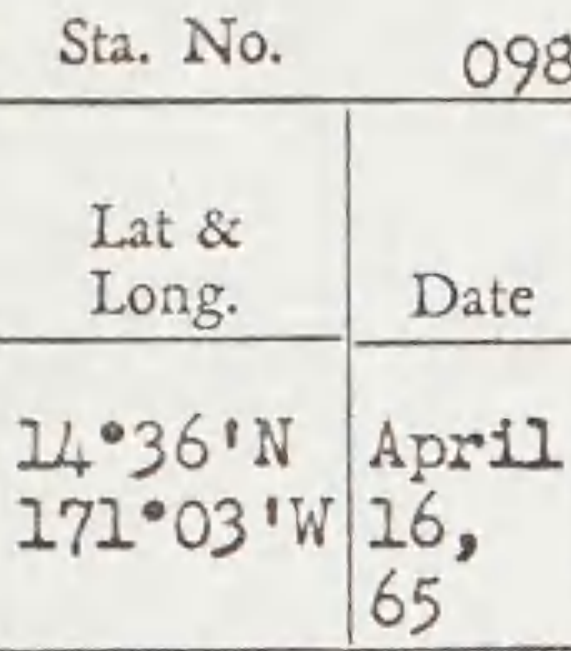

Sterna fuscata

POBSP. NO. 10

Parasitic Animals:

lots: 3 fish remains

Notes on Squid: squid remains eyeballs (I)

\section{Notes on Fish:}

Fish remains with bones.

General Notes:

PACIFIC OCEANOGRAPHIC BIOLOGICAL SURVEY PROGRAM SMITHSONIAN OCEANOGRAPHIC SORTING CENTER—SMITHSONIAN INSTITUTION

\begin{tabular}{|c|c|c|c|c|c|c|c|c|c|}
\hline Sta. No. & 098: & & Speci & Ste & fu & & & $\begin{array}{l}\text { NO. } \\
\text { NO. }\end{array}$ & \\
\hline $\begin{array}{l}\text { Lat \& } \\
\text { Long. }\end{array}$ & Date & $\begin{array}{l}\text { Time } \\
\text { Caught }\end{array}$ & Squid & $\begin{array}{l}\text { Squid } \\
\text { Beaks }\end{array}$ & Fish & $\begin{array}{c}\text { Fish } \\
\text { Remains }\end{array}$ & $\begin{array}{l}\text { Parasitic } \\
\text { Animals }\end{array}$ & Crustacea & $\begin{array}{c}\text { Other } \\
\text { Animals }\end{array}$ \\
\hline $\begin{array}{l}14 \cdot 361 \mathrm{~N} \\
171 \cdot 031 \mathrm{~W}\end{array}$ & $\begin{array}{l}\text { April } \\
16, \\
65\end{array}$ & $\begin{array}{l}\text { Pacific } \\
\text { Ocean }\end{array}$ & & & & + & & & \\
\hline
\end{tabular}

Parasitic Animals:

Notes on Squid:

lots: 1 fish remains

Notes on Fish:

Fish remains with bones.

General Notes: 
SOSC REF. NO. 229

Sta. No. 0986 Species Sterna fuscata POBSP. NO.

10

\begin{tabular}{|c|c|c|c|c|c|c|c|c|c|}
\hline $\begin{array}{l}\text { Lat \& } \\
\text { Long. }\end{array}$ & Date & $\begin{array}{l}\text { Time } \\
\text { Caught }\end{array}$ & Squid & $\begin{array}{l}\text { Squid } \\
\text { Beaks }\end{array}$ & Fish & $\begin{array}{c}\text { Fish } \\
\text { Remains }\end{array}$ & $\begin{array}{l}\text { Parasitic } \\
\text { Animals }\end{array}$ & Crustacea & $\begin{array}{c}\text { Other } \\
\text { Animals }\end{array}$ \\
\hline $\begin{array}{l}\mathrm{U}_{4} \cdot 36 \mathrm{~N} \\
172 \cdot 03 \cdot \mathrm{W}\end{array}$ & $\begin{array}{l}\text { Apri1 } \\
16, \\
65\end{array}$ & $\begin{array}{l}\text { Pacific } \\
\text { Ocean }\end{array}$ & & & & - & & & \\
\hline
\end{tabular}

Parasitic Animals:

lots: 1 digested remains

Notes on Squid:

Notes on Fish:

General Notes:

Digested remains beyond identification.

PACIFIC OCEANOGRAPHIC BIOLOGICAL SURVEY PROGRAM

SMITHSONIAN OCEANOGRAPHIC SORTING CENTER—SMITHSONIAN INSTITUTION

ST-USNM-830

P. Grisham 8/5/66

T-6s

SOSC REF. NO. 229

POBSP. NO. 10

\begin{tabular}{|c|c|c|c|c|c|c|c|c|c|}
\hline & $098 ?$ & & Species & Phae & on $l e$ & urus & & 19u. 10 & \\
\hline $\begin{array}{l}\text { Lat \& } \\
\text { Long. }\end{array}$ & Date & $\begin{array}{l}\text { Time } \\
\text { Caught }\end{array}$ & Squid & $\begin{array}{l}\text { Squid } \\
\text { Beaks }\end{array}$ & Fish & $\begin{array}{c}\text { Fish } \\
\text { Remains }\end{array}$ & $\begin{array}{l}\text { Parasitic } \\
\text { Animals }\end{array}$ & Crustacea & $\begin{array}{l}\text { Other } \\
\text { Animals }\end{array}$ \\
\hline $\begin{array}{l}13 \cdot 40^{\prime} \mathrm{N} \\
172 \cdot 49^{\prime} \mathrm{W}\end{array}$ & $\begin{array}{l}\text { Apri1 } \\
17, \\
65\end{array}$ & $\begin{array}{l}\text { Pacific } \\
\text { Ocean }\end{array}$ & & 25 & & - & & & \\
\hline
\end{tabular}

Parasitic Animals:

Iots: 3 eyeballs (3) squid beaks

Notes on Squid: squid remains

Notes on Fish:

General Notes:

PACIFIC OCEANOGRAPHIC BIOLOGICAL SURVEY PROGRAM SMITHSONIAN OCEANOGRAPHIC SORTING CENTER—SMITHSONIAN INSTITUTION 
SOSC REF. NO. 229

\begin{tabular}{|c|c|c|c|c|c|c|c|c|c|}
\hline Sta. No. & 098 & & Species & Phaet & & cauda & & NO. 10 & \\
\hline $\begin{array}{l}\text { Lat \& } \\
\text { Long. }\end{array}$ & Date & $\begin{array}{c}\text { Time } \\
\text { Caught }\end{array}$ & Squid & $\begin{array}{l}\text { Squid } \\
\text { Beaks }\end{array}$ & Fish & $\begin{array}{c}\text { Fish } \\
\text { Remains }\end{array}$ & $\begin{array}{l}\text { Parasitic } \\
\text { Animals }\end{array}$ & Crustacea & $\begin{array}{c}\text { Other } \\
\text { Animals }\end{array}$ \\
\hline $\begin{array}{l}13^{\circ} 40^{\prime} \mathrm{N} \\
172 \cdot 49^{\prime} \mathrm{W}\end{array}$ & $\begin{array}{l}\text { April } \\
17, \\
65\end{array}$ & $\begin{array}{l}\text { Pacific } \\
\text { Ocean }\end{array}$ & & 19 & & - & & & \\
\hline
\end{tabular}

Parasitic Animals:

lots: 3 squid beaks

Notes on Squid:

eyeballs (5)

digested remains

Notes on Fish:

General Notes:

Digested remains beyond identification.

\begin{tabular}{|c|c|c|c|c|c|c|c|c|c|}
\hline $\begin{array}{l}\text { S1.USNM-830 } \\
7 \rightarrow-65\end{array}$ & \multicolumn{8}{|c|}{$\begin{array}{l}\text { PACIFIC OCEANOGRAPHIC BIOLOGICAL SURVEY PROGRAM } \\
\text { MITHSONIAN OCEANOGRAPHIC SORTING CENTER-SMITHSONIAN INSTITUTION } \\
\text { P. Grisham } 8 / 23 / 66\end{array}$} & \\
\hline & & $y$ & & & & & & & \\
\hline & & & & & & & & & \\
\hline Sta. No. & 0989 & & Specie & Sul & & $\operatorname{tr} 2$ & SOS & $\begin{array}{ll}\text { F. NO. } & 229 \\
\text { P. NO. } & 10\end{array}$ & \\
\hline $\begin{array}{l}\text { Lat \& } \\
\text { Long. }\end{array}$ & Date & $\begin{array}{l}\text { Time } \\
\text { Caught }\end{array}$ & Squid & $\begin{array}{l}\text { Squid } \\
\text { Beaks }\end{array}$ & Fish & $\begin{array}{c}\text { Fish } \\
\text { Remains }\end{array}$ & $\begin{array}{l}\text { Parasitic } \\
\text { Animals }\end{array}$ & Crustacea & $\begin{array}{c}\text { Other } \\
\text { Animals }\end{array}$ \\
\hline $\begin{array}{l}\mathrm{u}_{4} \cdot \mathrm{u}_{4} \mathrm{~N} \\
172^{\circ} \mathrm{H}^{\prime} \mathrm{W}\end{array}$ & $\begin{array}{l}\text { April } \\
17, \\
65\end{array}$ & $\begin{array}{l}\text { Pacific } \\
\text { Ocean }\end{array}$ & & 2 & 2 & + & & & \\
\hline
\end{tabular}

Parasitic Animals:

Notes on Squid:

lots: 3 fish

fish remains

squid beaks

$\begin{array}{ll}\text { Notes on Fish: } & \text { 1. Heads digested. } \\ & \text { 2. Fish remains with bones. }\end{array}$

General Notes:

PACIFIC OCEANOGRAPHIC BIOLOGICAL SURVEY PROGRAM SMITHSONIAN OCEANOGRAPHIC SORTING CENTER—SMITHSONIAN INSTITUTION

P. Grisham 8/30/66 
SOSC REF. NO. 229

\begin{tabular}{|c|c|c|c|c|c|c|c|c|c|}
\hline Sta. No. & 0990 & & Species & & thon & bricauda & & NO. & \\
\hline $\begin{array}{l}\text { Lat \& } \\
\text { Long. }\end{array}$ & Date & $\begin{array}{l}\text { Time } \\
\text { Caught }\end{array}$ & Squid & $\begin{array}{l}\text { Squid } \\
\text { Beaks }\end{array}$ & Fish & $\begin{array}{c}\text { Fish } \\
\text { Remains }\end{array}$ & $\begin{array}{l}\text { Parasitic } \\
\text { Animals }\end{array}$ & Crustacea & $\begin{array}{c}\text { Other } \\
\text { Animals }\end{array}$ \\
\hline $\begin{array}{l}1_{4} \cdot 26^{\prime} \mathrm{N} \\
172^{\bullet} 32^{\prime} \mathrm{W}\end{array}$ & $\begin{array}{l}\text { April } \\
27, \\
65\end{array}$ & $\begin{array}{l}\text { Pacific } \\
\text { Ocean }\end{array}$ & & 4 & & + & & & \\
\hline
\end{tabular}

Parasitic Animals:

lots: 3 digested remains

Notes on Squid: squid beaks

fish remains

\section{Notes on Fish:}

Fish remains with bones and half of a fish.

\section{General Notes:}

\section{PACIFIC OCEANOGRAPHIC BIOLOGICAL SURVEY PROGRAM} SMITHSONIAN OCEANOGRAPHIC SORTING CENTER—SMITHSONIAN INSTITUTION

SOSC REF. NO. 229

POBSP. NO. 10

\begin{tabular}{|c|c|c|c|c|c|c|c|c|c|}
\hline Sta. No. & 0991 & & Species & Ocear & roma & leucorhoa & & NO. 10 & \\
\hline $\begin{array}{l}\text { Lat \& } \\
\text { Long. }\end{array}$ & Date & $\begin{array}{l}\text { Time } \\
\text { Caught }\end{array}$ & Squid & $\begin{array}{l}\text { Squid } \\
\text { Beaks }\end{array}$ & Fish & $\begin{array}{c}\text { Fish } \\
\text { Remains }\end{array}$ & $\begin{array}{l}\text { Parasitic } \\
\text { Animals }\end{array}$ & Crustacea & $\begin{array}{l}\text { Other } \\
\text { Animals }\end{array}$ \\
\hline $\begin{array}{l}\frac{14}{172} 32^{\prime} \mathrm{N} \\
4^{\prime} \mathrm{W}\end{array}$ & $\begin{array}{l}\text { April } \\
17, \\
65\end{array}$ & $\begin{array}{l}\text { Pacific } \\
\text { Ocean }\end{array}$ & & & & - & & & \\
\hline
\end{tabular}

Parasitic Animals:

lots: 1 digested remains

Notes on Squid:

Notes on Fish:

General Notes:

Digested remains beyond identification.

PACIFIC OCEANOGRAPHIC BIOLOGICAL SURVEY PROGRAM SMITHSONIAN OCEANOGRAPHIC SORTING CENTER—SMITHSONIAN INSTITUTION 


\begin{tabular}{l|l|l|l|l|l|l|l|l|l} 
Sta. No. 0992 & \multicolumn{1}{c}{ Species } & \multicolumn{2}{c}{ Sula dactylatra } & \multicolumn{2}{c}{$\begin{array}{c}\text { OSC REF. NO. } \\
\text { POBSP. NO. }\end{array}$} & $\begin{array}{l}229 \\
10\end{array}$ \\
\hline $\begin{array}{l}\text { Lat \& } \\
\text { Long. }\end{array}$ & Date & $\begin{array}{l}\text { Time } \\
\text { Caught }\end{array}$ & Squid & $\begin{array}{l}\text { Squid } \\
\text { Beaks }\end{array}$ & Fish & $\begin{array}{c}\text { Fish } \\
\text { Remains }\end{array}$ & $\begin{array}{c}\text { Parasitic } \\
\text { Animals }\end{array}$ & Crustacea & $\begin{array}{c}\text { Other } \\
\text { Animals }\end{array}$ \\
\hline $\begin{array}{l}16 \cdot 28^{\prime} \mathrm{N} \\
171^{\circ} 10^{\prime} \mathrm{W}\end{array}$ & $\begin{array}{l}\text { April } \\
18, \\
65\end{array}$ & $\begin{array}{l}\text { Pacific } \\
\text { Ocean }\end{array}$ & & 12 & & + & $\begin{array}{c}\text { Nematode } \\
\text { (1) }\end{array}$ & & \\
\hline
\end{tabular}

Parasitic Animals:

lots: 4 fish remains

Notes on Squid:

squid beaks

nematode

eyeballs (3)

Notes on Fish: Fish remains with a fish broken in half.

General Notes:

PACIFIC OCEANOGRAPHIC BIOLOGICAL SURVEY PROGRAM

SMITHSONIAN OCEANOGRAPHIC SORTING CENTER — SMITHSONIAN INSTITUTION

$51-U 5 M M-830$
$7-65$

P. Grisham $8 / 30 / 66$

T-6s

Y

\begin{tabular}{|c|c|c|c|c|c|c|c|c|c|}
\hline $\begin{array}{l}\text { Lat \& } \\
\text { Long. }\end{array}$ & Date & $\begin{array}{l}\text { Time } \\
\text { Caught }\end{array}$ & Squid & $\begin{array}{l}\text { Squid } \\
\text { Beaks }\end{array}$ & Fish & $\begin{array}{c}\text { Fish } \\
\text { Remains }\end{array}$ & $\begin{array}{l}\text { Parasitic } \\
\text { Animals }\end{array}$ & Crustacea & $\begin{array}{l}\text { Other } \\
\text { Animals }\end{array}$ \\
\hline $\begin{array}{l}16 \cdot 36^{\prime} \mathrm{N} \\
172 \cdot 26^{\prime} \mathrm{W}\end{array}$ & $\begin{array}{l}\text { April } \\
18, \\
65\end{array}$ & $\begin{array}{l}\text { Pacific } \\
\text { Ocean }\end{array}$ & & & & - & & & \\
\hline
\end{tabular}

Parasitic Animals:

lots: I digested remains

Notes on Squid:

Notes on Fish:

General Notes:

Digested remains beyond identification.

PACIFIC OCEANOGRAPHIC BIOLOGICAL SURVEY PROGRAM

SMITHSONIAN OCEANOGRAPHIC SORTING CENTER — SMITHSONIAN INSTITUTION

S1-USNM-e30

P. Grisham 8/5/66 
SOSC REF. NO. 229

\begin{tabular}{l|l} 
Sta. No.0994 \\
\begin{tabular}{l|l} 
Lat \& \\
Long.
\end{tabular} & Date \\
\hline $\begin{array}{ll}16^{\circ} 36^{\prime} \mathrm{N} & \text { April } \\
171^{\circ} 26^{\prime} \mathrm{W} & 18, \\
65\end{array}$
\end{tabular}

Species Sterna fuscata

POBSP. NO. 10

Parasitic Animals:

Lots: 3 squid

fish remains

Notes on Squid:

Head attached.

Other

Animals

Pacific

Ocean

1

digested remains

Notes on Fish:

General Notes:

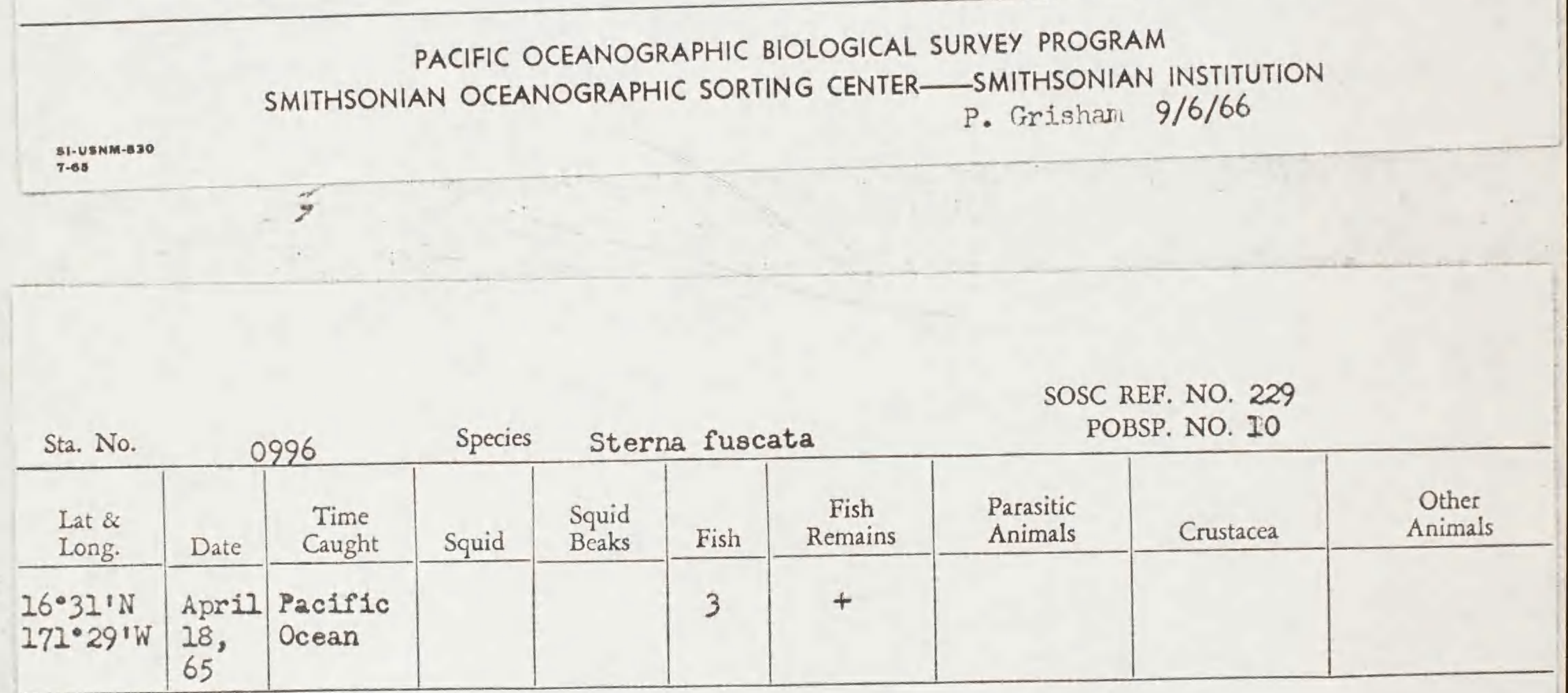

Parasitic Animals:

lots: 2 fish

Notes on Squid:

fish remains

Notes on Fish: 1. Heads of two digested.

2. Fish remains with bones.

General Notes:

PACIFIC OCEANOGRAPHIC BIOLOGICAL SURVEY PROGRAM

SMITHSONIAN OCEANOGRAPHIC SORTING CENTER—SMITHSONIAN INSTITUTION

$\operatorname{lin}_{7-65}^{51.4 N M-830}$

P. Grisham 8/30/66 
SOSC REF. NO. 229

\begin{tabular}{|c|c|c|c|c|c|c|c|c|c|}
\hline $\begin{array}{l}\text { Lat \& } \\
\text { Long. }\end{array}$ & Date & $\begin{array}{c}\text { Time } \\
\text { Caught }\end{array}$ & Squid & $\begin{array}{l}\text { Squid } \\
\text { Beaks }\end{array}$ & Fish & $\begin{array}{c}\text { Fish } \\
\text { Remains }\end{array}$ & $\begin{array}{l}\text { Parasitic } \\
\text { Animals }\end{array}$ & Crustacea & $\begin{array}{l}\text { Other } \\
\text { Animals }\end{array}$ \\
\hline $\begin{array}{l}16^{\circ} 36^{\prime} \mathrm{N} \\
171^{\circ} 26^{\prime} \mathrm{W}\end{array}$ & $\begin{array}{l}\text { April } \\
18, \\
65\end{array}$ & $\begin{array}{l}\text { Pacific } \\
\text { Ocean }\end{array}$ & 1 & & & + & & & \\
\hline
\end{tabular}

Parasitic Animals:

lots: 2 squid

Notes on Squid:

Head attached; good condition.

fish remains

\begin{tabular}{|c|c|}
\hline Notes on Fish: & Fish remains with pieces of $\mathrm{fish}$ \\
\hline General Notes: & 1 \\
\hline$\underset{7-68}{\text { S1-USNMA-830 }}$ & $\begin{array}{c}\text { PACIFIC OCEANOGRAPHIC BIOLOGICAL SURVEY PROGRAM } \\
\text { SMITHSONIAN OCEANOGRAPHIC SORTING CENTER-SMITHSONIAN INSTITUTION } \\
\text { P. Grisham } 8 / 31 / 66\end{array}$ \\
\hline
\end{tabular}

\begin{tabular}{|c|c|c|c|c|c|c|c|c|c|}
\hline \multirow{2}{*}{$\begin{array}{l}\text { Sta. No. } \\
\text { Lat \& } \\
\text { Long. }\end{array}$} & \multicolumn{2}{|l|}{0997} & Species & \multicolumn{3}{|c|}{ Oceanodroma leucorhoa } & \multicolumn{2}{|c|}{$\begin{array}{l}\text { SOSC REF. NO. } 229 \\
\text { POBSP. NO. } 10\end{array}$} & \\
\hline & Date & $\begin{array}{l}\text { Time } \\
\text { Caught }\end{array}$ & Squid & $\begin{array}{l}\text { Squid } \\
\text { Beaks }\end{array}$ & Fish & $\begin{array}{c}\text { Fish } \\
\text { Remains }\end{array}$ & $\begin{array}{l}\text { Parasitic } \\
\text { Animals } \\
\end{array}$ & Crustacea & $\begin{array}{l}\text { Other } \\
\text { Animals }\end{array}$ \\
\hline $\begin{array}{l}15^{\circ} 58^{\prime} \mathrm{N} \\
172^{\circ} \mathrm{O} 4^{\prime} \mathrm{W}\end{array}$ & $\begin{array}{l}\text { April } \\
18, \\
65\end{array}$ & $\begin{array}{l}\text { Pacific } \\
\text { Ocean }\end{array}$ & & 1 & & - & & & \\
\hline
\end{tabular}

Parasitic Animals:

lots: 3 squid beaks

Notes on Squid: ejeballs (4) digested remains

Notes on Fish:

General Notes:

Digested remains with seeds.

PACIFIC OCEANOGRAPHIC BIOLOGICAL SURVEY PROGRAM SMITHSONIAN OCEANOGRAPHIC SORTING CENTER_-SMITHSONIAN INSTITUTION 
SOSC REF. NO.

\begin{tabular}{|c|c|c|c|c|c|c|c|c|c|}
\hline $\begin{array}{l}\text { Lat \& } \\
\text { Long. }\end{array}$ & Date & $\begin{array}{l}\text { Time } \\
\text { Caught }\end{array}$ & Squid & $\begin{array}{l}\text { Squid } \\
\text { Beaks }\end{array}$ & Fish & $\begin{array}{c}\text { Fish } \\
\text { Remains }\end{array}$ & $\begin{array}{l}\text { Parasitic } \\
\text { Animals }\end{array}$ & Crustacea & $\begin{array}{c}\text { Other } \\
\text { Animals }\end{array}$ \\
\hline $\begin{array}{l}15^{\circ} 44^{\prime} \mathrm{N} \\
172^{\circ} 20^{\prime} \mathrm{W}\end{array}$ & $\begin{array}{l}\text { April } \\
18, \\
65\end{array}$ & $\begin{array}{l}\text { Pacilic } \\
\text { Ocean }\end{array}$ & 1 & 1 & & $\begin{array}{lll}+ & & \end{array}$ & & & \\
\hline
\end{tabular}

Parasitic Animals:

Lots: 4 squid
Notes on Squid:
1. Tail of squid digested. squid beaks
2. Squid remains with half of a squid and tentacles. squid remains fish remains

Notes on Fish:

1. Fish remains with bones.

General Notes:

PACIFIC OCEANOGRAPHIC BIOLOGICAL SURVEY PROGRAM SMITHSONIAN OCEANOGRAPHIC SORTING CENTER—SMITHSONIAN INSTITUTION

Si.USNM-e30

P. Grisham 8/8/66

7.65

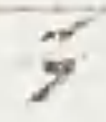

Sta. No. 0999

Species Sterna fuscata

SOSC REF. NO. 229

\begin{tabular}{|c|c|c|c|c|c|c|c|c|c|}
\hline $\begin{array}{l}\text { Lat \& } \\
\text { Long. }\end{array}$ & Date & $\begin{array}{l}\text { Time } \\
\text { Caught }\end{array}$ & Squid & $\begin{array}{l}\text { Squid } \\
\text { Beaks }\end{array}$ & Fish & $\begin{array}{c}\text { Fish } \\
\text { Remains }\end{array}$ & $\begin{array}{l}\text { Parasitic } \\
\text { Animals }\end{array}$ & Crustacea & $\begin{array}{c}\text { Other } \\
\text { Animals }\end{array}$ \\
\hline $\begin{array}{l}15^{\circ} 44_{4} \mathrm{~N} \\
172^{\circ} 20^{\prime} \mathrm{W}\end{array}$ & $\begin{array}{l}\text { April } \\
18, \\
65\end{array}$ & $\begin{array}{l}\text { Pacific } \\
\text { Ocean }\end{array}$ & & & & + & & & \\
\hline
\end{tabular}

Parasitic Animals:

lots: I fish remains

Notes on Squid:

Notes on Fish:

Fish remains with half of a fish.

General Notes:

PACIFIC OCEANOGRAPHIC BIOLOGICAL SURVEY PROGRAM

SMITHSONIAN OCEANOGRAPHIC SORTING CENTER—SMITHSONIAN INSTITUTION

P. Grisham 8/16/66 
SOSC REF. NO.

229

Sta. No. 1000

Species Sterna fuscata

POBSP. NO. 10

\begin{tabular}{c|c|c|c|c|c|c|c|c|c}
$\begin{array}{c}\text { Lat \& } \\
\text { Long. }\end{array}$ & Date & $\begin{array}{c}\text { Time } \\
\text { Caught }\end{array}$ & Squid & $\begin{array}{c}\text { Squid } \\
\text { Beaks }\end{array}$ & Fish & $\begin{array}{c}\text { Fish } \\
\text { Remains }\end{array}$ & $\begin{array}{c}\text { Parasitic } \\
\text { Animals }\end{array}$ & Crustacea & $\begin{array}{c}\text { Other } \\
\text { Animals }\end{array}$ \\
\hline $\begin{array}{l}15^{\circ} 44^{\prime} \mathrm{N} \\
172^{\circ} 20^{\prime} \mathrm{W}\end{array}$ & $\begin{array}{l}\text { Apriz } \\
19, \\
65\end{array}$ & $\begin{array}{l}\text { Pacific } \\
\text { Ocean }\end{array}$ & 1 & 1 & 1 & - & & & \\
\hline
\end{tabular}

Parasitic Animals:

lots: 4 squid

Notes on Squid:

1. Squid in fair condition; head attached.

2. Squid remains with tentacles.

squid beaks

squid remains

fish

Notes on Fish:

1. Head digested.

General Notes:

PACIFIC OCEANOGRAPHIC BIOLOGICAL SURVEY PROGRAM

SMITHSONIAN OCEANOGRAPHIC SORTING CENTER—SMITHSONIAN INSTITUTION

S1.USNM-630

P. Grisham 9/12/66

7.68

3.

\begin{tabular}{|c|c|c|c|c|c|c|c|c|c|}
\hline Sta. No. & 1001 & & Specie & Ster & fusc & & & $\begin{array}{l}\text { NO. } \\
\text { NO. }\end{array}$ & \\
\hline $\begin{array}{l}\text { Lat \& } \\
\text { Long. }\end{array}$ & Date & $\begin{array}{c}\text { Time } \\
\text { Caught }\end{array}$ & Squid & $\begin{array}{l}\text { Squid } \\
\text { Beaks }\end{array}$ & Fish & $\begin{array}{c}\text { Fish } \\
\text { Remains }\end{array}$ & $\begin{array}{l}\text { Parasitic } \\
\text { Animals }\end{array}$ & Crustacea & $\begin{array}{c}\text { Other } \\
\text { Animals }\end{array}$ \\
\hline $\begin{array}{l}15^{\circ} 44^{\prime} \mathrm{N} \\
272^{\circ} 20^{\prime} \mathrm{W}\end{array}$ & $\begin{array}{l}\text { April } \\
18, \\
65\end{array}$ & $\begin{array}{l}\text { Pacific } \\
\text { Ocean }\end{array}$ & 1 & 4 & & + & & & \\
\hline
\end{tabular}

Notes on Squid:

Fair condition; head attached.

Iats: 4 squid beaks eyeballs (2) Fish remains

Notes on Fish: squid

Fish remains with bones.

General Notes:

PACIFIC OCEANOGRAPHIC BIOLOGICAL SURVEY PROGRAM

SMITHSONIAN OCEANOGRAPHIC SORTING CENTER—SMITHSONIAN INSTITUTION

$51-45 \mathrm{NM}-830$

P. urisham $9 / 6 / 66$ 
SOSC REF. NO.

229

\begin{tabular}{|c|c|c|c|c|c|c|c|c|c|}
\hline Sta. No. & 1002 & & Species & Ste & fus & & & NO. & \\
\hline $\begin{array}{l}\text { Lat \& } \\
\text { Long. }\end{array}$ & Date & $\begin{array}{l}\text { Time } \\
\text { Caught }\end{array}$ & Squid & $\begin{array}{l}\text { Squid } \\
\text { Beaks }\end{array}$ & Fish & $\begin{array}{c}\text { Fish } \\
\text { Remains }\end{array}$ & $\begin{array}{l}\text { Parasitic } \\
\text { Animals }\end{array}$ & Crustacea & $\begin{array}{l}\text { Other } \\
\text { Animals }\end{array}$ \\
\hline $\begin{array}{l}15^{\circ} \mathrm{L}^{\prime} \mathrm{N} \\
172^{\circ} 20^{\prime} \mathrm{W}\end{array}$ & $\begin{array}{l}\text { April } \\
18, \\
65\end{array}$ & $\begin{array}{l}\text { Pacific } \\
\text { Ocean }\end{array}$ & & 3 & & + & & & \\
\hline
\end{tabular}

Parasitic Animals:

lots: 3 squid beaks

Notes on Squid:

fish remains

eyeballs (2)

Notes on Fish:

Fish remains with bones.

General Notes:

PACIFIC OCEANOGRAPHIC BIOLOGICAL SURVEY PROGRAM

SMITHSONIAN OCEANOGRAPHIC SORTING CENTER—SMITHSONIAN INSTITUTION

S1.USNM- 30

P. Grisham 9/12/66

$7 \cdot 65$

\begin{tabular}{|c|c|c|c|c|c|c|c|c|c|}
\hline Sta. No. & \multicolumn{2}{|c|}{1003} & Species & \multicolumn{3}{|c|}{ Sterna fuscata } & \multicolumn{2}{|c|}{ POBSP. NO. } & \multirow[b]{2}{*}{$\begin{array}{l}\text { Other } \\
\text { Animals }\end{array}$} \\
\hline $\begin{array}{l}\text { Lat \& } \\
\text { Long. }\end{array}$ & Date & $\begin{array}{l}\text { Time } \\
\text { Caught }\end{array}$ & Squid & $\begin{array}{l}\text { Squid } \\
\text { Beaks }\end{array}$ & Fish & $\begin{array}{c}\text { Fish } \\
\text { Remains }\end{array}$ & $\begin{array}{l}\text { Parasitic } \\
\text { Animals }\end{array}$ & Crustacea & \\
\hline $\begin{array}{l}15^{\circ} 44^{\prime} \mathrm{N} \\
172^{\circ} 20^{\prime} \mathrm{W}\end{array}$ & $\begin{array}{l}\text { April } \\
18, \\
65\end{array}$ & $\begin{array}{l}\text { Pacific } \\
\text { Ocean }\end{array}$ & 1 & 3 & & $t$ & & & \\
\hline
\end{tabular}

Parasitic Animals:

lots: 4 squid squid beaks

Notes on Squid: Fair condition, head slightly detached. digested remains eyebalis (1)

Notes on Fish:

General Notes:

Digested remains with a few fish bones.

PACIFIC OCEANOGRAPHIC BIOLOGICAL SURVEY PROGRAM SMITHSONIAN OCEANOGRAPHIC SORTING CENTER_SMITHSONIAN INSTITUT 


\begin{tabular}{|c|c|c|c|c|c|c|c|c|c|}
\hline $\begin{array}{l}\text { Lat \& } \\
\text { Long. }\end{array}$ & Date & $\begin{array}{l}\text { Time } \\
\text { Caught }\end{array}$ & Squid & $\begin{array}{l}\text { Squid } \\
\text { Beaks }\end{array}$ & Fish & $\begin{array}{c}\text { Fish } \\
\text { Remains }\end{array}$ & $\begin{array}{l}\text { Parasitic } \\
\text { Animals }\end{array}$ & Crustacea & $\begin{array}{c}\text { Other } \\
\text { Animals }\end{array}$ \\
\hline $\begin{array}{l}15^{\circ} 44^{\prime} \mathrm{N} \\
172^{\circ} 20^{\prime} \mathrm{W}\end{array}$ & $\begin{array}{l}\text { Apri1 } \\
18, \\
65\end{array}$ & $\begin{array}{l}\text { Pacific } \\
\text { Ocean }\end{array}$ & & & & $\frac{1}{+}$ & & & \\
\hline
\end{tabular}

Parasitic Animals:

lots: 2 fish remains

Notes on Squid:

Squid remains with tentacles.

squid remains

Notes on Fish:

Fish remains with bones.

General Notes:

PACIFIC OCEANOGRAPHIC BIOLOGICAL SURVEY PROGRAM SMITHSONIAN OCEANOGRAPHIC SORTING CENTER—SMITHSONIAN INSTITUTION

S1-USNM-830 P. Grisham 9/6/66

7-6s

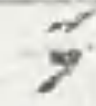

SOSC REF. NO. 229

\begin{tabular}{|c|c|c|c|c|c|c|c|c|c|}
\hline Sta. No. & 1005 & & Species & St & 12 fu & ta & & NO. & \\
\hline $\begin{array}{l}\text { Lat \& } \\
\text { Long. }\end{array}$ & Date & $\begin{array}{l}\text { Time } \\
\text { Caught }\end{array}$ & Squid & $\begin{array}{l}\text { Squid } \\
\text { Beaks }\end{array}$ & Fish & $\begin{array}{c}\text { Fish } \\
\text { Remains }\end{array}$ & $\begin{array}{l}\text { Parasitic } \\
\text { Animals }\end{array}$ & Crustacea & $\begin{array}{c}\text { Other } \\
\text { Animals }\end{array}$ \\
\hline $\begin{array}{l}15^{\circ} 4_{4}^{\prime} \mathrm{N} \\
172^{\circ} 20^{\prime} \mathrm{W}\end{array}$ & $\begin{array}{l}\text { April } \\
28, \\
65\end{array}$ & $\begin{array}{l}\text { Pacific } \\
\text { Ocean }\end{array}$ & & 7 & & + & & & \\
\hline
\end{tabular}

Parasitic Animals:

lots: 2 squid beaks

Notes on Squid:

fish remains

Notes on Fish:

Fish remains with bones.

General Notes:

PACIFIC OCEANOGRAPHIC BIOLOGICAL SURVEY PROGRAM

SMITHSONIAN OCEANOGRAPHIC SORTING CENTER-SMITHSONIAN INSTITUTION

S1-USNM-\$ 30

7-68 


\begin{tabular}{|c|c|c|c|c|c|c|c|c|c|}
\hline Sta. No. & 1006 & & Specie & Ster & fusc & & SOS & $\begin{array}{l}\text { NO. } \\
\text { NO. }\end{array}$ & \\
\hline $\begin{array}{l}\text { Lat \& } \\
\text { Long. }\end{array}$ & Date & $\begin{array}{l}\text { Time } \\
\text { Caught }\end{array}$ & Squid & $\begin{array}{l}\text { Squid } \\
\text { Beaks }\end{array}$ & Fish & $\begin{array}{c}\text { Fish } \\
\text { Remains }\end{array}$ & $\begin{array}{l}\text { Parasitic } \\
\text { Animals }\end{array}$ & Crustacea & $\begin{array}{l}\text { Other } \\
\text { Animals }\end{array}$ \\
\hline $\begin{array}{l}15^{\circ} 44^{\prime} \mathrm{N} \\
172^{\circ} 20^{\prime} \mathrm{W}\end{array}$ & $\begin{array}{l}\text { April } \\
18, \\
65\end{array}$ & $\begin{array}{l}\text { Pacific } \\
\text { Ocean }\end{array}$ & & & & - & & & \\
\hline
\end{tabular}

Parasitic Animals:

lots: 2 eyebalis (I)

Notes on Squid:

digested remains

\section{Notes on Fish:}

General Notes:

PACIFIC OCEANOGRAPHIC BIOLOGICAL SURVEY PROGRAM SMITHSONIAN OCEANOGRAPHIC SORTING CENTER_SMITHSONIAN INSTITUTION

SOSC REF. NO. 229

POBSP. NO. 10

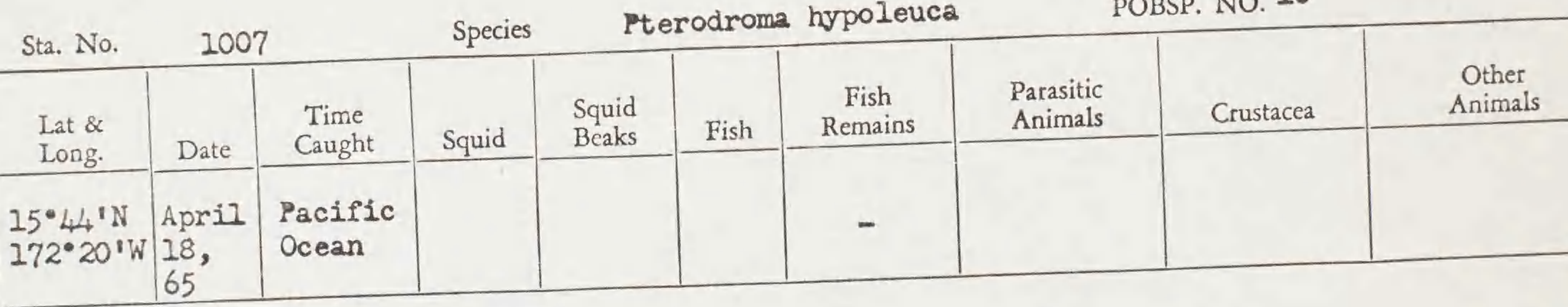

Parasitic Animals:

lots: 1 digested remains

Notes on Squid:

Notes on Fish:

General Notes:

Digested remains beyond identification.

PACIFIC OCEANOGRAPHIC BIOLOGICAL SURVEY PROGRAM

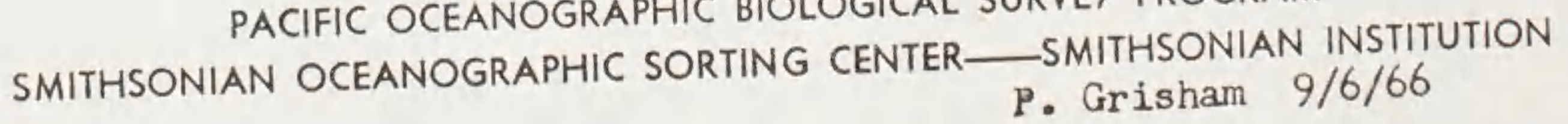


SOSC REF. NO. 229

\begin{tabular}{|c|c|c|c|c|c|c|c|c|c|}
\hline $\begin{array}{l}\text { Lat \& } \\
\text { Long. }\end{array}$ & Date & $\begin{array}{l}\text { Time } \\
\text { Caught }\end{array}$ & Squid & $\begin{array}{l}\text { Squid } \\
\text { Beaks }\end{array}$ & Fish & $\begin{array}{c}\text { Fish } \\
\text { Remains }\end{array}$ & $\begin{array}{l}\text { Parasitic } \\
\text { Animals }\end{array}$ & Crustacea & $\begin{array}{l}\text { Other } \\
\text { Animals }\end{array}$ \\
\hline $\begin{array}{l}15^{\circ} 38^{\prime} \mathrm{N} \\
173^{\circ} 39^{\prime} \mathrm{W}\end{array}$ & $\begin{array}{l}\text { April } \\
19, \\
65\end{array}$ & $\begin{array}{l}\text { Pacific } \\
\text { Ocean }\end{array}$ & & & & - & & & \\
\hline
\end{tabular}

Parasitic Animals:

lots: 0

Notes on Squid:

Notes on Fish:

General Notes:

The stomach was empty.

PACIFIC OCEANOGRAPHIC BIOLOGICAL SURVEY PROGRAM

SMITHSONIAN OCEANOGRAPHIC SORTING CENTER—SMITHSONIAN INSTITUTION

P. Grisham 9/6/66

7-60

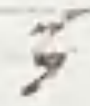

\begin{tabular}{|c|c|}
\hline $\begin{array}{l}\text { Lat \& } \\
\text { Long. }\end{array}$ & Date \\
\hline $\begin{array}{l}17^{\circ} 16^{\prime} \mathrm{N} \\
171^{\circ} 10^{\prime} \mathrm{W}\end{array}$ & $\begin{array}{l}\text { April } \\
20, \\
65\end{array}$ \\
\hline
\end{tabular}

1010

Species

Sterna fuscata

, SOSC REF. NO. 229

\begin{tabular}{|c|}
\hline $\begin{array}{l}\text { Time } \\
\text { Caught }\end{array}$ \\
\hline $\begin{array}{l}\text { Pacific } \\
\text { Ocean }\end{array}$ \\
\hline
\end{tabular}

Squid

POBSP. NO.

10

Notes on Squid:

lots: 0

Notes on Fish:

General Notes:

The stomach was empty.

PACIFIC OCEANOGRAPHIC BIOLOGICAL SURVEY PROGRAM

SMITHSONIAN OCEANOGRAPHIC SORTING CENTER—SMITHSONIAN INSTJTUTION

Si.USNM-830 


\begin{tabular}{|c|c|c|c|c|c|c|c|c|c|}
\hline Sta. No. & 101 & & Speci & & nus & carneipes & & $\begin{array}{l}\text { NO. } \\
\text { NO. }\end{array}$ & \\
\hline $\begin{array}{l}\text { Lat \& } \\
\text { Long. }\end{array}$ & Date & $\begin{array}{l}\text { Time } \\
\text { Caught }\end{array}$ & Squid & $\begin{array}{l}\text { Squid } \\
\text { Beaks }\end{array}$ & Fish & $\begin{array}{c}\text { Fish } \\
\text { Remains }\end{array}$ & $\begin{array}{l}\text { Parasitic } \\
\text { Animals }\end{array}$ & Crustacea & $\begin{array}{c}\text { Other } \\
\text { Animals }\end{array}$ \\
\hline $\begin{array}{l}17^{\circ} 28^{\prime} \mathrm{N} \\
170^{\circ} 31^{\prime} \mathrm{W}\end{array}$ & $\begin{array}{l}\text { April } \\
20, \\
65\end{array}$ & $\begin{array}{l}\text { Pacific } \\
\text { Ocean }\end{array}$ & 1 & 30 & & - & & & \\
\hline
\end{tabular}

Parasitic Animals:

Lots: 3 squid beaks

Notes on Squid:

digested remains

eyeballs (4)

Notes on Fish:

General Notes:

PACIFIC OCEANOGRAPHIC BIOLOGICAL SURVEY PROGRAM SMITHSONIAN OCEANOGRAPHIC SORTING CENTER — SMITHSONIAN INSTITUTION

S1. USNAM-630

P. Grisham 9/12/66

Si. 0.65
7.65

$-2$

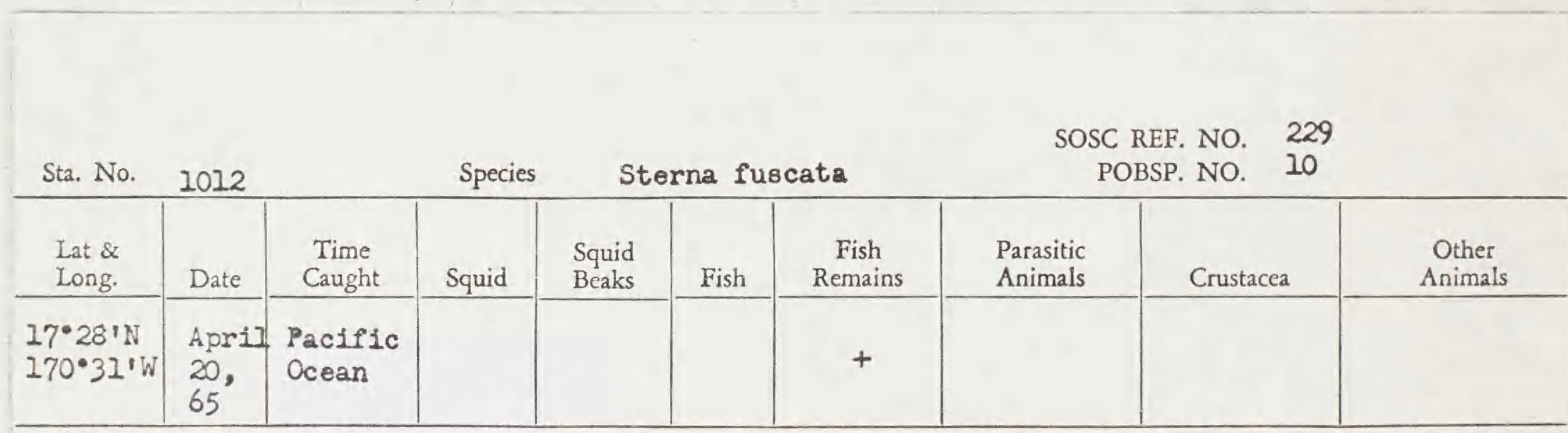

Parasitic Animals:

Notes on Squid:

lots: 1 fish remains

Notes on Fish:

Fish remains with bones.

General Notes:

PACIFIC OCEANOGRAPHIC BIOLOGICAL SURVEY PROGRAM

SMITHSONIAN OCEANOGRAPHIC SORTING CENTER — SMITHSONIAN INSTITUTION

51. USNM-830

P. Grisham 8/31/66 


\begin{tabular}{|c|c|c|c|c|c|c|c|c|c|}
\hline Sta. No. & 1013 & & Speci & & cna 1 & cata & $\begin{array}{r}\text { SOSC } \\
\text { PC }\end{array}$ & $\begin{array}{l}\text { NO. } \\
\text { NO. }\end{array}$ & \\
\hline $\begin{array}{l}\text { Lat \& } \\
\text { Long. }\end{array}$ & Date & $\begin{array}{l}\text { Time } \\
\text { Caught }\end{array}$ & Squid & $\begin{array}{l}\text { Squid } \\
\text { Beaks }\end{array}$ & Fish & $\begin{array}{c}\text { Fish } \\
\text { Remains }\end{array}$ & $\begin{array}{l}\text { Parasitic } \\
\text { Animals }\end{array}$ & Crustacea & $\begin{array}{c}\text { Other } \\
\text { Animals }\end{array}$ \\
\hline $\begin{array}{l}17^{\circ} 28^{\prime} \mathrm{N} \\
170^{\circ} 32^{\prime} \mathrm{W}\end{array}$ & $\begin{array}{l}\text { April } \\
20 \\
65\end{array}$ & $\begin{array}{l}\text { Pacific } \\
\text { Ocean }\end{array}$ & & 1 & 1 & + & $\begin{array}{c}\text { Nematodes } \\
\text { (1) }\end{array}$ & & \\
\hline
\end{tabular}

Parasitic Animals:

Notes on Squid:

lots: 4 scuid beaks

\section{fish}

fish remains

nematode

Notes on Fish:

1. Fish remains with bones.

2. Head digested.

General Notes:

PACIFIC OCEANOGRAPHIC BIOLOGICAL SURVEY PROGRAM

SMITHSONIAN OCEANOGRAPHIC SORTING CENTER_-SMITHSONIAN INSTITUTION

S1. USNM-830

7.65

\begin{tabular}{|c|c|c|c|c|c|c|c|c|c|}
\hline \multirow{2}{*}{$\begin{array}{l}\text { Sta. No. } \\
\text { Lat \& } \\
\text { Long. }\end{array}$} & \multicolumn{2}{|c|}{1014} & Species & \multicolumn{3}{|c|}{ Sterna fuscata } & \multicolumn{2}{|c|}{$\begin{aligned} & \text { SOSC REF. NO. } 229 \\
& \text { POBSP. NO. } 10 \\
&\end{aligned}$} & \multirow[b]{2}{*}{$\begin{array}{l}\text { Other } \\
\text { Animals }\end{array}$} \\
\hline & Date & $\begin{array}{l}\text { Time } \\
\text { Caught }\end{array}$ & Squid & $\begin{array}{l}\text { Squid } \\
\text { Beaks }\end{array}$ & Fish & $\begin{array}{c}\text { Fish } \\
\text { Remains }\end{array}$ & $\begin{array}{l}\text { Parasitic } \\
\text { Animals }\end{array}$ & Crustacea & \\
\hline $\begin{array}{l}17^{\circ} 28^{\circ} \mathrm{N} \\
170^{\circ} 32^{\prime} \mathrm{W}\end{array}$ & $\begin{array}{l}\text { April } \\
20, \\
65\end{array}$ & $\begin{array}{l}\text { Pacific } \\
\text { Ocean }\end{array}$ & & & & + & & & \\
\hline
\end{tabular}

Parasitic Animals:

lots: 1 fish remains

Notes on Squid:

Notes on Fish:

Fish remains with bones.

General Notes:

\footnotetext{
PACIFIC OCEANOGRAPHIC BIOLOGICAL SURVEY PROGRAM

SMITHSONIAN OCEANOGRAPHIC SORTING CENTER-SMITHSONIAN INSTITUTION
} 


\begin{tabular}{|c|c|c|c|c|c|c|c|c|c|}
\hline $\begin{array}{l}\text { Lat \& } \\
\text { Long. }\end{array}$ & Date & $\begin{array}{l}\text { Time } \\
\text { Caught }\end{array}$ & Squid & $\begin{array}{l}\text { Squid } \\
\text { Beaks }\end{array}$ & Fish & $\begin{array}{c}\text { Fish } \\
\text { Remains }\end{array}$ & $\begin{array}{l}\text { Parasitic } \\
\text { Animals }\end{array}$ & Crustacea & $\begin{array}{l}\text { Other } \\
\text { Animals }\end{array}$ \\
\hline $\begin{array}{l}17^{\circ} 28^{\prime} \mathrm{N} \\
170^{\circ} 31^{\prime} \mathrm{W}\end{array}$ & $\begin{array}{l}\text { April } \\
20, \\
65\end{array}$ & $\begin{array}{l}\text { Pacific } \\
\text { ocean }\end{array}$ & 3 & & & + & & & \\
\hline
\end{tabular}

Parasitic Animals:

lots: 3 squids
Notes on Squid:
1. Heads of two digested.
2. Squid remains with tentacles.

\section{Notes on Fish:}

General Notes:

PACIFIC OCEANOGRAPHIC BIOLOGICAL SURVEY PROGRAM SMITHSONIAN OCEANOGRAPHIC SORTING CENTER — SMITHSONIAN INSTITUTION

$$
\text { P. Grisham } 8 / 16 / 66
$$

SOSC REF. NO. 229

\begin{tabular}{|c|c|c|c|c|c|c|c|c|c|}
\hline \multirow{2}{*}{$\begin{array}{l}\text { Sta. No. } \\
\text { Lat \& } \\
\text { Long. }\end{array}$} & \multicolumn{2}{|l|}{1016} & Species & \multicolumn{3}{|c|}{ Sterna fuscata } & \multicolumn{2}{|c|}{ POBSP. NO. IO } & \multirow[b]{2}{*}{$\begin{array}{c}\text { Other } \\
\text { Animals }\end{array}$} \\
\hline & Date & $\begin{array}{l}\text { Time } \\
\text { Caught }\end{array}$ & Squid & $\begin{array}{l}\text { Squid } \\
\text { Beaks }\end{array}$ & Fish & $\begin{array}{c}\text { Fish } \\
\text { Remains }\end{array}$ & $\begin{array}{l}\text { Parasitic } \\
\text { Animals }\end{array}$ & Crustacea & \\
\hline $\begin{array}{l}17^{\circ} 28^{\prime} \mathrm{N} \\
170^{\circ} 31^{\prime} \mathrm{W}\end{array}$ & $\begin{array}{l}\text { April } \\
20, \\
65\end{array}$ & $\begin{array}{l}\text { Pacific } \\
\text { Ocean }\end{array}$ & 2 & 3 & & + & & & \\
\hline
\end{tabular}

Parasitic Animals:

Notes on Squid:

Head of one detached.

lots: 3 fish remains

squids

squid beaks

Notes on Fish: Fish remains with pieces of fish.

General Notes:

PACIFIC OCEANOGRAPHIC BIOLOGICAL SURVEY PROGRAM SMITHSONIAN OCEANOGRAPHIC SORTING CENTER—SMITHSONIAN INSTJTUTIQN 
SOSC REF. NO.

229

\begin{tabular}{|c|c|c|c|c|c|c|c|c|c|}
\hline $\begin{array}{l}\text { Lat \& } \\
\text { Long. }\end{array}$ & Date & $\begin{array}{l}\text { Time } \\
\text { Caught }\end{array}$ & Squid & $\begin{array}{l}\text { Squid } \\
\text { Beaks }\end{array}$ & Fish & $\begin{array}{c}\text { Fish } \\
\text { Remains }\end{array}$ & $\begin{array}{l}\text { Parasitic } \\
\text { Animals }\end{array}$ & Crustacea & $\begin{array}{l}\text { Other } \\
\text { Animals }\end{array}$ \\
\hline $\begin{array}{l}17^{\circ} 28^{\prime} \mathrm{N} \\
170^{\circ} 31^{\prime} \mathrm{W}\end{array}$ & $\begin{array}{l}\text { April } \\
20 \\
65\end{array}$ & $\begin{array}{l}\text { Pačific } \\
\text { Ocean }\end{array}$ & 1 & 1 & & + & & & \\
\hline
\end{tabular}

Parasitic Animals:

lots: 3 squid beaks

Notes on Squid:

1. Head attached; fair condition.

fish remains squid

Notes on Fish:

I. Fish remains with bones.

General Notes:

PACIFIC OCEANOGRAPHIC BIOLOGICAL SURVEY PROGRAM

SMITHSONIAN OCEANOGRAPHIC SORTING CENTER_SMITHSONIAN INSTITUTION

S1.USNM-830

$7 \rightarrow 05$

P. Grisham 9/12/66

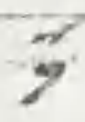

Sta. No.

1018

Sterna fuscata

SOSC REF. NO. 229

\begin{tabular}{|c|c|c|c|c|c|c|c|c|c|}
\hline $\begin{array}{l}\text { Lat \& } \\
\text { Long. }\end{array}$ & Date & $\begin{array}{l}\text { Time } \\
\text { Caught }\end{array}$ & Squid & $\begin{array}{l}\text { Squid } \\
\text { Beaks }\end{array}$ & Fish & $\begin{array}{c}\text { Fish } \\
\text { Remains }\end{array}$ & $\begin{array}{l}\text { Parasitic } \\
\text { Animals }\end{array}$ & Crustacea & $\begin{array}{l}\text { Other } \\
\text { Animals }\end{array}$ \\
\hline $\begin{array}{l}17^{\circ} 28^{\prime} \mathrm{N} \\
170^{\circ} 31^{\prime} \mathrm{W}\end{array}$ & $\begin{array}{l}\text { April } \\
20, \\
65\end{array}$ & $\begin{array}{l}\text { Pacific } \\
\text { Ocean }\end{array}$ & & & & + & & & \\
\hline
\end{tabular}

Parasitic Animals:

lots: 1 fish remains

Notes on Squid:

Notes on Fish:

Fish remains with bones.

General Notes:

PACIFIC OCEANOGRAPHIC BIOLOGICAL SURVEY PROGRAM

SMITHSONIAN OCEANOGRAPHIC SORTING CENTER—SMITHSONIAN INSTITUTION

S1-USNM-630

P. Grisham $9 / 12 / 66$ 


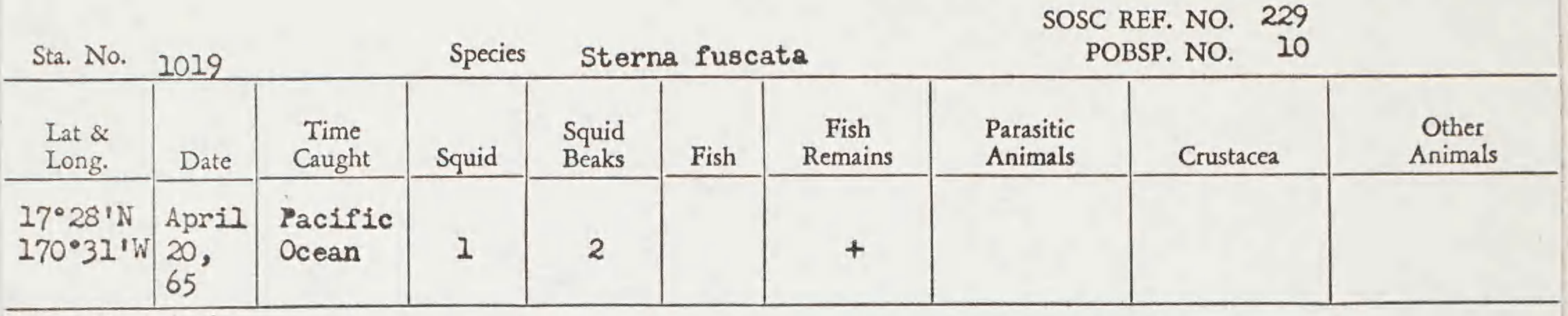

Parasitic Animals:

lots: 3 squid

Notes on Squid:

Fair; head attached. squid beaks fish remains

Notes on Fish:

General Notes:

PACIFIC OCEANOGRAPHIC BIOLOGICAL SURVEY PROGRAM

SMITHSONIAN OCEANOGRAPHIC SORTING CENTER — SMITHSONIAN INSTITUTION

SI-USNM-830

P.Grisham $9 / 13 / 66$

$\operatorname{tin}_{-65}$

SOSC REF. NO. 229

POBSP. NO. 10

\begin{tabular}{c|c|c|c|c|c|c} 
Sta. No. & \multicolumn{2}{c}{1020} & \multicolumn{2}{c}{ Species } & \multicolumn{2}{c}{ Sterna fuscata } \\
$\begin{array}{c}\text { Lat \& } \\
\text { Long. }\end{array}$ & Date & $\begin{array}{l}\text { Time } \\
\text { Caught }\end{array}$ & Squid & $\begin{array}{l}\text { Squid } \\
\text { Beaks }\end{array}$ & Fish & $\begin{array}{c}\text { Fish } \\
\text { Remains }\end{array}$ \\
\hline $\begin{array}{l}17^{\circ} 28^{\prime} \mathrm{N} \\
170^{\circ} 32^{\prime} \mathrm{W}\end{array}$ & $\begin{array}{l}\text { April } \\
20, \\
65\end{array}$ & $\begin{array}{l}\text { Pacific } \\
\text { Ocean }\end{array}$ & 1 & & & + \\
\end{tabular}

Parasitic Animals:

lots: 2 squid

Notes on Squid:

Head digested.

fish remains

Notes on Fish:

General Notes:

PACIFIC OCEANOGRAPHIC BIOLOGICAL SURVEY PROGRAM SMITHSONIAN OCEANOGRAPHIC SORTING CENTER—SMITHSONIAN INSTITUTION 
SOSC REF. NO.

\begin{tabular}{|c|c|c|c|c|c|c|c|c|c|}
\hline Sta. No. & $102]$ & & Specie & Stern & fusc: & & & NO. & \\
\hline $\begin{array}{l}\text { Lat \& } \\
\text { Long. }\end{array}$ & Date & $\begin{array}{l}\text { Time } \\
\text { Caught }\end{array}$ & Squid & $\begin{array}{l}\text { Squid } \\
\text { Beaks }\end{array}$ & Fish & $\begin{array}{c}\text { Fish } \\
\text { Remains }\end{array}$ & $\begin{array}{l}\text { Parasitic } \\
\text { Animals }\end{array}$ & Crustacea & $\begin{array}{l}\text { Other } \\
\text { Animals }\end{array}$ \\
\hline $\begin{array}{r}17^{\circ} 28^{\prime} \mathrm{N} \\
170^{\circ} 3 I^{\prime} \mathrm{W}\end{array}$ & $\begin{array}{l}\text { Apri] } \\
20, \\
65\end{array}$ & $\begin{array}{l}\text { Pacific } \\
\text { Ocean }\end{array}$ & 1 & & & - & & & \\
\hline
\end{tabular}

Parasitic Animals:

lots: 2 squid

Notes on Squid:

Head detachød.

digested remains

\section{Notes on Fish:}

General Notes:

PACIFIC OCEANOGRAPHIC BIOLOGICAL SURVEY PROGRAM

SMITHSONIAN OCEANOGRAPHIC SORTING CENTER—SMITHSONIAN INSTITUTION

S1.USNM-\$30

P. Grisham 9/6/66

Ti-0s

5

SOSC REF. NO. 229

POBSP. NO. 10

\begin{tabular}{|c|c|c|c|c|c|c|c|c|c|}
\hline Sta. No. & \multicolumn{3}{|r|}{ Species } & \multicolumn{3}{|c|}{ Sterna fuscata } & \multicolumn{2}{|c|}{ POBSP. NO. 10} & \multirow[b]{2}{*}{$\begin{array}{c}\text { Other } \\
\text { Animals }\end{array}$} \\
\hline $\begin{array}{l}\text { Lat \& } \\
\text { Long. }\end{array}$ & Date & $\begin{array}{l}\text { Time } \\
\text { Caught }\end{array}$ & Squid & $\begin{array}{l}\text { Squid } \\
\text { Beaks }\end{array}$ & Fish & $\begin{array}{c}\text { Fish } \\
\text { Remains }\end{array}$ & $\begin{array}{l}\text { Parasitic } \\
\text { Animals }\end{array}$ & Crustacea & \\
\hline $\begin{array}{l}17^{\circ} 28^{\prime} \mathrm{N} \\
170^{\circ} 31^{\prime} \mathrm{W}\end{array}$ & $\begin{array}{l}\text { April } \\
20, \\
65\end{array}$ & $\begin{array}{l}\text { Pacific } \\
\text { Ocean }\end{array}$ & 2 & & & + & & & \\
\hline
\end{tabular}

Parasitic Animals:

1ots: 3 squids

squid remains

Notes on Squid:

Heads detached.

fish remains

Notes on Fish:

General Notes:

PACIFIC OCEANOGRAPHIC BIOLOGICAL SURVEY PROGRAM SMITHSONIAN OCEANOGRAPHIC SORTING CENTER—SMITHSONIAN INSTITUTION

P. Grisham 8/31/66 


\begin{tabular}{|c|c|c|c|c|c|c|c|c|c|}
\hline $\begin{array}{l}\text { Lat \& } \\
\text { Long. }\end{array}$ & Date & $\begin{array}{l}\text { Time } \\
\text { Caught }\end{array}$ & Squid & $\begin{array}{l}\text { Squid } \\
\text { Beaks }\end{array}$ & Fish & $\begin{array}{c}\text { Fish } \\
\text { Remains }\end{array}$ & $\begin{array}{l}\text { Parasitic } \\
\text { Animals }\end{array}$ & Crustacea & $\begin{array}{c}\text { Other } \\
\text { Animals }\end{array}$ \\
\hline $\begin{array}{l}17^{\circ} 28^{\prime} \mathrm{N} \\
170^{\circ} 32^{\prime} \mathrm{W}\end{array}$ & $\begin{array}{l}\text { April } \\
20, \\
65\end{array}$ & $\begin{array}{l}\text { Pacific } \\
\text { Ocean }\end{array}$ & $I$ & & & - & & & \\
\hline
\end{tabular}

Parasitic Animals:

lots: 2 squid

Notes on Squid:

Good condition, head attached.

Notes on Fish:

General Notes:

PACIFIC OCEANOGRAPHIC BIOLOGICAL SURVEY PROGRAM

SMITHSONIAN OCEANOGRAPHIC SORTING CENTER_SMITHSONIAN INSTITUTION

P. Grisham 8/29/66

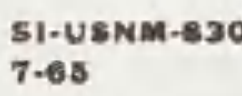

3

Sta. No.

1024

Species

Sterna fuscata

SOSC REF. NO. 229

\begin{tabular}{|c|c|c|c|c|c|c|c|c|c|}
\hline $\begin{array}{l}\text { Lat \& } \\
\text { Long. }\end{array}$ & Date & $\begin{array}{l}\text { Time } \\
\text { Caught }\end{array}$ & Squid & $\begin{array}{l}\text { Squid } \\
\text { Beaks }\end{array}$ & Fish & $\begin{array}{c}\text { Fish } \\
\text { Remains }\end{array}$ & $\begin{array}{l}\text { Parasitic } \\
\text { Animals }\end{array}$ & Crustacea & $\begin{array}{c}\text { Other } \\
\text { Animals }\end{array}$ \\
\hline $\begin{array}{l}17^{\circ} 28^{\prime} \mathrm{N} \\
170^{\circ} 31^{\prime} \mathrm{W}\end{array}$ & $\begin{array}{l}\text { April } \\
20,\end{array}$ & $\begin{array}{l}\text { Pacific } \\
\text { Ocean }\end{array}$ & & & & - & & & \\
\hline
\end{tabular}

Parasitic Animals:

lots: 1 digested remains

Notes on Squid:

Notes on Fish:

General Notes:

Digested remains beyond identification.

PACIFIC OCEANOGRAPHIC BIOLOGICAL SURVEY PROGRAM

SMITHSONIAN OCEANOGRAPHIC SORTING CENTER—SMITHSONIAN INSTITUTION

SI-USNM- -30
$7-65$

P. Grisham $8 / 4 / 66$ 
SOSC REF. NO. 229

Sta. No. 1025 Species POBSP. NO.

10

\begin{tabular}{|c|c|c|c|c|c|c|c|c|c|}
\hline $\begin{array}{l}\text { Lat \& } \\
\text { Long. }\end{array}$ & Date & $\begin{array}{c}\text { Time } \\
\text { Caught }\end{array}$ & Squid & $\begin{array}{l}\text { Squid } \\
\text { Beaks }\end{array}$ & Fish & $\begin{array}{c}\text { Fish } \\
\text { Remains }\end{array}$ & $\begin{array}{l}\text { Parasitic } \\
\text { Animals }\end{array}$ & Crustacea & $\begin{array}{c}\text { Other } \\
\text { Animals }\end{array}$ \\
\hline $\begin{array}{l}17^{\circ} 28^{\prime} \mathrm{N} \\
170^{\circ} 3 I^{\prime} \mathrm{W}\end{array}$ & $\begin{array}{l}\text { April } \\
20 \\
65\end{array}$ & $\begin{array}{l}\text { Pacific } \\
\text { Ocean }\end{array}$ & & & 1 & + & & & \\
\hline
\end{tabular}

Parasitic Animals:

lots: 2 fish (eel)

Notes on Squid:

fish remains
Notes on Fish:
1. Fish remains with bones.
2. Head of fish digested.

General Notes:

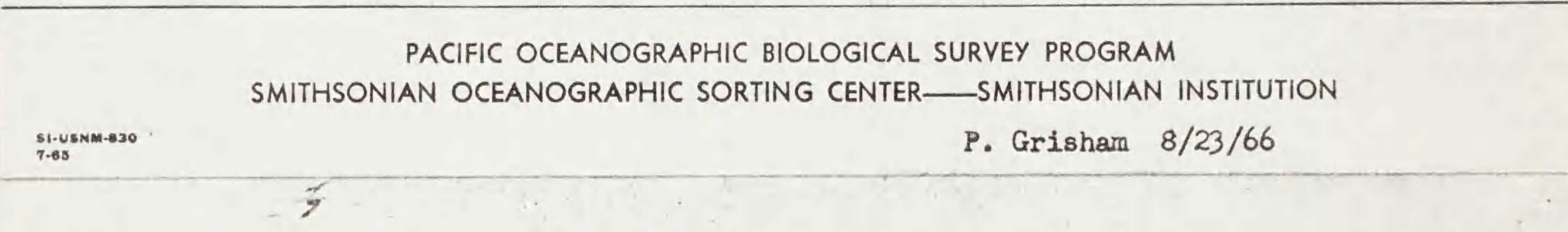

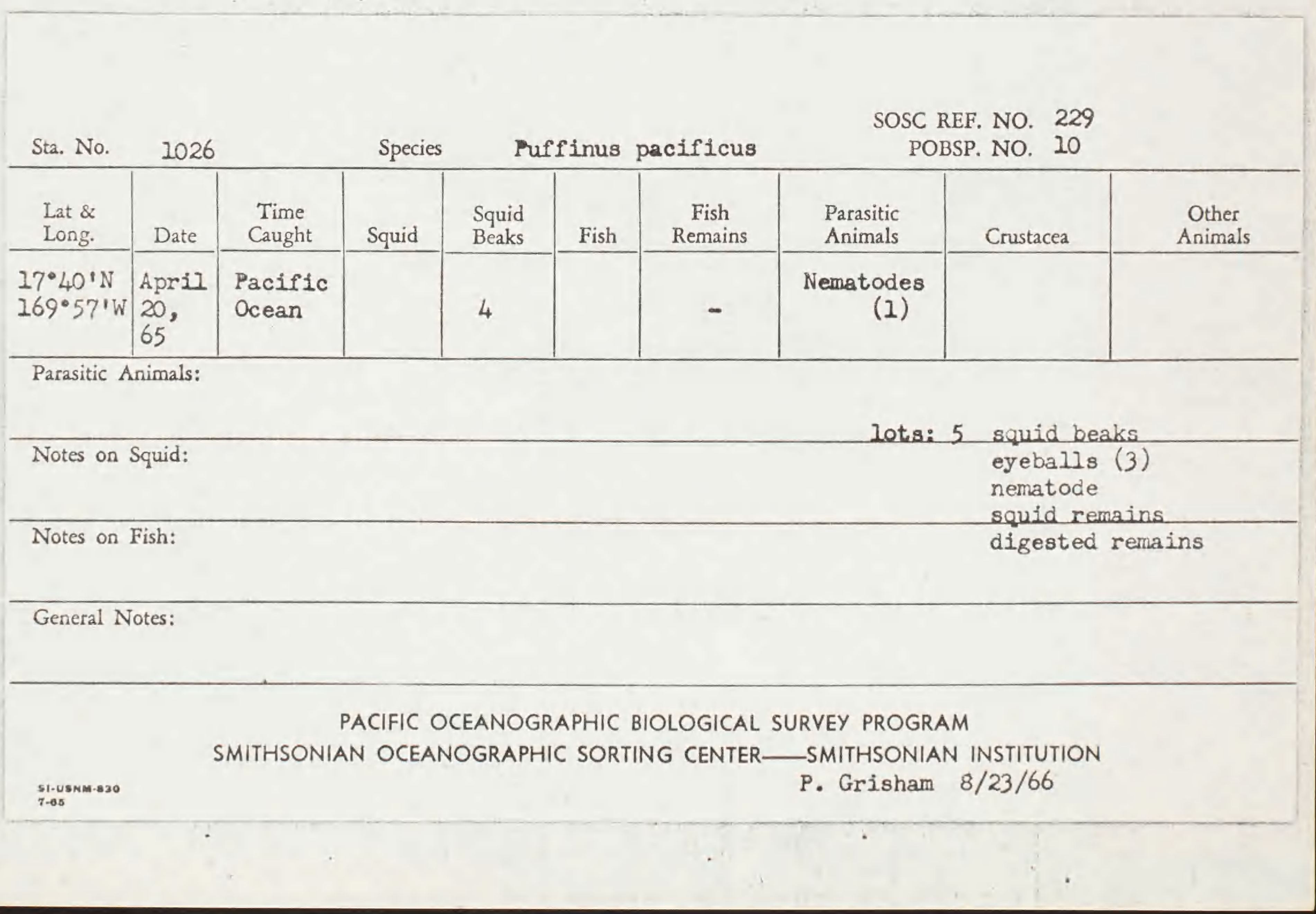




\begin{tabular}{|c|c|c|c|c|c|c|c|c|c|}
\hline Sta. No. & 1027 & & Speci & & inus & cificus & SO & $\begin{array}{l}\text { NO. } \\
\text { NO. }\end{array}$ & \\
\hline $\begin{array}{l}\text { Lat \& } \\
\text { Long. }\end{array}$ & Date & $\begin{array}{l}\text { Time } \\
\text { Caught }\end{array}$ & Squid & $\begin{array}{l}\text { Squid } \\
\text { Beaks }\end{array}$ & Fish & $\begin{array}{c}\text { Fish } \\
\text { Remains }\end{array}$ & $\begin{array}{l}\text { Parasitic } \\
\text { Animals }\end{array}$ & Crustacea & $\begin{array}{l}\text { Other } \\
\text { Animals }\end{array}$ \\
\hline $\begin{array}{l}17^{\circ} 40^{\prime} \mathrm{N} \\
169^{\circ} 57^{\prime} \mathrm{W}\end{array}$ & $\begin{array}{l}\text { April } \\
20, \\
65\end{array}$ & $\begin{array}{l}\text { Pacific } \\
\text { Ocean }\end{array}$ & & 7 & & - & & & \\
\hline
\end{tabular}

Parasitic Animals:

lots: 3 squid beaks

Notes on Squid: eyeballs ( 1 ) digested remains

Notes on Fish:

General Notes:

PACIFIC OCEANOGRAPHIC BIOLOGICAL SURVEY PROGRAM SMITHSONIAN OCEANOGRAPHIC SORTING CENTER—SMITHSONIAN INSTITUTION

P. Grisham 9/12/66

SOSC REF. NO. 229

POBSP. NO. 10

Sta. No.

1028

Species Sterna fuscata

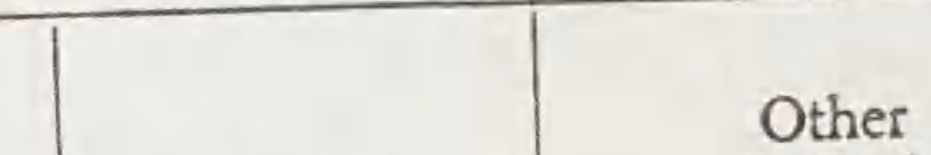

Lat \&

Long.

$17^{\circ} 42^{\circ} \mathrm{N}$ April

$169^{\circ} 47^{\prime} \mathrm{W} 20$,

65

\begin{tabular}{|c|c|} 
Fish & $\begin{array}{c}\text { Fish } \\
\text { Remains }\end{array}$ \\
+
\end{tabular}

\begin{tabular}{|c|c|c|}
$\begin{array}{c}\text { Parasitic } \\
\text { Animals }\end{array}$ & Crustacea & $\begin{array}{c}\text { Other } \\
\text { Animals }\end{array}$ \\
\hline & & \\
& & \\
& & \\
\hline
\end{tabular}

Parasitic Animals:

lots: I fish remains

Notes on Squid:

Notes on Fish:

Fish remains with bones.

General Notes:

PACIFIC OCEANOGRAPHIC BIOLOGICAL SURVEY PROGRAM

PACIFIC OCEANOGRAPHIC BIOLOGICAL SMITHSONIAN OCEANOGRAPHIC SORTING CENTER-SMITHSONIAN INSTITUTION
P. Grisham $8 / 16 / 66$ 


\begin{tabular}{|c|c|c|c|c|c|c|c|c|c|}
\hline Sta. No. & 1029 & & Spec & & $I_{\mathrm{a}} \mathrm{d}$ & ylatra & & $\begin{array}{l}\text { NO. } \\
\text { NO. }\end{array}$ & \\
\hline $\begin{array}{l}\text { Lat \& } \\
\text { Long. }\end{array}$ & Date & $\begin{array}{l}\text { Time } \\
\text { Caught }\end{array}$ & Squid & $\begin{array}{l}\text { Squid } \\
\text { Beaks }\end{array}$ & Fish & $\begin{array}{c}\text { Fish } \\
\text { Remains }\end{array}$ & $\begin{array}{l}\text { Parasitic } \\
\text { Animals }\end{array}$ & Crustacea & $\begin{array}{c}\text { Other } \\
\text { Animals }\end{array}$ \\
\hline $\begin{array}{l}18^{\circ} 38^{\prime} \mathrm{N} \\
166^{\circ} 51^{\prime} \mathrm{W}\end{array}$ & $\begin{array}{l}\text { April } \\
21, \\
65\end{array}$ & $\begin{array}{l}\text { Pacific } \\
\text { Ocean }\end{array}$ & & & 3 & + & & & \\
\hline
\end{tabular}

Parasitic Animals:

Notes on Squid:

lots: 4 fish

fish remains

eyeballs (1)

Notes on Fish:

1. Fish remains with half of a fish:

2. One fish in good condition and others are in fair condition.

General Notes:

PACIFIC OCEANOGRAPHIC BIOLOGICAL SURVEY PROGRAM

SMITHSONIAN OCEANOGRAPHIC SORTING CENTER_SMITHSONIAN INSTITUTION

P. Grisham 8/29/66

S1. U5NM-830

7.es

$\dddot{y}$

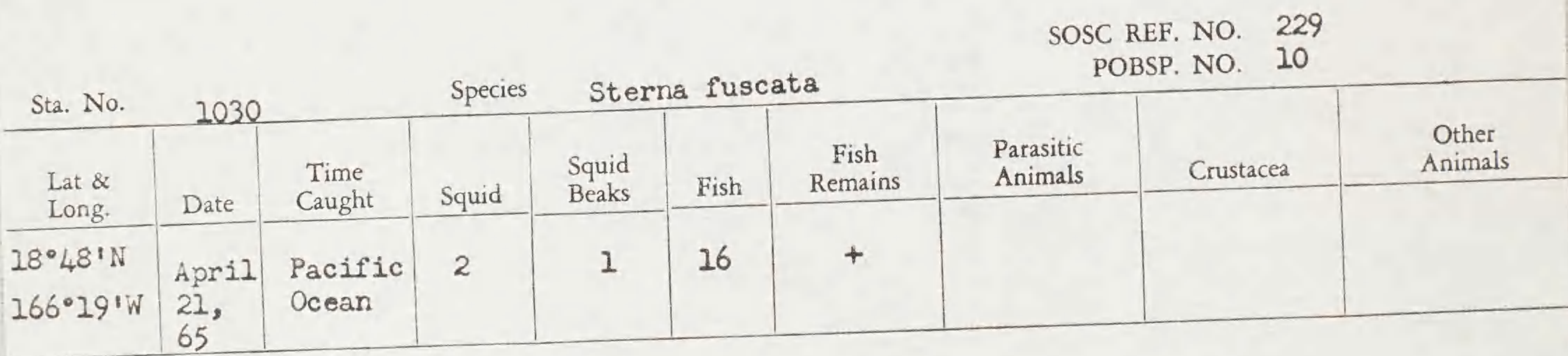

Parasitic Animals:

Notes on Squid: Head of one detached.

1ots: 4 squids

fish

scuid beaks

fish remains

Notes on Fish: Heads digested; poor condition.

General Notes:

PACIFIC OCEANOGRAPHIC BIOLOGICAL SURVEY PROGRAM SMITHSONIAN OCEANOGRAPHIC SORTING CENTER-SMITHSONIAN INSTJTUTION 
SOSC REF. NO. 229

\begin{tabular}{|c|c|c|c|c|c|c|c|c|c|}
\hline $\begin{array}{l}\text { Lat \& } \\
\text { Long. }\end{array}$ & Date & $\begin{array}{l}\text { Time } \\
\text { Caught }\end{array}$ & Squid & $\begin{array}{l}\text { Squid } \\
\text { Beaks }\end{array}$ & Fish & $\begin{array}{c}\text { Fish } \\
\text { Remains }\end{array}$ & $\begin{array}{l}\text { Parasitic } \\
\text { Animals }\end{array}$ & Crustacea & $\begin{array}{l}\text { Other } \\
\text { Animals }\end{array}$ \\
\hline $\begin{array}{l}18^{\circ} 48^{\prime} \mathrm{N} \\
166^{\circ} 19^{\prime} \mathrm{W}\end{array}$ & $\begin{array}{l}\text { Aprid } \\
21, \\
65\end{array}$ & $\begin{array}{l}\text { Pacific } \\
\text { Ocean }\end{array}$ & & & & + & & & \\
\hline
\end{tabular}

Parasitic Animals:

lots: 1 fish remains

Notes on Squid:

Notes on Fish:

Fish remains with bones.

General Notes:

PACIFIC OCEANOGRAPHIC BIOLOGICAL SURVEY PROGRAM

SMITHSONIAN OCEANOGRAPHIC SORTING CENTER_-SMITHSONIAN INSTITUTION

51-USNM-830

P. Grisham 9/6/66

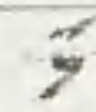

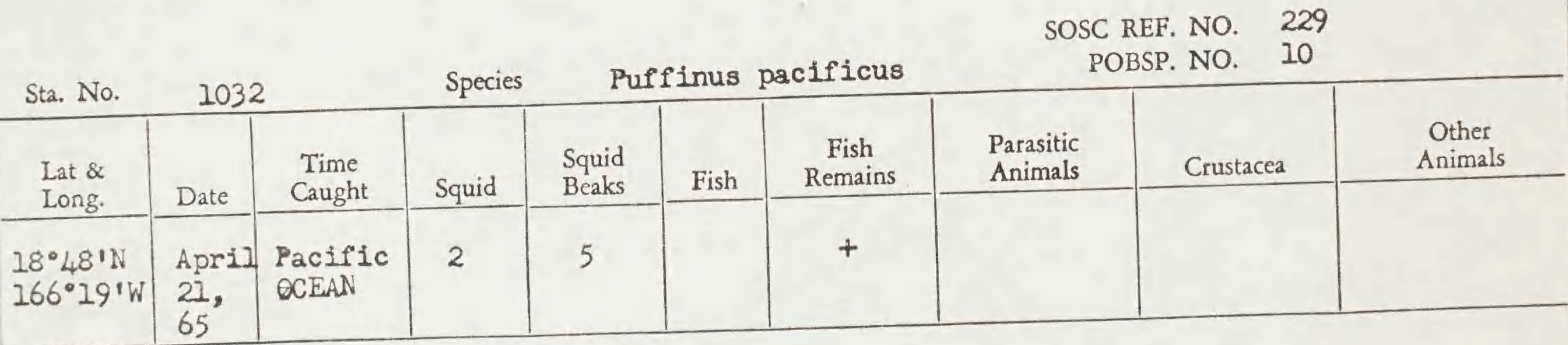

Parasitic Animals:

lots: 4 squid beaks squid remains

Notes on Squid: 1. Head of one digested. fish remains

2. Second in good condition. squids

Notes on Fish:

General Notes:

PACIFIC OCEANOGRAPHIC BIOLOGICAL SURVEY PROGRAM SMITHSONIAN OCEANOGRAPHIC SORTING CENTER—SMITHSONIAN INSTITUTION 
SOSC REF. NO. 229

Sta. No. Species

Sterna fuscata

POBSP. NO. 10

\begin{tabular}{|c|c|c|c|c|c|c|c|c|c|}
\hline $\begin{array}{l}\text { Lat \& } \\
\text { Long. }\end{array}$ & Date & $\begin{array}{l}\text { Time } \\
\text { Caught }\end{array}$ & Squid & $\begin{array}{l}\text { Squid } \\
\text { Beaks }\end{array}$ & Fish & $\begin{array}{c}\text { Fish } \\
\text { Remains }\end{array}$ & $\begin{array}{l}\text { Parasitic } \\
\text { Animals }\end{array}$ & Crústacea & $\begin{array}{l}\text { Other } \\
\text { Animals }\end{array}$ \\
\hline $\begin{array}{l}19^{\circ} 02^{\circ} \mathrm{N} \\
165^{\circ} 36^{\prime} \mathrm{W}\end{array}$ & $\begin{array}{l}\text { Apr } 11 \\
21 \\
65\end{array}$ & $\begin{array}{l}\text { Pacific } \\
\text { Ocean }\end{array}$ & & & 1 & + & & & \\
\hline
\end{tabular}

Parasitic Animals:

Notes on Squid:

Lots: 2 fish

fish remains

Notes on Fish:

Head digested.

General Notes:

PACIFIC OCEANOGRAPHIC BIOLOGICAL SURVEY PROGRAM

SMITHSONIAN OCEANOGRAPHIC SORTING CENTER—SMITHSONIAN INSTITUTION

SI.USNM-E30

$7 \cdot 05$

\begin{tabular}{l|l|} 
Sta. No. & 1034 \\
\hline \begin{tabular}{c|c} 
Lat \& \\
Long.
\end{tabular} & Date \\
\hline $\begin{array}{ll}19^{\circ} 06^{\prime} \mathrm{N} & \text { April } \\
165^{\circ} 23^{\prime} \mathrm{W} & \frac{21}{65}\end{array}$ \\
\hline
\end{tabular}

Species

Puffinus pacificus

SOSC REF. NO. 229

Parasitic Animals:

POBSP. NO. 10

\begin{tabular}{|c|c|c|c|c|c|c|c|}
\hline $\begin{array}{l}\text { Time } \\
\text { Caught }\end{array}$ & Squid & $\begin{array}{l}\text { Squid } \\
\text { Beaks }\end{array}$ & Fish & $\begin{array}{c}\text { Fish } \\
\text { Remains }\end{array}$ & $\begin{array}{l}\text { Parasitic } \\
\text { Animals }\end{array}$ & Crustacea & $\begin{array}{c}\text { Other } \\
\text { Animals }\end{array}$ \\
\hline $\begin{array}{l}\text { Pacific } \\
\text { Ocean }\end{array}$ & 1 & 4 & & - & $\begin{array}{r}\text { Nematodes } \\
\text { (1) }\end{array}$ & & \\
\hline
\end{tabular}

lots: 5 squid

Notes on Squid: 1. Squid in good condition; head and fins attached.

2. Squid remains with a few fish bones.

squid remains

eyeballs (2)

nematode

Notes on Fish:

squid beaks

General Notes:

PACIFIC OCEANOGRAPHIC BIOLOGICAL SURVEY PROGRAM

SMITHSONIAN OCEANOGRAPHIC SORTING CENTER—SMITHSONIAN INSTITUTION

S1. USNM-830

P. Grisham 9/12/66 
SOSC REF. NO. 229

\begin{tabular}{|c|c|c|c|c|c|c|c|c|c|}
\hline Sta. No. & 2035 & & Species & Freg & a $\mathrm{mi}$ & & & NO. Is & \\
\hline $\begin{array}{l}\text { Lat \& } \\
\text { Long. }\end{array}$ & Date & $\begin{array}{l}\text { Time } \\
\text { Caught }\end{array}$ & Squid & $\begin{array}{l}\text { Squid } \\
\text { Beaks }\end{array}$ & Fish & $\begin{array}{c}\text { Fish } \\
\text { Remains }\end{array}$ & $\begin{array}{l}\text { Parasitic } \\
\text { Animals }\end{array}$ & Crustacea & $\begin{array}{l}\text { Other } \\
\text { Animals }\end{array}$ \\
\hline $\begin{array}{l}19^{\circ} 06^{\prime} \mathrm{N} \\
265^{\circ} 23^{\prime} \mathrm{W}\end{array}$ & $\begin{array}{l}\text { April } \\
21, \\
65\end{array}$ & $\begin{array}{l}\text { Paćific } \\
\text { Ocean }\end{array}$ & & & & + & & & \\
\hline
\end{tabular}

Parasitic Animals:

lots: I fish remains

Notes on Squid:

Notes on Fish:

Fish remains with bones.

General Notes:

PACIFIC OCEANOGRAPHIC BIOLOGICAL SURVEY PROGRAM

SMITHSONIAN OCEANOGRAPHIC SORTING CENTER_-SMITHSONIAN INSTITUTION

$\underset{7-65}{5 i-\text { USNM-e30 }}$

P. Grisham 9/12/66

Sta. No.

1036

Species

Puffinus pacificus

SOSC REF. NO.

229

POBSP. NO. 10

\begin{tabular}{|c|c|c|c|c|c|c|c|c|c|}
\hline $\begin{array}{l}\text { Lat \& } \\
\text { Long. }\end{array}$ & Date & $\begin{array}{l}\text { Time } \\
\text { Caught }\end{array}$ & Squid & $\begin{array}{l}\text { Squid } \\
\text { Beaks }\end{array}$ & Fish & $\begin{array}{c}\text { Fish } \\
\text { Remains }\end{array}$ & $\begin{array}{l}\text { Parasitic } \\
\text { Animals }\end{array}$ & Crustacea & $\begin{array}{l}\text { Other } \\
\text { Animals }\end{array}$ \\
\hline $\begin{array}{l}19^{\circ} 44^{\prime} \mathrm{N} \\
163^{\circ} 13^{\circ} \mathrm{W}\end{array}$ & $\begin{array}{l}\text { April } \\
22, \\
65\end{array}$ & $\begin{array}{l}\text { Pacific } \\
\text { Ocean }\end{array}$ & & 71 & & - & $\begin{array}{r}\text { Nematode } \\
\text { (1) }\end{array}$ & & \\
\hline
\end{tabular}

Parasitic Animals:

Notes on Squid:

lots: 3 squid beaks

digested remains

nematode

Notes on Fish:

General Notes:

PACIFIC OCEANOGRAPHIC BIOLOGICAL SURVEY PROGRAM SMITHSONIAN OCEANOGRAPHIC SORTING CENTER—SMITHSONIAN INSTITUTION 
SOSC REF. NO. 229

\begin{tabular}{|c|c|c|c|c|c|c|c|c|c|}
\hline Sta. No. & 1037 & & Specic & & $a \mathrm{da}$ & latra & & NO. & \\
\hline $\begin{array}{l}\text { Lat \& } \\
\text { Long. }\end{array}$ & Date & $\begin{array}{l}\text { Time } \\
\text { Caught }\end{array}$ & Squid & $\begin{array}{l}\text { Squid } \\
\text { Beaks }\end{array}$ & Fish & $\begin{array}{c}\text { Fish } \\
\text { Remains }\end{array}$ & $\begin{array}{l}\text { Parasitic } \\
\text { Animals }\end{array}$ & Crustacea & $\begin{array}{l}\text { Other } \\
\text { Animals }\end{array}$ \\
\hline $\begin{array}{l}10^{\circ} 45^{\prime} \mathrm{N} \\
163^{\circ} \mathrm{II} \mathrm{W}\end{array}$ & $\begin{array}{l}\text { April } \\
22, \\
65\end{array}$ & $\begin{array}{l}\text { Pàcific } \\
\text { Ocean }\end{array}$ & & & & + & & & \\
\hline
\end{tabular}

Parasitic Animals:

lots: 1 fish remains

Notes on Squid:

Notes on Fish:

Fish remains with half of a fish and fish heads.

General Notes:

PACIFIC OCEANOGRAPHIC BIOLOGICAL SURVEY PROGRAM

SMITHSONIAN OCEANOGRAPHIC SORTING CENTER — SMITHSONIAN INSTITUTION

P. Grj.sham 9/13/66

S1-USNM-830

$\operatorname{lin}_{7-85}$

SOSC REF. NO. 229

\begin{tabular}{|c|c|c|c|c|c|c|c|c|c|}
\hline Sta. No. & & & Specie & St & $\mathrm{a} f u$ & ta & & NO. & \\
\hline $\begin{array}{l}\text { Lat \& } \\
\text { Long. }\end{array}$ & Date & $\begin{array}{c}\text { Time } \\
\text { Caught }\end{array}$ & Squid & $\begin{array}{l}\text { Squid } \\
\text { Beaks }\end{array}$ & Fish & $\begin{array}{c}\text { Fish } \\
\text { Remains }\end{array}$ & $\begin{array}{l}\text { Parasitic } \\
\text { Animals }\end{array}$ & Crustacea & $\begin{array}{c}\text { Other } \\
\text { Animals }\end{array}$ \\
\hline $\begin{array}{l}19^{\circ} 4^{\circ} 1 \mathrm{~N} \\
163^{\circ} 02^{\prime} \mathrm{W}\end{array}$ & $\begin{array}{l}\text { April } \\
22, \\
65\end{array}$ & $\begin{array}{l}\text { Pacific } \\
\text { Ocean }\end{array}$ & & & & + & & & \\
\hline
\end{tabular}

Parasitic Animals:

lots: I fish remains

Notes on Squid:

Notes on Fish:

Fish remains with bones.

General Notes:

PACIFIC OCEANOGRAPHIC BIOLOGICAL SURVEY PROGRAM

SMITHSONIAN OCEANOGRAPHIC SORTING CENTER—SMITHSONIAN INSTITUTION

P. Grisham 8/16/66 
Sta. No. 1039

\begin{tabular}{|c|c|c|}
\hline $\begin{array}{l}\text { Lat \& } \\
\text { Long. }\end{array}$ & Date & $\begin{array}{c}\text { Time } \\
\text { Caught }\end{array}$ \\
\hline $\begin{array}{l}19^{\circ} 52^{\prime} \mathrm{N} \\
162^{\circ} 49^{\prime} \mathrm{W}\end{array}$ & $\begin{array}{l}\text { April } \\
22, \\
65\end{array}$ & $\begin{array}{l}\text { Pačific } \\
\text { Ocean }\end{array}$ \\
\hline
\end{tabular}

Parasitic Animals:
SOSC REF. NO. 229

POBSP. NO. 20

\section{Notes on Squid:}

lots: 4 digested remains squid beaks halobates eyeballs (5)

\section{Notes on Fish:}

\begin{tabular}{|c|c|c|c|c|c|c|}
\hline Squid & $\begin{array}{l}\text { Squid } \\
\text { Beaks }\end{array}$ & Fish & $\begin{array}{c}\text { Fish } \\
\text { Remains }\end{array}$ & $\begin{array}{l}\text { Parasitic } \\
\text { Animals }\end{array}$ & Crustacea & $\begin{array}{c}\text { Other } \\
\text { Animals }\end{array}$ \\
\hline & 4 & & - & & & $\begin{array}{c}\text { Halobates } \\
\text { (3) }\end{array}$ \\
\hline
\end{tabular}

\section{( Notes on Fish: \\ General Notes: 1. Digested remains with bits of wood and halobate fragments. \\ 2. Eyeballs are very large. \\ 3. Few halobates with broken legs. \\ PACIFIC OCEANOGRAPHIC BIOLOGICAL SURVEY PROGRAM \\ SMITHSONIAN OCEANOGRAPHIC SORTING CENTER—SMITHSONIAN INSTITUTION \\ SI.USNM-930 \\ P. Grisham 9/12/66 \\ $7-65$}

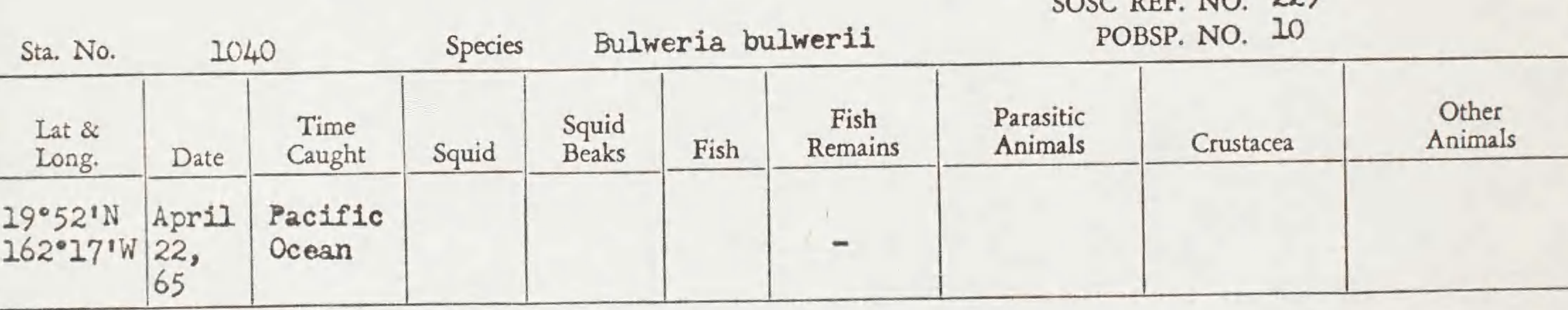

Parasitic Animals:

lots: 2 eyeballs (3)

Notes on Squid:

digested remains

Notes on Fish:

General Notes:

Digested remains beyond identification.

PACIFIC OCEANOGRAPHIC BIOLOGICAL SURVEY PROGRAM

SMITHSONIAN OCEANOGRAPHIC SORTING CENTER—SMITHSONIAN INSTITUTION 
SOSC REF. NO. 229

Sta. No.

Species

Sterna fuscata

POBSP. NO. 10

\begin{tabular}{|c|c|c|c|c|c|c|c|c|c|}
\hline $\begin{array}{l}\text { Lat \& } \\
\text { Long. }\end{array}$ & Date & $\begin{array}{c}\text { Time } \\
\text { Caught }\end{array}$ & Squid & $\begin{array}{l}\text { Squid } \\
\text { Beaks }\end{array}$ & Fish & $\begin{array}{c}\text { Fish } \\
\text { Remains }\end{array}$ & $\begin{array}{l}\text { Parasitic } \\
\text { Animals }\end{array}$ & Crustacea & $\begin{array}{l}\text { Other } \\
\text { Animals }\end{array}$ \\
\hline $\begin{array}{l}19^{\circ} 52^{\prime} \mathrm{N} \\
162^{\circ} 47^{\prime} \mathrm{W}\end{array}$ & $\begin{array}{l}\text { April } \\
22, \\
65\end{array}$ & $\begin{array}{l}\text { Pacific } \\
\text { Ocean }\end{array}$ & & & & + & & & \\
\hline
\end{tabular}

Parasitic Animals:

10ts: 1 fish remains

Notes on Squid:

Notes on Fish:

Fish remains with half of a fish.

General Notes:

PACIFIC OCEANOGRAPHIC BIOLOGICAL SURVEY PROGRAM SMITHSONIAN OCEANOGRAPHIC SORTING CENTER—SMITHSONIAN INSTITUTION

SI-USNA- 30

P. Grisham 8/23/66

Y

Sta. No.

Species Sterna fuscata

SOSC REF. NO. 229

POBSP. NO. 10

\begin{tabular}{|c|c|c|c|c|c|c|c|c|c|}
\hline $\begin{array}{l}\text { Lat \& } \\
\text { Long. }\end{array}$ & Date & $\begin{array}{l}\text { Time } \\
\text { Caught }\end{array}$ & Squid & $\begin{array}{l}\text { Squid } \\
\text { Beaks }\end{array}$ & Fish & $\begin{array}{c}\text { Fish } \\
\text { Remains }\end{array}$ & $\begin{array}{l}\text { Parasitic } \\
\text { Animals }\end{array}$ & Crustacea & $\begin{array}{c}\text { Other } \\
\text { Animals }\end{array}$ \\
\hline $\begin{array}{l}19^{\circ} 52^{\prime} \mathrm{N} \\
162^{\circ} 47^{\prime} \mathrm{W}\end{array}$ & $\begin{array}{l}\text { Apri] } \\
22, \\
65\end{array}$ & $\begin{array}{l}\text { Pacific } \\
\text { Ocean }\end{array}$ & & & & - & & & \\
\hline
\end{tabular}

Parasitic Animals:

lots: 1 digested remains

Notes on Squid:

Notes on Fish:

General Notes:

Digested remains beyond identification.

PACIFIC OCEANOGRAPHIC BIOLOGICAL SURVEY PROGRAM SMITHSONIAN OCEANOGRAPHIC SORTING CENTER SMITHSONIAN INSTITUTION

P. Grisham $8 / 16 / 66$ 
SOSC REF. NO. 229

Sta. No. $\quad 1043$

Species Sterna fuscata

POBSP. NO. 10

\begin{tabular}{|c|c|c|c|c|c|c|c|c|c|}
\hline $\begin{array}{l}\text { Lat \& } \\
\text { Long. }\end{array}$ & Date & $\begin{array}{l}\text { Time } \\
\text { Caught }\end{array}$ & Squid & $\begin{array}{l}\text { Squid } \\
\text { Beaks }\end{array}$ & Fish & $\begin{array}{c}\text { Fish } \\
\text { Remains }\end{array}$ & $\begin{array}{l}\text { Parasitic } \\
\text { Animals }\end{array}$ & Crustacea & $\begin{array}{l}\text { Other } \\
\text { Animals }\end{array}$ \\
\hline $\begin{array}{l}19^{\circ} 52^{\prime} \mathrm{N} \\
162^{\circ} 47^{\prime} \mathrm{W}\end{array}$ & $\begin{array}{l}\text { Apriz } \\
22, \\
65\end{array}$ & $\begin{array}{l}\text { Pacific } \\
\text { Ocean }\end{array}$ & & & & - & & & \\
\hline
\end{tabular}

Parasitic Animals:

Lots: 1 digested remains

Notes on Squid:

Notes on Fish:

General Notes:

Digested remains beyond identification.

PACIFIC OCEANOGRAPHIC BIOLOGICAL SURVEY PROGRAM

SMITHSONIAN OCEANOGRAPHIC SORTING CENTER—SMITHSONIAN INSTITUTION

P. Grisham 8/15/66

S1-U8NM-830

7-68

$$
3
$$

SOSC REF. NO. 229

\begin{tabular}{|c|c|c|c|c|c|c|c|c|c|}
\hline \multirow{2}{*}{$\begin{array}{l}\text { Sta. No. } \\
\text { Lat \& } \\
\text { Long. }\end{array}$} & 1044 & \multicolumn{2}{|c|}{ Species } & \multicolumn{3}{|c|}{ Sterna fuscata } & \multicolumn{2}{|c|}{ POBSP. NO. 10} & \multirow[b]{2}{*}{$\begin{array}{c}\text { Other } \\
\text { Animals }\end{array}$} \\
\hline & Date & $\begin{array}{l}\text { Time } \\
\text { Caught }\end{array}$ & Squid & $\begin{array}{l}\text { Squid } \\
\text { Beaks }\end{array}$ & Fish & $\begin{array}{c}\text { Fish } \\
\text { Remains }\end{array}$ & $\begin{array}{l}\text { Parasitic } \\
\text { Animals }\end{array}$ & Crustacea & \\
\hline $\begin{array}{l}19^{\circ} 55^{\prime} \mathrm{N} \\
162^{\circ} 40^{\prime} \mathrm{W}\end{array}$ & $\begin{array}{l}\text { April } \\
22, \\
65\end{array}$ & $\begin{array}{l}\text { Pacific } \\
\text { Ocean }\end{array}$ & & 4 & & - & & & \\
\hline
\end{tabular}

Parasitic Animals:

lots: 2 squid beaks

Notes on Squid: digested remains

Notes on Fish:

General Notes:

Digested remains beyond identification.

PACIFIC OCEANOGRAPHIC BIOLOGICAL SURVEY PROGRAM

SMITHSONIAN OCEANOGRAPHIC SORTING CENTER_SMITHSONIAN INSTITUTION

S1-USmM-830

P. Grisham 9/12/66 
SOSC REF. NO. 229

\begin{tabular}{c|c|} 
Sta. No. & 1045 \\
\hline \begin{tabular}{c|c} 
Lat \& \\
Long.
\end{tabular} & Date \\
\hline $20^{\circ} 00^{\prime} \mathrm{N}$ & $\begin{array}{l}\text { April } \\
162^{\circ} 19^{\prime} \mathrm{W}\end{array}$ \\
$\begin{array}{ll}22, \\
65\end{array}$ \\
\hline
\end{tabular}

Species Bulweria bulweri1

POBSP. NO. 10

Parasitic Animals:

Iots: 3 squid beaks

Notes on Squid:

eyeballs (2)

digested remains

Notes on Fish:

General Notes:

PACIFIC OCEANOGRAPHIC BIOLOGICAL SURVEY PROGRAM SMITHSONIAN OCEANOGRAPHIC SORTING CENTER—SMITHSONIAN INSTITUTION

S1-USAM-830

P. Grisham 9/6/66

7-08

$\ddot{z}$

SOSC REF. NO. 229

Sta. No. 1046 Species Bulweria bulwerii

POBSP. NO. 10

\begin{tabular}{|c|c|c|c|c|c|c|c|c|c|}
\hline $\begin{array}{l}\text { Lat \& } \\
\text { Long. }\end{array}$ & Date & $\begin{array}{l}\text { Time } \\
\text { Caught }\end{array}$ & Squid & $\begin{array}{l}\text { Squid } \\
\text { Beaks }\end{array}$ & Fish & $\begin{array}{c}\text { Fish } \\
\text { Remains }\end{array}$ & $\begin{array}{l}\text { Parasitic } \\
\text { Animals }\end{array}$ & Crustacea & $\begin{array}{c}\text { Other } \\
\text { Animals }\end{array}$ \\
\hline $\begin{array}{l}20^{\circ} 02^{\prime} \mathrm{N} \\
162^{\circ} 09^{\prime} \mathrm{W}\end{array}$ & $\begin{array}{l}\text { April } \\
22, \\
65\end{array}$ & $\begin{array}{l}\text { Pacific } \\
\text { Ocean }\end{array}$ & & & & - & & & \\
\hline
\end{tabular}

Parasitic Animals:

Lots: 1 digested remains

Notes on Squid:

Notes on Fish:

General Notes:

Digested remains beyond identification.

PACIFIC OCEANOGRAPHIC BIOLOGICAL SURVEY PROGRAM

SMITHSONIAN OCEANOGRAPHIC SORTING CENTER_-SMITHSONIAN INSTITUTION

S1-USHM-630

P. Grisham 9/6/66 


\begin{tabular}{|c|c|c|c|c|c|c|c|c|c|}
\hline Sta. No. & 10 & 47 & Specie & Bul & $1 \mathrm{a} b$ & eri1 & & $\begin{array}{l}\text { NO. } \\
\text { NO. }\end{array}$ & \\
\hline $\begin{array}{l}\text { Lat \& } \\
\text { Long. }\end{array}$ & Date & $\begin{array}{l}\text { Time } \\
\text { Caught }\end{array}$ & Squid & $\begin{array}{l}\text { Squid } \\
\text { Beaks }\end{array}$ & Fish & $\begin{array}{c}\text { Fish } \\
\text { Remains }\end{array}$ & $\begin{array}{l}\text { Parasitic } \\
\text { Animals }\end{array}$ & Crustacea & $\begin{array}{c}\text { Other } \\
\text { Animals }\end{array}$ \\
\hline $\begin{array}{l}20^{\circ} 03^{\prime} \mathrm{N} \\
162^{\circ} 05^{\prime} \mathrm{W}\end{array}$ & $\begin{array}{l}\text { Apri1 } \\
22, \\
65\end{array}$ & $\begin{array}{l}\text { Pacific } \\
\text { Ocean }\end{array}$ & & & & - & & & \\
\hline
\end{tabular}

Parasitic Animals:

lots: 1 digested remains

Notes on Squid:

Notes on Fish:

General Notes:

'Digested remains beyond identification.

PACIFIC OCEANOGRAPHIC BIOLOGICAL SURVEY PROGRAM SMITHSONIAN OCEANOGRAPHIC SORTING CENTER—SMITHSONIAN INSTITUTION

P. Grisham 8/16/66

SI-USNM-830

Sta. No. $\quad 2048$

\begin{tabular}{|c|c|c|c|c|c|c|c|c|c|}
\hline $\begin{array}{l}\text { Lat \& } \\
\text { Long. }\end{array}$ & Date & $\begin{array}{l}\text { Time } \\
\text { Caught }\end{array}$ & Squid & $\begin{array}{l}\text { Squid } \\
\text { Beaks }\end{array}$ & Fish & $\begin{array}{c}\text { Fish } \\
\text { Remains }\end{array}$ & $\begin{array}{l}\text { Parasitic } \\
\text { Animals }\end{array}$ & Crustacea & $\begin{array}{l}\text { Other } \\
\text { Animals }\end{array}$ \\
\hline $\begin{array}{l}20^{\circ} 06^{\prime} \mathrm{N} \\
161^{\circ} 52^{\prime} \mathrm{W}\end{array}$ & $\begin{array}{l}\text { April } \\
22, \\
65\end{array}$ & $\begin{array}{l}\text { Pacific } \\
\text { Ocean }\end{array}$ & & 2 & & - & & & \\
\hline
\end{tabular}

Parasitic Animals:

lots: 2 squid beaks

Notes on Squid:

Squid remains with tentacles; suckers are visible. squid remains

Notes on Fish:

General Notes:

PACIFIC OCEANOGRAPHIC BIOLOGICAL SURVEY PROGRAM SMITHSONIAN OCEANOGRAPHIC SORTING CENTER—SMITHSONIAN INSTITUTION 


\begin{tabular}{|c|c|c|c|c|c|c|c|c|c|}
\hline Sta. No. & 1049 & & Speci & & ria & wer11 & & $\begin{array}{l}\text { No. } \\
\text { No. }\end{array}$ & \\
\hline $\begin{array}{l}\text { Lat \& } \\
\text { Long. }\end{array}$ & Date & $\begin{array}{c}\text { Time } \\
\text { Caught }\end{array}$ & Squid & $\begin{array}{l}\text { Squid } \\
\text { Beaks }\end{array}$ & Fish & $\begin{array}{c}\text { Fish } \\
\text { Remains }\end{array}$ & $\begin{array}{l}\text { Parasitic } \\
\text { Animals }\end{array}$ & Crustacea & $\begin{array}{c}\text { Other } \\
\text { Animals }\end{array}$ \\
\hline $\begin{array}{l}20^{\circ} 08^{\prime} \mathrm{N} \\
161^{\circ} 47^{\prime} \mathrm{W}\end{array}$ & $\begin{array}{l}\text { April } \\
22, \\
65\end{array}$ & $\begin{array}{l}\text { Paclfic } \\
\text { Ocean }\end{array}$ & & 2 & & - & & & \\
\hline
\end{tabular}

Parasitic Animals:

lots: 2 squid beaks

Notes on Squid:

digested remains

Notes on Fish:

General Notes:

Digested remains with halobate fragments.

PACIFIC OCEANOGRAPHIC BIOLOGICAL SURVEY PROGRAM

SMITHSONIAN OCEANOGRAPHIC SORTING CENTER—SMITHSONIAN INSTITUTION

S1-USNM- 30

P. Grisham $8 / 23 / 66$

$\underset{7 \rightarrow 65}{51-45}$

5

SOSC REF. NO. 229

POBSP. NO. 10

\begin{tabular}{|c|c|c|c|c|c|c|}
\hline Sta. No. & \multicolumn{2}{|c|}{1050} & Specie & \multicolumn{3}{|c|}{ Sula sula } \\
\hline $\begin{array}{l}\text { Lat \& } \\
\text { Long. }\end{array}$ & Date & $\begin{array}{l}\text { Time } \\
\text { Caught }\end{array}$ & Squid & $\begin{array}{l}\text { Squid } \\
\text { Beaks }\end{array}$ & Fish & $\mathrm{Re}$ \\
\hline $\begin{array}{l}20^{\circ} 13^{\prime} \mathrm{N} \\
161^{\circ} 20^{\prime} \mathrm{W}\end{array}$ & $\begin{array}{l}\text { April } \\
22, \\
65,\end{array}$ & $\begin{array}{l}\text { Pacific } \\
\text { Ocean }\end{array}$ & & 3 & 1 & \\
\hline \multicolumn{7}{|c|}{ Parasitic Animals: } \\
\hline \multicolumn{7}{|c|}{ Notes on Squid: } \\
\hline Notes on & Fish: & $\begin{array}{l}\text { 1. Hea } \\
\text { 2. Fis }\end{array}$ & $\begin{array}{l}\text { diges } \\
\text { rema: }\end{array}$ & $\begin{array}{l}\text {; po } \\
\text { with }\end{array}$ & & \\
\hline
\end{tabular}

General Notes:

lots: 3 fish

fish remains

squid beaks

Other

Animals

PACIFIC OCEANOGRAPHIC BIOLOGICAL SURVEY PROGRAM

PACIFIC OCEANOGRAPHIC BIOLOGICAL SURVEY PMITHSONIAN OCEANOGRAPHIC SORTING CENTER-SMITHSONIAN INSTITUTION
P. Grisham $9 / 14 / 66$ 
SOSC REF. NO. 229

Sta. No.

Species Sula dactylatra

POBSP. NO. 10

\begin{tabular}{|c|c|c|c|c|c|c|c|c|c|}
\hline $\begin{array}{l}\text { Lat \& } \\
\text { Long. }\end{array}$ & Date & $\begin{array}{c}\text { Time } \\
\text { Caught }\end{array}$ & Squid & $\begin{array}{l}\text { Squid } \\
\text { Beaks }\end{array}$ & Fish & $\begin{array}{c}\text { Fish } \\
\text { Remains }\end{array}$ & $\begin{array}{l}\text { Parasitic } \\
\text { Animals }\end{array}$ & Crustacea & $\begin{array}{l}\text { Other } \\
\text { Animals }\end{array}$ \\
\hline $\begin{array}{l}18^{\circ} 43^{\prime} \mathrm{N} \\
170^{\circ} 31^{\prime} \mathrm{W}\end{array}$ & $\begin{array}{l}\text { May } \\
21, \\
65\end{array}$ & $\begin{array}{l}\text { Pacific } \\
\text { Ocean }\end{array}$ & & & & + & & & \\
\hline
\end{tabular}

Parasitic Animals:

lots: 1 fish remains

Notes on Squid:

Notes on Fish:

Fish remains with half of a fish and fish bones.

General Notes:

PACIFIC OCEANOGRAPHIC BIOLOGICAL SURVEY PROGRAM SMITHSONIAN OCEANOGRAPHIC SORTING CENTER — SMITHSONIAN INSTITUTION

S1-USNM-830 P. Grisham 8/29/66

T1-6s F

Sta. No.

1251

Species

Diomedea nigripes

SOSC REF. NO. 229

POBSP. NO. 10

\begin{tabular}{|c|c|c|c|c|c|c|c|c|c|}
\hline $\begin{array}{l}\text { Lat \& } \\
\text { Long. }\end{array}$ & Date & $\begin{array}{l}\text { Time } \\
\text { Caught }\end{array}$ & Squid & $\begin{array}{l}\text { Squid } \\
\text { Beaks }\end{array}$ & Fish & $\begin{array}{l}\text { Fish } \\
\text { Remains }\end{array}$ & $\begin{array}{l}\text { Parasitic } \\
\text { Animals }\end{array}$ & Crustacea & $\begin{array}{c}\text { Other } \\
\text { Animals }\end{array}$ \\
\hline $\begin{array}{l}21 \cdot 20^{\prime} \mathrm{N} \\
167^{\circ} 41^{\prime} \mathrm{W}\end{array}$ & $\begin{array}{l}\text { May } \\
22, \\
65\end{array}$ & $\begin{array}{l}\text { Pacific } \\
\text { Ocean }\end{array}$ & & & & - & $\begin{array}{l}\text { Nematodes } \\
\text { (6) }\end{array}$ & & 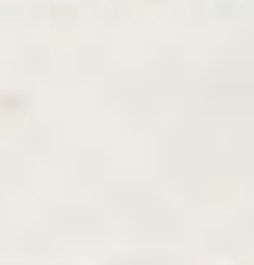 \\
\hline
\end{tabular}

Parasitic Animals:

Notes on Squid:

lats: 3 nematodes

digested remains

Notes on Fish:

General Notes:

PACIFIC OCEANOGRAPHIC BIOLOGICAL SURVEY PROGRAM

SMITHSONIAN OCEANOGRAPHIC SORTING CENTER—SMITHSONIAN INSTITUTION

P. Grisham

$8 / 29 / 66$ 
SOSC REF. NO. 229

Sta. No. 1152

Species Diomedea nigripes

POBSP. NO. 10

\begin{tabular}{c|c|c|c|c|c|c|c|c}
\hline $\begin{array}{c}\text { Lat \& } \\
\text { Long. }\end{array}$ & Date & $\begin{array}{c}\text { Time } \\
\text { Caught }\end{array}$ & Squid & $\begin{array}{c}\text { Squid } \\
\text { Beaks }\end{array}$ & Fish & $\begin{array}{c}\text { Fish } \\
\text { Remains }\end{array}$ & $\begin{array}{c}\text { Parasitic } \\
\text { Animals }\end{array}$ & $\begin{array}{c}\text { Other } \\
\text { Animals }\end{array}$ \\
\hline $\begin{array}{l}27^{\circ} 23^{\prime} \mathrm{N} \\
167^{\circ} 37^{\prime} \mathrm{W}\end{array}$ & $\begin{array}{l}\text { May } \\
22, \\
65\end{array}$ & $\begin{array}{l}\text { Pacific } \\
\text { Ocean }\end{array}$ & & 9 & + & $\begin{array}{c}\text { Cephalacea } \\
\text { eggs }\end{array}$ & \\
\hline
\end{tabular}

Parasitic Animals:

Lots: fish remains

Notes on Squid: cephalopod eggs squid beaks

Notes on Fish:

General Notes:

PACIFIC OCEANOGRAPHIC BIOLOGICAL SURVEY PROGRAM SMITHSONIAN OCEANOGRAPHIC SORTING CENTER_SMITHSONIAN INSTITUTION

S1.USNM-830 P. Grisham 8/17/66

$\dddot{y}$

SOSC REF. NO. 229

Sta. No. 1214 Species Sula dactylatra POBSP. NO. 10

\begin{tabular}{|c|c|c|c|c|c|c|c|c|c|}
\hline $\begin{array}{l}\text { Lat \& } \\
\text { Long. }\end{array}$ & Date & $\begin{array}{l}\text { Time } \\
\text { Caught }\end{array}$ & Squid & $\begin{array}{l}\text { Squid } \\
\text { Beaks }\end{array}$ & Fish & $\begin{array}{c}\text { Fish } \\
\text { Remains }\end{array}$ & $\begin{array}{l}\text { Parasitic } \\
\text { Animals }\end{array}$ & Crustacea & $\begin{array}{c}\text { Other } \\
\text { Animals }\end{array}$ \\
\hline $\begin{array}{l}07^{\circ} 02^{\prime} \mathrm{N} \\
152^{\circ} 27^{1} \mathrm{~N}\end{array}$ & $\begin{array}{l}\text { June } \\
10, \\
65\end{array}$ & $\begin{array}{l}\text { Pacific } \\
\text { Ocean }\end{array}$ & & 2 & & - & & & \\
\hline
\end{tabular}

Parasitic Animals:

Notes on Squid:

lots: 2 squid beaks digested remains

Notes on Fish:

General Notes:

PACIFIC OCEANOGRAPHIC BIOLOGICAL SURVEY PROGRAM SMITHSONIAN OCEANOGRAPHIC SORTING CENTER—SMITHSONIAN INSTITUTION 
SOSC REF. NO. 229

\begin{tabular}{|c|c|c|c|c|c|c|c|c|c|}
\hline $\begin{array}{l}\text { Lat \& } \\
\text { Long. }\end{array}$ & Date & $\begin{array}{l}\text { Time } \\
\text { Caught }\end{array}$ & Squid & $\begin{array}{l}\text { Squid } \\
\text { Beaks }\end{array}$ & Fish & $\begin{array}{c}\text { Fish } \\
\text { Remains }\end{array}$ & $\begin{array}{l}\text { Parasitic } \\
\text { Animals }\end{array}$ & Crustacea & $\begin{array}{l}\text { Other } \\
\text { Animals }\end{array}$ \\
\hline $\begin{array}{l}07^{\circ} 02^{\prime} \mathrm{N} \\
152^{\circ} 28^{\prime} \mathrm{W}\end{array}$ & $\begin{array}{l}\text { June } \\
10, \\
65\end{array}$ & $\begin{array}{l}\text { Pacific } \\
\text { Ocean }\end{array}$ & & & 2 & + & & & \\
\hline
\end{tabular}

Parasitic Animals:

lots: 2 fish remains

Notes on Squid: fish

$\begin{array}{ll}\text { Notes on Fish: } & \text { 1. Fish remains with bones. } \\ & \text { 2. Heads digested. }\end{array}$

General Notes:

PACIFIC OCEANOGRAPHIC BIOLOGICAL SURVEY PROGRAM SMITHSONIAN OCEANOGRAPHIC SORTING CENTER—SMITHSONIAN INSTITUTION

SI-USNM- $\rightarrow 30$ T-6s P. Grisham $8 / 22 / 66$ z

\begin{tabular}{|c|c|c|c|c|c|c|c|c|c|}
\hline Sta. No. & 123 & & Species & Sula & dacty & $r^{2}$ & & $\begin{array}{l}\text { NO. } \\
\text { NO. }\end{array}$ & \\
\hline $\begin{array}{l}\text { Lat \& } \\
\text { Long. }\end{array}$ & Date & $\begin{array}{l}\text { Time } \\
\text { Caught }\end{array}$ & Squid & $\begin{array}{l}\text { Squid } \\
\text { Beaks }\end{array}$ & Fish & $\begin{array}{c}\text { Fish } \\
\text { Remains } \\
\end{array}$ & $\begin{array}{l}\text { Parasitic } \\
\text { Animals }\end{array}$ & Crustacea & $\begin{array}{c}\text { Other } \\
\text { Animals }\end{array}$ \\
\hline $\begin{array}{l}06^{\circ} 20^{\prime N} \\
153^{\circ} 14^{\prime} \mathrm{W}\end{array}$ & $\begin{array}{l}\text { Sept. } \\
10, \\
65\end{array}$ & $\begin{array}{l}\text { Pacifid } \\
\text { Ocean }\end{array}$ & & & 3 & 4 & & & \\
\hline
\end{tabular}

Parasitic Animals:

lots: 2 Iish

Notes on Squid:

fish remains

Notes on Fish:

Heads of two digested.

General Notes:

No, stomach, only contents.

PACIFIC OCEANOGRAPHIC BIOLOGICAL SURVEY PROGRAM

SMITHSONIAN OCEANOGRAPHIC SORTING CENTER—SMITHSONIAN INSTITUTION 


\begin{tabular}{|c|c|c|c|c|c|c|c|c|c|}
\hline Sta. No. & & & Specie & Sula & cty. & & & $\begin{array}{l}\text { NO. } \\
\text { NO. }\end{array}$ & \\
\hline $\begin{array}{l}\text { Lat \& } \\
\text { Long. }\end{array}$ & Date & $\begin{array}{c}\text { Time } \\
\text { Caught }\end{array}$ & Squid & $\begin{array}{l}\text { Squid } \\
\text { Beaks }\end{array}$ & Fish & $\begin{array}{c}\text { Fish } \\
\text { Remains }\end{array}$ & $\begin{array}{l}\text { Parasitic } \\
\text { Animals }\end{array}$ & Crustacea & $\begin{array}{l}\text { Other } \\
\text { Animals }\end{array}$ \\
\hline $\begin{array}{l}06^{\circ} 13^{\prime} \mathrm{N} \\
153^{\circ} 34^{\prime} \mathrm{W}\end{array}$ & $\begin{array}{l}\text { June } \\
10, \\
65\end{array}$ & $\begin{array}{l}\text { Pacific } \\
\text { Ocosn }\end{array}$ & & & & + & & & \\
\hline
\end{tabular}

Parasitic Animals:

Iots: I fish remains

Notes on Squid:

Notes on Fish:

General Notes:

NO stomach, only stomach contents.

PACIFIC OCEANOGRAPHIC BIOLOGICAL SURVEY PROGRAM

SMITHSONIAN OCEANOGRAPHIC SORTING CENTER—SMITHSONIAN INSTITUTION

Si-USNM-830

$\lim _{7 \rightarrow 68}$

P. Grisham $8 / 22 / 66$

\begin{tabular}{|c|c|c|c|c|c|c|c|c|c|}
\hline Sta. No. & 241 & & Speci & St & 14 & & & $\begin{array}{l}\text { No. } 229 \\
\text { No. } 10\end{array}$ & \\
\hline $\begin{array}{l}\text { Lat \& } \\
\text { Long. }\end{array}$ & Date & $\begin{array}{c}\text { Time } \\
\text { Caught }\end{array}$ & Squid & $\begin{array}{l}\text { Squid } \\
\text { Beaks }\end{array}$ & Fish & $\begin{array}{c}\text { Fish } \\
\text { Remains }\end{array}$ & $\begin{array}{l}\text { Parasitic } \\
\text { Animals }\end{array}$ & Crustacea & $\begin{array}{c}\text { Other } \\
\text { Animals }\end{array}$ \\
\hline $\begin{array}{l}06^{\circ} 11^{\prime} \mathrm{N} \\
155^{\circ} 20^{\prime} \mathrm{W}\end{array}$ & $\begin{array}{l}\text { June } \\
\frac{11}{65},\end{array}$ & $\begin{array}{l}\text { Pacific } \\
\text { Ocean }\end{array}$ & 4 & & & - & & & \\
\hline
\end{tabular}

Parasitic Animals:

Notes on Squid:

Heads of two digested.

Lots: 2 squids squid remains

Notes on Fish:

General Notes:

PACIFIC OCEANOGRAPHIC BIOLOGICAL SURVEY PROGRAM

SMITHSONIAN OCEANOGRAPHIC SORTING CENTER—SMITHSONIAN INSTITUTION

S1-USNM-830

P. Grisham 8/23/66 
SOSC REF. NO. 229

Sta. No.124,2

Species Sula dactylatra

POBSP. NO. 10

\begin{tabular}{c|c|c|c|c|c|c|c|c|c}
\hline $\begin{array}{c}\text { Lat \& } \\
\text { Long. }\end{array}$ & Date & $\begin{array}{c}\text { Time } \\
\text { Caught }\end{array}$ & Squid & $\begin{array}{c}\text { Squid } \\
\text { Beaks }\end{array}$ & Fish & $\begin{array}{c}\text { Fish } \\
\text { Remains }\end{array}$ & $\begin{array}{c}\text { Parasitic } \\
\text { Animals }\end{array}$ & Crustacea \\
\hline $\begin{array}{l}06^{\circ} 2^{\prime} \mathrm{N} \\
155^{\circ} 21^{\prime} \mathrm{W}\end{array}$ & $\begin{array}{l}\text { June } \\
\frac{17}{65}\end{array}$ & $\begin{array}{l}\text { Pacific } \\
\text { Ocean }\end{array}$ & & & 1 & - & & \\
\hline
\end{tabular}

Parasitic Animals:

Notes on Squid:

lats: $1 \mathrm{fish}$

Notes on Fish:

Head attached.

General Notes:

No stomach, only contents.

PACIFIC OCEANOGRAPHIC BIOLOGICAL SURVEY PROGRAM

SMITHSONIAN OCEANOGRAPHIC SORTING CENTER—SMITHSONIAN INSTITUTION

S1-USMM-830

P. Grisham $8 / 4 / 66$

$-3$

SOSC REF. NO. 229

POBSP. NO. 10

\begin{tabular}{|c|c|c|c|c|c|c|c|c|c|}
\hline Sta. No. & 1247 & & Species & Sul & dacty & tra & & NO. & \\
\hline $\begin{array}{l}\text { Lat \& } \\
\text { Long. }\end{array}$ & Date & $\begin{array}{l}\text { Time } \\
\text { Caught }\end{array}$ & Squid & $\begin{array}{l}\text { Squid } \\
\text { Beaks }\end{array}$ & Fish & $\begin{array}{c}\text { Fish } \\
\text { Remains }\end{array}$ & $\begin{array}{l}\text { Parasitic } \\
\text { Animals }\end{array}$ & Crustacea & $\begin{array}{c}\text { Other } \\
\text { Animals }\end{array}$ \\
\hline $\begin{array}{l}6^{\circ} 22^{\prime} \mathrm{N} \\
56^{\circ} 07^{\prime} \mathrm{W}\end{array}$ & $\begin{array}{l}\text { June } \\
\frac{11}{65},\end{array}$ & $\begin{array}{l}\text { Pacific } \\
\text { Ocean }\end{array}$ & & & & + & $\begin{array}{r}\text { Tapeworms } \\
(4)\end{array}$ & & \\
\hline
\end{tabular}

Parasitic Animals:

Notes on Squid:

lots: 3 tapeworms

fish remains

eyeballs (2)

Notes on Fish:

Fish remains with bones.

General Notes:

PACIFIC OCEANOGRAPHIC BIOLOGICAL SURVEY PROGRAM

SMITHSONIAN OCEANOGRAPHIC SORTING CENTER—SMITHSONIAN INSTITUTION

S1.USNM-830

P. Grisham 8/22/66 
SOSC REF. NO. 229

Sta. No. $1256 \quad$ Species Fregata minor POBSP. NO. 10

\begin{tabular}{|c|c|c|c|c|c|c|c|c|c|}
\hline $\begin{array}{l}\text { Lat \& } \\
\text { Long. }\end{array}$ & Date & $\begin{array}{c}\text { Time } \\
\text { Caught }\end{array}$ & Squid & $\begin{array}{l}\text { Squid } \\
\text { Beaks }\end{array}$ & Fish & $\begin{array}{c}\text { Fish } \\
\text { Remains }\end{array}$ & $\begin{array}{l}\text { Parasitic } \\
\text { Animals }\end{array}$ & Crustacea & $\begin{array}{c}\text { Other } \\
\text { Animals }\end{array}$ \\
\hline $\begin{array}{l}07^{\circ} 05^{\prime} \mathrm{N} \\
158^{\circ} 44^{\prime} \mathrm{W}\end{array}$ & $\begin{array}{l}\text { June } \\
12, \\
65\end{array}$ & $\begin{array}{l}\text { Pacific } \\
\text { Ocean }\end{array}$ & & 32 & 2 & + & & & \\
\hline
\end{tabular}

Parasitic Animals:

lots: 4 squid beaks

Notes on Squid: eyeballs (3)

fish

fish remains

Notes on Fish: 1. Fish remains with bones.

2. Good condition.

General Notes:

PACIFIC OCEANOGRAPHIC BIOLOGICAL SURVEY PROGRAM

SMITHSONIAN OCEANOGRAPHIC SORTING CENTER—SMITHSONIAN INSTITUTION

SI-UลNM-830

7-6s

P. Grisham 8/4/66

5

SOSC REF. NO. 229

POBSP. NO. 10

\begin{tabular}{|c|c|c|c|c|c|c|c|c|c|}
\hline \multirow{3}{*}{$\begin{array}{l}\text { Sta. No. } \\
\text { Lat \& } \\
\text { Long. }\end{array}$} & \multirow{2}{*}{\multicolumn{2}{|c|}{1261}} & \multirow{2}{*}{ Species } & \multirow{2}{*}{\multicolumn{3}{|c|}{ Puffinus pacificus }} & \multicolumn{2}{|c|}{ POBSP. NO. 10} & \multirow[b]{3}{*}{$\begin{array}{l}\text { Other } \\
\text { Animals }\end{array}$} \\
\hline & & & & & & & & & \\
\hline & Date & $\begin{array}{c}\text { Time } \\
\text { Caught }\end{array}$ & Squid & $\begin{array}{l}\text { Squid } \\
\text { Beaks }\end{array}$ & Fish & $\begin{array}{c}\text { Fish } \\
\text { Remains }\end{array}$ & $\begin{array}{l}\text { Parasitic } \\
\text { Animals }\end{array}$ & Crustacea & \\
\hline $\begin{array}{l}07^{\circ} 18^{\prime} \mathrm{N} \\
159^{\circ} 30^{\prime} \mathrm{W}\end{array}$ & $\begin{array}{l}\text { June } \\
12, \\
65\end{array}$ & $\begin{array}{l}\text { Pacific } \\
\text { Ocean }\end{array}$ & & 36 & & - & & & \\
\hline
\end{tabular}

Parasitic Animals:

Notes on Squid:

Lots: 2 squid beaks

digested remains

Notes on Fish:

General Notes:

Digested remains beyond identification.

PACIFIC OCEANOGRAPHIC BIOLOGICAL SURVEY PROGRAM

SMITHSONIAN OCEANOGRAPHIC SORTING CENTER_SMITHSONIAN INSTITUTION

S1-USNm-830 


\begin{tabular}{|c|c|c|c|c|c|c|c|c|c|}
\hline Sta. No. & 129 & & Species & Stern: & fusc. & & $\mathrm{SO}$ & $\begin{array}{l}\text { NO. } \\
\text { NO. }\end{array}$ & \\
\hline $\begin{array}{l}\text { Lat \& } \\
\text { Long. }\end{array}$ & Date & $\begin{array}{l}\text { Time } \\
\text { Caught }\end{array}$ & Squid & $\begin{array}{l}\text { Squid } \\
\text { Beaks }\end{array}$ & Fish & $\begin{array}{c}\text { Fish } \\
\text { Remains }\end{array}$ & $\begin{array}{l}\text { Parasitic } \\
\text { Animals }\end{array}$ & Crustacea & $\begin{array}{c}\text { Other } \\
\text { Animals }\end{array}$ \\
\hline $\begin{array}{l}19^{\circ} 40^{\prime} \mathrm{N} \\
163^{\circ} 45^{\prime} \mathrm{W}\end{array}$ & $\begin{array}{l}\text { June } \\
23 \text {, } \\
65\end{array}$ & $\begin{array}{l}\text { Pacific } \\
\text { Ocean }\end{array}$ & & & 2 & + & & & \\
\hline
\end{tabular}

Parasitic Animals:

1ots: 2 fishes

Notes on Squid:

fish remains

Notes on Fish:

Heads aigested.

General Notes:

PACIFIC OCEANOGRAPHIC BIOLOGICAL SURVEY PROGRAM

SMITHSONIAN OCEANOGRAPHIC SORTING CENTER—SMITHSONIAN INSTITUTION

SI-USNM-830

$\lim _{\rightarrow \rightarrow \infty}$

P. Grisham $8 / 3 / 66$

\begin{tabular}{|c|c|c|c|c|c|c|c|c|c|}
\hline Sta. No. & 1300 & & Specie: & Ster & fus & & \multicolumn{2}{|c|}{$\begin{array}{l}\text { SOSC REF. NO. } \\
\text { POBSP. NO. }\end{array}$} & \\
\hline $\begin{array}{l}\text { Lat \& } \\
\text { Long. }\end{array}$ & Date & $\begin{array}{l}\text { Time } \\
\text { Caught }\end{array}$ & Squid & $\begin{array}{l}\text { Squid } \\
\text { Beaks }\end{array}$ & Fish & $\begin{array}{c}\text { Fish } \\
\text { Remains }\end{array}$ & $\begin{array}{l}\text { Parasitic } \\
\text { Animals }\end{array}$ & Crustacea & $\begin{array}{c}\text { Other } \\
\text { Animals }\end{array}$ \\
\hline $\begin{array}{l}19^{\circ} 40^{\prime} \mathrm{N} \\
163^{\circ} 45^{\circ} \mathrm{W}\end{array}$ & $\begin{array}{l}\text { June } \\
23, \\
65\end{array}$ & $\begin{array}{l}\text { Pacific } \\
\text { Ocean }\end{array}$ & & 2 & & + & & & \\
\hline
\end{tabular}

Parasitic Animals:

Notes on Squid:

10ts: 2 squid beaks. fish remains

Notes on Fish:

Fish remains with bones.

General Notes:

PACIFIC OCEANOGRAPHIC BIOLOGICAL SURVEY PROGRAM SMITHSONIAN OCEANOGRAPHIC SORTING CENTER—SMITHSONIAN INSTITUTION

P. Grisham $8 / 3 / 66$ 


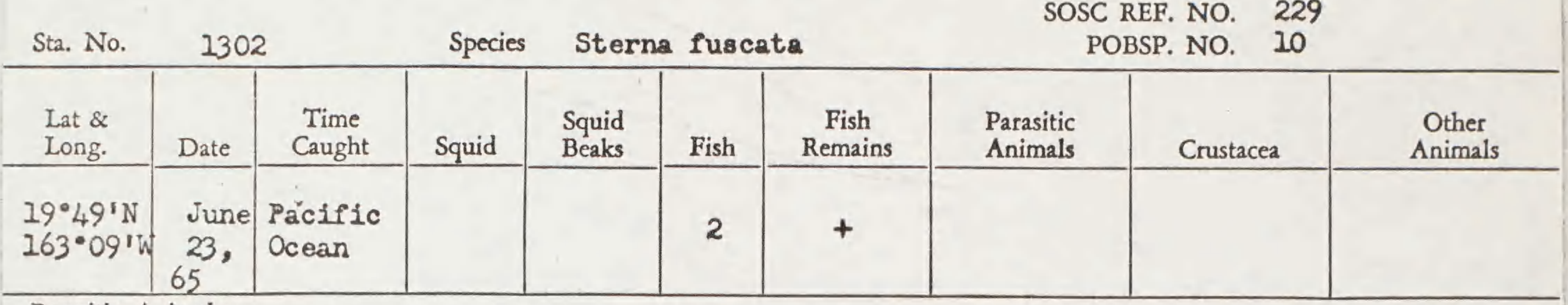

Parasitic Animals:

lots: 2 fish remains

Notes on Squid: Pish

Notes on Fish:

Heads digested.

General Notes:

PACIFIC OCEANOGRAPHIC BIOLOGICAL SURVEY PROGRAM SMITHSONIAN OCEANOGRAPHIC SORTING CENTER—SMITHSONIAN INSTITUTION

P. Grisham 8/8/66

Sta. No.

2306

Species

Puffinus pacificus

POBSP. NO.

SOSC REF. NO. 229

\begin{tabular}{|c|c|c|c|c|c|c|c|c|c|}
\hline $\begin{array}{l}\text { Lat \& } \\
\text { Long. }\end{array}$ & Date & $\begin{array}{l}\text { Time } \\
\text { Caught }\end{array}$ & Squid & $\begin{array}{l}\text { Squid } \\
\text { Beaks }\end{array}$ & Fish & $\begin{array}{c}\text { Fish } \\
\text { Remains }\end{array}$ & $\begin{array}{l}\text { Parasitic } \\
\text { Animals } \\
\end{array}$ & Crustacea & $\begin{array}{c}\text { Other } \\
\text { Animals }\end{array}$ \\
\hline $\begin{array}{l}19^{\circ} 50^{\prime} \mathrm{N} \\
163^{\circ} 04^{\prime} \mathrm{W}\end{array}$ & $\begin{array}{l}\text { June } \\
23, \\
65\end{array}$ & $\begin{array}{l}\text { Pacific } \\
\text { Ocean }\end{array}$ & 3 & 2 & & + & & & \\
\hline
\end{tabular}

Parasitic Animals:

1ots: 5 ereballs (I) squid beaks

Notes on Squid: Heads of two digested. squids fish remains digested remains

Notes on Fish:

General Notes:

PACIFIC OCEANOGRAPHIC BIOLOGICAL SURVEY PROGRAM SMITHSONIAN OCEANOGRAPHIC SORTING CENTER—SMITHSONIAN INSTITUTION 
SOSC REF. NO. 229

Sta. No.

Species

Puffinus pacificus

POBSP. NO. 10

\begin{tabular}{c|c|c|c|c|c|c|c|c|c}
$\begin{array}{c}\text { Lat \& } \\
\text { Long. }\end{array}$ & Date & $\begin{array}{c}\text { Time } \\
\text { Caught }\end{array}$ & Squid & $\begin{array}{c}\text { Squid } \\
\text { Beaks }\end{array}$ & Fish & $\begin{array}{c}\text { Fish } \\
\text { Remains }\end{array}$ & $\begin{array}{c}\text { Parasitic } \\
\text { Animals }\end{array}$ & Crustacea & $\begin{array}{c}\text { Other } \\
\text { Animals }\end{array}$ \\
\hline $\begin{array}{l}19^{\circ} 50^{\prime} \mathrm{N} \\
163^{\circ} 04^{\prime} \mathrm{W}\end{array}$ & $\begin{array}{l}\text { June } \\
23, \\
65\end{array}$ & $\begin{array}{l}\text { Pacific } \\
\text { Ocean }\end{array}$ & 5 & 57 & & + & & & \\
\hline
\end{tabular}

Parasitic Animals:

Notes on Squid:

Heads digested.

10ts: 4 squids squid beaks eyeballs (9) digested remains

Notes on Fish:

General Notes:

Digested remains with a few fish bones.

PACIFIC OCEANOGRAPHIC BIOLOGICAL SURVEY PROGRAM

SMITHSONIAN OCEANOGRAPHIC SORTING CENTER—SMITHSONIAN INSTITUTION

S1-USNM-อ30

$\lim _{\rightarrow \rightarrow 5}$
P. Grisham $8 / 8 / 66$
SMITHSON

7

SOSC REF. NO. 229

Sta. No. $\quad 13 M_{4}$

Species

Fregata minor

POBSP. NO. 10

\begin{tabular}{|c|c|c|c|c|c|c|c|c|c|}
\hline $\begin{array}{l}\text { Lat \& } \\
\text { Long. }\end{array}$ & Date & $\begin{array}{l}\text { Time } \\
\text { Caught }\end{array}$ & Squid & $\begin{array}{l}\text { Squid } \\
\text { Beaks }\end{array}$ & Fish & $\begin{array}{c}\text { Fish } \\
\text { Remains }\end{array}$ & $\begin{array}{l}\text { Parasitic } \\
\text { Animals }\end{array}$ & Crustacea & $\begin{array}{l}\text { Other } \\
\text { Animals }\end{array}$ \\
\hline $\begin{array}{l}20^{\circ} 28^{\prime} \mathrm{N} \\
160^{\circ} 49^{\prime} \mathrm{W}\end{array}$ & $\begin{array}{l}\text { June } \\
24, \\
65\end{array}$ & $\begin{array}{l}\text { Pacific } \\
\text { Ocean }\end{array}$ & & 12 & & + & & & \\
\hline
\end{tabular}

Parasitic Animals:

Notes on Squid:

lots: 2 fish remains squid beaks

Notes on Fish:

Fish remains with bones.

General Notes:

PACIFIC OCEANOGRAPHIC BIOLOGICAL SURVEY PROGRAM

SMITHSONIAN OCEANOGRAPHIC SORTING CENTER—SMITHSONIAN INSTITUTION 


\begin{tabular}{|c|c|c|c|c|c|c|c|c|c|}
\hline Sta. No. & 133] & & Speci & & La 8 & & & $\begin{array}{l}\text { NO. } \\
\text { NO. }\end{array}$ & \\
\hline $\begin{array}{l}\text { Lat \& } \\
\text { Long. }\end{array}$ & Date & $\begin{array}{l}\text { Time } \\
\text { Caught }\end{array}$ & Squid & $\begin{array}{l}\text { Squid } \\
\text { Beaks }\end{array}$ & Fish & $\begin{array}{c}\text { Fish } \\
\text { Remains }\end{array}$ & $\begin{array}{l}\text { Parasitic } \\
\text { Animals }\end{array}$ & Crustacea & $\begin{array}{c}\text { Other } \\
\text { Animals }\end{array}$ \\
\hline $\begin{array}{l}20^{\circ} 48^{\prime} \mathrm{N} \\
159^{\circ} 24^{\prime} \mathrm{W}\end{array}$ & $\begin{array}{l}\text { June } \\
24, \\
65\end{array}$ & $\begin{array}{l}\text { Pacific } \\
\text { Ocean }\end{array}$ & & & 4 & + & & & \\
\hline
\end{tabular}

Parasitic Animals:

Notes on Squid:

lots: 2 fish remains fish

\section{Notes on Fish:}

Heads digested.

General Notes:

PACIFIC OCEANOGRAPHIC BIOLOGICAL SURVEY PROGRAM

SMITHSONIAN OCEANOGRAPHIC SORTING CENTER—SMITHSONIAN INSTITUTION

P. Grisham 8/9/66

S1-USNas-630

$7-68$

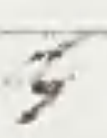

\begin{tabular}{|c|c|c|c|c|c|c|c|c|c|}
\hline Sta. No. & 1345 & & Specic & & inus & elficus & & $\begin{array}{l}\text { NO. } \\
\text { NO. }\end{array}$ & \\
\hline $\begin{array}{l}\text { Lat \& } \\
\text { Long. }\end{array}$ & Date & $\begin{array}{c}\text { Time } \\
\text { Caught }\end{array}$ & Squid & $\begin{array}{l}\text { Squid } \\
\text { Beaks }\end{array}$ & Fish & $\begin{array}{c}\text { Fish } \\
\text { Remains }\end{array}$ & $\begin{array}{l}\text { Parasitic } \\
\text { Animals }\end{array}$ & Crustacea & $\begin{array}{c}\text { Other } \\
\text { Animals }\end{array}$ \\
\hline $\begin{array}{l}16^{\circ} 33^{\prime} \mathrm{N} \\
170^{\circ} 55^{\prime} \mathrm{W}\end{array}$ & $\begin{array}{l}\text { JuIy } \\
10, \\
65\end{array}$ & $\begin{array}{l}\text { Pacific } \\
\text { Ocean }\end{array}$ & 1 & & 4 & + & & & \\
\hline
\end{tabular}

Parasitic Animals:

Notes on Squid:

Head digested.

lots: 3 fish remains

Iish

squid

Notes on Fish:

Fair condition.

General Notes:

PACIFIC OCEANOGRAPHIC BIOLOGICAL SURVEY PROGRAM

SMITHSONIAN OCEANOGRAPHIC SORTING CENTER—SMITHSONIAN INSTITUTION

51-USพM- 30

P. Grisham 8/9/66 
SOSC REF. NO. 229

Sta. No. $1353 \quad$ Species Puffinus pacificus POBSP. NO. 10

\begin{tabular}{|c|c|c|c|c|c|c|c|c|c|}
\hline $\begin{array}{l}\text { Lat \& } \\
\text { Long. }\end{array}$ & Date & $\begin{array}{c}\text { Time } \\
\text { Caught }\end{array}$ & Squid & $\begin{array}{l}\text { Squid } \\
\text { Beaks }\end{array}$ & Fish & $\begin{array}{c}\text { Fish } \\
\text { Remains }\end{array}$ & $\begin{array}{l}\text { Parasitic } \\
\text { Animals }\end{array}$ & Crustacea & $\begin{array}{l}\text { Other } \\
\text { Animals }\end{array}$ \\
\hline $\begin{array}{l}16 \cdot 25^{\prime} \mathrm{N} \\
172 \cdot 31 ' \mathrm{~W}\end{array}$ & $\begin{array}{l}\text { July } \\
10, \\
65\end{array}$ & $\begin{array}{l}\text { Pacific } \\
\text { Ocean }\end{array}$ & 5 & 28 & 1 & - & & & \\
\hline
\end{tabular}

Parasitic Animals:

lots: 6 squids

Notes on Squid: 1. Heads of four digested; one in good condition.

2. Squid remains with tentacles and half of a squid.

Notes on Fish:

1. Fish in poor condition.

squid beaks eyeballs (4) squid remains

fish

digested remains

General Notes:

\begin{tabular}{|c|c|}
\hline & $\begin{array}{l}\text { PACIFIC OCEANOGRAPHIC BIOLOGICAL SURVEY PROGRAM } \\
\text { SMITHSONIAN OCEANOGRAPHIC SORTING CENTER-SMITHSONIAN INSTITUTION }\end{array}$ \\
\hline $\lim _{7 \rightarrow 05}$ & P. Grjsham 8/8/66 \\
\hline
\end{tabular}

\begin{tabular}{|c|c|c|c|c|c|c|c|c|c|}
\hline Sta. No. & \multicolumn{2}{|c|}{1357} & Speci & \multicolumn{3}{|c|}{ Phaethon rubricauda } & \multicolumn{2}{|c|}{$\begin{array}{l}\text { SOSC REF. NO. } 229 \\
\text { POBSP. NO. } 10\end{array}$} & \\
\hline $\begin{array}{l}\text { Lat \& } \\
\text { Long. }\end{array}$ & Date & $\begin{array}{l}\text { Time } \\
\text { Caught }\end{array}$ & Squid & $\begin{array}{l}\text { Squid } \\
\text { Beaks }\end{array}$ & Fish & $\begin{array}{c}\text { Fish } \\
\text { Remains }\end{array}$ & $\begin{array}{l}\text { Parasitic } \\
\text { Animals }\end{array}$ & Crustacea & $\begin{array}{l}\text { Other } \\
\text { Animals }\end{array}$ \\
\hline $\begin{array}{l}14 \cdot 38^{\prime} \mathrm{N} \\
173 \cdot 48 \cdot \mathrm{W}\end{array}$ & $\begin{array}{l}\text { July } \\
\frac{17}{65},\end{array}$ & $\begin{array}{l}\text { Pacific } \\
\text { Ocean }\end{array}$ & & 7 & & + & & & \\
\hline
\end{tabular}

Parasitic Animals:

Notes on Squid:

10ts: 3 ejeballs (1) squid beaks

fish remains

Notes on Fish:

Fish remains with bones.

General Notes:

PACIFIC OCEANOGRAPHIC BIOLOGICAL SURVEY PROGRAM

SMITHSONIAN OCEANOGRAPHIC SORTING CENTER—SMITHSONIAN INSTITUTION

51-บะNม-

P. Grisham 8/23/66 
SOSC REF. NO. 229

Sta. No. 1358

Species Sterna fuscata

POBSP. NO. 10

\begin{tabular}{|c|c|c|c|c|c|c|c|c|c|}
\hline $\begin{array}{l}\text { Lat \& } \\
\text { Long. }\end{array}$ & Date & $\begin{array}{c}\text { Time } \\
\text { Caught }\end{array}$ & Squid & $\begin{array}{l}\text { Squid } \\
\text { Beaks }\end{array}$ & Fish & $\begin{array}{c}\text { Fish } \\
\text { Remains }\end{array}$ & $\begin{array}{l}\text { Parasitic } \\
\text { Animals }\end{array}$ & Crustacea & $\begin{array}{c}\text { Other } \\
\text { Animals }\end{array}$ \\
\hline $\begin{array}{l}16^{\circ} 04^{\prime} \mathrm{N} \\
174^{\circ} 11^{\prime} \mathrm{W}\end{array}$ & $\begin{array}{l}\text { JuIy } \\
\frac{11}{65},\end{array}$ & $\begin{array}{l}\text { Pacific } \\
\text { Ocean }\end{array}$ & 3 & & & + & & & \\
\hline
\end{tabular}

Parasitic Animals:

1ots: 3 squits

Notes on Squid:

Heads of two digested.

squid remains

fish remains

Notes on Fish:

Fish remains with bones.

General Notes:

PACIFIC OCEANOGRAPHIC BIOLOGICAL SURVEY PROGRAM

SMITHSONIAN OCEANOGRAPHIC SORTING CENTER—SMITHSONIAN INSTITUTION

SI-USN-6m-30

$7 \rightarrow 68$

P. Grisham $8 / 15 / 66$

\begin{tabular}{|c|c|c|c|c|c|c|c|c|c|}
\hline Sta. No. & 1359 & & Specie & Sterr & fusc: & & & $\begin{array}{l}\text { NO. } \\
\text { No. }\end{array}$ & \\
\hline $\begin{array}{l}\text { Lat \& } \\
\text { Long. }\end{array}$ & Date & $\begin{array}{l}\text { Time } \\
\text { Caught }\end{array}$ & Squid & $\begin{array}{l}\text { Squid } \\
\text { Beaks }\end{array}$ & Fish & $\begin{array}{c}\text { Fish } \\
\text { Remains }\end{array}$ & $\begin{array}{l}\text { Parasitic } \\
\text { Animals }\end{array}$ & Crustacea & $\begin{array}{l}\text { Other } \\
\text { Animals }\end{array}$ \\
\hline $\begin{array}{l}15^{\circ} 04^{\prime} \mathrm{N} \\
174^{\circ} \mathrm{I} I^{\prime} \mathrm{W}\end{array}$ & $\begin{array}{l}\text { July } \\
211 \\
65\end{array}$ & $\begin{array}{l}\text { Pacific } \\
\text { Ocean }\end{array}$ & 1 & & 1 & + & & & \\
\hline
\end{tabular}

Notes on Squid:

Tentacles attached.

lots: 3 fish

squid

rish remains

Notes on Fish:

Head digested.

General Notes:

PACIFIC OCEANOGRAPHIC BIOLOGICAL SURVEY PROGRAM

SMITHSONIAN OCEANOGRAPHIC SORTING CENTER-SMITHSONIAN INSTITUTION

P. Grisham 8/4/66 
Sta. No. 1360

\begin{tabular}{c|c|l}
\begin{tabular}{c|c} 
Lat \& \\
Long.
\end{tabular} & Date & $\begin{array}{l}\text { Time } \\
\text { Caught }\end{array}$ \\
\cline { 1 - 2 } $\begin{array}{ll}15^{\circ} 04^{\prime} \mathrm{N} \\
174^{\circ} 11^{\prime}\end{array}$ & $\begin{array}{l}\text { JuIJ } \\
\frac{11}{65}\end{array}$ & $\begin{array}{l}\text { Pacific } \\
\text { Ocean }\end{array}$ \\
\hline
\end{tabular}

Parasitic Animals:
SOSC REF. NO. 229

POBSP. NO. 10

Notes on Squid:

lots: 2 fish remains squid beaks

Notes on Fish:

General Notes:

Species Sterna fuscats

\begin{tabular}{|c|c|c|} 
Crustacea & $\begin{array}{c}\text { Other } \\
\text { Animals }\end{array}$ \\
\hline &
\end{tabular}

\section{General Notes:}
PACIFIC OCEANOGRAPHIC BIOLOGICAL SURVEY PROGRAM SMITHSONIAN OCEANOGRAPHIC SORTING CENTER—SMITHSONIAN INSTITUTION
S1-USMm-830
P. Grisham 8/1/66
$\operatorname{lic}_{7-6 s}$

\begin{tabular}{|c|c|c|c|c|c|c|c|c|c|}
\hline Sta. No. & \multicolumn{2}{|c|}{1361} & Specie & \multicolumn{3}{|c|}{ Sterna fuscata } & \multicolumn{2}{|c|}{$\begin{array}{r}\text { SOSC REF. NO. } 22 \\
\text { POBSP. NO. } 10 \\
\end{array}$} & \\
\hline $\begin{array}{l}\text { Lat \& } \\
\text { Long. }\end{array}$ & Date & $\begin{array}{l}\text { Time } \\
\text { Caught }\end{array}$ & Squid & $\begin{array}{l}\text { Squid } \\
\text { Beaks }\end{array}$ & Fish & $\begin{array}{c}\text { Fish } \\
\text { Remains }\end{array}$ & $\begin{array}{l}\text { Parasitic } \\
\text { Animals }\end{array}$ & Crustacea & $\begin{array}{l}\text { Other } \\
\text { Animals }\end{array}$ \\
\hline $\begin{array}{l}15^{\circ} 04^{\prime} \mathrm{N} \\
174^{\circ} 11^{\prime} \mathrm{W}\end{array}$ & $\begin{array}{l}\text { July } \\
11, \\
65\end{array}$ & $\begin{array}{l}\text { Pacific } \\
\text { Ocean }\end{array}$ & 2 & & & $\infty$ & & & \\
\hline
\end{tabular}

Parasitic Animals:

Iots: 2 squids

Notes on Squid:

1. Heads digested.

squid remains

2. Squjid remains with tentacles/6

Notes on Fish:

General Notes:

PACIFIC OCEANOGRAPHIC BIOLOGICAL SURVEY PROGRAM

SMITHSONIAN OCEANOGRAPHIC SORTING CENTER—SMITHSONIAN INSTITUTION 


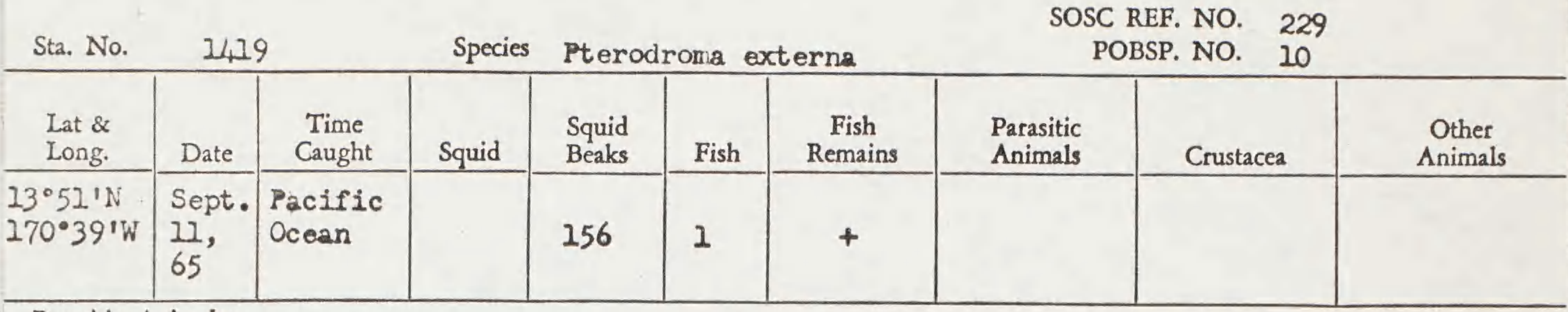

Parasitic Animals:

Notes on Squid:

1. Squid beaks are of various sizes.

1ots: 3 squid beaks

fish

fish remains

Notes on Fish: 1. Fish remains with squid beak fragments.

2. Head of fish digested.

General Notes:

PACIFIC OCEANOGRAPHIC BIOLOGICAL SURVEY PROGRAM

SMITHSONIAN OCEANOGRAPHIC SORTING CENTER_SMITHSONIAN INSTITUTION

Si-USNam-930

$7-68$

Sta. No.

1420

Species Pterodroma externa

\begin{tabular}{|c|c|c|c|c|c|c|}
\hline $\begin{array}{l}\text { Lat \& } \\
\text { Long. }\end{array}$ & Date & $\begin{array}{l}\text { Time } \\
\text { Caught }\end{array}$ & Squid & $\begin{array}{l}\text { Squid } \\
\text { Beaks }\end{array}$ & Fish & $\begin{array}{c}\text { Fish } \\
\text { Remains }\end{array}$ \\
\hline $\begin{array}{l}23^{\circ} 30^{\prime} \mathrm{N} \\
170^{\circ} 48^{\prime} \mathrm{W}\end{array}$ & $\begin{array}{l}\text { Sept. } \\
\frac{17}{65} \text {, }\end{array}$ & $\begin{array}{l}\text { Pacific } \\
\text { Ocean }\end{array}$ & & & & - \\
\hline
\end{tabular}

Parasitic Animals:

Notes on Squid:

Notes on Fish:

General Notes:

Digested remains beyond identification.

PACIFIC OCEANOGRAPHIC BIOLOGICAL SURVEY PROGRAM

SMITHSONIAN OCEANOGRAPHIC SORTING CENTER_-SMITHSONIAN INSTITUTION

S1-USNm-eso

P. Grisham 8/9/66
SOSC REF. NO. 229

POBSP. NO. 10

\begin{tabular}{|c|c|c|}
$\begin{array}{c}\text { Parasitic } \\
\text { Animals }\end{array}$ & Crustacea & $\begin{array}{c}\text { Other } \\
\text { Animals }\end{array}$ \\
\hline & & \\
& & \\
\hline
\end{tabular}

lots: 1 digested remains 
SOSC REF. NO. 229

Sta. No. $\quad u_{421}$ Species Pterodroma externa POBSP. NO. 10

\begin{tabular}{c|c|c|c|c|c|c|c|c|c}
\hline $\begin{array}{c}\text { Lat \& } \\
\text { Long. }\end{array}$ & Date & $\begin{array}{c}\text { Time } \\
\text { Caught }\end{array}$ & Squid & $\begin{array}{c}\text { Squid } \\
\text { Beaks }\end{array}$ & Fish & $\begin{array}{c}\text { Fish } \\
\text { Remains }\end{array}$ & $\begin{array}{c}\text { Parasitic } \\
\text { Animals }\end{array}$ & Crustacea & $\begin{array}{c}\text { Other } \\
\text { Animals }\end{array}$ \\
\hline $\begin{array}{l}10^{\circ} 12^{\prime} \mathrm{N} \\
172^{\circ} \mathrm{I}^{\prime} \mathrm{W}\end{array}$ & $\begin{array}{l}\text { Sept. } \\
12, \\
65\end{array}$ & $\begin{array}{l}\text { Pacific } \\
\text { Ocean }\end{array}$ & 6 & 46 & & - & & & \\
\hline
\end{tabular}

Parasitic Animals:

lots: 4 squids

Notes on Squid: 1. Heads of squids digested.

2. Squid remains with squid beak fragments and squid beaks tentacles. squid remains eyebalis (14)

Notes on Fish:

General Notes:

PACIFIC OCEANOGRAPHIC BIOLOGICAL SURVEY PROGRAM

SMITHSONIAN OCEANOGRAPHIC SORTING CENTER—SMITHSONIAN INSTITUTION

S1-USNM-830

$7-68$
Si-us
P. Grisham $8 / 1 / 66$

-

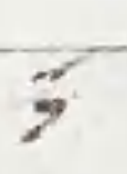

Sta. No.

1422

Species

Sula dactylatra

SOSC REF. NO. 229

\begin{tabular}{|c|c|c|c|c|c|c|c|c|c|}
\hline $\begin{array}{l}\text { Lat \& } \\
\text { Long. }\end{array}$ & Date & $\begin{array}{l}\text { Time } \\
\text { Caught }\end{array}$ & Squid & $\begin{array}{l}\text { Squid } \\
\text { Beaks }\end{array}$ & Fish & $\begin{array}{c}\text { Fish } \\
\text { Remains }\end{array}$ & $\begin{array}{l}\text { Parasitic } \\
\text { Animals }\end{array}$ & Crustacea & $\begin{array}{c}\text { Other } \\
\text { Animals }\end{array}$ \\
\hline $\begin{array}{l}09^{\circ} 10^{\prime} \mathrm{N} \\
172^{\circ} 44^{\prime} \mathrm{W}\end{array}$ & $\begin{array}{l}\text { Sept. } \\
12, \\
65\end{array}$ & $\begin{array}{l}\text { Pacific } \\
\text { Ocean }\end{array}$ & & - & & + & & & \\
\hline
\end{tabular}

Parasitic Animals:

Notes on Squid:

lots: 1 fish remains

Notes on Fish:

Fish remains with half of a fish and bones.

General Notes:

PACIFIC OCEANOGRAPHIC BIOLOGICAL SURVEY PROGRAM

SMITHSONIAN OCEANOGRAPHIC SORTING CENTER—SMITHSONIAN INSTITUTION

S1-USMM-830

$\operatorname{sic}_{x \rightarrow 3}$

P. Grisham 9/14/66 


\begin{tabular}{|c|c|c|c|c|c|c|c|c|c|}
\hline $\begin{array}{l}\text { Lat \& } \\
\text { Long. }\end{array}$ & Date & $\begin{array}{l}\text { Time } \\
\text { Caught }\end{array}$ & Squid & $\begin{array}{l}\text { Squid } \\
\text { Beaks }\end{array}$ & Fish & $\begin{array}{c}\text { Fish } \\
\text { Remains }\end{array}$ & $\begin{array}{l}\text { Parasitic } \\
\text { Animals }\end{array}$ & Crustacea & $\begin{array}{l}\text { Other } \\
\text { Animals }\end{array}$ \\
\hline $\begin{array}{l}09^{\circ} 04^{\prime} \mathrm{N} \\
172^{\circ} 49^{\prime} \mathrm{W}\end{array}$ & $\begin{array}{l}\text { Sept. } \\
12, \\
65\end{array}$ & $\begin{array}{l}\text { Pacific } \\
\text { Ocean }\end{array}$ & & & & + & & & \\
\hline
\end{tabular}

Parasitic Animals:

1ots: 1 fish remains

Notes on Squid:

Notes on Fish:

Fish remains with half of a fish.

General Notes:

PACIFIC OCEANOGRAPHIC BIOLOGICAL SURVEY PROGRAM

SMITHSONIAN OCEANOGRAPHIC SORTING CENTER—SMITHSONIAN INSTITUTION

S1-U3NM-190

P. Grisham 8/23/66

$\operatorname{lic}_{7 \rightarrow-6}$

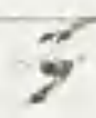

\begin{tabular}{|c|c|c|c|c|c|c|c|c|c|}
\hline \multirow{2}{*}{$\begin{array}{l}\text { Sta. No. } \\
\text { Lat \& } \\
\text { Long. }\end{array}$} & \multicolumn{2}{|c|}{1425} & Species & \multicolumn{2}{|c|}{ Pterodroma } & externa & \multicolumn{2}{|c|}{$\begin{aligned} & \text { SOSC REF. NO. } 22 \\
& \text { POBSP. NO. } 10 \\
&\end{aligned}$} & \multirow[b]{2}{*}{$\begin{array}{c}\text { Other } \\
\text { Animals }\end{array}$} \\
\hline & Date & $\begin{array}{l}\text { Time } \\
\text { Caught }\end{array}$ & Squid & $\begin{array}{l}\text { Squid } \\
\text { Beaks }\end{array}$ & Fish & $\begin{array}{c}\text { Fish } \\
\text { Remains }\end{array}$ & $\begin{array}{l}\text { Parasitic } \\
\text { Animals }\end{array}$ & Crustacea & \\
\hline $\begin{array}{l}6^{\circ} 22^{\prime} \mathrm{N} \\
173^{\circ} 48^{\prime} \mathrm{W}\end{array}$ & $\begin{array}{l}\text { Sept. } \\
13 \\
65\end{array}$ & $\begin{array}{l}\text { Pacific } \\
\text { Ocean }\end{array}$ & & 166 & & - & & & \\
\hline
\end{tabular}

Parasitic Animals:

lats: 3 squid beaks

Notes on Squid:

Squid beaks are of various sizes. eyebalis (4) digested remains

Notes on Fish:

General Notes:

PACIFIC OCEANOGRAPHIC BIOLOGICAL SURVEY PROGRAM

SMITHSONIAN OCEANOGRAPHIC SORTING CENTER—SMITHSONIAN INSTITUTION

$\sin _{7 \rightarrow 05}^{-0.65 m-30}$

P. Grisham 8/16/66 
SOSC REF. NO. 229

Sta. No. $\quad 1426 \quad$ Species Puffinus pacificus

POBSP. NO. 10

\begin{tabular}{c|c|c|c|c|c|c|c|c|c}
\hline $\begin{array}{c}\text { Lat \& } \\
\text { Long. }\end{array}$ & Date & $\begin{array}{c}\text { Time } \\
\text { Caught }\end{array}$ & Squid & $\begin{array}{c}\text { Squid } \\
\text { Beaks }\end{array}$ & Fish & $\begin{array}{c}\text { Fish } \\
\text { Remains }\end{array}$ & $\begin{array}{c}\text { Parasitic } \\
\text { Animals }\end{array}$ & Crustacea & $\begin{array}{c}\text { Other } \\
\text { Animals }\end{array}$ \\
\hline $\begin{array}{l}05^{\circ} 45^{\prime} \mathrm{N} \\
174^{\circ} 04^{\prime} \mathrm{W}\end{array}$ & $\begin{array}{c}\text { Sept. } \\
13 \\
65\end{array}$ & $\begin{array}{c}\text { Pacific } \\
\text { Ocean }\end{array}$ & & 26 & & - & & & \\
\hline
\end{tabular}

Parasitic Animals:

Notes on Squid:

lots: 2 squid beaks digested remains

Notes on Fish:

General Notes:

Digested remains beyond identification.

PACIFIC OCEANOGRAPHIC BIOLOGICAL SURVEY PROGRAM

SMITHSONIAN OCEANOGRAPHIC SORTING CENTER—SMITHSONIAN INSTITUTION

P. Grisham $8 / 4 / 66$

51. USNAB-630

$7 \rightarrow \infty$

\section{F}

SOSC REF. NO. 229

Sta. No. 1427

Species Puffinus pacificus

POBSP. NO. 10

\begin{tabular}{|c|c|c|c|c|c|c|c|c|c|}
\hline $\begin{array}{l}\text { Lat \& } \\
\text { Long. }\end{array}$ & Date & $\begin{array}{c}\text { Time } \\
\text { Caught }\end{array}$ & Squid & $\begin{array}{l}\text { Squid } \\
\text { Beaks }\end{array}$ & Fish & $\begin{array}{c}\text { Fish } \\
\text { Remains }\end{array}$ & $\begin{array}{l}\text { Parasitic } \\
\text { Animals }\end{array}$ & Crustacea & $\begin{array}{l}\text { Other } \\
\text { Animals }\end{array}$ \\
\hline $\begin{array}{l}05^{\circ} 45^{\prime} \mathrm{N} \\
174^{\circ} \mathrm{O} 4^{\prime} \mathrm{W}\end{array}$ & $\begin{array}{l}\text { Sept. } \\
13, \\
65\end{array}$ & $\begin{array}{l}\text { Pacific } \\
\text { Ocean }\end{array}$ & & & & - & & & \\
\hline
\end{tabular}

Parasitic Animals:

lots: I digested remains

Notes on Squid:

Notes on Fish:

General Notes:

Digested remains with pieces of wood.

PACIFIC OCEANOGRAPHIC BIOLOGICAL SURVEY PROGRAM

SMITHSONIAN OCEANOGRAPHIC SORTING CENTER — SMITHSONIAN INSTITUTION

$\operatorname{lic}_{7 \rightarrow 0}$

P. Grisham 7/27/66 
SOSC REF. NO. 229

Sta. No. $\quad 2428 \quad$ Species Puffinus griseus

POBSP. NO. 10

\begin{tabular}{|c|c|c|c|c|c|c|c|c|c|}
\hline $\begin{array}{l}\text { Lat \& } \\
\text { Long. }\end{array}$ & Date & $\begin{array}{l}\text { Time } \\
\text { Caught }\end{array}$ & Squid & $\begin{array}{l}\text { Squid } \\
\text { Beaks }\end{array}$ & Fish & $\begin{array}{c}\text { Fish } \\
\text { Remains }\end{array}$ & $\begin{array}{l}\text { Parasitic } \\
\text { Animals }\end{array}$ & Crustacea & $\begin{array}{c}\text { Other } \\
\text { Animals }\end{array}$ \\
\hline $\begin{array}{l}05^{\circ} 45^{\prime} \mathrm{N} \\
174^{\circ} 04^{\prime} \mathrm{W}\end{array}$ & $\begin{array}{l}\text { Sept. } \\
\frac{13}{65},\end{array}$ & $\begin{array}{l}\text { Pacific } \\
\text { acean }\end{array}$ & & 5 & & - & & & \\
\hline
\end{tabular}

Parasitic Animals:

Notes on Squid:

10ts: 2 squid beaks digested remains

\section{Notes on Fish:}

General Notes:

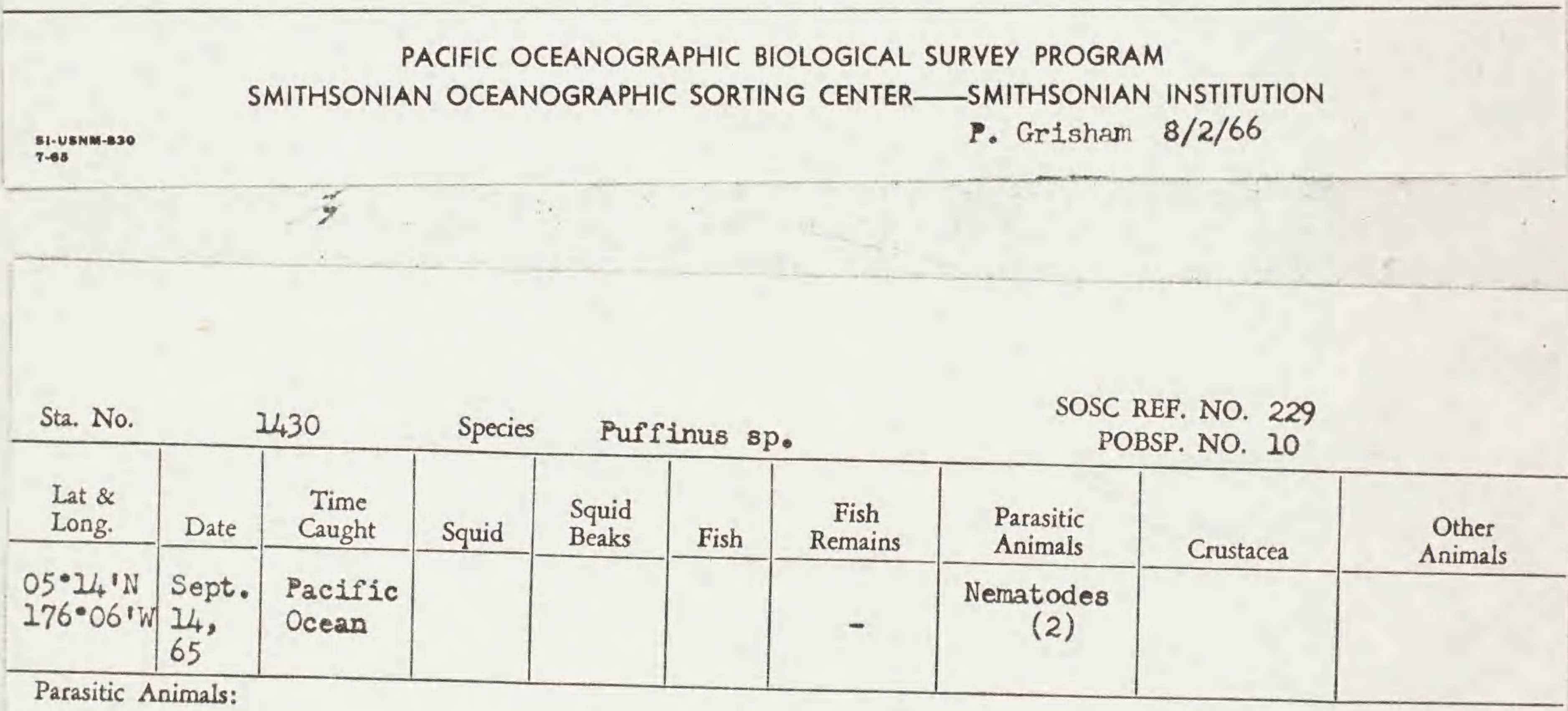

Notes on Squid:

lots: 2 nematodes digested remains

Notes on Fish:

General Notes:

Digested remains beyond identification.

PACIFIC OCEANOGRAPHIC BIOLOGICAL SURVEY PROGRAM

SMITHSONIAN OCEANOGRAPHIC SORTING CENTER—SMITHSONIAN INSTITUTION

P. Grisham 8/3/66 
SOSC REF. NO. 229

\begin{tabular}{|c|c|c|c|c|c|c|c|c|c|}
\hline $\begin{array}{l}\text { Lat \& } \\
\text { Long. }\end{array}$ & Date & $\begin{array}{l}\text { Time } \\
\text { Caught }\end{array}$ & Squid & $\begin{array}{l}\text { Squid } \\
\text { Beaks }\end{array}$ & Fish & $\begin{array}{c}\text { Fish } \\
\text { Remains }\end{array}$ & $\begin{array}{l}\text { Parasitic } \\
\text { Animals }\end{array}$ & Crustacea & $\begin{array}{c}\text { Other } \\
\text { Animals }\end{array}$ \\
\hline $\begin{array}{l}05^{\circ} 14^{\prime} \mathrm{N} \\
176^{\circ} 09^{\prime} \mathrm{W}\end{array}$ & $\begin{array}{c}\text { Sept. } \\
\frac{14}{65},\end{array}$ & $\begin{array}{l}\text { Pacific } \\
\text { Ocean }\end{array}$ & & 5 & & - & & & \\
\hline
\end{tabular}

Parasitic Animals:

lots: 3 eyeballs (1)

Notes on Squid: squid beaks digested remains

\section{Notes on Fish:}

General Notes:

PACIFIC OCEANOGRAPHIC BIOLOGICAL SURVEY PROGRAM SMITHSONIAN OCEANOGRAPHIC SORTING CENTER—SMITHSONIAN INSTITUTION

\begin{tabular}{|c|c|c|c|c|c|c|c|c|c|}
\hline Sta. No. & 1432 & & Speci & & inus & cificus & & $\begin{array}{l}\text { NO. } \\
\text { NO. }\end{array}$ & \\
\hline $\begin{array}{l}\text { Lat \& } \\
\text { Long. }\end{array}$ & Date & $\begin{array}{l}\text { Time } \\
\text { Caught }\end{array}$ & Squid & $\begin{array}{l}\text { Squid } \\
\text { Beaks }\end{array}$ & Fish & $\begin{array}{c}\text { Fish } \\
\text { Remains }\end{array}$ & $\begin{array}{l}\text { Parasitic } \\
\text { Animals }\end{array}$ & Crustacea & $\begin{array}{c}\text { Other } \\
\text { Animals }\end{array}$ \\
\hline $\begin{array}{l}05^{\circ} 15^{\prime} \mathrm{N} \\
176^{\circ} 17^{\prime} \mathrm{W}\end{array}$ & $\begin{array}{l}\text { Sept. } \\
\frac{44}{65},\end{array}$ & $\begin{array}{l}\text { Pacific } \\
\text { Ocean }\end{array}$ & & & & + & & & \\
\hline
\end{tabular}

Parasitic Animals:

Notes on Squid: Iots: 2 squid remains fish remains

Squid remains with half of a squid.

Notes on Fish:

General Notes:

PACIFIC OCEANOGRAPHIC BIOLOGICAL SURVEY PROGRAM SMITHSONIAN OCEANOGRAPHIC SORTING CENTER—SMITHSONIAN INSTITUTION 
SOSC REF. NO. 229

\begin{tabular}{c|l} 
Sta. No. & 1433 \\
\hline \begin{tabular}{c|} 
Lat \& \\
Long.
\end{tabular} & Date \\
\hline $05^{\circ} 16^{\prime} \mathrm{N}$ & Sept. \\
$176^{\circ} 24_{4} \cdot \mathrm{W}$ & $\begin{array}{l}14, \\
65\end{array}$ \\
\hline
\end{tabular}

Parasitic Animals:

\begin{tabular}{|c|c|c|c|c|c|c|c|}
\hline $\begin{array}{c}\text { Time } \\
\text { Caught }\end{array}$ & Squid & $\begin{array}{c}\text { Squid } \\
\text { Beaks }\end{array}$ & Fish & $\begin{array}{c}\text { Fish } \\
\text { Remains }\end{array}$ & $\begin{array}{c}\text { Parasitic } \\
\text { Animals }\end{array}$ & Crustacea & $\begin{array}{c}\text { Other } \\
\text { Animals }\end{array}$ \\
\hline $\begin{array}{l}\text { Pacific } \\
\text { Ocean }\end{array}$ & & & & & & \\
\end{tabular}

lots: 1 digested remains

Notes on Squid:

\section{Notes on Fish:}

General Notes:

Digested remains beyond identification.

PACIFIC OCEANOGRAPHIC BIOLOGICAL SURVEY PROGRAM

SMITHSONIAN OCEANOGRAPHIC SORTING CENTER-SMITHSONIAN INSTITUTION

SI-USNM-830

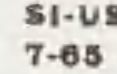

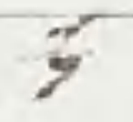

SOSC REF. NO. 229

\begin{tabular}{|c|c|c|c|c|c|c|c|c|c|}
\hline \multirow{2}{*}{$\begin{array}{l}\text { Sta. No. } \\
\text { Lat \& } \\
\text { Long. }\end{array}$} & \multicolumn{2}{|c|}{1434} & Species & \multicolumn{3}{|c|}{ Puffinus tenulrostris } & \multicolumn{2}{|c|}{$\begin{aligned} \text { SOSC REF. NO. } & 22 \\
\text { POBSP. NO. } & 10\end{aligned}$} & \multirow[b]{2}{*}{$\begin{array}{c}\text { Other } \\
\text { Animals }\end{array}$} \\
\hline & Date & $\begin{array}{l}\text { Time } \\
\text { Caught }\end{array}$ & Squid & $\begin{array}{l}\text { Squid } \\
\text { Beaks }\end{array}$ & Fish & $\begin{array}{c}\text { Fish } \\
\text { Remains }\end{array}$ & $\begin{array}{l}\text { Parasitic } \\
\text { Animals }\end{array}$ & Crustacea & \\
\hline $\begin{array}{l}05^{\circ} 17^{\prime} \mathrm{N} \\
176^{\circ} 26^{\prime} \mathrm{W}\end{array}$ & $\begin{array}{l}\text { Sept. } \\
\frac{14}{65}\end{array}$ & $\begin{array}{l}\text { Pacific } \\
\text { Ocean }\end{array}$ & & & & - & & & \\
\hline
\end{tabular}

Parasitic Animals:

Lats: I digested ramains

Notes on Squid:

Notes on Fish:

General Notes:

Digested remains beyond identification.

PACIFIC OCEANOGRAPHIC BIOLOGICAL SURVEY PROGRAM

SMITHSONIAN OCEANOGRAPHIC SORTING CENTER_SMITHSONIAN INSTITUTION

P. Grisham 8/15/66 


\begin{tabular}{|c|c|c|c|c|c|c|c|c|c|}
\hline Sta. No. & $u_{435}$ & & Species & Sterna & fusc & & & $\begin{array}{l}\text { NO. } \\
\text { No. }\end{array}$ & \\
\hline $\begin{array}{l}\text { Lat \& } \\
\text { Long. }\end{array}$ & Date & $\begin{array}{c}\text { Time } \\
\text { Caught }\end{array}$ & Squid & $\begin{array}{l}\text { Squid } \\
\text { Beaks }\end{array}$ & Fish & $\begin{array}{c}\text { Fish } \\
\text { Remains }\end{array}$ & $\begin{array}{l}\text { Parasitic } \\
\text { Animals }\end{array}$ & Crustacea & $\begin{array}{l}\text { Other } \\
\text { Animals }\end{array}$ \\
\hline $\begin{array}{l}02^{\circ} 40^{\prime} \mathrm{N} \\
176^{\circ} 27^{\prime} \mathrm{W}\end{array}$ & $\begin{array}{l}\text { Sept. } \\
15 \text {, } \\
65\end{array}$ & $\begin{array}{l}\text { Pacific } \\
\text { Ocean }\end{array}$ & & & & - & & & \\
\hline
\end{tabular}

Parasitic Animals:

Iots: 0

Notes on Squid:

Notes on Fish:

General Notes:

The stomach was empty.

PACIFIC OCEANOGRAPHIC BIOLOGICAL SURVEY PROGRAM

SMITHSONIAN OCEANOGRAPHIC SORTING CENTER — SMITHSONIAN INSTITUTION

S1-USNM-830

P. Grisham $8 / 1 / 66$

$7-65$

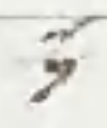

\begin{tabular}{|c|c|c|c|c|c|c|c|c|c|}
\hline $\begin{array}{l}\text { Lat \& } \\
\text { Long. }\end{array}$ & Date & $\begin{array}{l}\text { Time } \\
\text { Caught }\end{array}$ & Squid & $\begin{array}{l}\text { Squid } \\
\text { Beaks }\end{array}$ & Fish & $\begin{array}{c}\text { Fish } \\
\text { Remains }\end{array}$ & $\begin{array}{l}\text { Parasitic } \\
\text { Animals }\end{array}$ & Crustacea & $\begin{array}{l}\text { Other } \\
\text { Animals }\end{array}$ \\
\hline $\begin{array}{l}2^{\circ} 40^{\prime} \mathrm{N} \\
76^{\circ} 27^{\prime} \mathrm{W}\end{array}$ & $\begin{array}{l}\text { Sept. } \\
35 \\
65\end{array}$ & $\begin{array}{l}\text { Pacific } \\
\text { Ocean }\end{array}$ & & & & - & & & \\
\hline
\end{tabular}

Parasitic Animals:

Notes on Squid:

lots: I digested remains

Notes on Fish:

General Notes:

Digested remains beyond identification.

PACIFIC OCEANOGRAPHIC BIOLOGICAL SURVEY PROGRAM

SMITHSONIAN OCEANOGRAPHIC SORTING CENTER—SMITHSONIAN INSTITUTION

S1-USNM-830

7.68

P. Grisham 8/23/66 
SOSC REF. NO. 229

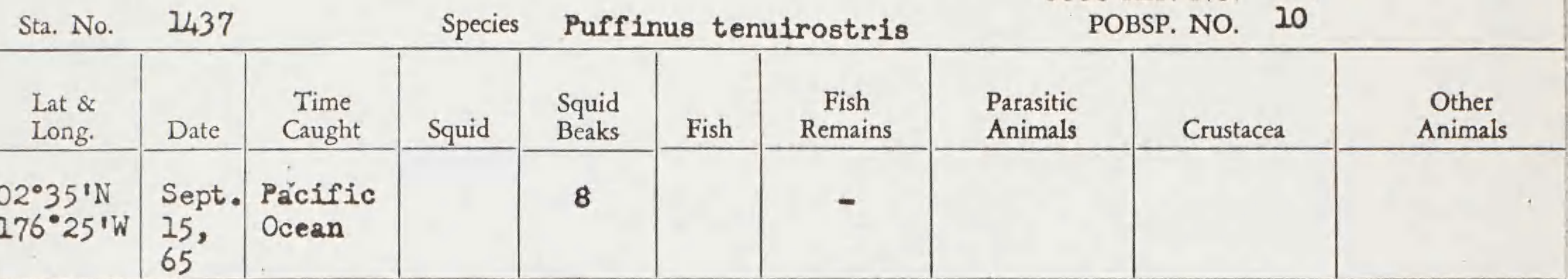

Parasitic Animals:

lots: 2 digested remains

Notes on Squid: squid beaks

Notes on Fish:

General Notes:

PACIFIC OCEANOGRAPHIC BIOLOGICAL SURVEY PROGRAM SMITHSONIAN OCEANOGRAPHIC SORTING CENTER—SMITHSONIAN INSTITUTION

P. Grisham $8 / 9 / 66$

\begin{tabular}{|c|c|c|c|c|c|c|c|c|c|}
\hline Sta. No. & \multicolumn{2}{|c|}{1438} & Specie & \multicolumn{3}{|c|}{ Puffinus pacificus } & \multicolumn{2}{|c|}{ POBSP. NO. 10} & \\
\hline $\begin{array}{l}\text { Lat \& } \\
\text { Long. }\end{array}$ & Date & $\begin{array}{l}\text { Time } \\
\text { Caught }\end{array}$ & Squid & $\begin{array}{l}\text { Squid } \\
\text { Beaks }\end{array}$ & Fish & $\begin{array}{c}\text { Fish } \\
\text { Remains }\end{array}$ & $\begin{array}{l}\text { Parasitic } \\
\text { Animals }\end{array}$ & Crustacea & $\begin{array}{l}\text { Other } \\
\text { Animals }\end{array}$ \\
\hline $\begin{array}{l}2 \cdot 28^{\prime} N \\
76^{\circ} 24^{\prime} \mathrm{W}\end{array}$ & $\begin{array}{l}\text { Sept. } \\
15, \\
65\end{array}$ & $\begin{array}{l}\text { Pacific } \\
\text { Ocean }\end{array}$ & & 8 & & - & & & \\
\hline
\end{tabular}

Parasitic Animals:

Notes on Squid:

1ots: 2 squid beaks digested remains

Notes on Fish:

General Notes:

Digested remains beyond identification.

PACIFIC OCEANOGRAPHIC BIOLOGICAL SURVEY PROGRAM

SMITHSONIAN OCEANOGRAPHIC SORTING CENTER_-SMITHSONIAN INSTITUTION 
SOSC REF. NO. 229

\begin{tabular}{|c|c|c|c|c|c|c|c|c|c|}
\hline $\begin{array}{l}\text { Lat \& } \\
\text { Long. }\end{array}$ & Date & $\begin{array}{c}\text { Time } \\
\text { Caught }\end{array}$ & Squid & $\begin{array}{l}\text { Squid } \\
\text { Beaks }\end{array}$ & Fish & $\begin{array}{c}\text { Fish } \\
\text { Remains }\end{array}$ & $\begin{array}{l}\text { Parasitic } \\
\text { Animals }\end{array}$ & Cfustacea & $\begin{array}{l}\text { Other } \\
\text { Animals }\end{array}$ \\
\hline $\begin{array}{l}02^{\circ} 03^{\prime} \mathrm{N} \\
176^{\circ} 24^{\prime} \mathrm{W}\end{array}$ & $\begin{array}{l}\text { Sept. } \\
15 \text {, } \\
65\end{array}$ & $\begin{array}{l}\text { Pacific } \\
\text { Ocean }\end{array}$ & & & & + & & & \\
\hline
\end{tabular}

Parasitic Animals:

lots: 1 fish remains

Notes on Squid:

Notes on Fish:

Fish remains with bones.

General Notes:

PACIFIC OCEANOGRAPHIC BIOLOGICAL SURVEY PROGRAM

SMITHSONIAN OCEANOGRAPHIC SORTING CENTER—SMITHSONIAN INSTITUTION

P. Grisham 8/31/66

S1.USNM-830

$+5$

\begin{tabular}{|c|c|c|c|c|c|c|c|c|c|}
\hline Sta. No. & 1440 & & Specie & Puf & Ius & ificus & & $\begin{array}{l}\text { NO. } \\
\text { NO. }\end{array}$ & \\
\hline $\begin{array}{l}\text { Lat \& } \\
\text { Long. }\end{array}$ & Date & $\begin{array}{l}\text { Time } \\
\text { Caught }\end{array}$ & Squid & $\begin{array}{l}\text { Squid } \\
\text { Beaks }\end{array}$ & Fish & $\begin{array}{c}\text { Fish } \\
\text { Remains }\end{array}$ & $\begin{array}{l}\text { Parasitic } \\
\text { Animals }\end{array}$ & Crustacea & $\begin{array}{c}\text { Other } \\
\text { Animals }\end{array}$ \\
\hline $\begin{array}{l}01^{\circ} 57^{\prime} \mathrm{N} \\
176^{\circ} 25^{\prime} \mathrm{W}\end{array}$ & $\begin{array}{l}\text { Sept. } \\
15 \\
65\end{array}$ & $\begin{array}{l}\text { Pacific } \\
\text { Ocean }\end{array}$ & & 7 & & - & & & \\
\hline
\end{tabular}

Parasitic Animals:

Notes on Squid:

1ots: 3 squid beaks digested remains debris

Notes on Fish:

General Notes:

Debris with rocks.

PACIFIC OCEANOGRAPHIC BIOLOGICAL SURVEY PROGRAM

SMITHSONIAN OCEANOGRAPHIC SORTING CENTER—SMITHSONIAN INSTITUTION 
SOSC REF. NO.

POBSP. NO.

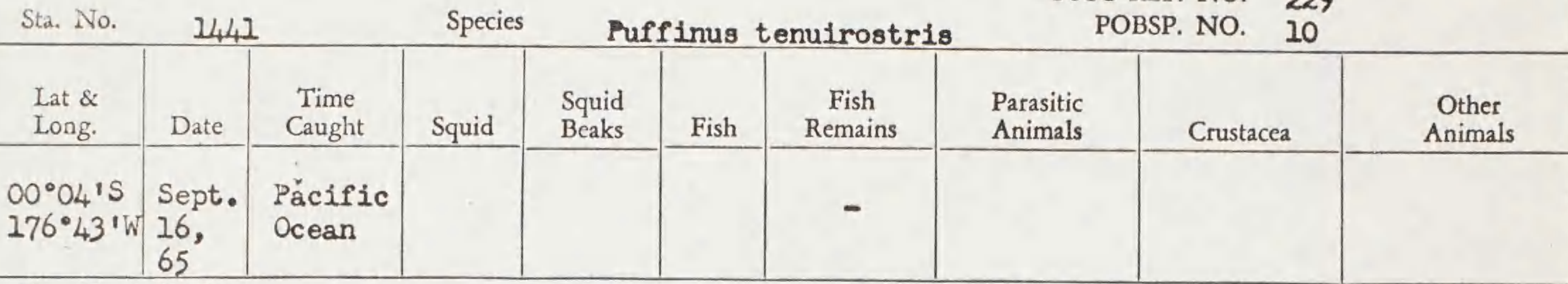

Parasitic Animals:

lots: 1 digested remains

Notes on Squid:

Notes on Fish:

General Notes:

Digested remains beyond identification.

PACIFIC OCEANOGRAPHIC BIOLOGICAL SURVEY PROGRAM

SMITHSONIAN OCEANOGRAPHIC SORTING CENTER—SMITHSONIAN INSTITUTION

SI. USM M.830

7.85

P. Grisham 8/29/66

F

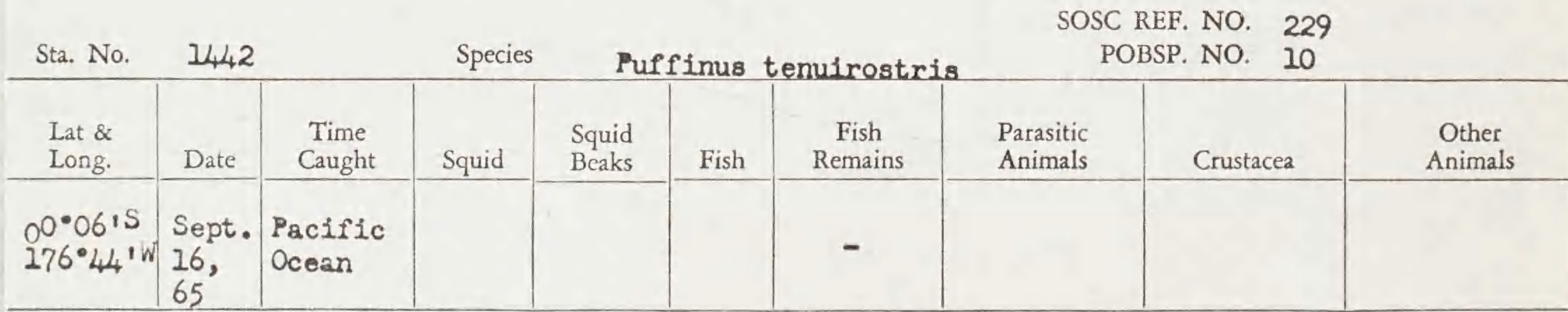

Parasitic Animals:

Notes on Squid:

lats: 1 digested remains

Notes on Fish:

General Notes:

Digested remains beyond identification.

PACIFIC OCEANOGRAPHIC BIOLOGICAL SURVEY PROGRAM

SMITHSONIAN OCEANOGRAPHIC SORTING CENTER—SMITHSONIAN INSTITUTION

$51-45 \mathrm{SMM} \cdot 830$

P. Grisham 9/6/66 
SOSC REF. NO. 229

\begin{tabular}{|c|c|c|c|c|c|c|c|c|c|}
\hline Sta. No. & \multicolumn{2}{|c|}{1443} & Specie & \multicolumn{3}{|c|}{ Pluvialis dominica } & \multicolumn{3}{|c|}{ POBSP. NO. 10} \\
\hline $\begin{array}{l}\text { Lat \& } \\
\text { Long. }\end{array}$ & Date & $\begin{array}{l}\text { Time } \\
\text { Caught }\end{array}$ & Squid & $\begin{array}{l}\text { Squid } \\
\text { Beaks }\end{array}$ & Fish & $\begin{array}{c}\text { Fish } \\
\text { Remains }\end{array}$ & $\begin{array}{l}\text { Parasitic } \\
\text { Animals }\end{array}$ & Crustacea & $\begin{array}{l}\text { Other } \\
\text { Animals }\end{array}$ \\
\hline $\begin{array}{l}00^{\circ} 11^{\prime S} \\
176^{\circ} 49^{\prime} \mathrm{W}\end{array}$ & $\begin{array}{l}\text { Sept } \\
16, \\
65\end{array}$ & $\begin{array}{l}\text { Pacific } \\
\text { Ocean }\end{array}$ & & & & - & & & \\
\hline
\end{tabular}

Parasitic Animals:

lots: 1 digested remains

Notes on Squid:

Notes on Fish:

General Notes:

Digested remains beyond identification.

PACIFIC OCEANOGRAPHIC BIOLOGICAL SURVEY PROGRAM

SMITHSONIAN OCEANOGRAPHIC SORTING CENTER — SMITHSONIAN INSTITUTION

SI-USNM-830

P. Grisham 7/27/66

\begin{tabular}{|c|c|}
\hline $\begin{array}{l}\text { Lat \& } \\
\text { Long. }\end{array}$ & Date \\
\hline $\begin{array}{l}00^{\circ} 23^{\prime S} \\
176^{\circ} 58^{\prime} \mathrm{W}\end{array}$ & $\begin{array}{l}\text { Sept. } \\
16 \text {, } \\
65^{\circ}\end{array}$ \\
\hline
\end{tabular}

Parasitic Animals:

Notes on Squid:

lots: 1 fish remains

Notes on Fish:

Fish remains with bones.

General Notes:

PACIFIC OCEANOGRAPHIC BIOLOGICAL SURVEY PROGRAM

SMITHSONIAN OCEANOGRAPHIC SORTING CENTER—SMITHSONIAN INSTITUTION

$\underset{7-65}{51-U S N M-830}$

P. Grisham $8 / 15 / 66$ 
SOSC REF. NO. 229

Sta. No. $1445 \quad$ Species Anous stolldus

POBSP. NO.

10

\begin{tabular}{|c|c|c|c|c|c|c|c|c|c|}
\hline $\begin{array}{l}\text { Lat \& } \\
\text { Long. }\end{array}$ & Date & $\begin{array}{l}\text { Time } \\
\text { Caught }\end{array}$ & Squid & $\begin{array}{l}\text { Squid } \\
\text { Beaks }\end{array}$ & Fish & $\begin{array}{c}\text { Fish } \\
\text { Remains }\end{array}$ & $\begin{array}{l}\text { Parasitic } \\
\text { Animals }\end{array}$ & Crustacea & $\begin{array}{c}\text { Other } \\
\text { Animals }\end{array}$ \\
\hline $\begin{array}{l}02^{\circ} 19^{\prime} \mathrm{N} \\
177^{\circ} 32^{\prime} \mathrm{W}\end{array}$ & $\begin{array}{l}\text { Sept. } \\
17, \\
65\end{array}$ & $\begin{array}{l}\text { Pácific } \\
\text { Ocean }\end{array}$ & & & & - & $\begin{array}{c}\text { Tapeworms } \\
\text { (1) }\end{array}$ & & \\
\hline
\end{tabular}

Parasitic Animals:

1ots: 2 tapeworm

Notes on Squid:

digested remains

Notes on Fish:

General Notes:

PACIFIC OCEANOGRAPHIC BIOLOGICAL SURVEY PROGRAM SMITHSONIAN OCEANOGRAPHIC SORTING CENTER—SMITHSONIAN INSTITUTION

S1-USNM-830 7.es
Si-us

P. Grisham 8/3/66

SOSC REF. NO. 229

Sta. No.

14,46

Species Anous stolidus

POBSP. NO. 10

\begin{tabular}{|c|c|c|c|c|c|c|c|c|c|}
\hline $\begin{array}{l}\text { Lat \& } \\
\text { Long. }\end{array}$ & Date & $\begin{array}{l}\text { Time } \\
\text { Caught }\end{array}$ & Squid & $\begin{array}{l}\text { Squid } \\
\text { Bcaks }\end{array}$ & Fish & $\begin{array}{c}\text { Fish } \\
\text { Remains }\end{array}$ & $\begin{array}{l}\text { Parasitic } \\
\text { Animals }\end{array}$ & Crustacea & $\begin{array}{c}\text { Other } \\
\text { Animals }\end{array}$ \\
\hline $\begin{array}{l}01 \cdot 19^{1 N} \\
177^{\circ} 32 \mathrm{~W}\end{array}$ & $\begin{array}{l}\text { Sept. } \\
17, \\
65\end{array}$ & $\begin{array}{l}\text { Pacific } \\
\text { Ocean }\end{array}$ & & & & - & & is & \\
\hline
\end{tabular}

Parasitic Animals:

Lots: 0

Notes on Squid:

Notes on Fish:

General Notes:

The stomach was empty.

PACIFIC OCEANOGRAPHIC BIOLOGICAL SURVEY PROGRAM

SMITHSONIAN OCEANOGRAPHIC SORTING CENTER—SMITHSONIAN INSTITUTION

SI-USNM-830

P. Grisham 9/6/66 
SOSC REF. NO. 229

\begin{tabular}{|c|c|c|c|c|c|c|c|c|c|}
\hline Sta. No. & 1448 & & Species & Sul & dact & tra & & NO. & \\
\hline $\begin{array}{l}\text { Lat \& } \\
\text { Long. }\end{array}$ & Date & $\begin{array}{l}\text { Time } \\
\text { Caught }\end{array}$ & Squid & $\begin{array}{l}\text { Squid } \\
\text { Beaks }\end{array}$ & Fish & $\begin{array}{c}\text { Fish } \\
\text { Remains }\end{array}$ & $\begin{array}{l}\text { Parasitic } \\
\text { Animals }\end{array}$ & Crustacea & $\begin{array}{l}\text { Other } \\
\text { Animals }\end{array}$ \\
\hline $\begin{array}{l}01 \bullet 16 \cdot \mathrm{N} \\
176 \div 44^{\prime} \mathrm{W}\end{array}$ & $\begin{array}{l}\text { Sept. } \\
17, \\
65\end{array}$ & $\begin{array}{l}\text { Pacific } \\
\text { Ocean }\end{array}$ & & & 4 & + & & & \\
\hline
\end{tabular}

Parasitic Animals:

lots: 2 fish

Notes on Squid:

Iish remains

Notes on Fish:

Heads of two digested.

General Notes:

No stomach, only stomach contents.

PACIFIC OCEANOGRAPHIC BIOLOGICAL SURVEY PROGRAM

SMITHSONIAN OCEANOGRAPHIC SORTING CENTER—SMITHSONIAN INSTITUTION

P. Grisham 9/13/66

S1-USNM-830
7.65

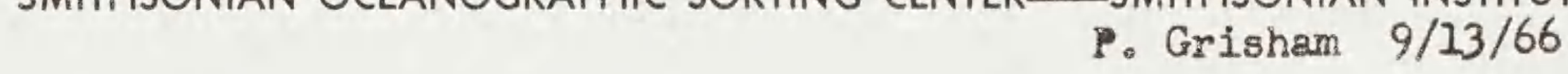

Y

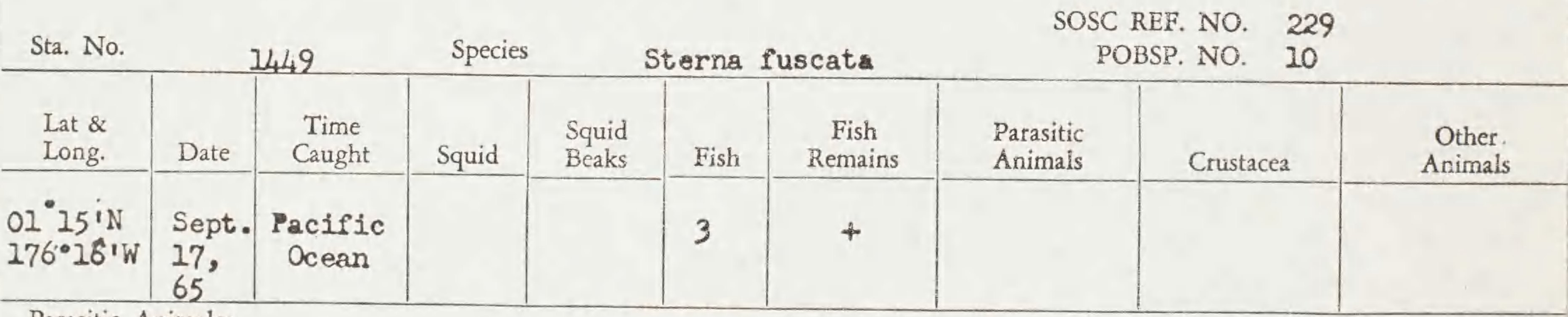

Parasitic Animals:

Notes on Squid:

lots: 2 fish

Sish remains

Notes on Fish:

Heads of two digested.

General Notes:

PACIFIC OCEANOGRAPHIC BIOLOGICAL SURVEY PROGRAM

SMITHSONIAN OCEANOGRAPHIC SORTING CENTER—SMITHSONIAN INSTITUTION

S1.USMM-a30

$7 \cdot 6$

P. Urisham

$8 / 9 / 66$ 


\begin{tabular}{|c|c|c|c|c|c|c|c|c|c|}
\hline Sta. No. & 1450 & & Species & Sula & dacty & tra & $\begin{array}{r}\text { SOSC } \\
\mathrm{F}\end{array}$ & $\begin{array}{l}\text { NO. } \\
\text { NO. }\end{array}$ & \\
\hline $\begin{array}{l}\text { Lat \& } \\
\text { Long. }\end{array}$ & Date & $\begin{array}{c}\text { Time } \\
\text { Caught }\end{array}$ & Squid & $\begin{array}{l}\text { Squid } \\
\text { Beaks }\end{array}$ & Fish & $\begin{array}{c}\text { Fish } \\
\text { Remains }\end{array}$ & $\begin{array}{l}\text { Parasitic } \\
\text { Animals }\end{array}$ & Crustacea & $\begin{array}{l}\text { Other } \\
\text { Animals }\end{array}$ \\
\hline $\begin{array}{l}00^{\circ} 15^{\prime} \mathrm{S} \\
175^{\circ} 56^{\prime} \mathrm{W}\end{array}$ & $\begin{array}{l}\text { Sept } \\
18, \\
65\end{array}$ & $\begin{array}{l}\text { Pacific } \\
\text { Ocean }\end{array}$ & & 21 & & + & $\begin{array}{r}\text { Nematodes } \\
\text { (1) }\end{array}$ & & \\
\hline
\end{tabular}

Notes on Squid:

lots: 4 eyeballs (2)

squid beaks

fish remains

Notes on Fish: nematode

Fish remains with bones.

General Notes:

PACIFIC OCEANOGRAPHIC BIOLOGICAL SURVEY PROGRAM

S1-USNM-630

7.65
SMITHSONIAN OCEANOGRAPHIC SORTING CENTER—SMITHSONIAN INSTITUTION
P. Grisham 8/29/66

\begin{tabular}{|c|c|c|c|c|c|c|c|c|c|}
\hline Stá. No. & $145]$ & & Species & Anc & sto: & & & $\begin{array}{l}\text { NO. } \\
\text { NO. }\end{array}$ & \\
\hline $\begin{array}{l}\text { Lat \& } \\
\text { Long. }\end{array}$ & Date & $\begin{array}{c}\text { Time } \\
\text { Caught }\end{array}$ & Squid & $\begin{array}{l}\text { Squid } \\
\text { Beaks }\end{array}$ & Fish & $\begin{array}{c}\text { Fish } \\
\text { Remains }\end{array}$ & $\begin{array}{l}\text { Parasitic } \\
\text { Animals }\end{array}$ & Crustacea & $\begin{array}{l}\text { Other } \\
\text { Animals }\end{array}$ \\
\hline $\begin{array}{l}00^{\circ} 15^{\prime S} \\
175^{\circ} 56^{\prime} \mathrm{W}\end{array}$ & $\begin{array}{l}\text { Sept. } \\
18, \\
65\end{array}$ & $\begin{array}{l}\text { Pacific } \\
\text { Ocean }\end{array}$ & & & & - & & & \\
\hline
\end{tabular}

Notes on Squid:

Iots: 0

Notes on Fish:

General Notes:

Stomach was empty.

PACIFIC OCEANOGRAPHIC BIOLOGICAL SURVEY PROGRAM

SMITHSONIAN OCEANOGRAPHIC SORTING CENTER — SMITHSONIAN INSTITUTION

P. Grisham $8 / 29 / 66$ 


\begin{tabular}{|c|c|c|c|c|c|c|c|c|c|}
\hline $\begin{array}{l}\text { Lat \& } \\
\text { Long. }\end{array}$ & Date & $\begin{array}{c}\text { Time } \\
\text { Caught }\end{array}$ & Squid & $\begin{array}{l}\text { Squid } \\
\text { Beaks }\end{array}$ & Fish & $\begin{array}{c}\text { Fish } \\
\text { Remains }\end{array}$ & $\begin{array}{l}\text { Parasitic } \\
\text { Animals }\end{array}$ & Crustacea & $\begin{array}{c}\text { Other } \\
\text { Animals }\end{array}$ \\
\hline $\begin{array}{l}00^{\circ} 32^{\prime} \mathrm{S} \\
175^{\circ} 52^{\prime} \mathrm{W}\end{array}$ & $\begin{array}{l}\text { Sept. } \\
18, \\
65\end{array}$ & $\begin{array}{l}\text { Pacific } \\
\text { Ocean }\end{array}$ & & & & + & & & \\
\hline
\end{tabular}

Parasitic Animals:

lots: 1 fish remains

Notes on Squid:

Notes on Fish:

Fish remains with bones.

General Notes:

PACIFIC OCEANOGRAPHIC BIOLOGICAL SURVEY PROGRAM

SMITHSONIAN OCEANOGRAPHIC SORTING CENTER_SMITHSONIAN INSTITUTION

51. USNM-830

P. Grisham 8/16/66

7.05

5

Sta. No.

1453

Species

Arenarla interpres

SOSC REF. NO.

229

POBSP. NO.

10

\begin{tabular}{|c|c|c|c|c|c|c|c|c|c|}
\hline $\begin{array}{l}\text { Lat \& } \\
\text { Long. }\end{array}$ & Date & $\begin{array}{l}\text { Time } \\
\text { Caught }\end{array}$ & Squid & $\begin{array}{l}\text { Squid } \\
\text { Beaks }\end{array}$ & Fish & $\begin{array}{c}\text { Fish } \\
\text { Remains }\end{array}$ & $\begin{array}{l}\text { Parasitic } \\
\text { Animals }\end{array}$ & Crustacea & $\begin{array}{l}\text { Other } \\
\text { Animals }\end{array}$ \\
\hline $\begin{array}{l}01 \cdot 26^{\prime S} \\
176^{\circ} 28^{\prime} \mathrm{W}\end{array}$ & $\begin{array}{l}\text { Sept. } \\
18, \\
65\end{array}$ & $\begin{array}{l}\text { Pacific } \\
\text { Ocean }\end{array}$ & & & & - & & & \\
\hline
\end{tabular}

Parasitic Animals:

Notes on Squid:

lots: 1 digested remains

Notes on Fish:

General Notes:

Digested remains beyond identification.

PACIFIC OCEANOGRAPHIC BIOLOGICAL SURVEY PROGRAM

SMITHSONIAN OCEANOGRAPHIC SORTING CENTER—SMITHSONIAN INSTITUTION

P. Grisham

$8 / 16 / 66$ 


\begin{tabular}{c|l|} 
Sta. No. & \multicolumn{1}{|c|}{1454} \\
\hline Lat \& & \\
Long. & Date \\
\hline $00^{\circ} 15^{\prime} \mathrm{N}$ & Sept. \\
$177^{\circ} 33^{\prime} \mathrm{W}$ & $\begin{array}{l}19, \\
65\end{array}$ \\
\hline
\end{tabular}

Species Sterna fuscata

\begin{tabular}{c|c|c|c|c}
$\begin{array}{c}\text { Time } \\
\text { Caught }\end{array}$ & Squid & $\begin{array}{c}\text { Squid } \\
\text { Beaks }\end{array}$ & Fish & $\begin{array}{c}\text { Fish } \\
\text { Remains }\end{array}$ \\
\cline { 1 - 2 } \\
$\begin{array}{c}\text { Ocean } \\
\text { Cacific }\end{array}$ & & & & + \\
\hline
\end{tabular}

Parasitic Animals:

Notes on Squid:

Notes on Fish:

General Notes:

PACIFIC OCEANOGRAPHIC BIOLOGICAL SURVEY PROGRAM

SMITHSONIAN OCEANOGRAPHIC SORTING CENTER—SMITHSONIAN INSTITUTION

SI-USNM-830

7-85
SOSC REF. NO. 229

POBSP. NO. 10

\begin{tabular}{|c|c|c|}
$\begin{array}{c}\text { Parasitic } \\
\text { Animals }\end{array}$ & Crustacea & $\begin{array}{c}\text { Other } \\
\text { Animals }\end{array}$ \\
\hline & & \\
& & \\
\hline
\end{tabular}

1ots: 1 fish remains

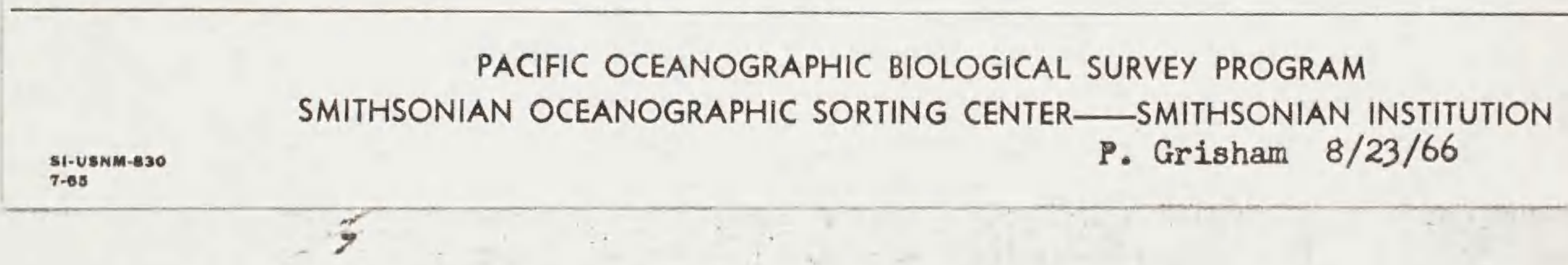

\begin{tabular}{|c|c|c|c|c|c|c|c|c|c|}
\hline Sta. No. & 1455 & & Specie & Ste & $2 f u$ & & SOS & $\begin{array}{l}\text { NO. } \\
\text { NO. }\end{array}$ & \\
\hline $\begin{array}{l}\text { Lat \& } \\
\text { Long. }\end{array}$ & Date & $\begin{array}{c}\text { Time } \\
\text { Caught }\end{array}$ & Squid & $\begin{array}{l}\text { Squid } \\
\text { Beaks }\end{array}$ & Fish & $\begin{array}{c}\text { Fish } \\
\text { Remains }\end{array}$ & $\begin{array}{l}\text { Parasitic } \\
\text { Animals }\end{array}$ & Crustacea & $\begin{array}{c}\text { Other } \\
\text { Animals }\end{array}$ \\
\hline $\begin{array}{l}00^{\circ} 15^{\prime} \mathrm{N} \\
177^{\circ} 33^{\prime} \mathrm{W}\end{array}$ & $\begin{array}{l}\text { Sept. } \\
19, \\
65^{\circ}\end{array}$ & $\begin{array}{l}\text { Pacific } \\
\text { Ocean }\end{array}$ & 2 & 4 & & + & & & \\
\hline
\end{tabular}

Parasitic Animals:

Notes on Squid:

Heads digested.

lots: 4 squids squid beaks digested remains

Notes on Fish: eyeballs (I)

General Notes:

Digested remains with fish bones.

PACIFIC OCEANOGRAPHIC BIOLOGICAL SURVEY PROGRAM SMITHSONIAN OCEANOGRAPHIC SORTING CENTER—SMITHSONIAN INSTIITUTION 
SOSC REF. NO.

229

\begin{tabular}{|c|c|c|c|c|c|c|c|c|c|}
\hline Sta. No. & 1456 & & Specie & Puf & us $p$ & flcus & & No. & \\
\hline $\begin{array}{l}\text { Lat \& } \\
\text { Long. }\end{array}$ & Date & $\begin{array}{c}\text { Time } \\
\text { Caught }\end{array}$ & Squid & $\begin{array}{l}\text { Squid } \\
\text { Beaks }\end{array}$ & Fish & $\begin{array}{c}\text { Fish } \\
\text { Remains }\end{array}$ & $\begin{array}{l}\text { Parasitic } \\
\text { Animals }\end{array}$ & Crustacea & $\begin{array}{c}\text { Other } \\
\text { Animals }\end{array}$ \\
\hline $\begin{array}{l}00^{\circ} 18^{\prime} \mathrm{N} \\
177^{\circ} 29^{\prime} \mathrm{W}\end{array}$ & $\begin{array}{l}\text { Sept. } \\
19, \\
65^{\circ}\end{array}$ & $\begin{array}{l}\text { Pacfic } \\
\text { Ocean }\end{array}$ & 4 & 19 & & - & & & \\
\hline
\end{tabular}

Parasitic Animals:

1ots: 4 squids

Notes on Squid:

Heads digested.

squid remains

squid beaks

ereballs (1)

Notes on Fish:

General Notes:

PACIFIC OCEANOGRAPHIC BIOLOGICAL SURVEY PROGRAM

SMITHSONIAN OCEANOGRAPHIC SORTING CENTER — SMITHSONIAN INSTITUTION

P. Grisham $8 / 8 / 66$

SI-USNM-830

7.65

SOSC REF. NO. 229

POBSP. NO. 10

\begin{tabular}{l|l|l|} 
Sta. No. & \multicolumn{1}{|c|}{1457} \\
\hline $\begin{array}{l}\text { Lat \& } \\
\text { Long. }\end{array}$ & Date & $\begin{array}{l}\text { Time } \\
\text { Caught }\end{array}$ \\
\hline $\begin{array}{ll}00^{\circ} 25^{\prime} \mathrm{N} \\
177^{\circ} 15^{\prime} \mathrm{W}\end{array}$ & $\begin{array}{l}\text { Sept. } \\
19,\end{array}$ & $\begin{array}{l}\text { Pacific } \\
\text { Ocean }\end{array}$ \\
\hline
\end{tabular}

Parasitic Animals:

lots: 4 fish

fish remains

Notes on Squid:

squid beaks

eyeballs (2)

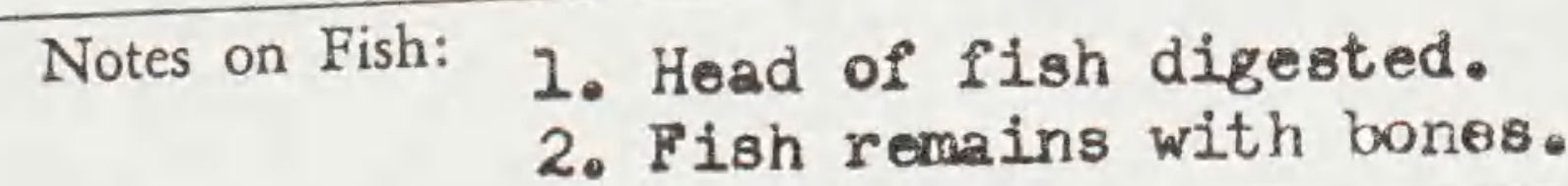

General Notes:

PACIFIC OCEANOGRAPHIC BIOLOGICAL SURVEY PROGRAM SMITHSONIAN OCEANOGRAPHIC SORTING CENTER—SMITHSONIAN INSTITUTION

P. Grisham 7/27/66 
SOSC REF. NO.

\begin{tabular}{c|l} 
Sta. No. & 1458 \\
\hline $\begin{array}{l}\text { Lat \& } \\
\text { Long. }\end{array}$ & Date \\
\hline & \\
$00^{\circ} 40^{\prime} \mathrm{N}$ & Sept. \\
$176^{\circ} 50^{\prime} \mathrm{W}$ & $\begin{array}{l}19, \\
65\end{array}$ \\
\hline
\end{tabular}

Parasitic Animals:

POBSP. NO.

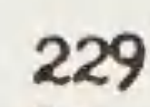

Puffinus paciflcus BSP. NO. 10

Notes on Squid:

lots: 3 squid beaks fish remains eyeba11s (6)

Notes on Fish:

Fish remains with bones.

General Notes:

PACIFIC OCEANOGRAPHIC BIOLOGICAL SURVEY PROGRAM

SMITHSONIAN OCEANOGRAPHIC SORTING CENTER—SMITHSONIAN INSTITUTION

S1-USNM-830

7-65

P. Grisham 7/27/66

SOSC REF. NO. 229

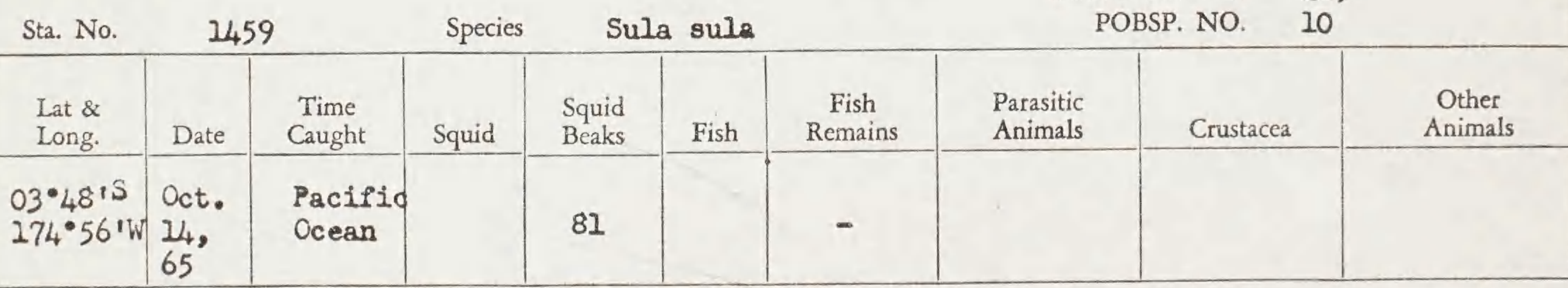

Parasitic Animals:

Notes on Squid:

lots: 3 digested remains squid beaks eyeballs (10)

Notes on Fish:

General Notes:

PACIFIC OCEANOGRAPHIC BIOLOGICAL SURVEY PROGRAM

SMITHSONIAN OCEANOGRAPHIC SORTING CENTER—SMITHSONIAN INSTITUTION

P. Grisham 8/31/66 
SOSC REF. NO. 229

Sta, No.

1460

Arenarla interpres

POBSP. NO.

10

\begin{tabular}{|c|c|c|c|c|c|c|c|c|c|}
\hline $\begin{array}{l}\text { Lat \& } \\
\text { Long. }\end{array}$ & Date & $\begin{array}{c}\text { Time } \\
\text { Caught }\end{array}$ & Squid & $\begin{array}{l}\text { Squid } \\
\text { Beaks }\end{array}$ & Fish & $\begin{array}{c}\text { Fish } \\
\text { Remains }\end{array}$ & $\begin{array}{l}\text { Parasitic } \\
\text { Animals }\end{array}$ & Crustacea & $\begin{array}{l}\text { Other } \\
\text { Animals }\end{array}$ \\
\hline $\begin{array}{l}03 \cdot 48^{\prime} S \\
174 \cdot 59^{\prime} W\end{array}$ & $\begin{array}{l}\text { Oct. } \\
6, \\
65\end{array}$ & $\begin{array}{l}\text { Pacifid } \\
\text { Ocean }\end{array}$ & & & & - & & & \\
\hline
\end{tabular}

Parasitic Animals:

1ots: 0

Notes on Squid:

Notes on Fish:

General Notes:

The stomach was empty.

PACIFIC OCEANOGRAPHIC BIOLOGICAL SURVEY PROGRAM

SMITHSONIAN OCEANOGRAPHIC SORTING CENTER—SMITHSONIAN INSTITUTION

S1-USNM-830

P. Grisham 8/15/66

$7-65$

\%

SOSC REF. NO.

POBSP. NO. 10

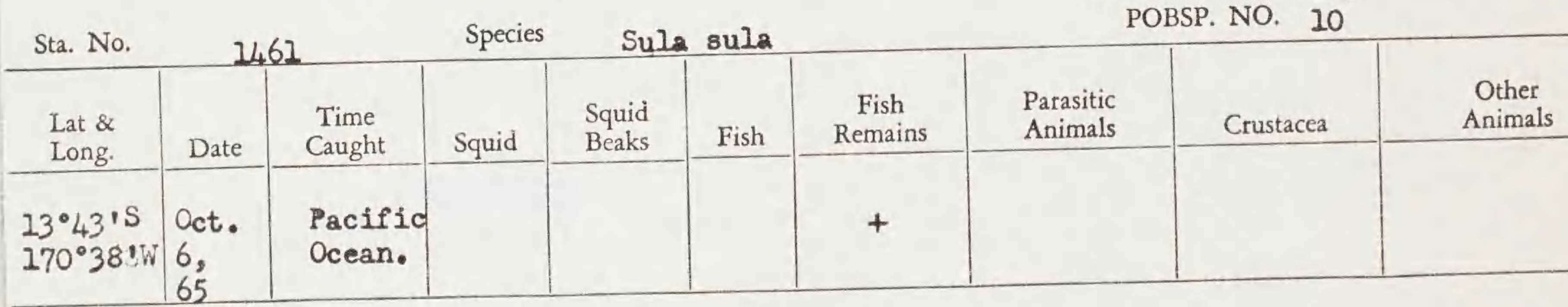

Parasitic Animals:

lots: 1 sish remains

Notes on Squid:

Notes on Fish:

Fish remains with bones.

General Notes:

PACIFIC OCEANOGRAPHIC BIOLOGICAL SURVEY PROGRAM SMITHSONIAN OCEANOGRAPHIC SORTING CENTER—SMITHSONIAN INSTITUTION

P. Grisham 9/6/66 
SOSC REF. NO.

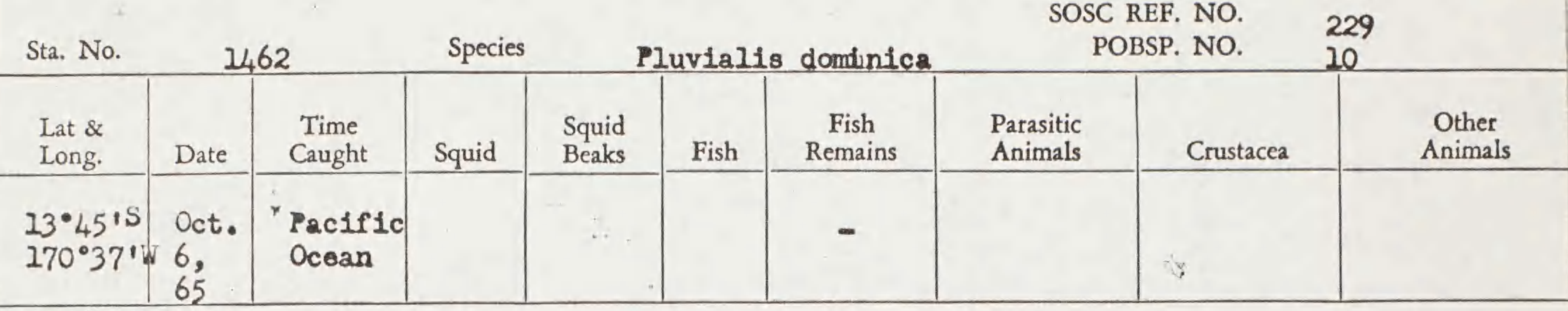

Parasitic Animals:

10ts: 1 digested remalins

Notes on Squid:

Notes on Fish:

General Notes:

PACIFIC OCEANOGRAPHIC BIOLOGICAL SURVEY PROGRAM

SMITHSONIAN OCEANOGRAPHIC SORTING CENTER—SMITHSONIAN INSTITUTION

S1-USNM-830

P. Grisham 8/5/66

$7-65$

SOSC REF. NO. 229

Sta. No.

Species

Sterna fuscata

POBSP. NO.

10

\begin{tabular}{|c|c|c|c|c|c|c|c|c|c|}
\hline $\begin{array}{l}\text { Lat \& } \\
\text { Long. }\end{array}$ & Date & $\begin{array}{c}\text { Time } \\
\text { Caught }\end{array}$ & Squid & $\begin{array}{l}\text { Squid } \\
\text { Beaks }\end{array}$ & Fish & $\begin{array}{c}\text { Fish } \\
\text { Remains }\end{array}$ & $\begin{array}{l}\text { Parasitic } \\
\text { Animals }\end{array}$ & Crustacea & $\begin{array}{c}\text { Other } \\
\text { Animals }\end{array}$ \\
\hline $\begin{array}{l}06^{\circ} 03^{\prime N} \\
173^{\circ} 37^{\prime N}\end{array}$ & $\begin{array}{l}\text { Oct. } \\
13 ! \\
65\end{array}$ & $\begin{array}{l}\text { Pacific } \\
\text { Ocean }\end{array}$ & & & & - & & & \\
\hline
\end{tabular}

Parasitic Animals:

lots: 1 digested remains

Notes on Squid:

Notes on Fish:

General Notes:

Digested remains beyond identification.

PACIFIC OCEANOGRAPHIC BIOLOGICAL SURVEY PROGRAM

SMITHSONIAN OCEANOGRAPHIC SORTING CENTER—SMITHSONIAN INSTITUTION 


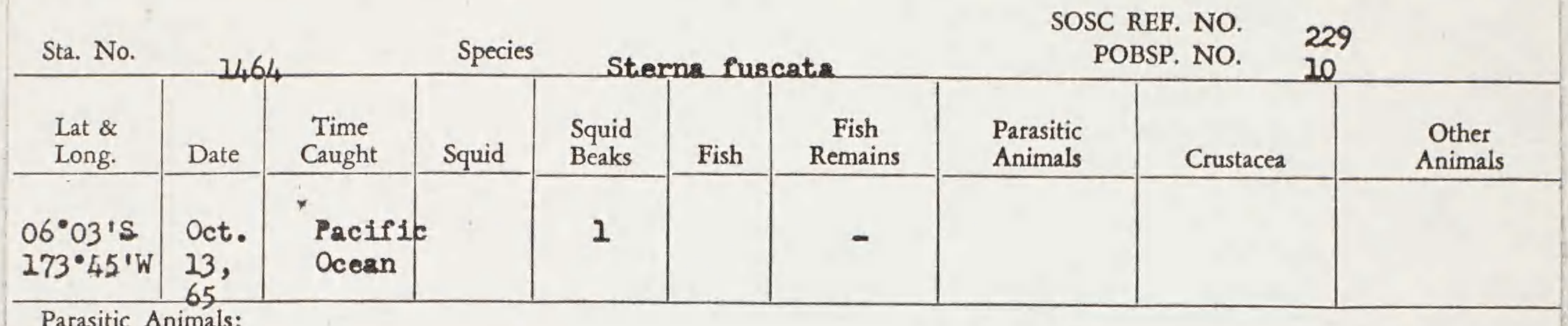

Parasitic Animals:

Notes on Squid:

10ts: 2 digested remains squid beaks

Notes on Fish:

General Notes:

Digested remains beyond identification.

PACIFIC OCEANOGRAPHIC BIOLOGICAL SURVEY PROGRAM

SMITHSONIAN OCEANOGRAPHIC SORTING CENTER—SMITHSONIAN INSTITUTION

S1-UsNm-830

7-65

P. Grisham 8/23/66

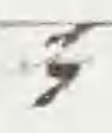

SOSC REF. NO.

POBSP. NO.

229

Sta. No.

1465

Species

Sterna fuscata

BSP. NO. 10

\begin{tabular}{|c|c|c|c|c|c|c|c|c|c|}
\hline $\begin{array}{l}\text { Lat \& } \\
\text { Long. }\end{array}$ & Date & $\begin{array}{l}\text { Time } \\
\text { Caught }\end{array}$ & Squid & $\begin{array}{l}\text { Squid } \\
\text { Beaks }\end{array}$ & Fish & $\begin{array}{c}\text { Fish } \\
\text { Remains }\end{array}$ & $\begin{array}{l}\text { Parasitic } \\
\text { Animals }\end{array}$ & Crustacea & $\begin{array}{c}\text { Other } \\
\text { Animals }\end{array}$ \\
\hline $\begin{array}{l}06^{\circ} 03^{\prime S} \\
173^{\circ} 45^{\prime} \mathrm{W}\end{array}$ & $\begin{array}{l}\text { Oct. } \\
13 \\
65\end{array}$ & $\begin{array}{l}\text { Pacifiq } \\
\text { Dcean }\end{array}$ & 3 & & & + & & & \\
\hline
\end{tabular}

Parasitic Animals:

lots: 3 squids

Notes on Squid: 1. Squid remains with half of a squid.

2. Squids in good condition.

Notes on Fish:

General Notes:

PACIFIC OCEANOGRAPHIC BIOLOGICAL SURVEY PROGRAM

SMITHSONIAN OCEANOGRAPHIC SORTING CENTER—SMITHSONIAN INSTITUTION 
SOSC REF. NO.

Sta. No. 1466

Sterna fuscata POBSP. NO.

10

\begin{tabular}{c|c|c|c|c|c|c|c|c|c}
\hline $\begin{array}{c}\text { Lat \& } \\
\text { Long. }\end{array}$ & Date & $\begin{array}{c}\text { Time } \\
\text { Caught }\end{array}$ & Squid & $\begin{array}{c}\text { Squid } \\
\text { Beaks }\end{array}$ & Fish & $\begin{array}{c}\text { Fish } \\
\text { Remains }\end{array}$ & $\begin{array}{c}\text { Parasitic } \\
\text { Animals }\end{array}$ & $\begin{array}{c}\text { Other } \\
\text { Animals }\end{array}$ \\
\hline $06^{\circ} 03^{\prime} \mathrm{S}$ & $\begin{array}{l}\text { Oct. } \\
173^{\circ} 45^{\prime} \mathrm{W}\end{array}$ & $\begin{array}{l}13, \\
65\end{array}$ & $\begin{array}{l}\text { Páciflc } \\
\text { Ocean }\end{array}$ & 4 & & - & & \\
\hline
\end{tabular}

Parasitic Animals:

lots: 2 squids

Notes on Squid: 1. Head of one digested.

digested remains

\section{Tentacles of two detached.}

Notes on Fish:

General Notes:

PACIFIC OCEANOGRAPHIC BIOLOGICAL SURVEY PROGRAM SMITHSONIAN OCEANOGRAPHIC SORTING CENTER—SMITHSONIAN INSTITUTION

P. Grisham 7/27/66

Sta. No. 1467

Species

Lat \&

Long.

$06^{\circ} 03^{\prime S}$

$173^{\circ} 45^{\prime} \mathrm{W}$

Parasitic Animals:

Notes on Squid:

Good condition.

Notes on Fish:

Heads digested.

General Notes:
Lots: 4 squid beaks fish remains fish squid
SOSC REF. NO. 229 POBSP. NO. 10 
SOSC REF. NO. 229

\begin{tabular}{|c|c|c|c|c|c|c|c|c|c|}
\hline Sta. No. & \multicolumn{2}{|c|}{1468} & Species & \multicolumn{2}{|c|}{ Heteroscelus } & incanum & \multicolumn{2}{|c|}{ POBSP. NO. 10} & \\
\hline $\begin{array}{l}\text { Lat \& } \\
\text { Long. }\end{array}$ & Date & $\begin{array}{l}\text { Time } \\
\text { Caught }\end{array}$ & Squid & $\begin{array}{l}\text { Squid } \\
\text { Beaks }\end{array}$ & Fish & $\begin{array}{c}\text { Fish } \\
\text { Remains }\end{array}$ & $\begin{array}{l}\text { Parasitic } \\
\text { Animals }\end{array}$ & Crustacea & $\begin{array}{l}\text { Other } \\
\text { Animals }\end{array}$ \\
\hline $\begin{array}{l}03^{\circ} 56^{\circ} \mathrm{S} \\
173^{\circ} 48^{\circ} \mathrm{W}\end{array}$ & $\begin{array}{l}\text { Oct. } \\
13, \\
65\end{array}$ & $\begin{array}{l}\text { Pacific } \\
\text { Ocean }\end{array}$ & & & & - & & & \\
\hline
\end{tabular}

Parasitic Animals:

Iots: 0

Notes on Squid:

Notes on Fish:

General Notes:

The'stomach was empty.

PACIFIC OCEANOGRAPHIC BIOLOGICAL SURVEY PROGRAM

SMITHSONIAN OCEANOGRAPHIC SORTING CENTER SMITHSONIAN JNSTITUTION

S1.USMM-830

$7 \rightarrow 05$

$$
3
$$

SOSC REF. NO. 229

\begin{tabular}{|c|c|c|c|c|c|c|c|c|c|}
\hline Sta. No. & 1469 & & Specie & & 8 st & dus & & NO. & \\
\hline $\begin{array}{l}\text { Lat \& } \\
\text { Long. }\end{array}$ & Date & $\begin{array}{l}\text { Time } \\
\text { Caught }\end{array}$ & Squid & $\begin{array}{l}\text { Squid } \\
\text { Beaks }\end{array}$ & Fish & $\begin{array}{c}\text { Fish } \\
\text { Remains }\end{array}$ & $\begin{array}{l}\text { Parasitic } \\
\text { Animals }\end{array}$ & Crustacea & $\begin{array}{l}\text { Other } \\
\text { Animals }\end{array}$ \\
\hline $\begin{array}{l}00^{\circ} 13^{\prime} \text { s } \\
176^{\circ} 09^{\prime} \mathrm{W}\end{array}$ & $\begin{array}{l}\text { Oct. } \\
25 \text {, } \\
65\end{array}$ & $\begin{array}{l}\text { Pacific } \\
\text { Ocean }\end{array}$ & & & & - & & & \\
\hline
\end{tabular}

Parasitic Animals:

lots: 1 digested remains

Notes on Squid:

Notes on Fish:

General Notes:

Digested remains beyond identification.

PACIFIC OCEANOGRAPHIC BIOLOGICAL SURVEY PROGRAM

SMITHSONIAN OCEANOGRAPHIC SORTING CENTER—SMITHSONIAN INSTITUTION 


\begin{tabular}{|c|c|c|c|c|c|c|c|c|c|}
\hline Sta. No. & 147 & & Speci & & finus & tenulros & & $\begin{array}{l}\text { NO. } 22 \\
\text { NO. } 10\end{array}$ & \\
\hline $\begin{array}{l}\text { Lat \& } \\
\text { Long. }\end{array}$ & Date & $\begin{array}{l}\text { Time } \\
\text { Caught }\end{array}$ & Squid & $\begin{array}{l}\text { Squid } \\
\text { Beaks }\end{array}$ & Fish & $\begin{array}{c}\text { Fish } \\
\text { Remains }\end{array}$ & $\begin{array}{l}\text { Parasitic } \\
\text { Animals }\end{array}$ & Crustacea & $\begin{array}{c}\text { Other } \\
\text { Animals }\end{array}$ \\
\hline $\begin{array}{l}00^{\circ} 10^{1 N} \\
176^{\circ} 26^{\prime} \mathrm{W}\end{array}$ & $\begin{array}{l}\text { Oct. } \\
15 \\
65\end{array}$ & $\begin{array}{l}\text { Pacific } \\
\text { Ocean }\end{array}$ & & & & t & & & \\
\hline
\end{tabular}

Parasitic Animals:

Notes on Squid:

lots: 1 digested remains

Notes on Fish:

General Notes:

Digested remains beyond identification.

PACIFIC OCEANOGRAPHIC BIOLOGICAL SURVEY PROGRAM

SMITHSONIAN OCEANOGRAPHIC SORTING CENTER—SMITHSONIAN INSTITUTION
$\operatorname{Ti}_{T-65}-\mathrm{USNM}-830$
P. Grisham 8/5/66

\section{5}

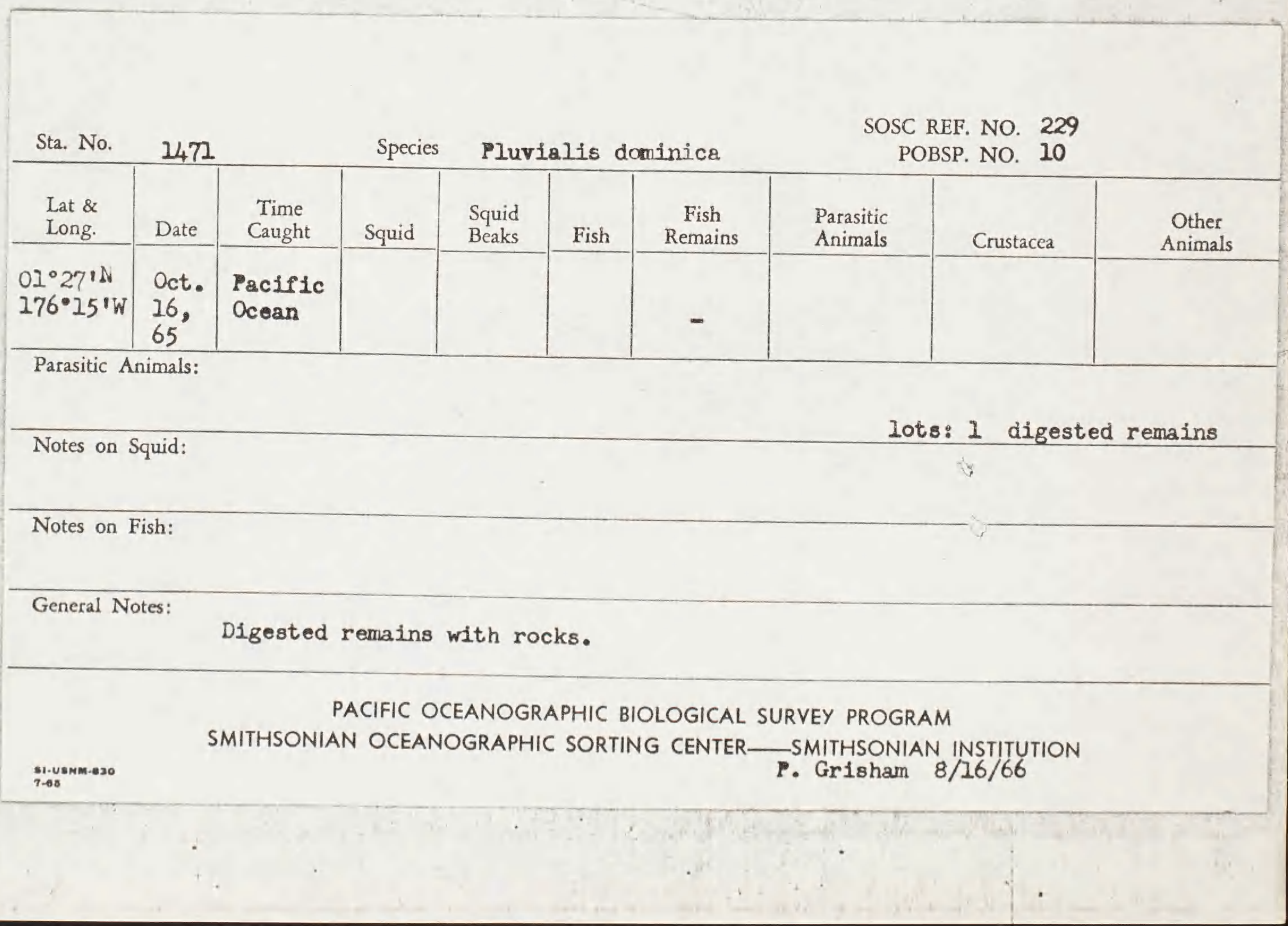




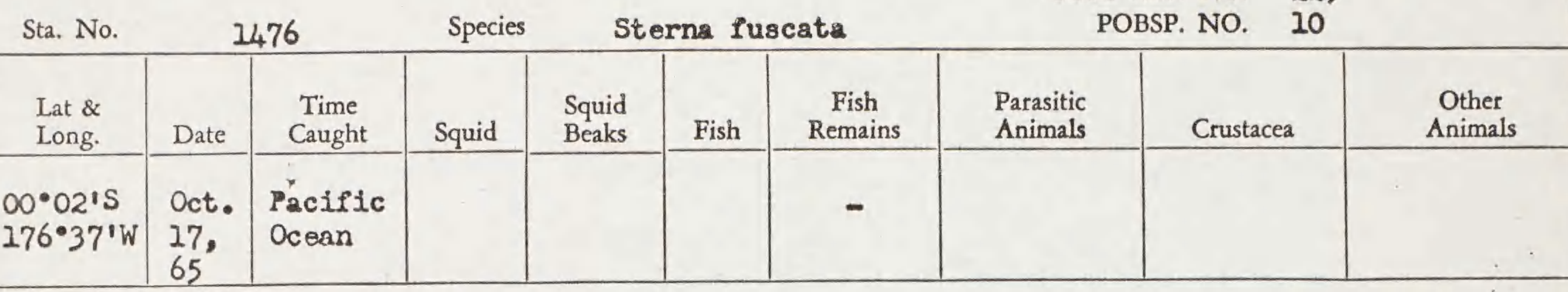

Parasitic Animals:

lots: 1 digested remains

Notes on Squid:

Notes on Fish:

General Notes:

Digested remains beyond identification.

PACIFIC OCEANOGRAPHIC BIOLOGICAL SURVEY PROGRAM

SMITHSONIAN OCEANOGRAPHIC SORTING CENTER—SMITHSONIAN INSTITUTION

S1-USNM-830

P. Grisham $8 / 15 / 66$

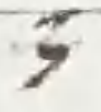

\begin{tabular}{|c|c|c|c|c|c|c|c|c|c|}
\hline $\begin{array}{l}\text { Lat \& } \\
\text { Long. }\end{array}$ & Date & $\begin{array}{l}\text { Time } \\
\text { Caught }\end{array}$ & Squid & $\begin{array}{l}\text { Squid } \\
\text { Beaks }\end{array}$ & Fish & $\begin{array}{c}\text { Fish } \\
\text { Remains }\end{array}$ & $\begin{array}{l}\text { Parasitic } \\
\text { Animals }\end{array}$ & Crustacea & $\begin{array}{c}\text { Other } \\
\text { Animals }\end{array}$ \\
\hline $\begin{array}{l}00^{\circ} 06^{\prime} \mathrm{N} \\
176^{\circ} 50^{\prime} \mathrm{W}\end{array}$ & $\begin{array}{l}\text { Oct. } \\
17, \\
65\end{array}$ & $\begin{array}{l}\text { Pacific } \\
\text { Ocean }\end{array}$ & & & & & & & \\
\hline
\end{tabular}

Parasitic Animals:

Notes on Squid:

Notes on Fish:

General Notes:

The specimen was not received.

PACIFIC OCEANOGRAPHIC BIOLOGICAL SURVEY PROGRAM

SMITHSONIAN OCEANOGRAPHIC SORTING CENTER_SMITHSONIAN INSTITUTION

P. Grisham 9/19/66 


\begin{tabular}{|c|c|c|c|c|c|c|c|c|c|}
\hline Sta. No. & 1482 & & Species & Pte. & roma & inexpectat & & $\begin{array}{l}\text { No. } \\
\text { No. }\end{array}$ & \\
\hline $\begin{array}{l}\text { Lat \& } \\
\text { Long. }\end{array}$ & Date & $\begin{array}{c}\text { Time } \\
\text { Caught }\end{array}$ & Squid & $\begin{array}{l}\text { Squid } \\
\text { Beaks }\end{array}$ & Fish & $\begin{array}{c}\text { Fish } \\
\text { Remains }\end{array}$ & $\begin{array}{l}\text { Parasitic } \\
\text { Animals }\end{array}$ & Crustacea & $\begin{array}{l}\text { Other } \\
\text { Animals }\end{array}$ \\
\hline $\begin{array}{l}00^{\circ} 20^{\prime} \mathrm{N} \\
176^{\circ} 52^{\prime} \mathrm{W}\end{array}$ & $\begin{array}{l}\text { Oct. } \\
17, \\
65\end{array}$ & $\begin{array}{l}\text { Pacific } \\
\text { Ocean }\end{array}$ & & 20 & & - & & & \\
\hline
\end{tabular}

Parasitic Animals:

lots: 2 squid beaks

Notes on Squid:

digested remains

\section{Notes on Fish:}

\section{General Notes:}

PACIFIC OCEANOGRAPHIC BIOLOGICAL SURVEY PROGRAM

\$1-USNM-930

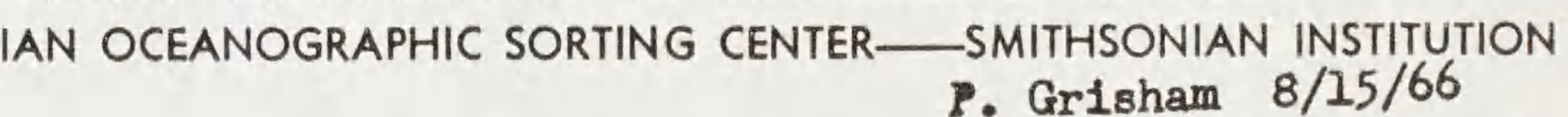

\begin{tabular}{|c|c|c|c|c|c|c|c|c|c|}
\hline \multirow{2}{*}{$\begin{array}{l}\text { Sta. No. } \\
\text { Lat \& } \\
\text { Long. }\end{array}$} & 1485 & \multicolumn{2}{|r|}{ Species } & \multicolumn{2}{|c|}{ Oceanodroma } & castro & \multicolumn{2}{|c|}{$\begin{array}{l}\text { SOSC REF. NO. } \\
\text { POBSP. NO. }\end{array}$} & $\begin{array}{l}229 \\
10\end{array}$ \\
\hline & Date & $\begin{array}{l}\text { Time } \\
\text { Caught }\end{array}$ & Squid & $\begin{array}{l}\text { Squid } \\
\text { Beaks }\end{array}$ & Fish & $\begin{array}{c}\text { Fish } \\
\text { Remains }\end{array}$ & $\begin{array}{l}\text { Parasitic } \\
\text { Animals }\end{array}$ & Crustacea & $\begin{array}{l}\text { Other } \\
\text { Animals }\end{array}$ \\
\hline $\begin{array}{l}02^{\circ} 00^{\prime} \mathrm{N} \\
177^{\circ} 30^{\prime} \mathrm{W}\end{array}$ & $\begin{array}{l}\text { Oct. } \\
18 \text {, } \\
65\end{array}$ & $\begin{array}{l}\text { Pacific } \\
\text { Ocean }\end{array}$ & & & & + & & & \\
\hline
\end{tabular}

Parasitic Animals:

Notes on Squid:

lots: 2 fish remains

4

Notes on Fish:

Fish remains with bones.

General Notes:

PACIFIC OCEANOGRAPHIC BIOLOGICAL SURVEY PROGRAM SMITHSONIAN OCEANOGRAPHIC SORTING CENTER-SMITHSONIAN INSTITUTION 


\begin{tabular}{|c|c|c|c|c|c|c|c|c|c|}
\hline $\begin{array}{l}\text { Lat \& } \\
\text { Long. }\end{array}$ & Date & $\begin{array}{l}\text { Time } \\
\text { Caught }\end{array}$ & Squid & $\begin{array}{l}\text { Squid } \\
\text { Beaks }\end{array}$ & Fish & $\begin{array}{c}\text { Fish } \\
\text { Remains }\end{array}$ & $\begin{array}{l}\text { Parasitic } \\
\text { Animals }\end{array}$ & Crustacea & $\begin{array}{l}\text { Other } \\
\text { Animals }\end{array}$ \\
\hline $\begin{array}{l}01^{\circ} 39^{\prime} \mathrm{N} \\
178^{\circ} 07^{\prime} \mathrm{W}\end{array}$ & $\begin{array}{l}\text { Oct. } \\
18, \\
65\end{array}$ & $\begin{array}{l}\text { Pacific } \\
\text { Ocean }\end{array}$ & & & & + & & & \\
\hline
\end{tabular}

Parasitic Animals:

lots: 1 fish remains

Notes on Squid:

Notes on Fish:

General Notes:

PACIFIC OCEANOGRAPHIC BIOLOGICAL SURVEY PROGRAM

SMITHSONIAN OCEANOGRAPHIC SORTING CENTER—SMITHSONIAN INSTITUTION

P. Grisham $8 / 16 / 66$

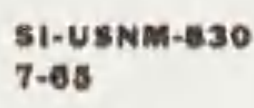

\begin{tabular}{|c|c|c|c|c|c|c|c|c|c|}
\hline Sta. No. & \multicolumn{2}{|c|}{1488} & Speci & \multicolumn{2}{|c|}{ Pluvialis } & dominica & \multicolumn{2}{|c|}{$\begin{array}{l}\text { SOSC REF. NO. } 22 \\
\text { POBSP. NO. } 10\end{array}$} & \\
\hline $\begin{array}{l}\text { Lat \& } \\
\text { Long. }\end{array}$ & Date & $\begin{array}{c}\text { Time } \\
\text { Caught }\end{array}$ & Squid & $\begin{array}{l}\text { Squid } \\
\text { Beaks }\end{array}$ & Fish & $\begin{array}{c}\text { Fish } \\
\text { Remains }\end{array}$ & $\begin{array}{l}\text { Parasitic } \\
\text { Animals }\end{array}$ & Crustacea & $\begin{array}{c}\text { Other } \\
\text { Animals }\end{array}$ \\
\hline $\begin{array}{l}00^{\circ} 15^{\prime} \mathrm{N} \\
177^{\circ} 38^{\prime} \mathrm{W}\end{array}$ & $\begin{array}{l}\text { Oct. } \\
19, \\
65\end{array}$ & $\begin{array}{l}\text { Pacific } \\
\text { Ocean }\end{array}$ & & & & & & & \\
\hline
\end{tabular}

Parasitic Animals:

Notes on Squid:

Notes on Fish:

General Notes:

The specimen was not received.

PACIFIC OCEANOGRAPHIC BIOLOGICAL SURVEY PROGRAM

SMITHSONIAN OCEANOGRAPHIC SORTING CENTER_-SMITHSONIAN INSTITUTION

F. Grisham 9/19/66 
SOSC REF. NO. 229

\begin{tabular}{|c|c|c|c|c|c|c|c|c|c|}
\hline $\begin{array}{l}\text { Lat \& } \\
\text { Long. }\end{array}$ & Date & $\begin{array}{l}\text { Time } \\
\text { Caught }\end{array}$ & Squid & $\begin{array}{l}\text { Squid } \\
\text { Beaks }\end{array}$ & Fish & $\begin{array}{c}\text { Fish } \\
\text { Remains }\end{array}$ & $\begin{array}{l}\text { Parasitic } \\
\text { Animals }\end{array}$ & Crustacea & $\begin{array}{l}\text { Other } \\
\text { Animals }\end{array}$ \\
\hline $\begin{array}{l}00^{\circ} 20^{\prime} \mathrm{N} \\
177^{\circ} 28^{\prime} \mathrm{W}\end{array}$ & $\begin{array}{l}\text { Oct. } \\
19, \\
65\end{array}$ & $\begin{array}{l}\text { Pacific } \\
\text { Ocean }\end{array}$ & & 1 & & - & 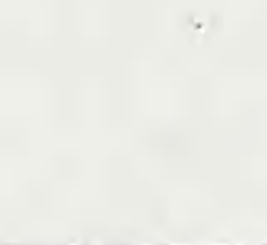 & & \\
\hline
\end{tabular}

Parasitic Animals:

Iots: 2 squid beaks

Notes on Squid:

Notes on Fish:

General Notes:

PACIFIC OCEANOGRAPHIC BIOLOGICAL SURVEY PROGRAM

SMITHSONIAN OCEANOGRAPHIC SORTING CENTER—SMITHSONIAN INSTITUTION

S1-USNM-830

7-65

P. Grisham 8/5/66

\begin{tabular}{|c|c|c|c|c|c|c|c|c|c|}
\hline Sta. No. & 1490 & & Speci & Oce & tes & anicus & & $\begin{array}{l}\text { No. } \\
\text { No. } 1\end{array}$ & \\
\hline $\begin{array}{l}\text { Lat \& } \\
\text { Long. }\end{array}$ & Date & $\begin{array}{l}\text { Time } \\
\text { Caught }\end{array}$ & Squid & $\begin{array}{l}\text { Squid } \\
\text { Beaks }\end{array}$ & Fish & $\begin{array}{c}\text { Fish } \\
\text { Remains }\end{array}$ & $\begin{array}{l}\text { Parasitic } \\
\text { Animals }\end{array}$ & Crustacea & $\begin{array}{l}\text { Other } \\
\text { Animals }\end{array}$ \\
\hline $\begin{array}{l}00^{\circ} 20^{\prime} \mathrm{N} \\
177^{\circ} 28^{\prime} \mathrm{W}\end{array}$ & $\begin{array}{l}\text { Oct. } \\
19, \\
65\end{array}$ & $\begin{array}{l}\text { Pacific } \\
\text { Ocean }\end{array}$ & & & & + & & & \\
\hline
\end{tabular}

Parasitic Animals:

Notes on Squid:

lots: 1 fish remains

Notes on Fish:

General Notes:

PACIFIC OCEANOGRAPHIC BIOLOGICAL SURVEY PROGRAM

SMITHSONIAN OCEANOGRAPHIC SORTING CENTER—SMITHSONIAN INSTITUTION

S1-USNM-830

$7-65$

P. Grisham $8 / 23 / 66$ 


\begin{tabular}{|c|c|c|c|c|c|c|c|c|c|}
\hline Sta. No. & 1491 & & Species & Ocea & es 0 & nicus & SOS & $\begin{array}{l}\text { No. } \\
\text { No. }\end{array}$ & \\
\hline $\begin{array}{l}\text { Lat \& } \\
\text { Long. }\end{array}$ & Date & $\begin{array}{l}\text { Time } \\
\text { Caught }\end{array}$ & Squid & $\begin{array}{l}\text { Squid } \\
\text { Beaks }\end{array}$ & Fish & $\begin{array}{c}\text { Fish } \\
\text { Remains }\end{array}$ & $\begin{array}{l}\text { Parasitic } \\
\text { Animals }\end{array}$ & Crustacea & $\begin{array}{l}\text { Other } \\
\text { Animals }\end{array}$ \\
\hline $\begin{array}{l}00^{\circ} 20^{\prime} \mathrm{N} \\
177^{\circ} 28^{\prime} \mathrm{W}\end{array}$ & $\begin{array}{l}\text { Oct. } \\
19 \text {, } \\
65\end{array}$ & $\begin{array}{l}\text { Pácific } \\
\text { Ocean }\end{array}$ & & & & - & & & \\
\hline
\end{tabular}

lots: 1 digested remains

Notes on Squid:

Notes on Fish:

General Notes:

Digested remains beyond identification.

PACIFIC OCEANOGRAPHIC BIOLOGICAL SURVEY PROGRAM

SMITHSONIAN OCEANOGRAPHIC SORTING CENTER—SMITHSONIAN INSTITUTION

S1-USNM-830

7.65

P. Grisham 8/23/66

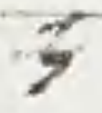

SOSC REF. NO. 229

\begin{tabular}{|c|c|c|c|c|c|c|c|c|c|}
\hline $\begin{array}{l}\text { Lat \& } \\
\text { Long. }\end{array}$ & Date & $\begin{array}{l}\text { Time } \\
\text { Caught }\end{array}$ & Squid & $\begin{array}{l}\text { Squid } \\
\text { Beaks }\end{array}$ & Fish & $\begin{array}{c}\text { Fish } \\
\text { Remains }\end{array}$ & $\begin{array}{l}\text { Parasitic } \\
\text { Animals }\end{array}$ & Crustacea & $\begin{array}{l}\text { Other } \\
\text { Animals }\end{array}$ \\
\hline $\begin{array}{l}00^{\circ} 26^{\prime} \mathrm{s} \\
177^{\circ} 38^{\prime} \mathrm{W}\end{array}$ & $\begin{array}{l}\text { Oct. } \\
19, \\
65\end{array}$ & $\begin{array}{l}\text { Pacific } \\
\text { Ocean }\end{array}$ & & & & - & & & \\
\hline
\end{tabular}

Parasitic Animals:

Notes on Squid:

lots: 1 digested remains

Notes on Fish:

General Notes:

Digested remains beyond identification.

PACIFIC OCEANOGRAPHIC BIOLOGICAL SURVEY PROGRAM

SMITHSONIAN OCEANOGRAPHIC SORTING CENTER—SMITHSONIAN INSTITUTION 
SOSC REF. NO.

229

\begin{tabular}{|c|c|c|}
\hline $\begin{array}{l}\text { Lat \& } \\
\text { Long. }\end{array}$ & Date & $\begin{array}{c}\text { Time } \\
\text { Caught }\end{array}$ \\
\hline $\begin{array}{l}00^{\bullet} 26^{\prime} \mathrm{s} \\
177^{\bullet} 37^{\prime} \mathrm{w}\end{array}$ & $\begin{array}{l}\text { Oct. } \\
20, \\
65\end{array}$ & $\begin{array}{l}\text { Pacific } \\
\text { Ocean }\end{array}$ \\
\hline
\end{tabular}

Species

Pterodroma inexpectata POBSP. NO. 10

Parasitic Animals:

Notes on Squid:

Lots: 2 squid beaks digested remains

\section{Notes on Fish:}

General Notes:

PACIFIC OCEANOGRAPHIC BIOLOGICAL SURVEY PROGRAM SMITHSONIAN OCEANOGRAPHIC SORTING CENTER_SMITHSONIAN INSTITUTION

S1-USNM-830

\section{P. Grisham 8/1/66}

\section{y}

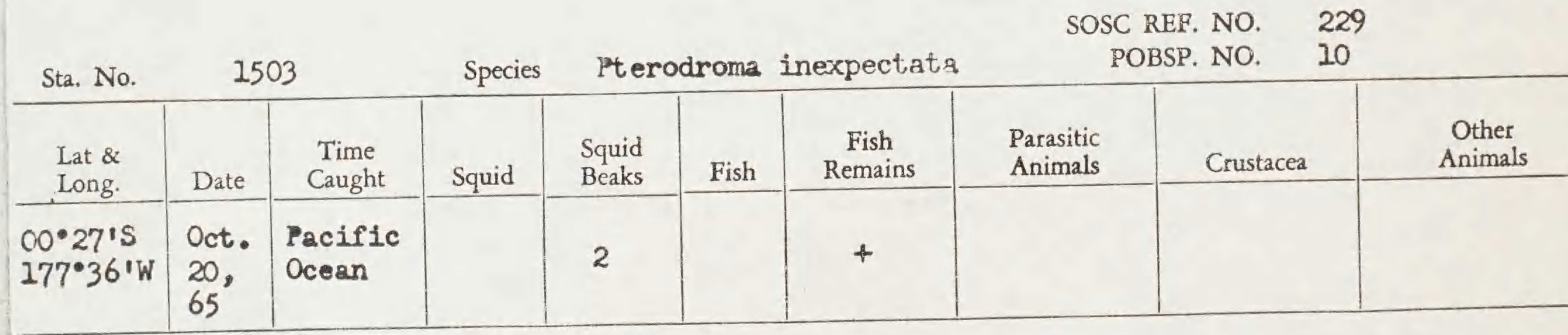

Parasitic Animals:

lots: 2 squid beaks

Notes on Squid: fish remains

Notes on Fish:

General Notes:

PACIFIC OCEANOGRAPHIC BIOLOGICAL SURVEY PROGRAM SMITHSONIAN OCEANOGRAPHIC SORTING CENTER—SMITHSONIAN INSTITUTION 
SOSC REF. NO. 229

Sta. No. Species Sterna fuscata

POBSP. NO. 10

\begin{tabular}{|c|c|c|c|c|c|c|c|c|c|}
\hline $\begin{array}{l}\text { Lat \& } \\
\text { Long. }\end{array}$ & Date & $\begin{array}{l}\text { Time } \\
\text { Caught }\end{array}$ & Squid & $\begin{array}{l}\text { Squid } \\
\text { Beaks }\end{array}$ & Fish & $\begin{array}{c}\text { Fish } \\
\text { Remains }\end{array}$ & $\begin{array}{l}\text { Parasitic } \\
\text { Animals }\end{array}$ & Crustacea & $\begin{array}{c}\text { Other } \\
\text { Animals }\end{array}$ \\
\hline $\begin{array}{l}00^{\circ} 27^{\prime S} \\
177^{\bullet} 36^{\prime} \mathrm{W}\end{array}$ & $\begin{array}{l}\text { Oct. } \\
20, \\
65\end{array}$ & $\begin{array}{l}\text { Pacific } \\
\text { Ocean }\end{array}$ & & & & - & & & \\
\hline
\end{tabular}

Parasitic Animals:

lots: 1 digested remains

Notes on Squid:

Notes on Fish:

General Notes:

Digested remains beyond identification.

PACIFIC OCEANOGRAPHIC BIOLOGICAL SURVEY PROGRAM

SMITHSONIAN OCEANOGRAPHIC SORTING CENTER — SMITHSONIAN INSTITUTION

P. Grisham 7/27/66

7-68

\section{\%}

SOSC RER. NO. 229

\begin{tabular}{|c|c|c|c|c|c|c|c|c|c|}
\hline Sta. No. & 1515 & & Specie: & Ocea & 80 & nfcus & & 180 & \\
\hline $\begin{array}{l}\text { Lat \& } \\
\text { Long. }\end{array}$ & Date & $\begin{array}{c}\text { Time } \\
\text { Caught }\end{array}$ & Squid & $\begin{array}{l}\text { Squid } \\
\text { Beaks }\end{array}$ & Fish & $\begin{array}{c}\text { Fish } \\
\text { Remains }\end{array}$ & $\begin{array}{l}\text { Parasitic } \\
\text { Animals }\end{array}$ & Crustacea & $\begin{array}{c}\text { Other } \\
\text { Animals }\end{array}$ \\
\hline $\begin{array}{l}00^{\circ} 27^{1 S} \\
177^{\circ} 36^{\prime} \mathrm{W}\end{array}$ & $\begin{array}{l}\text { Oct. } \\
20, \\
65\end{array}$ & $\begin{array}{l}\text { Pacific } \\
\text { Ocean }\end{array}$ & & & & + & & $\because$ & \\
\hline
\end{tabular}

Parasitic Animals:

lots: 1 fish remains

Notes on Squid:

Notes on Fish:

General Notes:

PACIFIC OCEANOGRAPHIC BIOLOGICAL SURVEY PROGRAM SMITHSONIAN OCEANOGRAPHIC SORTING CENTER—SMITHSONIAN INSTITUTION 
SOSC REF. NO. 229

\begin{tabular}{|c|c|c|c|c|c|c|c|c|c|}
\hline Sta. No. & & & Speci & Ste & fu: & & & NO. 1 & \\
\hline $\begin{array}{l}\text { Lat \& } \\
\text { Long. }\end{array}$ & Date & $\begin{array}{l}\text { Time } \\
\text { Caught }\end{array}$ & Squid & $\begin{array}{l}\text { Squid } \\
\text { Beaks }\end{array}$ & Fish & $\begin{array}{c}\text { Fish } \\
\text { Remains }\end{array}$ & $\begin{array}{l}\text { Parasitic } \\
\text { Animals }\end{array}$ & Crustacea & $\begin{array}{c}\text { Other } \\
\text { Animals }\end{array}$ \\
\hline $\begin{array}{l}0 \cdot 34^{\prime} \mathrm{N} \\
76^{\circ} 20^{\prime} \mathrm{W}\end{array}$ & $\begin{array}{l}\text { Oct. } \\
22, \\
65\end{array}$ & $\begin{array}{l}\text { Pacific } \\
\text { Ocean }\end{array}$ & & 1 & & - & & & \\
\hline
\end{tabular}

Parasitic Animals:

Notes on Squid:

lots: 2 squid beaks digested remains

Notes on Fish:

General Notes:

Digested remains beyond identification.

PACIFIC OCEANOGRAPHIC BIOLOGICAL SURVEY PROGRAM

SMITHSONIAN OCEANOGRAPHIC SORTING CENTER_SMITHSONIAN INSTITUTION

\$1-USNM -830
$7-65$
2. Grisham $8 / 1 / 66$

.

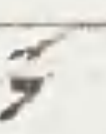

Sta. No.

1522

Species

Sterna fuscata

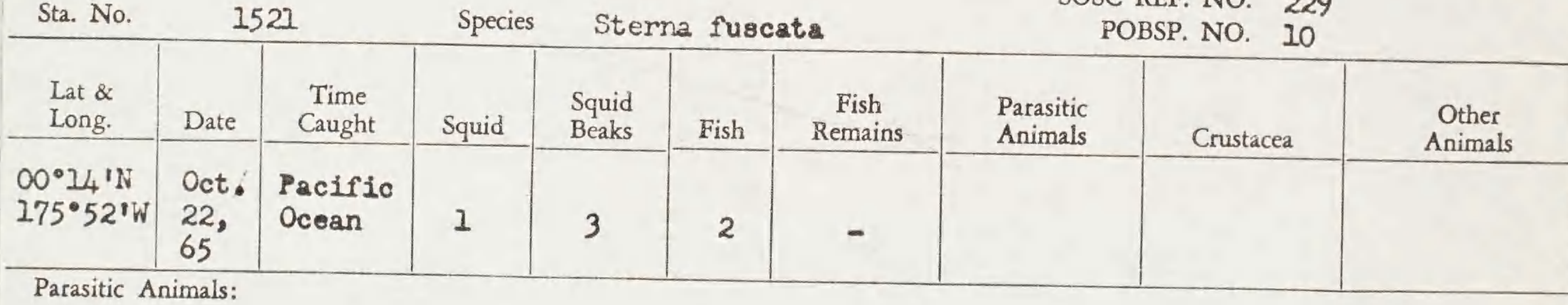

Notes on Squid:

Head digested.

Notes on Fish:

\section{Poor condition.}

Lots: 4 squid

digested remains

fish

squid beaks

General Notes:

PACIFIC OCEANOGRAPHIC BIOLOGICAL SURVEY PROGRAM

SMITHSONIAN OCEANOGRAPHIC SORTING CENTER—SMITHSONIAN INSTITUTION

P. Grisham $8 / 8 / 66$ 
SOSC REF. NO. 229

Sta. No.

Species

Sula sula

POBSP. NO. 10

\begin{tabular}{|c|c|c|c|c|c|c|c|c|c|}
\hline $\begin{array}{l}\text { Lat \& } \\
\text { Long. }\end{array}$ & Date & $\begin{array}{l}\text { Time } \\
\text { Caught }\end{array}$ & Squid & $\begin{array}{l}\text { Squid } \\
\text { Beaks }\end{array}$ & Fish & $\begin{array}{c}\text { Fish } \\
\text { Remains }\end{array}$ & $\begin{array}{l}\text { Parasitic } \\
\text { Animals }\end{array}$ & Crustacea & $\begin{array}{c}\text { Other } \\
\text { Animals }\end{array}$ \\
\hline $\begin{array}{l}02^{\circ} 22^{\prime S} \\
120^{\circ} 43^{\prime} \mathrm{W}\end{array}$ & $\begin{array}{l}\text { Oct. } \\
24, \\
65\end{array}$ & $\begin{array}{l}\text { Peciflc } \\
\text { Ocean }\end{array}$ & & 24 & & - & & & \\
\hline
\end{tabular}

Parasitic Animals:

lots: 2 squid beaks

Notes on Squid:

digested remains

Notes on Fish:

General Notes:

Digested remains beyond identification.

PACIFIC OCEANOGRAPHIC BIOLOGICAL SURVEY PROGRAM

SMITHSONIAN OCEANOGRAPHIC SORTING CENTER—SMITHSONIAN INSTITUTION

S1-USNM-830

P. Grishan $8 / 1 / 66$

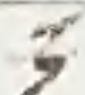

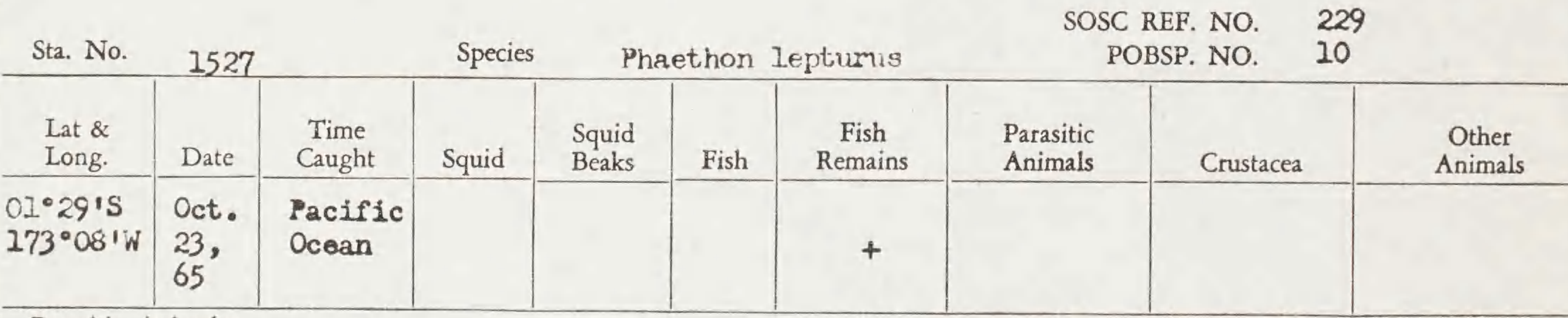

Parasitic Animals:

Lots: 1 fish remains

Notes on Squid:

Notes on Fish:

Fish remains with bones.

General Notes:

PACIFIC OCEANOGRAPHIC BIOLOGICAL SURVEY PROGRAM

SMITHSONIAN OCEANOGRAPHIC SORTING CENTER—SMITHSONIAN INSTITUTION

S1-USNM-Q30

7.65

P. Grisham 8/10/66 
SOSC REF. NO. 229

\begin{tabular}{c|c} 
Sta. No. & 1531 \\
\begin{tabular}{c|c} 
Lat \& \\
Long.
\end{tabular} & Date \\
\hline $01 \cdot 52^{\prime}$ 'S & Oct. \\
$172^{\circ} 53^{\prime} \mathrm{W}$ & $\begin{array}{l}23, \\
65\end{array}$ \\
&
\end{tabular}

POBSP. NO. 10

Parasitic Animals:

Iots: 2 Pish

Notes on Squid:

fish remains

Notes on Fish:

1. Very small.

2. Heads of two digested.

General Notes:

PACIFIC OCEANOGRAPHIC BIOLOGICAL SURVEY PROGRAM

SMITHSONIAN OCEANOGRAPHIC SORTING CENTER_SMITHSONIAN INSTITUTION

P. Grisham 8/9/66

SOSC REF. NO. 229

POBSP. NO. 10

\begin{tabular}{c|c|c|c|c|c|c|} 
Sta. No. & \multicolumn{1}{c}{1542} & & Species & Sula sula & \\
\hline $\begin{array}{c}\text { Lat \& } \\
\text { Long. }\end{array}$ & Date & $\begin{array}{l}\text { Time } \\
\text { Caught }\end{array}$ & Squid & $\begin{array}{c}\text { Squid } \\
\text { Beaks }\end{array}$ & Fish & $\begin{array}{c}\text { Fish } \\
\text { Remains }\end{array}$ \\
\hline $\begin{array}{l}00^{\circ} 17^{\prime} \text { 'S } \\
167^{\circ} 42^{\prime} \text { 'W }\end{array}$ & $\begin{array}{l}\text { Oct. } \\
25, \\
65\end{array}$ & $\begin{array}{l}\text { Pacific } \\
\text { Ocean }\end{array}$ & 1 & 38 & & + \\
\end{tabular}

Parasitic Animals:

lots: 5 squid beaks squid

Notes on Squid: 1. Head digested.

2. Squid remains with tentacles.

squid remains

fish remains

eyeballs (9)

Notes on Fish:

General Notes:

PACIFIC OCEANOGRAPHIC BIOLOGICAL SURVEY PROGRAM SMITHSONIAN OCEANOGRAPHIC SORTING CENTER_-SMITHSONIAN INSTITUTION 
SOSC REF. NO. 2297

\begin{tabular}{|c|c|c|c|c|c|c|c|c|c|}
\hline Sta. No. & 2547 & & Speci & Sula & ctyl & & & P. NO. 10 & \\
\hline $\begin{array}{l}\text { Lat \& } \\
\text { Long. }\end{array}$ & Date & $\begin{array}{l}\text { Time } \\
\text { Caught }\end{array}$ & Squid & $\begin{array}{l}\text { Squid } \\
\text { Beaks }\end{array}$ & Fish & $\begin{array}{c}\text { Fish } \\
\text { Remains }\end{array}$ & $\begin{array}{l}\text { Parasitic } \\
\text { Animals }\end{array}$ & Crustacea & $\begin{array}{l}\text { Other } \\
\text { Animals }\end{array}$ \\
\hline $\begin{array}{l}0^{\circ} 07^{\prime S} \\
164^{\circ} 53^{1} \mathrm{~W}\end{array}$ & $\begin{array}{l}\text { Oct. } \\
26, \\
65\end{array}$ & $\begin{array}{l}\text { Pacific } \\
\text { Ocean }\end{array}$ & & 1 & & - & & $x$ & \\
\hline
\end{tabular}

Parasitic Animals:

lots: 2 digested remains

Notes on Squid: squid beaks

Notes on Fish:

General Notes:

Digested remains with bits of wood.

PACIFIC OCEANOGRAPHIC BIOLOGICAL SURVEY PROGRAM SMITHSONIAN OCEANOGRAPHIC SORTING CENTER—SMITHSONIAN INSTITUTION

S1.USNM - -30

P. Grisham 8/1/66

T.65 5

\begin{tabular}{c|l} 
Sta. No. & 1548 \\
\hline \begin{tabular}{c|c} 
Lat \& \\
Long.
\end{tabular} & Date \\
\hline $00^{\circ} 07^{\prime}$ 's & Oct. \\
$164^{\circ} 53^{\prime}$ 'W & $\begin{array}{l}26, \\
65\end{array}$ \\
\hline
\end{tabular}

Parasitic Animals:

Notes on Squid:

\begin{tabular}{|} 
Time \\
Caught \\
\hline $\begin{array}{l}\text { Pacific } \\
\text { Ocean }\end{array}$ \\
\hline
\end{tabular}

Species

Sula dactylatra

\begin{tabular}{|c|c|c|c|} 
Squid & $\begin{array}{c}\text { Squid } \\
\text { Beaks }\end{array}$ & Fish & $\begin{array}{c}\text { Fish } \\
\text { Remains }\end{array}$ \\
\hline & & 1 & + \\
& & &
\end{tabular}

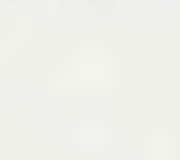

Notes on Fish:

Fish in fair condition.

General Notes:
SOSC REF. NO. 229

POBSP. NO. 10

\begin{tabular}{|r|r|r}
$\begin{array}{r}\text { Parasitic } \\
\text { Animals }\end{array}$ & Crustacea & $\begin{array}{c}\text { Other } \\
\text { Animals }\end{array}$ \\
\hline $\begin{array}{r}\text { Nematode } \\
\text { (1) }\end{array}$ & &
\end{tabular}

lots: 3 fish

fish remains

nematode 
SOSC REF. NO. 229

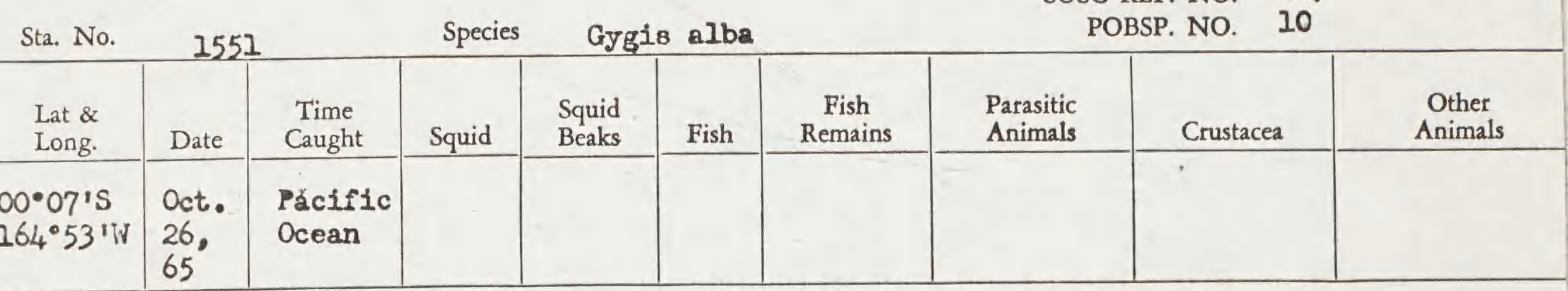

Parasitic Animals:

Notes on Squid:

Notes on Fish:

General Notes:

The specimen was not received.

PACIFIC OCEANOGRAPHIC BIOLOGICAL SURVEY PROGRAM

SMITHSONIAN OCEANOGRAPHIC SORTING CENTER—SMITHSONIAN INSTITUTION

$51-$ USNM-830
$7-65$

P. Grisham $9 / 16 / 66$

$7-65$

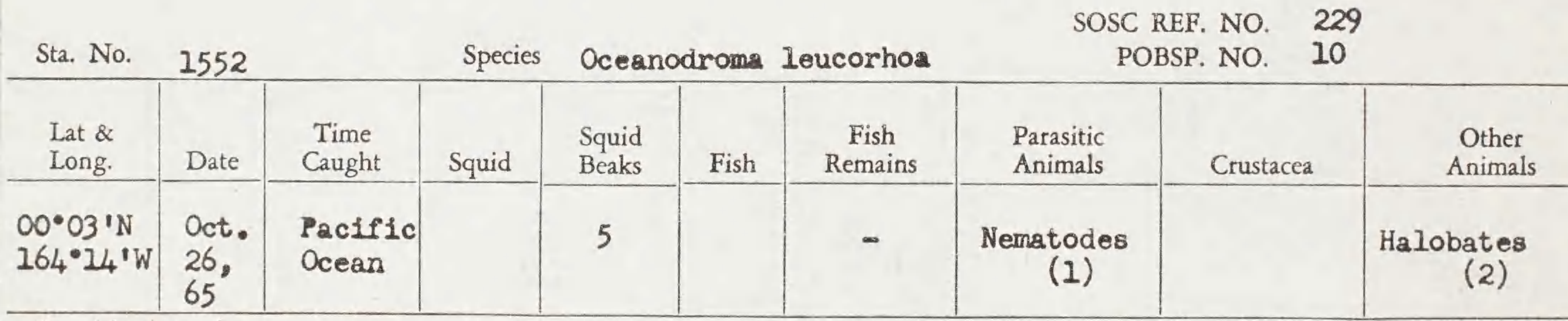

Parasitic Animals:

Lots: 5 nematodes

Notes on Squid:

halobates

squid beaks

cotton

Notes on Fish:

digested remains

General Notes:

Digested remains with a few halobate fragments.

PACIFIC OCEANOGRAPHIC BIOLOGICAL SURVEY PROGRAM

S1-UBNAM- 30

SMITHSONIAN OCEANOGRAPHIC SORTING CENTER-SMITHSONIAN INSTITUTION
P. Grisham $8 / 8 / 66$ 
SOSC REF. NO. 229

\begin{tabular}{|c|c|}
\hline $\begin{array}{l}\text { Lat \& } \\
\text { Long. }\end{array}$ & Date \\
\hline $\begin{array}{l}6^{\circ} 47^{\prime} \mathrm{N} \\
57^{\circ} 29^{\prime} \mathrm{W}\end{array}$ & $\begin{array}{l}\text { Nov. } \\
1, \\
65\end{array}$ \\
\hline
\end{tabular}

Species Oceanodroma Ioucorhoa

POBSP. NO. 10

Parasitic Animals:

lots: 1 digested remains

Notes on Squid:

Notes on Fish:

General Notes:

Digested remains beyond identification.

PACIFIC OCEANOGRAPHIC BIOLOGICAL SURVEY PROGRAM

SMITHSONIAN OCEANOGRAPHIC SORTING CENTER_SMITHSONIAN/INSTITUTION

S1-USNM-830

$7-68$

\section{5}

SOSC REF. NO. 229

\begin{tabular}{|c|c|c|c|c|c|c|c|c|c|}
\hline Sta. No. & 157 & & Species & Pter & roma & alba & & NO. & \\
\hline $\begin{array}{l}\text { Lat \& } \\
\text { Long. }\end{array}$ & Date & $\begin{array}{c}\text { Time } \\
\text { Caught }\end{array}$ & Squid & $\begin{array}{l}\text { Squid } \\
\text { Beaks }\end{array}$ & Fish & $\begin{array}{c}\text { Fish } \\
\text { Remains }\end{array}$ & $\begin{array}{l}\text { Parasitic } \\
\text { Animals }\end{array}$ & Crustacea & $\begin{array}{c}\text { Other } \\
\text { Animals }\end{array}$ \\
\hline $\begin{array}{l}06^{\circ} 53^{\prime} \mathrm{N} \\
257^{\circ} 27^{\prime} \mathrm{W}\end{array}$ & $\begin{array}{l}\text { Nov. } \\
1, \\
65\end{array}$ & $\begin{array}{l}\text { Pacific } \\
\text { Ocean }\end{array}$ & & 180 & & - & & & \\
\hline
\end{tabular}

Parasitic Animals:

Iots: 2 digested remains

Notes on Squid: squid beaks

Notes on Fish:

General Notes:

Digested remains with squid beak fragments.

PACIFIC OCEANOGRAPHIC BIOLOGICAL SURVEY PROGRAM

SMITHSONIAN OCEANOGRAPHIC SORTING CENTER—SMITHSONIAN INSTITUTION 
SOSC REF. NO. 229

\begin{tabular}{|c|c|c|c|c|c|c|c|c|c|}
\hline $\begin{array}{l}\text { Lat \& } \\
\text { Long. }\end{array}$ & Date & $\begin{array}{l}\text { Time } \\
\text { Caught }\end{array}$ & Squid & $\begin{array}{l}\text { Squid } \\
\text { Beaks }\end{array}$ & Fish & $\begin{array}{c}\text { Fish } \\
\text { Remains }\end{array}$ & $\begin{array}{l}\text { Parasitic } \\
\text { Animals }\end{array}$ & Crustacea & $\begin{array}{c}\text { Other } \\
\text { Animals }\end{array}$ \\
\hline $\begin{array}{l}07^{\circ} 05^{\prime} \mathrm{N} \\
257^{\circ} 28^{\prime} \mathrm{W}\end{array}$ & $\begin{array}{l}\text { Nov. } \\
1, \\
65\end{array}$ & $\begin{array}{l}\text { Pacific } \\
\text { Ocean }\end{array}$ & 1 & 7 & 2 & + & & & \\
\hline
\end{tabular}

Parasitic Animals:

lots: 5 squid beaks

Notes on Squid:

Head digested.

squid

IIsh

fish remains

Notes on Fish: 1. Fish remains with bones.

eyeballs (1)

2. Heads digested.

General Notes:

PACIFIC OCEANOGRAPHIC BIOLOGICAL SURVEY PROGRAM SMITHSONIAN OCEANOGRAPHIC SORTING CENTER—SMITHSONIAN INSTITUTION

S1-USNM-B30

P. Grisham 8/16/66

z

Sta. No. $1572 \quad$ Species Pterodroma hypoleuca

SOSC REF. NO. 229

\begin{tabular}{|c|c|c|c|c|c|c|c|c|c|}
\hline $\begin{array}{l}\text { Lat \& } \\
\text { Long. }\end{array}$ & Date & $\begin{array}{c}\text { Time } \\
\text { Caught }\end{array}$ & Squid & $\begin{array}{l}\text { Squid } \\
\text { Beaks }\end{array}$ & Fish & $\begin{array}{c}\text { Fish } \\
\text { Remains }\end{array}$ & $\begin{array}{l}\text { Parasitic } \\
\text { Animals }\end{array}$ & Crustacea & $\begin{array}{c}\text { Other } \\
\text { Animals }\end{array}$ \\
\hline $\begin{array}{l}07^{\circ} 11^{\prime} \text { iv } \\
157^{\circ} 27^{\circ}\end{array}$ & $\begin{array}{l}\text { Nor. } \\
1, \\
65\end{array}$ & $\begin{array}{l}\text { Pacifid } \\
\text { Ocean }\end{array}$ & & 9 & & - & & & \\
\hline
\end{tabular}

Parasitic Animals:

Notes on Squid:

lots: 3 squid beaks

eyeballs (13)

digested remains

Notes on Fish:

General Notes:

Digested remains with a few squid boak fragments.

PACIFIC OCEANOGRAPHIC BIOLOGICAL SURVEY PROGRAM

SMITHSONIAN OCEANOGRAPHIC SORTING CENTER—SMITHSONIAN INSTITUTION

P. Grisham 9/12/66 
SOSC REF. NO. 229

Sta. No. 1573 Species Pterodroma externa POBSP. NO. 10

\begin{tabular}{|c|c|c|c|c|c|c|c|c|c|}
\hline $\begin{array}{l}\text { Lat \& } \\
\text { Long. }\end{array}$ & Date & $\begin{array}{l}\text { Time } \\
\text { Caught }\end{array}$ & Squid & $\begin{array}{l}\text { Squid } \\
\text { Beaks }\end{array}$ & Fish & $\begin{array}{c}\text { Fish } \\
\text { Remains }\end{array}$ & $\begin{array}{l}\text { Parasitic } \\
\text { Animals }\end{array}$ & Crustacea & $\begin{array}{c}\text { Other } \\
\text { Animals }\end{array}$ \\
\hline $\begin{array}{l}\mathrm{H}^{\circ} 08^{\prime} \mathrm{N} \\
15^{\circ} 39^{\prime} \mathrm{W}\end{array}$ & $\begin{array}{l}\text { Nov. } \\
3, \\
65\end{array}$ & $\begin{array}{l}\text { Pacific } \\
\text { Ocean }\end{array}$ & & & & - & & & \\
\hline
\end{tabular}

Parasitic Animals:

Lots: 1 digested remains

Notes on Squid:

Notes on Fish:

General Notes:

Digested remains beyond identification.

PACIFIC OCEANOGRAPHIC BIOLOGICAL SURVEY PROGRAM

SMITHSONIAN OCEANOGRAPHIC SORTING CENTER—SMITHSONIAN INSTITUTION

P. Grisham 8/1/66

S1-USNM-E30

7-65

$$
5
$$

\begin{tabular}{|c|c|c|c|c|c|c|c|c|c|}
\hline Sta. No. & 1574 & & Speci & Pte & roma & ypol euca & & $\begin{array}{l}\text { NO. } \\
\text { NO. }\end{array}$ & \\
\hline $\begin{array}{l}\text { Lat \& } \\
\text { Long. }\end{array}$ & Date & $\begin{array}{c}\text { Time } \\
\text { Caught }\end{array}$ & Squid & $\begin{array}{l}\text { Squid } \\
\text { Beaks }\end{array}$ & Fish & $\begin{array}{c}\text { Fish } \\
\text { Remains }\end{array}$ & $\begin{array}{l}\text { Parasitic } \\
\text { Animals }\end{array}$ & Crustacea & $\begin{array}{c}\text { Other } \\
\text { Animals }\end{array}$ \\
\hline $\begin{array}{l}14^{\circ} 1 I^{\prime} \mathrm{N} \\
157^{\circ} 38^{\prime} \mathrm{W}\end{array}$ & $\begin{array}{l}\text { Nor. } \\
3, \\
65\end{array}$ & $\begin{array}{l}\text { Pacific } \\
\text { Ocean }\end{array}$ & & 10 & & - & & & \\
\hline
\end{tabular}

Parasitic Animals:

Iots: 2 squid beaks

Notes on Squid: digested remains

Notes on Fish:

General Notes:

Digested remains beyond identification.

PACIFIC OCEANOGRAPHIC BIOLOGICAL SURVEY PROGRAM SMITHSONIAN OCEANOGRAPHIC SORTING CENTER — SMITHSONIAN INSTITUTION 
SOSC REF. NO. 229

\begin{tabular}{|c|c|c|c|c|c|c|c|c|c|}
\hline Sta. No. & 1575 & & Species & Pter & oma & oleuca & & NO. & \\
\hline $\begin{array}{l}\text { Lat \& } \\
\text { Long. }\end{array}$ & Date & $\begin{array}{c}\text { Time } \\
\text { Caught }\end{array}$ & Squid & $\begin{array}{l}\text { Squid } \\
\text { Beaks }\end{array}$ & Fish & $\begin{array}{c}\text { Fish } \\
\text { Remains }\end{array}$ & $\begin{array}{l}\text { Parasitic } \\
\text { Animals }\end{array}$ & Crustacea & $\begin{array}{c}\text { Other } \\
\text { Animals }\end{array}$ \\
\hline $\begin{array}{l}18^{\circ} 46^{\prime} \mathrm{N} \\
157^{\circ} 48^{\prime} \mathrm{W}\end{array}$ & $\begin{array}{l}\text { Nov. } \\
4, \\
65\end{array}$ & $\begin{array}{l}\text { Pacific } \\
\text { Ocean }\end{array}$ & & & & - & $\begin{array}{c}\text { Nematodes } \\
\text { (1) }\end{array}$ & & \\
\hline
\end{tabular}

Parasitic Animals:

1ots: 3 squid remains

Notes on Squid:

Tentacles of squids with suckers visible.

nematodes digested remains

Notes on Fish:

General Notes:

Digested remains with pieces of wood.

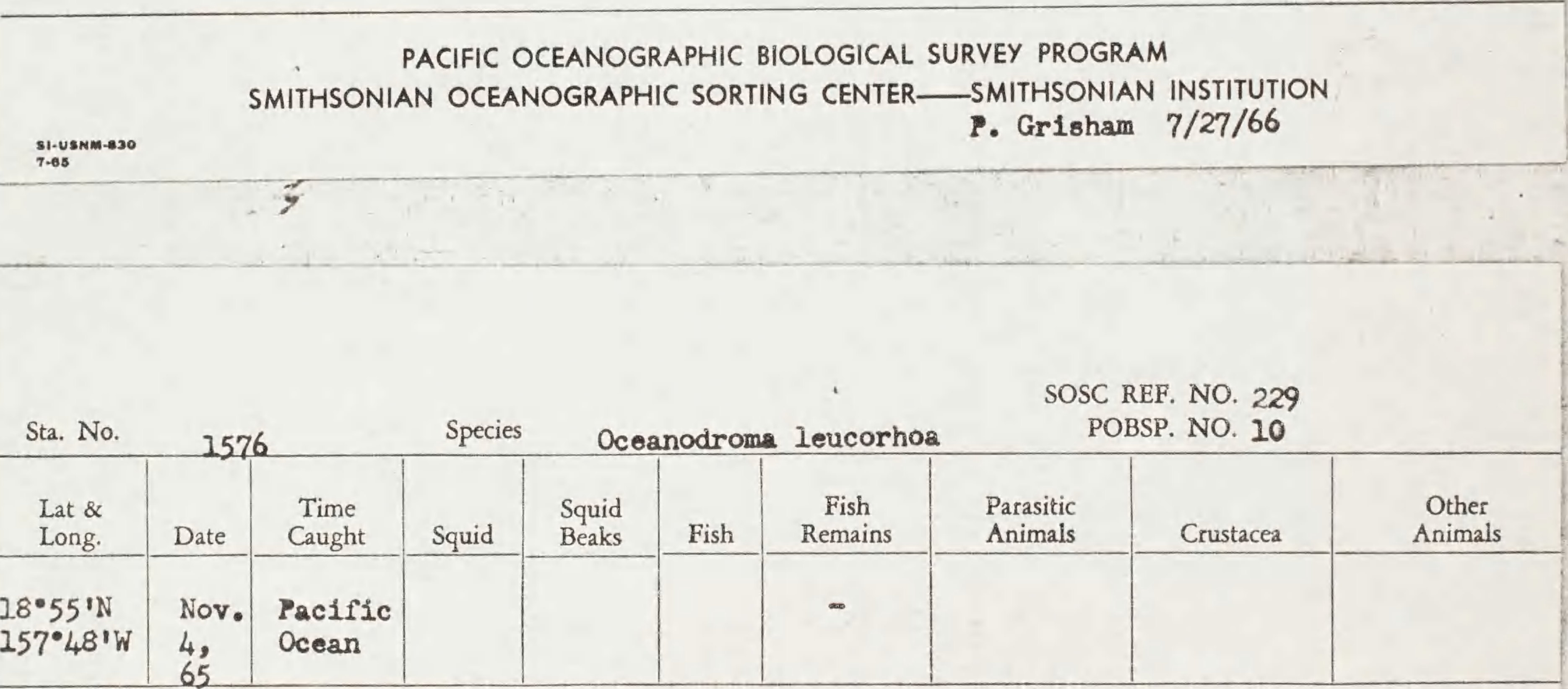

Parasitic Animals:

Notes on Squid:

10ts: I difested remains

Notes on Fish:

General Notes:

Digested remains beyond identification.

PACIFIC OCEANOGRAPHIC BIOLOGICAL SURVEY PROGRAM

SMITHSONIAN OCEANOGRAPHIC SORTING CENTER—SMITHSONIAN INSTITUTION

P. Grisham 8/2/66 


\begin{tabular}{|c|c|c|c|c|c|c|c|c|c|}
\hline Sta. No. & & & Speci & & $38 t$ & dus & & $\begin{array}{l}\text { NO. } \\
\text { NO. }\end{array}$ & \\
\hline \multirow[t]{2}{*}{$\begin{array}{l}\text { Lat \& } \\
\text { Long. }\end{array}$} & Date & $\begin{array}{c}\text { Time } \\
\text { Caught }\end{array}$ & Squid & $\begin{array}{l}\text { Squid } \\
\text { Beaks }\end{array}$ & Fish & $\begin{array}{c}\text { Fish } \\
\text { Remains }\end{array}$ & $\begin{array}{l}\text { Parasitic } \\
\text { Animals }\end{array}$ & Cxustacea & $\begin{array}{c}\text { Other } \\
\text { Animals }\end{array}$ \\
\hline & $\begin{array}{l}\text { June } \\
1, \\
65\end{array}$ & $\begin{array}{l}\text { Phoenix } \\
\text { I's Lands }\end{array}$ & & & & & & & \\
\hline
\end{tabular}

Notes on Squid:

Notes on Fish:

General Notes:

The specimen was not received.

PACIFIC OCEANOGRAPHIC BIOLOGICAL SURVEY PROGRAM SMITHSONIAN OCEANOGRAPHIC SORTING CENTER — SMITHSONIAN INSTITUTION

P. Grisham 9/19/66

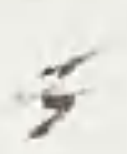

SOSC REF. NO. 229

\begin{tabular}{l|c|c|} 
Sta. No. & \multicolumn{2}{|c|}{5756} \\
\begin{tabular}{c|c} 
Lat \& \\
Long.
\end{tabular} & Date & $\begin{array}{c}\text { Time } \\
\text { Caught }\end{array}$ \\
\hline & $\begin{array}{l}\text { Sept. } \\
16, \\
65\end{array}$ & $\begin{array}{l}\text { Howland } \\
\text { Is land }\end{array}$ \\
\hline
\end{tabular}

Species Sula dactylatra POBSP. NO. 10

Parasitic Animals:

Iots:,2 squid beaks

Notes on Squid: digested remains

Notes on Fish:

General Notes:

Digested remains beyond identification.

PACIFIC OCEANOGRAPHIC BIOLOGICAL SURVEY PROGRAM

SMITHSONIAN OCEANOGRAPHIC SORTING CENTER—SMITHSONIAN INSTITUTION

P. Grisham $8 / 24 / 66$ 


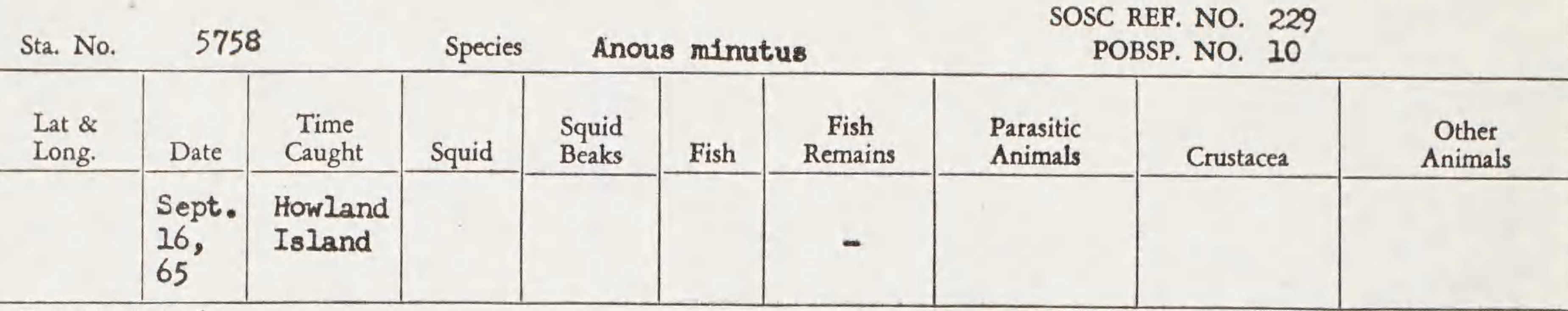

Parasitic Animals:

Notes on Squid:

1ats: 1 digasted remains

Notes on Fish:

General Notes:

Digested remains beyond Identification.

PACIFIC OCEANOGRAPHIC BIOLOGICAL SURVEY PROGRAM

SMITHSONIAN OCEANOGRAPHIC SORTING CENTER_SMITHSONIAN INSTITUTION

S1-USNM-830

P. Grisham 8/16/66

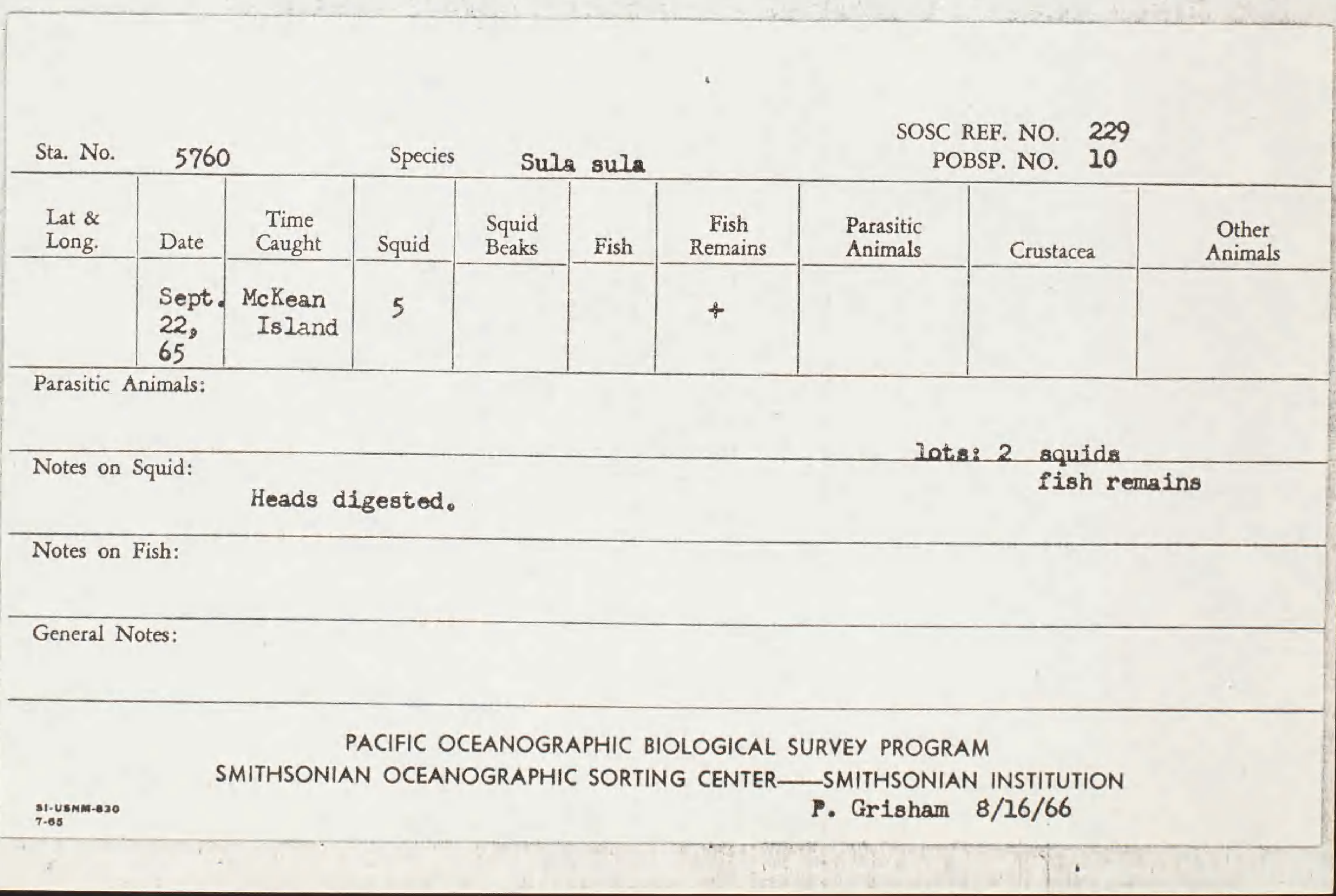




\begin{tabular}{|c|c|c|c|c|c|c|c|c|c|}
\hline \multirow{2}{*}{$\begin{array}{l}\text { Sta. No. } \\
\text { Lat \& } \\
\text { Long. }\end{array}$} & \multicolumn{2}{|c|}{5764} & Species & \multicolumn{3}{|c|}{ Phathon rubricauda } & \multicolumn{2}{|c|}{$\begin{array}{l}\text { SOSC REF. NO. } \\
\text { POBSP. NO. }\end{array}$} & $\begin{array}{l}229 \\
10\end{array}$ \\
\hline & Date & $\begin{array}{l}\text { Time } \\
\text { Caught }\end{array}$ & Squid & $\begin{array}{l}\text { Squid } \\
\text { Beaks }\end{array}$ & Fish & $\begin{array}{c}\text { Fish } \\
\text { Remains }\end{array}$ & $\begin{array}{l}\text { Parasitic } \\
\text { Animals }\end{array}$ & Crustacea & $\begin{array}{c}\text { Other } \\
\text { Animals }\end{array}$ \\
\hline & $\begin{array}{l}\text { Sept. } \\
23 \text {, } \\
65\end{array}$ & $\begin{array}{l}\text { Mckean } \\
\text { Island }\end{array}$ & - & 10 & & - & & & \\
\hline
\end{tabular}

Parasitic Animals:

Notes on Squid:

lots: 2 squid beaks digested remains

Notes on Fish:

General Notes:

Digested remains beyond identification.

PACIFIC OCEANOGRAPHIC BIOLOGICAL SURVEY PROGRAM

SMITHSONIAN OCEANOGRAPHIC SORTING CENTER—SMITHSONIAN INSTITUTION

S1-USNM- -330

P. Grisham 8/1/66

\begin{tabular}{|c|c|c|c|c|c|c|c|c|c|}
\hline Sta. No. & 576 & & Specie & & egat & ariel & \multicolumn{3}{|c|}{$\begin{aligned} \text { SOSC REF. NO. } & 229 \\
\text { POBSP. NO. } & 10\end{aligned}$} \\
\hline $\begin{array}{l}\text { Lat \& } \\
\text { Long. }\end{array}$ & Date & $\begin{array}{l}\text { Time } \\
\text { Caught }\end{array}$ & Squid & $\begin{array}{l}\text { Squid } \\
\text { Beaks }\end{array}$ & Fish & $\begin{array}{c}\text { Fish } \\
\text { Remains }\end{array}$ & $\begin{array}{l}\text { Parasitic } \\
\text { Animals }\end{array}$ & Crustacea & $\begin{array}{l}\text { Other } \\
\text { Animals }\end{array}$ \\
\hline & $\begin{array}{l}\text { Sept. } \\
23, \\
65\end{array}$ & $\begin{array}{l}\text { McKean } \\
\text { Island }\end{array}$ & & 24 & & - & & & \\
\hline
\end{tabular}

Parasitic Animals:

Notes on Squid:

Lots: 3 squid beaks eyeballs (9) digested remains

\section{Notes on Fish:}

General Notes:

Digested remains beyond 1dentification but with bits of wood.

PACIFIC OCEANOGRAPHIC BIOLOGICAL SURVEY PROGRAM

SMITHSONIAN OCEANOGRAPHIC SORTING CENTER—SMITHSONIAN INSTITUTION 
SOSC REF. NO. 229

\begin{tabular}{l|l|l|l|l|l|l|l|l|l|} 
Sta. No. & 5766 & \multicolumn{2}{c}{ Species } & \multicolumn{2}{c}{ Sula dactylatra } & \multicolumn{2}{c}{ POBSP. NO. } & 10 \\
$\begin{array}{l}\text { Lat \& } \\
\text { Long. }\end{array}$ & Date & $\begin{array}{c}\text { Time } \\
\text { Caught }\end{array}$ & Squid & $\begin{array}{l}\text { Squid } \\
\text { Beaks }\end{array}$ & Fish & $\begin{array}{c}\text { Fish } \\
\text { Remains }\end{array}$ & $\begin{array}{c}\text { Parasitic } \\
\text { Animals }\end{array}$ & Crustacea & $\begin{array}{c}\text { Other } \\
\text { Animals }\end{array}$ \\
\hline & $\begin{array}{l}\text { Sept. } \\
23, \\
65\end{array}$ & $\begin{array}{c}\text { McKean } \\
\text { Island }\end{array}$ & & 76 & & - & $\begin{array}{c}\text { Nematode } \\
\text { (1) }\end{array}$ & & \\
\hline
\end{tabular}

Parasitic Animals:

1ots: 3 digested remains

Notes on Squid: squid beaks nematodes

Notes on Fish:

General Notes:

PACIFIC OCEANOGRAPHIC BIOLOGICAL SURVEY PROGRAM SMITHSONIAN OCEANOGRAPHIC SORTING CENTER — SMITHSONIAN INSTITUTION

S1-USNM- 200
7.65

P. Grisham 8/22/66

Sta. No.

Species Sula dactylatra

SOSC REF. NO. 229

\begin{tabular}{|c|c|c|c|c|c|c|c|c|c|}
\hline $\begin{array}{l}\text { Lat \& } \\
\text { Long. }\end{array}$ & Date & $\begin{array}{l}\text { Time } \\
\text { Caught }\end{array}$ & Squid & $\begin{array}{l}\text { Squid } \\
\text { Beaks }\end{array}$ & Fish & $\begin{array}{c}\text { Fish } \\
\text { Remains }\end{array}$ & $\begin{array}{l}\text { Parasitic } \\
\text { Animals }\end{array}$ & Crustacea & $\begin{array}{l}\text { Other } \\
\text { Animals }\end{array}$ \\
\hline & $\begin{array}{l}\text { Sept. } \\
23 \text {, } \\
65\end{array}$ & $\begin{array}{l}\text { McKean } \\
\text { Island }\end{array}$ & & 47 & & - & & & \\
\hline
\end{tabular}

Parasitic Animals:

Lots: 3 eyeballs (6)

Notes on Squid:

squid beaks

digested remains

Notes on Fish:

General Notes:

PACIFIC OCEANOGRAPHIC BIOLOGICAL SURVEY PROGRAM

SMITHSONIAN OCEANOGRAPHIC SORTING CENTER—SMITHSONIAN INSTITUTION

P. Grisham $8 / 24 / 66$ 


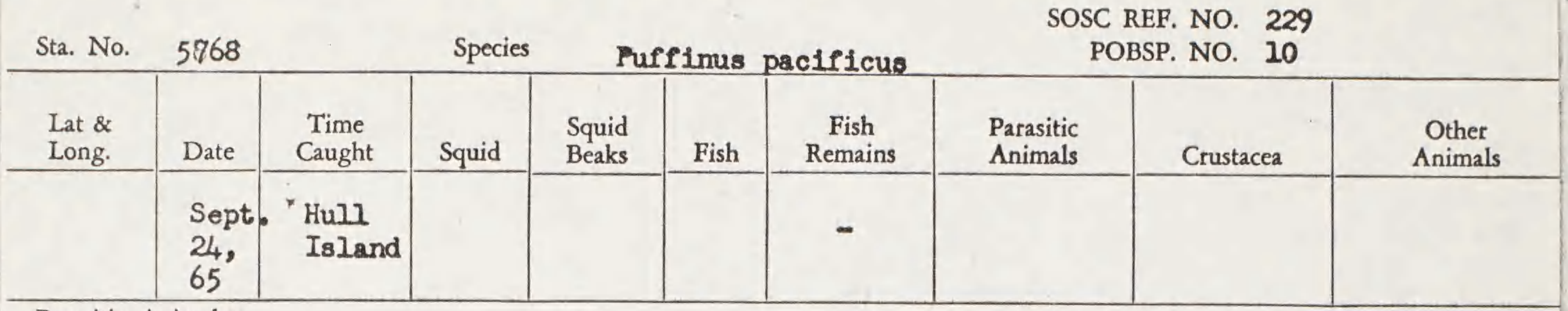

Parasitic Animals:

Lots: 1 digested remains

Notes on Squid:

Notes on Fish:

General Notes:

Digested remains beyond identification.

PACIFIC OCEANOGRAPHIC BIOLOGICAL SURVEY PROGRAM

SMITHSONIAN OCEANOGRAPHIC SORTING CENTER—SMITHSONIAN INSTITUTION

SI-USNM-830

P. Grisham 7/27/66

SOSC REF. NO. 229

\begin{tabular}{|c|c|c|c|c|c|c|c|c|c|}
\hline Sta. No. & & & Species & Het & sscel & Incanum & & NO. & \\
\hline \multirow[t]{2}{*}{$\begin{array}{l}\text { Lat \& } \\
\text { Long. }\end{array}$} & Date & $\begin{array}{l}\text { Time } \\
\text { Caught }\end{array}$ & Squid & $\begin{array}{l}\text { Squid } \\
\text { Beaks }\end{array}$ & Fish & $\begin{array}{c}\text { Fish } \\
\text { Remains }\end{array}$ & $\begin{array}{l}\text { Parasitic } \\
\text { Animals }\end{array}$ & Crustacea & $\begin{array}{c}\text { Other } \\
\text { Animals }\end{array}$ \\
\hline & $\begin{array}{l}\text { Sept. } \\
30 \\
65\end{array}$ & $\begin{array}{l}\text { Phoenix } \\
\text { Island }\end{array}$ & & & & - & & & \\
\hline
\end{tabular}

Parasitic Animals:

10ts: 1 insect remains

Notes on Squid:

Notes on Fish:

General Notes:

Insect remaing with rocks.

PACIFIC OCEANOGRAPHIC BIOLOGICAL SURVEY PROGRAM SMITHSONIAN OCEANOGRAPHIC SORTING CENTER — SMITHSONIAN INSTITUTION 
SOSC REF. NO. 229

\begin{tabular}{l|c|c|c|c|c|c|c|c} 
Sta. No. & 5770 & \multicolumn{2}{c}{ Species } & Erolia melanotos & \multicolumn{2}{c}{ POBSP. NO. 10} \\
\hline $\begin{array}{l}\text { Lat \& } \\
\text { Long. }\end{array}$ & $\begin{array}{c}\text { Date } \\
\text { Time } \\
\text { Caught }\end{array}$ & Squid & $\begin{array}{c}\text { Squid } \\
\text { Beaks }\end{array}$ & Fish & $\begin{array}{c}\text { Fish } \\
\text { Remains }\end{array}$ & $\begin{array}{c}\text { Parasitic } \\
\text { Animals }\end{array}$ & Crustacea & $\begin{array}{c}\text { Other } \\
\text { Animals }\end{array}$ \\
\hline $\begin{array}{l}\text { Sept. } \\
30, \\
65\end{array}$ & $\begin{array}{c}\text { Phoenix } \\
\text { Island }\end{array}$ & & & & & & & \\
\end{tabular}

Parasitic Animals:

lots: 1 insect grub

Notes on Squid:

Notes on Fish:

General Notes:

PACIFIC OCEANOGRAPHIC BIOLOGICAL SURVEY PROGRAM SMITHSONIAN OCEANOGRAPHIC SORTING CENTER—SMITHSONIAN INSTITUTION

S1-USNM-630

P. Grisham $8 / 2 / 66$

5

Sta. No.

\begin{tabular}{|c|c|c|c|c|c|c|c|c|c|}
\hline $\begin{array}{l}\text { Lat \& } \\
\text { Long. }\end{array}$ & Date & $\begin{array}{l}\text { Time } \\
\text { Caught }\end{array}$ & Squid & $\begin{array}{l}\text { Squid } \\
\text { Beaks }\end{array}$ & Fish & $\begin{array}{c}\text { Fish } \\
\text { Remains }\end{array}$ & $\begin{array}{l}\text { Parasitic } \\
\text { Animals }\end{array}$ & Crustacea & $\begin{array}{l}\text { Other } \\
\text { Animals }\end{array}$ \\
\hline & $\begin{array}{l}\text { Sept. } \\
30 \text {, } \\
65\end{array}$ & $\begin{array}{l}\text { Phoenix } \\
\text { Island }\end{array}$ & & 2 & & $\infty$ & & & \\
\hline
\end{tabular}

Species

Sula leucogaster

Parasitic Animals:

Lots: 2 digested remains

Notes on Squid: squid beaks

Notes on Fish:

General Notes:

Digested remains beyond identification.

SOSC REF, NO. 229

POBSP. NO. 10 
SOSC REF. NO.

\begin{tabular}{|c|c|c|c|c|c|c|c|c|c|}
\hline $\begin{array}{l}\text { Lat \& } \\
\text { Long. }\end{array}$ & Date & $\begin{array}{c}\text { Time } \\
\text { Caught }\end{array}$ & Squid & $\begin{array}{l}\text { Squid } \\
\text { Beaks }\end{array}$ & Fish & $\begin{array}{c}\text { Fish } \\
\text { Remains }\end{array}$ & $\begin{array}{l}\text { Parasitic } \\
\text { Animals }\end{array}$ & Crustacea & $\begin{array}{l}\text { Other } \\
\text { Animals }\end{array}$ \\
\hline & $\begin{array}{l}\text { Sept. } \\
30 \text {, } \\
65\end{array}$ & $\begin{array}{l}\text { Phoenix } \\
\text { Island }\end{array}$ & & & 2 & - & & & \\
\hline
\end{tabular}

Parasitic Animals:

lots: 1 fish

Notes on Squid:

Notes on Fish:

Heads digested.

General Notes:

No stomach, only stomach contents.

PACIFIC OCEANOGRAPHIC BIOLOGICAL SURVEY PROGRAM

SMITHSONIAN OCEANOGRAPHIC SORTING CENTER — SMITHSONIAN INSTITUTION

P. Grisham 8/22/66

S1. USNM-830

$\operatorname{lin}_{7-65}^{1.05}$

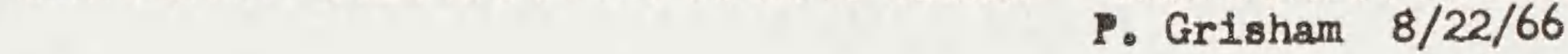

\section{$\xi$}

Sta. No.

Species

Erolia melanotos

SOSC REF. NO. 229

POBSP. NO. 10

\begin{tabular}{|c|c|c|c|c|c|c|c|c|c|}
\hline $\begin{array}{l}\text { Lat \& } \\
\text { Long. }\end{array}$ & Date & $\begin{array}{c}\text { Time } \\
\text { Caught }\end{array}$ & Squid & $\begin{array}{l}\text { Squid } \\
\text { Beaks }\end{array}$ & Fish & $\begin{array}{c}\text { Fish } \\
\text { Remains }\end{array}$ & $\begin{array}{l}\text { Parasitic } \\
\text { Animals }\end{array}$ & Crustacea & $\begin{array}{l}\text { Other } \\
\text { Animals }\end{array}$ \\
\hline & $\begin{array}{l}\text { Oct. } \\
2, \\
65\end{array}$ & $\begin{array}{l}\text { Enderbury } \\
\text { Island }\end{array}$ & & & & - & & & \\
\hline
\end{tabular}

Parasitic Animals:

lots: 1 insect remains

Notes on Squid:

Notes on Fish:

General Notes:

Insect remains with rocks.

PACIFIC OCEANOGRAPHIC BIOLOGICAL SURVEY PROGRAM

SMITHSONIAN OCEANOGRAPHIC SORTING CENTER—SMITHSONIAN INSTITUTION

P. Grisham 8/3/66 
SOSC REF. NO. 229

Sta. No. 5808 Species Erolla melanotos POBSP. NO. 10

\begin{tabular}{|c|c|c|c|c|c|c|c|c|c|}
\hline $\begin{array}{l}\text { Lat \& } \\
\text { Long. }\end{array}$ & Date & $\begin{array}{l}\text { Time } \\
\text { Caught }\end{array}$ & Squid & $\begin{array}{l}\text { Squid } \\
\text { Beaks }\end{array}$ & Fish & $\begin{array}{c}\text { Fish } \\
\text { Remains }\end{array}$ & $\begin{array}{l}\text { Parasitic } \\
\text { Animals }\end{array}$ & Crustacea & $\begin{array}{c}\text { Other } \\
\text { Animals }\end{array}$ \\
\hline & $\begin{array}{l}\text { Oct. } \\
2, \\
65\end{array}$ & $\begin{array}{l}\text { Enderbury } \\
\text { Island }\end{array}$ & & & & - & & & \\
\hline
\end{tabular}

Parasitic Animals:

10ts: 1 digested remains

Notes on Squid:

Notes on Fish:

General Notes:

Digested remains beyond identification.

PACIFIC OCEANOGRAPHIC BIOLOGICAL SURVEY PROGRAM

SMITHSONIAN OCEANOGRAPHIC SORTING CENTER—SMITHSONIAN INSTITUTION

P. Grisham $8 / 15 / 66$

7.65

\section{5}

\begin{tabular}{|c|c|c|c|c|c|c|c|c|c|}
\hline Sta. No. & 5809 & & Speci & & suls & & & $\begin{array}{l}\text { NO. } \\
\text { NO. }\end{array}$ & \\
\hline \multirow[t]{2}{*}{$\begin{array}{l}\text { Lat \& } \\
\text { Long. }\end{array}$} & Date & $\begin{array}{l}\text { Time } \\
\text { Caught }\end{array}$ & Squid & $\begin{array}{l}\text { Squid } \\
\text { Beaks }\end{array}$ & Fish & $\begin{array}{c}\text { Fish } \\
\text { Remains }\end{array}$ & $\begin{array}{l}\text { Parasitic } \\
\text { Animals }\end{array}$ & Crustacea & $\begin{array}{l}\text { Other } \\
\text { Animals }\end{array}$ \\
\hline & $\begin{array}{l}\text { Oct. } \\
2, \\
65\end{array}$ & $\begin{array}{l}\text { Enderbury } \\
\text { Island }\end{array}$ & & 7 & & + & & & \\
\hline
\end{tabular}

Parasitic Animals:

Notes on Squid:

Lots: 3 flsh remains squid beaks eyebalis (2)

Notes on Fish:

Fish remains with bones.

General Notes: 
SOSC REF. NO. 229

Sta. No. $\quad 5811$

Sterna fuscatea

POBSP. NO. 10

\begin{tabular}{|c|c|c|c|c|c|c|c|c|c|}
\hline $\begin{array}{l}\text { Lat \& } \\
\text { Long. }\end{array}$ & Date & $\begin{array}{l}\text { Time } \\
\text { Caught }\end{array}$ & Squid & $\begin{array}{l}\text { Squid } \\
\text { Beaks }\end{array}$ & Fish & $\begin{array}{c}\text { Fish } \\
\text { Remains }\end{array}$ & $\begin{array}{l}\text { Parasitic } \\
\text { Animals }\end{array}$ & Crustacea & $\begin{array}{c}\text { Other } \\
\text { Animals }\end{array}$ \\
\hline & $\begin{array}{l}\text { Sept. } \\
24, \\
65\end{array}$ & $\begin{array}{l}\text { Hull } \\
\text { Island }\end{array}$ & & & & + & & & \\
\hline
\end{tabular}

Parasitic Animals:

1ots: 1 fish remains

Notes on Squid:

Notes on Fish:

Fish remains with bones.

General Notes:

PACIFIC OCEANOGRAPHIC BIOLOGICAL SURVEY PROGRAM

SMITHSONIAN OCEANOGRAPHIC SORTING CENTER — SMITHSONIAN INSTITUTION

51-USNM-830

P. Grisham 7/27/66

S1-Using
7.65

5

SOSC REF. NO. 229

Sta. No.

5812

Species Sterna fuscata

POBSP. NO. 10

\begin{tabular}{|c|c|c|c|c|c|c|c|c|c|}
\hline $\begin{array}{l}\text { Lat \& } \\
\text { Long. }\end{array}$ & Date & $\begin{array}{c}\text { Time } \\
\text { Caught }\end{array}$ & Squid & $\begin{array}{l}\text { Squid } \\
\text { Beaks }\end{array}$ & Fish & $\begin{array}{c}\text { Fish } \\
\text { Remains }\end{array}$ & $\begin{array}{l}\text { Parasitic } \\
\text { Animals }\end{array}$ & Crustacea & $\begin{array}{l}\text { Other } \\
\text { Animals }\end{array}$ \\
\hline & $\begin{array}{l}\text { Sept. } \\
24, \\
65\end{array}$ & $\begin{array}{l}\text { Hull } \\
\text { Island }\end{array}$ & & & & - & & & \\
\hline
\end{tabular}

Parasitic Animals:

lots: 0

Notes on Squid:

Notes on Fish:

General Notes:

The stomach was empty.

PACIFIC OCEANOGRAPHIC BIOLOGICAL SURVEY PROGRAM

SMITHSONIAN OCEANOGRAPHIC SORTING CENTER_- SMITHSONIAN INSTITUTION
P. Grisham $7 / 27 / 66$ 
Sta. No.

$581_{4}$ Species

Erolia acuminata

Parasitic Animals:

Notes on Squid:

Iots: 0

\begin{tabular}{|c|c|c|c|c|c|c|c|c|c|}
\hline $\begin{array}{l}\text { Lat \& } \\
\text { Long. }\end{array}$ & Date & $\begin{array}{l}\text { Time } \\
\text { Caught }\end{array}$ & Squid & $\begin{array}{l}\text { Squid } \\
\text { Beaks }\end{array}$ & Fish & $\begin{array}{c}\text { Fish } \\
\text { Remains }\end{array}$ & $\begin{array}{l}\text { Parasitic } \\
\text { Animals }\end{array}$ & Crustacea & $\begin{array}{l}\text { Other } \\
\text { Animals }\end{array}$ \\
\hline & $\begin{array}{l}\text { Sept. } \\
18, \\
65\end{array}$ & $\begin{array}{l}\text { Baker } \\
\text { Island }\end{array}$ & & & & - & & & \\
\hline
\end{tabular}

SOSC REF. NO. 229

POBSP. NO. 10

Notes on Fish:

General Notes:

The stomach was empty.

PACIFIC OCEANOGRAPHIC BIOLOGICAL SURVEY PROGRAM

SMITHSONIAN OCEANOGRAPHIC SORTING CENTER_SMITHSONIAN INSTITUTION

S1-USNM-630

F. Grisham $8 / 2 / 66$

\section{5}

SOSC REF. NO. 229

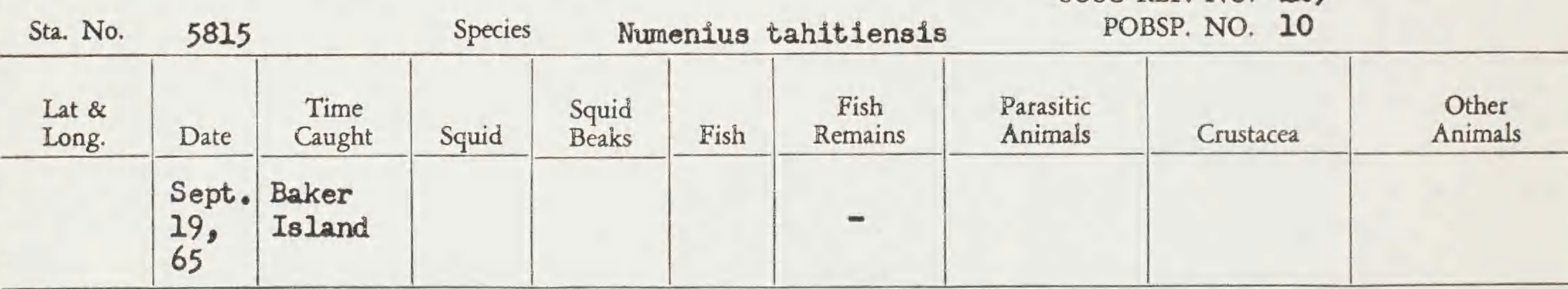

Parasitic Animals:

lots: 1 insect remains

Notes on Squid:

Notes on Fish:

General Notes:

Insect remains with a few stones.

PACIFIC OCEANOGRAPHIC BIOLOGICAL SURVEY PROGRAM

SMITHSONIAN OCEANOGRAPHIC SORTING CENTER—SMITHSONIAN INSTITUTION

81-U⿻上丨ต-830

P. Grisham 8/15/66 
SOSC REF. NO. 229

\begin{tabular}{l|l|} 
Sta. No. & 5816 \\
\begin{tabular}{l|l} 
Lat \& \\
Long.
\end{tabular} & Date \\
\hline & $\begin{array}{l}\text { Sept. } \\
16, \\
65\end{array}$ \\
\hline
\end{tabular}

Species

Heteroscelus incanum

POBSP. NO. 10

Parasitic Animals:

\begin{tabular}{|c|c|c|c|c}
$\begin{array}{c}\text { Time } \\
\text { Caught }\end{array}$ & Squid & $\begin{array}{c}\text { Squid } \\
\text { Beaks }\end{array}$ & Fish & $\begin{array}{c}\text { Fish } \\
\text { Remains }\end{array}$ \\
\hline $\begin{array}{l}\text { Baker } \\
\text { Island }\end{array}$ & & & & - \\
\hline & & &
\end{tabular}

\begin{tabular}{|c|c|c|}
$\begin{array}{c}\text { Parasitic } \\
\text { Animals }\end{array}$ & Crustacea & $\begin{array}{c}\text { Other } \\
\text { Animals }\end{array}$ \\
\hline & Crabs (9) &
\end{tabular}

Iots: 1 crabs

Notes on Squid:

Notes on Fish:

General Notes:

Legs of some are detached.

PACIFIC OCEANOGRAPHIC BIOLOGICAL SURVEY PROGRAM

SMITHSONIAN OCEANOGRAPHIC SORTING CENTER—SMITHSONIAN INSTITUTION

P. Grisham $8 / 2 / 66$

SI-USNM-:30

$7-83$

\section{3}

\begin{tabular}{|c|c|c|c|c|c|c|c|c|c|}
\hline Sta. No. & 5818 & & Speci & & a $1 a$ & nica & & $\begin{array}{l}\text { No. } \\
\text { No. }\end{array}$ & \\
\hline \multirow[t]{2}{*}{$\begin{array}{l}\text { Lat \& } \\
\text { Long. }\end{array}$} & Date & $\begin{array}{c}\text { Time } \\
\text { Caught }\end{array}$ & Squid & $\begin{array}{l}\text { Squid } \\
\text { Beaks }\end{array}$ & Fish & $\begin{array}{c}\text { Fish } \\
\text { Remains }\end{array}$ & $\begin{array}{l}\text { Parasitic } \\
\text { Animals }\end{array}$ & Crustacea & $\begin{array}{l}\text { Other } \\
\text { Animals }\end{array}$ \\
\hline & $\begin{array}{l}\text { Oct. } \\
19, \\
65\end{array}$ & $\begin{array}{l}\text { Baker } \\
\text { Island }\end{array}$ & & & & + & & & \\
\hline
\end{tabular}

Notes on Squid:

Lots: I crustaces remains

Notes on Fish:

General Notes:

Crustacea remains with a few fish bones.

PACIFIC OCEANOGRAPHIC BIOLOGICAL SURVEY PROGRAM

SMITHSONIAN OCEANOGRAPHIC SORTING CENTER—SMITHSONIAN INSTITUTION

P. Grisham 8/5/66 
SOSC REF. NO. 229

\begin{tabular}{|c|c|c|c|c|c|c|c|c|c|}
\hline $\begin{array}{l}\text { Lat \& } \\
\text { Long. }\end{array}$ & Date & $\begin{array}{c}\text { Time } \\
\text { Caught }\end{array}$ & Squid & $\begin{array}{l}\text { Squid } \\
\text { Beaks }\end{array}$ & Fish & $\begin{array}{c}\text { Fish } \\
\text { Remains }\end{array}$ & $\begin{array}{l}\text { Parasitic } \\
\text { Animals }\end{array}$ & Crustacea & $\begin{array}{c}\text { Other } \\
\text { Animals }\end{array}$ \\
\hline & $\begin{array}{l}\text { Oct. } \\
20, \\
65\end{array}$ & $\begin{array}{l}\text { Baker } \\
\text { Island }\end{array}$ & & & & - & & & ti \\
\hline
\end{tabular}

Parasitic Animals:

Notes on Squid:

lots: 1 seeds

Notes on Fish:

General Notes:

PACIFIC OCEANOGRAPHIC BIOLOGICAL SURVEY PROGRAM SMITHSONIAN OCEANOGRAPHIC SORTING CENTER_SMITHSONIAN INSTITUTION

S1-USNM-830

P. Grisham 9/16/66

$\underset{7-85}{51 \cdot-48}$ 5

SOSC REF. NO.

POBSP. NO 10

\begin{tabular}{|c|c|c|c|c|c|c|c|c|c|}
\hline $\begin{array}{l}\text { Lat \& } \\
\text { Long. }\end{array}$ & Date & $\begin{array}{l}\text { Time } \\
\text { Caught }\end{array}$ & Squid & $\begin{array}{l}\text { Squid } \\
\text { Beaks }\end{array}$ & Fish & $\begin{array}{c}\text { Fish } \\
\text { Remains }\end{array}$ & $\begin{array}{l}\text { Parasitic } \\
\text { Animals }\end{array}$ & Crustacea & $\begin{array}{c}\text { Other } \\
\text { Animals }\end{array}$ \\
\hline & $\begin{array}{l}\text { Oct. } \\
28, \\
65\end{array}$ & $\begin{array}{l}\text { Xmas } \\
\text { Island }\end{array}$ & & & & - & & & \\
\hline
\end{tabular}

Parasitic Animals:

Lots: 1 digested remains

Notes on Squid:

Notes on Fish:

General Notes:

Digested remains beyond identification.

PACIFIC OCEANOGRAPHIC BIOLOGICAL SURVEY PROGRAM

SMITHSONIAN OCEANOGRAPHIC SORTING CENTER_SMITHSONIAN INSTITUTION 


\begin{tabular}{|c|c|c|c|c|c|c|c|c|c|}
\hline Sta. No. & 5827 & & Species & The & sseus & bergii & & $\begin{array}{ll}\text { No. } & 22 \\
\text { NO. } & 10\end{array}$ & \\
\hline \multirow[t]{2}{*}{$\begin{array}{l}\text { Lat \& } \\
\text { Long. }\end{array}$} & Date & $\begin{array}{l}\text { Time } \\
\text { Caught }\end{array}$ & Squid & $\begin{array}{l}\text { Squid } \\
\text { Beaks }\end{array}$ & Fish & $\begin{array}{c}\text { Fish } \\
\text { Remains }\end{array}$ & $\begin{array}{l}\text { Parasitic } \\
\text { Animals }\end{array}$ & Crustacea & $\begin{array}{c}\text { Other } \\
\text { Animals }\end{array}$ \\
\hline & $\begin{array}{l}\text { Oct. } \\
28, \\
65\end{array}$ & $\begin{array}{l}\text { Xmas } \\
\text { Island }\end{array}$ & & & & + & & & \\
\hline
\end{tabular}

Parasitic Animals:

lots: 1 fish remains

Notes on Squid:

Notes on Fish:

Fish remains with bones.

General Notes:

PACIFIC OCEANOGRAPHIC BIOLOGICAL SURVEY PROGRAM

SMITHSONIAN OCEANOGRAPHIC SORTING CENTER—SMITHSONIAN INSTITUTION

P. Grisham 8/9/66

Sta. No. $5822 \quad$ Species Vini kuhli

SOSC REF. NO. 229

\begin{tabular}{c|c|c|c|c|c|c|c|c|}
\hline $\begin{array}{l}\text { Lat \& } \\
\text { Long. }\end{array}$ & Date & $\begin{array}{l}\text { Time } \\
\text { Caught }\end{array}$ & Squid & $\begin{array}{c}\text { Squid } \\
\text { Beaks }\end{array}$ & Fish & $\begin{array}{c}\text { Fish } \\
\text { Remains }\end{array}$ & $\begin{array}{c}\text { Parasitic } \\
\text { Animals }\end{array}$ & Other \\
\hline & $\begin{array}{l}\text { Oct. } \\
28, \\
65\end{array}$ & $\begin{array}{l}\text { Xmas } \\
\text { Island }\end{array}$ & Animals & & \\
\hline
\end{tabular}

Parasitic Animals:

lots: 1 digested remains

Notes on Squid:

Notes on Fish:

General Notes:

Digested remains beyond identification.

PACIFIC OCEANOGRAPHIC BIOLOGICAL SURVEY PROGRAM

SMITHSONIAN OCEANOGRAPHIC SORTING CENTER—SMITHSONIAN INSTITUTION

P. Grisham $8 / 8 / 66$ 
SOSC REF. NO. 229

\begin{tabular}{l|l|l|l|l|l|l|l|l|l} 
Sta. No. & 5823 & \multicolumn{2}{c}{ Species } & \multicolumn{2}{c}{ Gygis albs } \\
\hline $\begin{array}{l}\text { Lat \& } \\
\text { Long. }\end{array}$ & Date & $\begin{array}{c}\text { Time } \\
\text { Caught }\end{array}$ & Squid & $\begin{array}{l}\text { Squid } \\
\text { Beaks }\end{array}$ & Fish & $\begin{array}{c}\text { Fish } \\
\text { Remains }\end{array}$ & $\begin{array}{c}\text { Parasitic } \\
\text { Animals }\end{array}$ & Crustacea & $\begin{array}{c}\text { Other } \\
\text { Animals }\end{array}$ \\
\hline & $\begin{array}{l}\text { Oct. } \\
28, \\
65\end{array}$ & $\begin{array}{l}\text { Xmas } \\
\text { Island }\end{array}$ & & 1 & & & & Crab (1) & \\
\end{tabular}

Parasitic Animals:

Iots: $3 \mathrm{crab}$

Notes on Squid:

squid beaks

crustacea remains

Notes on Fish:

General Notes:

Crab in fair condition.

PACIFIC OCEANOGRAPHIC BIOLOGICAL SURVEY PROGRAM

SMITHSONIAN OCEANOGRAPHIC SORTING CENTER—SMITHSONIAN INSTITUTION

S1. USNM-\$30

P. Grisham 8/16/66

7.65

7

Sta. No.

Lat \&
Long.

Notes on Squid:

\begin{tabular}{|l|}
\hline Date \\
\hline Oct. \\
28, \\
65
\end{tabular}

5824

Species

Thalasseus bergif

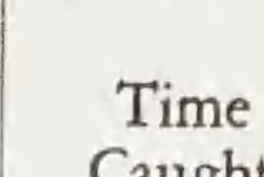

Caught

Xmas

Island

Parasitic Animals:

\section{Notes on Fish:}

General Notes:

Crustacea remains with fish bones.

PACIFIC OCEANOGRAPHIC BIOLOGICAL SURVEY PROGRAM

SMITHSONIAN OCEANOGRAPHIC SORTING CENTER—SMITHSONIAN INSTITUTION

S1-USMM-630
SOSC REF. NO. 229

POBSP. NO. 10

Lots: 2 fish

crustacea remains
Other Animais 


\begin{tabular}{|c|c|c|c|c|c|c|c|c|c|}
\hline Sta. No. & & 5825 & Specie: & The & sseus & berg11 & & $\begin{array}{l}\text { NO. } \\
\text { NO. }\end{array}$ & \\
\hline \multirow[t]{2}{*}{$\begin{array}{l}\text { Lat \& } \\
\text { Long. }\end{array}$} & Date & $\begin{array}{c}\text { Time } \\
\text { Caught }\end{array}$ & Squid & $\begin{array}{l}\text { Squid } \\
\text { Beaks }\end{array}$ & Fish & $\begin{array}{c}\text { Fish } \\
\text { Remains }\end{array}$ & $\begin{array}{l}\text { Parasitic } \\
\text { Animals }\end{array}$ & Crustacea & $\begin{array}{c}\text { Other } \\
\text { Animals }\end{array}$ \\
\hline & $\begin{array}{l}\text { Oct. } \\
28, \\
65\end{array}$ & $\begin{array}{l}X_{\text {mas }} \\
\text { Island }\end{array}$ & & & & - & & & \\
\hline
\end{tabular}

Parasitic Animals:

Iots: 0

Notes on Squid:

Notes on Fish:

General Notes:

The stomach was empty.

PACIFIC OCEANOGRAPHIC BIOLOGICAL SURVEY PROGRAM

SMITHSONIAN OCEANOGRAPHIC SORTING CENTER_SMITHSONIAN INSTITUTION

ร1-USNM-830

$7-68$

\author{
. Grisham 8/1/66
}

3

Sta. No.

5826

Species

Pluvialis dominica

SOSC REF. NO. 229

\begin{tabular}{|c|c|c|c|c|c|c|c|c|c|}
\hline $\begin{array}{l}\text { Lat \& } \\
\text { Long. }\end{array}$ & Date & $\begin{array}{l}\text { Time } \\
\text { Caught }\end{array}$ & Squid & $\begin{array}{l}\text { Squid } \\
\text { Beaks }\end{array}$ & Fish & $\begin{array}{c}\text { Fish } \\
\text { Remains }\end{array}$ & $\begin{array}{l}\text { Parasitic } \\
\text { Animals }\end{array}$ & Crustacea & $\begin{array}{c}\text { Other } \\
\text { Animals }\end{array}$ \\
\hline & $\begin{array}{l}\text { Oct. } \\
28, \\
65\end{array}$ & $\begin{array}{l}\text { Xmas } \\
\text { Island }\end{array}$ & & & & - & & & \\
\hline
\end{tabular}

Parasitic Animals:

Notes on Squid:

1ats: 1 digested remains

Notes on Fish:

General Notes:

Digested remains with rocks and stones.

PACIFIC OCEANOGRAPHIC BIOLOGICAL SURVEY PROGRAM

SMITHSONIAN OCEANOGRAPHIC SORTING CENTER—SMITHSONIAN INSTITUTION 
SOSC REF. NO. 229

\begin{tabular}{|c|c|c|c|c|c|c|c|c|c|}
\hline $\begin{array}{l}\text { Lat \& } \\
\text { Long. }\end{array}$ & Date & $\begin{array}{l}\text { Time } \\
\text { Caught }\end{array}$ & Squid & $\begin{array}{l}\text { Squid } \\
\text { Beaks }\end{array}$ & Fish & $\begin{array}{c}\text { Fish } \\
\text { Remains }\end{array}$ & $\begin{array}{l}\text { Parasitic } \\
\text { Animals }\end{array}$ & Crustacea & $\begin{array}{l}\text { Other } \\
\text { Animals }\end{array}$ \\
\hline & $\begin{array}{l}\text { Oct. } \\
28, \\
65\end{array}$ & $\begin{array}{l}X \text { mas } \\
\text { Island }\end{array}$ & & & & - & & & \\
\hline
\end{tabular}

Parasitic Animals:

lots: I insect remains

Notes on Squid:

Notes on Fish:

General Notes:

PACIFIC OCEANOGRAPHIC BIOLOGICAL SURVEY PROGRAM SMITHSONIAN OCEANOGRAPHIC SORTING CENTER—SMITHSONIAN INSTITUTION

P. Grisham 7/27/66

SI-USNM- -30

$7-65$

SOSC REF. NO. 229

POBSP. NO. 10

Sta. No.

Species

Numenius tahitiensis

\begin{tabular}{|c|c|c|c|c|c|c|c|c|c|}
\hline $\begin{array}{l}\text { Lat \& } \\
\text { Long. }\end{array}$ & Date & $\begin{array}{l}\text { Time } \\
\text { Caught }\end{array}$ & Squid & $\begin{array}{l}\text { Squid } \\
\text { Beaks }\end{array}$ & Fish & $\begin{array}{c}\text { Fish } \\
\text { Remains }\end{array}$ & $\begin{array}{l}\text { Parasitic } \\
\text { Animals }\end{array}$ & Crustacea & $\begin{array}{c}\text { Other } \\
\text { Animals }\end{array}$ \\
\hline & $\begin{array}{l}\text { Oct. } \\
28, \\
65\end{array}$ & $\begin{array}{l}\text { Xmas } \\
\text { Island }\end{array}$ & & & & - & & & \\
\hline
\end{tabular}

Parasitic Animals:

Lots: 2 seeds

Notes on Squid:

insect remains

Notes on Fish:

General Notes:

PACIFIC OCEANOGRAPHIC BIOLOGICAL SURVEY PROGRAM

SMITHSONIAN OCEANOGRAPHIC SORTING CENTER—SMITHSONIAN INSTITUTION

P. Grisham $8 / 2 / 66$ 
SOSC REF. NO. 211

Sta. No. $5829 \quad$ Species Numenius tahitiensis POBSP. NO. 10

\begin{tabular}{c|c|c|c|c|c|c|c|c|}
\hline $\begin{array}{l}\text { Lat \& } \\
\text { Long. }\end{array}$ & Date & $\begin{array}{c}\text { Time } \\
\text { Caught }\end{array}$ & Squid & $\begin{array}{c}\text { Squid } \\
\text { Beaks }\end{array}$ & Fish & $\begin{array}{c}\text { Fish } \\
\text { Remains }\end{array}$ & $\begin{array}{c}\text { Parasitic } \\
\text { Animals }\end{array}$ & $\begin{array}{c}\text { Other } \\
\text { Animals }\end{array}$ \\
\hline & $\begin{array}{l}\text { Oct. } \\
28, \\
65\end{array}$ & $\begin{array}{l}\text { Xmas } \\
\text { Island }\end{array}$ & & & & & & \\
\hline
\end{tabular}

Parasitic Animals:

Lots: 1 seeds

Notes on Squid:

Notes on Fish:

General Notes:

Seeds with a few crustacea remains.

PACIFIC OCEANOGRAPHIC BIOLOGICAL SURVEY PROGRAM SMITHSONIAN OCEANOGRAPHIC SORTING CENTER—SMITHSONIAN INSTITUTION

\section{P. Grisham 8/8/66}

\begin{tabular}{|c|c|c|c|c|c|c|c|c|c|}
\hline Sta. No. & 5831 & & Specie & Sul & dacty & tra & & $\begin{array}{l}\text { No. } \\
\text { NO. }\end{array}$ & \\
\hline \multirow[t]{2}{*}{$\begin{array}{l}\text { Lat \& } \\
\text { Long. }\end{array}$} & Date & $\begin{array}{c}\text { Time } \\
\text { Caught }\end{array}$ & Squid & $\begin{array}{l}\text { Squid } \\
\text { Beaks }\end{array}$ & Fish & $\begin{array}{c}\text { Fish } \\
\text { Remains }\end{array}$ & $\begin{array}{l}\text { Parasitic } \\
\text { Animals }\end{array}$ & Crustacea & $\begin{array}{c}\text { Other } \\
\text { Animals }\end{array}$ \\
\hline & $\begin{array}{l}\text { Oct. } \\
29, \\
65\end{array}$ & $\begin{array}{l}\text { Xmas } \\
\text { Island }\end{array}$ & & 23 & & - & & & \\
\hline
\end{tabular}

Notes on Squid:

lots: 2 squid beaks digested remains eyebalis (7)

Notes on Fish:

General Notes:

No stomach, only stomach contents.

PACIFIC OCEANOGRAPHIC BIOLOGICAL SURVEY PROGRAM SMITHSONIAN OCEANOGRAPHIC SORTING CENTER — SMITHSONIAN INSTITUTION 


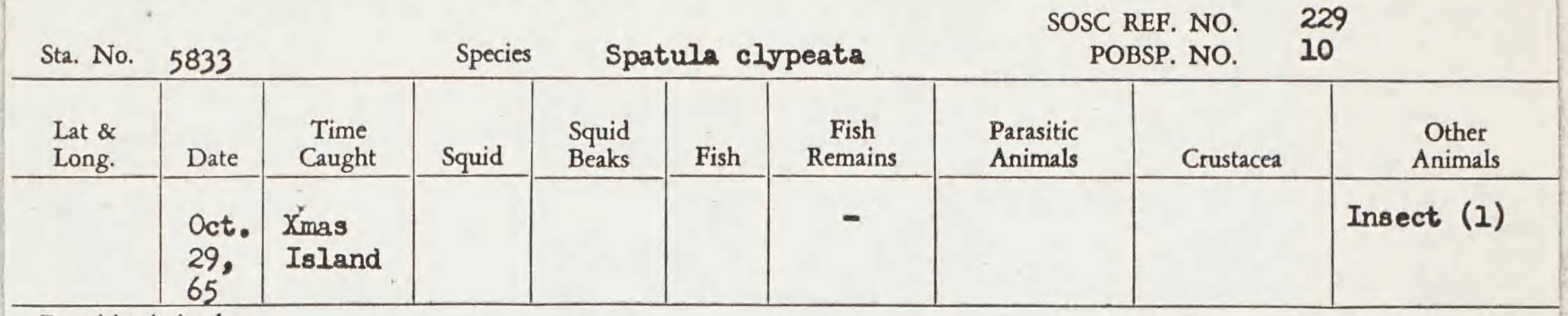

Parasitic Animals:

Notes on Squid:

1ots: 2 insect remains Insect

Notes on Fish:

General Notes:

Insect in good condition.

PACIFIC OCEANOGRAPHIC BIOLOGICAL SURVEY PROGRAM

SMITHSONIAN OCEANOGRAPHIC SORTING CENTER_-SMITHSONIAN INSTITUTION

SI.USNM-830

\begin{tabular}{|c|c|c|c|c|c|c|}
\hline Sta. No. & \multicolumn{2}{|c|}{5863} & Specie & \multicolumn{2}{|c|}{ Pterodroma } & \\
\hline $\begin{array}{l}\text { Lat \& } \\
\text { Long. }\end{array}$ & Date & $\begin{array}{l}\text { Time } \\
\text { Caught }\end{array}$ & Squid & $\begin{array}{l}\text { Squid } \\
\text { Beaks }\end{array}$ & Fish & $\begin{array}{c}\text { Fish } \\
\text { Remains }\end{array}$ \\
\hline & $\begin{array}{l}\text { Oct. } \\
30 \\
65\end{array}$ & $\begin{array}{l}\text { Xmas } \\
\text { Island }\end{array}$ & 3 & & & - \\
\hline
\end{tabular}

SOSC REF. NO. 229

Parasitic Animals:

lots: 3 ejeballs (4) squid beaks

Notes on Squid:

Heads of two digested. squid remains

Notes on Fish:

General Notes:

PACIFIC OCEANOGRAPHIC BIOLOGICAL SURVEY PROGRAM SMITHSONIAN OCEANOGRAPHIC SORTING CENTER—SMITHSONIAN INSTITUTION

P. Grisham $8 / 1 / 66$ 
SOSC REF. NO. 229

\begin{tabular}{|c|c|c|c|c|c|c|c|c|c|}
\hline $\begin{array}{l}\text { Lat \& } \\
\text { Long. }\end{array}$ & Date & $\begin{array}{l}\text { Time } \\
\text { Caught }\end{array}$ & Squid & $\begin{array}{l}\text { Squid } \\
\text { Beaks }\end{array}$ & Fish & $\begin{array}{c}\text { Fish } \\
\text { Remains }\end{array}$ & $\begin{array}{l}\text { Parasitic } \\
\text { Animals }\end{array}$ & Crustacea & $\begin{array}{c}\text { Other } \\
\text { Animals }\end{array}$ \\
\hline & $\begin{array}{l}\text { Oct. } \\
30, \\
65\end{array}$ & $\begin{array}{l}\text { Xmas } \\
I \text { Is and }\end{array}$ & & 6 & & - & & & \\
\hline
\end{tabular}

Parasitic Animals:

Notes on Squid:

Iots: 2 squid beaks digested remains

Notes on Fish:

General Notes:

Digested remains beyond identification.

PACIFIC OCEANOGRAPHIC BIOLOGICAL SURVEY PROGRAM SMITHSONIAN OCEANOGRAPHIC SORTING CENTER_SMITHSONIAN INSTITUTION

S1.USNM-830 P. Grisham 8/9/66

7-65

\begin{tabular}{|c|c|}
\hline $\begin{array}{l}\text { Lat \& } \\
\text { Long. }\end{array}$ & Date \\
\hline & $\begin{array}{l}\text { Oct. } \\
30, \\
65\end{array}$ \\
\hline
\end{tabular}

Parasitic Animals:

Notes on Squid:

lots: 2 squid beaks digested remains

Notes on Fish:

General Notes:

PACIFIC OCEANOGRAPHIC BIOLOGICAL SURVEY PROGRAM SMITHSONIAN OCEANOGRAPHIC SORTING CENTER—SMITHSONIAN INSTITUTION

P. Grisham 8/15/66 


\begin{tabular}{|c|c|c|c|c|c|c|c|c|c|}
\hline Sta. No. & 5867 & & Speci & & suls & & & $\begin{array}{l}\text { No. } \\
\text { No. }\end{array}$ & \\
\hline \multirow[t]{2}{*}{$\begin{array}{l}\text { Lat \& } \\
\text { Long. }\end{array}$} & Date & $\begin{array}{c}\text { Time } \\
\text { Caught }\end{array}$ & Squid & $\begin{array}{l}\text { Squid } \\
\text { Beaks }\end{array}$ & Fish & $\begin{array}{c}\text { Fish } \\
\text { Remains }\end{array}$ & $\begin{array}{l}\text { Parasitic } \\
\text { Animals }\end{array}$ & Crustacea & $\begin{array}{c}\text { Other } \\
\text { Animals }\end{array}$ \\
\hline & $\begin{array}{l}\text { Oct. } \\
30, \\
65\end{array}$ & $\begin{array}{l}\text { Xmas } \\
\text { Island }\end{array}$ & & & & - & & & \\
\hline
\end{tabular}

Parasitic Animals:

lats: 2 eyebs]ls (2)

Notes on Squid:

Squid remalns with half of aquid. squid remains

Notes on Fish:

General Notes:

PACIFIC OCEANOGRAPHIC BIOLOGICAL SURVEY PROGRAM

SMITHSONIAN OCEANOGRAPHIC SORTING CENTER—SMITHSONIAN INSTITUTION

S1-USNM- 30

P. Grisham 8/1/66

SOSC REF. NO. 229

Sta. No. 20282,30

Species Totanus flavipes

POBSP. NO. 10

\begin{tabular}{|c|c|c|c|c|c|c|c|c|c|}
\hline $\begin{array}{l}\text { Lat \& } \\
\text { Long. }\end{array}$ & Date & $\begin{array}{l}\text { Time } \\
\text { Caught }\end{array}$ & Squid & $\begin{array}{l}\text { Squid } \\
\text { Beaks }\end{array}$ & Fish & $\begin{array}{c}\text { Fish } \\
\text { Remains }\end{array}$ & $\begin{array}{l}\text { Parasitic } \\
\text { Animais }\end{array}$ & Crustacea & $\begin{array}{c}\text { Other } \\
\text { Animals }\end{array}$ \\
\hline & $\begin{array}{l}\text { Sept. } \\
2, \\
64\end{array}$ & $\begin{array}{l}\text { Green } \\
\text { Island }\end{array}$ & & & & $\infty$ & & & \\
\hline
\end{tabular}

Parasitic Animals:

lots: I digested remains

Notes on Squid:

Notes on Fish:

General Notes:

Digested remains beyond identification.

PACIFIC OCEANOGRAPHIC BIOLOGICAL SURVEY PROGRAM

SMITHSONIAN OCEANOGRAPHIC SORTING CENTER_-SMITHSONIAN INSTITUTION 


\begin{tabular}{|c|c|c|c|c|c|c|c|c|c|}
\hline Sta. No. & 202 & & Speci & Sula & & & & $\begin{array}{l}\text { NO. } \\
\text { NO. }\end{array}$ & \\
\hline \multirow[t]{2}{*}{$\begin{array}{l}\text { Lat \& } \\
\text { Long. }\end{array}$} & Date & $\begin{array}{l}\text { Time } \\
\text { Caught }\end{array}$ & Squid & $\begin{array}{l}\text { Squid } \\
\text { Beaks }\end{array}$ & Fish & $\begin{array}{c}\text { Fish } \\
\text { Remains }\end{array}$ & $\begin{array}{l}\text { Parasitic } \\
\text { Animals }\end{array}$ & Crustacea & $\begin{array}{c}\text { Other } \\
\text { Animals }\end{array}$ \\
\hline & $\begin{array}{l}\text { Sept. } \\
11 \text {, } \\
64\end{array}$ & $\begin{array}{l}\text { Groen } \\
\text { Island }\end{array}$ & & & & 1 & & & \\
\hline
\end{tabular}

Parasitic Animals:

lots: 1 digested remains

Notes on Squid:

Notes on Fish:

General Notes:

Digested remains beyond identification.

PACIFIC OCEANOGRAPHIC BIOLOGICAL SURVEY PROGRAM

SMITHSONIAN OCEANOGRAPHIC SORTING CENTER_SMITHSONIAN INSTITUTION

P. Grisham 9/14/66

S1.USNM- -30

T1-6s

$$
5
$$

\begin{tabular}{|c|c|c|c|c|c|c|c|c|c|}
\hline Sta. No. & 2044 & & Speci & Gyt & alb & & & $\begin{array}{l}\text { NO. } \\
\text { No. }\end{array}$ & \\
\hline \multirow[t]{2}{*}{$\begin{array}{l}\text { Lat \& } \\
\text { Long. }\end{array}$} & Date & $\begin{array}{l}\text { Time } \\
\text { Caught }\end{array}$ & Squid & $\begin{array}{l}\text { Squid } \\
\text { Beaks }\end{array}$ & Fish & $\begin{array}{c}\text { Fish } \\
\text { Remains }\end{array}$ & $\begin{array}{l}\text { Parasitic } \\
\text { Animals }\end{array}$ & Crustacea & $\begin{array}{l}\text { Other } \\
\text { Animals }\end{array}$ \\
\hline & $\begin{array}{l}\text { Feb. } \\
25, \\
65\end{array}$ & $\begin{array}{l}\text { Green } \\
\text { Island }\end{array}$ & & & & - & & & \\
\hline
\end{tabular}

Parasitic Animals:

Lots: 0

Notes on Squid:

Notes on Fish:

General Notes:

THE stomach was empty.

PACIFIC OCEANOGRAPHIC BIOLOGICAL SURVEY PROGRAM

SMITHSONIAN OCEANOGRAPHIC SORTING CENTER_SMITHSONIAN INSTITUTION 
SOSC REF. NO. 229

\begin{tabular}{|c|c|c|c|c|c|c|c|c|c|}
\hline $\begin{array}{l}\text { Lat \& } \\
\text { Long. }\end{array}$ & Date & $\begin{array}{c}\text { Time } \\
\text { Caught }\end{array}$ & Squid & $\begin{array}{l}\text { Squid } \\
\text { Beaks }\end{array}$ & Fish & $\begin{array}{c}\text { Fish } \\
\text { Remains }\end{array}$ & $\begin{array}{l}\text { Parasitic } \\
\text { Animals }\end{array}$ & Crustacea & $\begin{array}{c}\text { Other } \\
\text { Animals }\end{array}$ \\
\hline & $\begin{array}{l}\text { May } \\
25, \\
65\end{array}$ & $\begin{array}{l}\text { Green } \\
\text { Island }\end{array}$ & & 8 & & - & $\begin{array}{c}\text { Nematodes } \\
(4)\end{array}$ & & \\
\hline
\end{tabular}

Parasitic Animals:

Notes on Squid:

lots: 3 squid beaks

digested remains nematodes

Notes on Fish:

General Notes:

PACIFIC OCEANOGRAPHIC BIOLOGICAL SURVEY PROGRAM SMITHSONIAN OCEANOGRAPHIC SORTING CENTER — SMITHSONIAN INSTITUTION

81-บ8nm-aso

T-08

$$
\text { P. Grisham 8/1/66 }
$$

\section{5}

SOSC REF. NO.

229

Sta. No.

20478

Species Sula sula

POBSP. NO.

10

\begin{tabular}{c|c|c|c|c|c|c|c|c}
\hline $\begin{array}{l}\text { Lat \& } \\
\text { Long. }\end{array}$ & Date & $\begin{array}{c}\text { Time } \\
\text { Caught }\end{array}$ & Squid & $\begin{array}{c}\text { Squid } \\
\text { Beaks }\end{array}$ & Fish & $\begin{array}{c}\text { Fish } \\
\text { Remains }\end{array}$ & $\begin{array}{c}\text { Parasitic } \\
\text { Animals }\end{array}$ & $\begin{array}{c}\text { Other } \\
\text { Animals }\end{array}$ \\
\hline & $\begin{array}{l}\text { May } \\
27, \\
65\end{array}$ & $\begin{array}{l}\text { Green } \\
\text { Island }\end{array}$ & & & & & & \\
\hline
\end{tabular}

Parasitic Animals:

Notes on Squid:

Lots: I Ish remains

Notes on Fish:

Fish remains with bones and half of a fish.

General Notes:

PACIFIC OCEANOGRAPHIC BIOLOGICAL SURVEY PROGRAM SMITHSONIAN OCEANOGRAPHIC SORTING CENTER—SMITHSONIAN INSTITUTION 
SOSC REF. NO. 229

\begin{tabular}{|c|c|c|c|c|c|c|c|c|c|}
\hline Sta. No. & 2048 & & Speci & Fre & a $\mathrm{m}^{-1}$ & & & NO. 10 & \\
\hline \multirow[t]{2}{*}{$\begin{array}{l}\text { Lat \& } \\
\text { Long. }\end{array}$} & Date & $\begin{array}{c}\text { Time } \\
\text { Caught }\end{array}$ & Squid & $\begin{array}{l}\text { Squid } \\
\text { Beaks }\end{array}$ & Fish & $\begin{array}{c}\text { Fish } \\
\text { Remains }\end{array}$ & $\begin{array}{l}\text { Parasitic } \\
\text { Animals }\end{array}$ & Crustacea & $\begin{array}{l}\text { Other } \\
\text { Animals }\end{array}$ \\
\hline & $\begin{array}{l}\text { June } \\
3, \\
65\end{array}$ & $\begin{array}{l}\text { Green } \\
\text { Island }\end{array}$ & & 3 & & + & & & \\
\hline
\end{tabular}

Parasitic Animals:

Notes on Squid:

Lots: 2 fish remains squid beaks

Notes on Fish:

Fish remains with bones.

General Notes:

PACIFIC OCEANOGRAPHIC BIOLOGICAL SURVEY PROGRAM

SMITHSONIAN OCEANOGRAPHIC SORTING CENTER — SMITHSONIAN INSTITUTION

P. Grisham 8/4/66

$\underset{7-65}{\text { S1-USNm-830 }}$

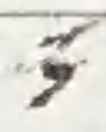

\begin{tabular}{|c|c|c|c|c|c|c|}
\hline Sta. No. & \multicolumn{2}{|c|}{20482} & Speci & \multicolumn{3}{|c|}{ Fregata minor } \\
\hline $\begin{array}{l}\text { Lat \& } \\
\text { Long. }\end{array}$ & Date & $\begin{array}{c}\text { Time } \\
\text { Caught }\end{array}$ & Squid & $\begin{array}{l}\text { Squid } \\
\text { Beaks }\end{array}$ & Fish & $\begin{array}{c}\text { Fish } \\
\text { Remains }\end{array}$ \\
\hline & $\begin{array}{l}\text { June } \\
3, \\
65\end{array}$ & $\begin{array}{l}\text { Green } \\
\text { Island }\end{array}$ & & 4 & 1 & + \\
\hline
\end{tabular}

SOSC REF. NO. 229

Parasitic Animals:

Notes on Squid:

lats: 5 squid beaks eyeballs (5) nematodes(1) fish

Notes on Fish: 1. Head of fish digested. fish remains

2. Fish remains with bones.

\begin{tabular}{|c|c|c}
$\begin{array}{c}\text { Parasitic } \\
\text { Animals }\end{array}$ & Crustacea & $\begin{array}{c}\text { Other } \\
\text { Animals }\end{array}$ \\
\hline $\begin{array}{c}\text { Nematodes } \\
\text { (I) }\end{array}$ & &
\end{tabular}

General Notes:

PACIFIC OCEANOGRAPHIC BIOLOGICAL SURVEY PROGRAM

SMITHSONIAN OCEANOGRAPHIC SORTING CENTER—SMITHSONIAN INSTITUTION

P. Grisham $8 / 17 / 66$ 


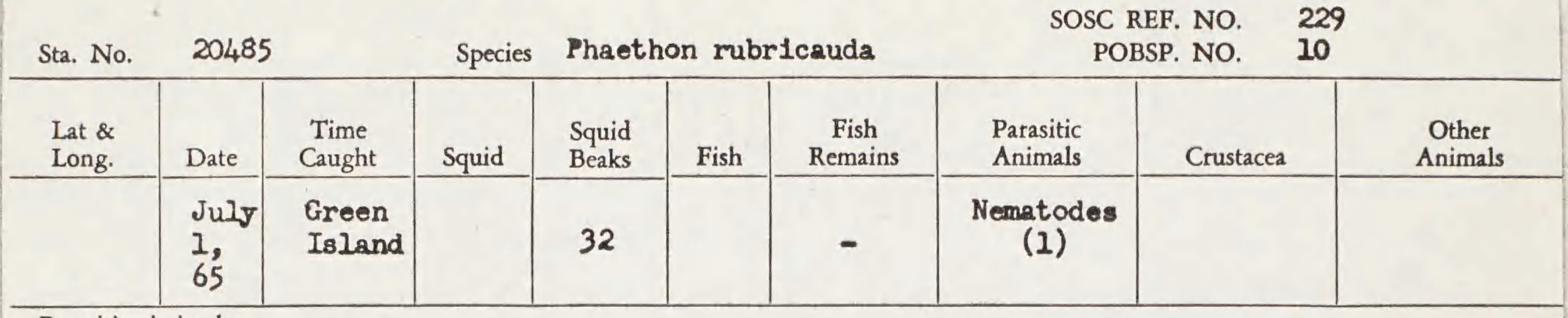

Parasitic Animals:

Notes on Squid:

1ots: 3 squid beaks nematodes digested remains

Notes on Fish:

General Notes:

PACIFIC OCEANOGRAPHIC BIOLOGICAL SURVEY PROGRAM SMITHSONIAN OCEANOGRAPHIC SORTING CENTER—SMITHSONIAN INSTITUTION P. Grisham 8/2/66
SMITHSONIAN
P. Grisham 3

SOSC REF. NO.

229

POBSP. NO. 10

\begin{tabular}{c|l} 
Sta. No. & \\
\hline Lat \& & \\
Long. & Date \\
\hline & $\begin{array}{l}\text { Oct. } \\
15, \\
64\end{array}$ \\
& \\
\hline
\end{tabular}

Parasitic Animals:

Lots: 1 crustacea remains

Notes on Squid:

Notes on Fish:

General Notes:

Crustacea remains with claws and appendages.

PACIFIC OCEANOGRAPHIC BIOLOGICAL SURVEY PROGRAM

SMITHSONIAN OCEANOGRAPHIC SORTING CENTER_SMITHSONIAN INSTITUTION 
Parasitic Animals:

lota: 1 crustacea remains

Notes on Squid:

Notes on Fish:

General Notes:

PACIFIC OCEANOGRAPHIC BIOLOGICAL SURVEY PROGRAM

SMITHSONIAN OCEANOGRAPHIC SORTING CENTER—SMITHSONIAN INSTITUTION

P. Grisham 9/6/66

Sta. No.

\begin{tabular}{|l|l|l|}
\multicolumn{2}{c|}{15008} & Species \\
Date & $\begin{array}{c}\text { Time } \\
\text { Caught }\end{array}$ & Squid \\
\hline $\begin{array}{l}\text { Aug. } \\
18, \\
65\end{array}$ & Hawa11 & \\
\hline
\end{tabular}

Parasitic Animals:

\section{Notes on Squid:}

Notes on Fish:

General Notes:

Digested remains byyond identification.

lots: 1 digested cemains

\begin{tabular}{|c|c} 
Crustacea & $\begin{array}{c}\text { Other } \\
\text { Animals }\end{array}$ \\
\hline &
\end{tabular}

SOSC REF. NO. 229

POBSP. NO. 10 
Wish remains indicated

SOSC RET: NTO. $\frac{1}{229}$

Dy + or - sign

POBSP NO. 10

\begin{tabular}{|c|c|c|c|c|c|c|c|c|}
\hline Sta. No. & Species & Squia & $\begin{array}{l}\text { Squid } \\
\text { Beaks } \\
\end{array}$ & Fish & $\begin{array}{l}\text { Fish } \\
\text { Rem.* }\end{array}$ & $\begin{array}{l}\text { Parasitic } \\
\text { Animais } \\
\end{array}$ & Crustacea & $\begin{array}{c}\text { Other } \\
\text { Animals }\end{array}$ \\
\hline 0951 & $\begin{array}{l}\text { Sterna } \\
\text { fuscata }\end{array}$ & & 9 & & - & & & \\
\hline 0952 & $\begin{array}{l}\text { Sula } \\
\text { dactylatra }\end{array}$ & & 2 & & - & & & \\
\hline 0953 & $\begin{array}{l}\text { Puffinus } \\
\text { pacificus }\end{array}$ & & 124 & & - & & & \\
\hline 0954 & $\begin{array}{l}\text { Fuffinus } \\
\text { pacificus }\end{array}$ & 1 & 9 & & - & & & \\
\hline 0955 & $\begin{array}{l}\text { Sula } \\
\text { dactylatra }\end{array}$ & & 7 & 1 & + & & & 2 \\
\hline 0956 & $\begin{array}{l}\text { Sula } \\
\text { dactylatra }\end{array}$ & & 2 & 2 & + & 1 & & \\
\hline 0957 & $\begin{array}{l}\text { Sula } \\
\text { dactylatra }\end{array}$ & & 18 & & + & & & \\
\hline 0958 & $\begin{array}{l}\text { Sula } \\
\text { dactylatra }\end{array}$ & & & & - & 15 & & \\
\hline 0959 & Sula & & 5 & & - & & & \\
\hline 0962 & $\begin{array}{l}\text { Sula } \\
\text { dactylatra }\end{array}$ & & 28 & & + & & & \\
\hline 0962 & $\begin{array}{l}\text { Sula } \\
\text { dactylatra }\end{array}$ & & 7 & 2 & + & & & 6 \\
\hline 0963 & $\begin{array}{l}\text { Sterna } \\
\text { fuscata }\end{array}$ & & & & + & & & \\
\hline 0964 & $\begin{array}{l}\text { Phaethon } \\
\text { rubricauda }\end{array}$ & & & 3 & + & & & 1 \\
\hline 0965 & $\begin{array}{l}\text { Stema } \\
\text { fuscata }\end{array}$ & & & & - & & & \\
\hline 0966 & $\begin{array}{l}\text { Puffinus } \\
\text { griseus }\end{array}$ & 2 & & 2 & + & & & 2 \\
\hline 0967 & $\begin{array}{l}\text { Sula } \\
\text { sula }\end{array}$ & & 121 & & + & & & 6 \\
\hline 0968 & $\begin{array}{l}\text { Sula } \\
\text { sula }\end{array}$ & 2 & 100 & & + & & & 16 \\
\hline 0969 & $\begin{array}{l}\text { Sula } \\
\text { dactylatra }\end{array}$ & & & 3 & + & & & \\
\hline
\end{tabular}


Wish remains indicated

by $\div$ or - sign p. 2 of 15

SOSC REF. NO. 229 POBSP NO. 10

\begin{tabular}{|c|c|c|c|c|c|c|c|c|}
\hline Sta. No. & Spoci: & Squid & $\begin{array}{l}\text { Squia } \\
\text { Beaks } \\
\end{array}$ & Fish & $\begin{array}{l}\text { Fish } \\
\text { Rem.* }\end{array}$ & $\begin{array}{l}\text { Parasitic } \\
\text { Animals }\end{array}$ & Crustacea & $\begin{array}{c}\text { Other } \\
\text { Animels } \\
\end{array}$ \\
\hline 0970 & $\begin{array}{l}\text { Stenwa } \\
\text { fuscata }\end{array}$ & & 2 & & - & & & \\
\hline 0971 & $\begin{array}{l}\text { Sterna } \\
\text { fuscata }\end{array}$ & (Stome & ch empty & & - & & & \\
\hline 0972 & $\begin{array}{l}\text { Sterna } \\
\text { iuscata }\end{array}$ & (Stor) & ach empt & y) & - & & & \\
\hline 0973 & $\begin{array}{l}\text { Sterna } \\
\text { fuscata }\end{array}$ & (Stor) & ach empt & y) & - & & & \\
\hline 097: & $\begin{array}{l}\text { Sterna } \\
\text { Fuscata } \\
\end{array}$ & & 1 & & - & & & \\
\hline 0975 & $\begin{array}{l}\text { terna } \\
\text { fuscata }\end{array}$ & & 2 & & + & & & \\
\hline 0976 & $\begin{array}{l}\text { Sula } \\
\text { sula }\end{array}$ & & & 3 & + & & & \\
\hline 0977 & $\begin{array}{l}\text { Sterna } \\
\text { fuscata }\end{array}$ & & & & + & & & \\
\hline 0978 & $\begin{array}{l}\text { Puffinus } \\
\text { pacificus }\end{array}$ & I & 29 & 1 & - & 1 & & \\
\hline 0979 & $\begin{array}{l}\text { Sterna } \\
\text { fuscata }\end{array}$ & & & & + & & & \\
\hline 0980 & $\begin{array}{l}\text { Phaethon } \\
\text { lepturus }\end{array}$ & & 2 & & + & & & \\
\hline 0981 & $\begin{array}{l}\text { Sterna } \\
\text { fuscata }\end{array}$ & 3 & & I & + & & & \\
\hline 0982 & $\begin{array}{l}\text { Stema } \\
\text { fuscata }\end{array}$ & 2 & & & - & & & \\
\hline 0983 & $\begin{array}{l}\text { Sterna } \\
\text { Puscata }\end{array}$ & 2 & & & + & & & \\
\hline 0984 & $\begin{array}{l}\text { Sterna } \\
\text { fuscata }\end{array}$ & & & & + & & & 1 \\
\hline 0985 & $\begin{array}{l}\text { Sterna } \\
\text { fuscata }\end{array}$ & & & & + & & & \\
\hline 0986 & $\begin{array}{l}\text { Sterna } \\
\text { fuscata }\end{array}$ & & & & - & & & \\
\hline 0987 & $\begin{array}{l}\text { Phaethon } \\
\text { lepturus }\end{array}$ & & 25 & & - & & & 3 \\
\hline
\end{tabular}


Fish remains indicated

by + or - sign p. 3 of 15

SOSC RIIF. NO. 229

POBSP NO. 10

\begin{tabular}{|c|c|c|c|c|c|c|c|c|}
\hline Sta. No. & Species & Squid & $\begin{array}{l}\text { Squia } \\
\text { Beaks } \\
\end{array}$ & Fish & $\begin{array}{l}\text { Fish } \\
\text { Rem.* }\end{array}$ & $\begin{array}{l}\text { Parasitic } \\
\text { Animals }\end{array}$ & Crustacea & $\begin{array}{c}\text { Other } \\
\text { Animels }\end{array}$ \\
\hline 0988 & $\begin{array}{l}\text { Phaethon } \\
\text { rubricauda }\end{array}$ & & 19 & & - & & & 5 \\
\hline 0989 & $\begin{array}{l}\text { Sula } \\
\text { dactylatra }\end{array}$ & & 2 & 2 & + & & & \\
\hline 0990 & $\begin{array}{l}\text { Phaethon } \\
\text { rubricauda }\end{array}$ & & 4 & & + & & & \\
\hline 0991 & $\begin{array}{c}\text { seanodroma } \\
\text { Leucorhoa }\end{array}$ & & & & - & & & \\
\hline 0992 & $\begin{array}{l}\text { Sula } \\
\text { dactylatra }\end{array}$ & & 12 & & + & 1 & & 3 \\
\hline 0993 & $\begin{array}{l}\text { Sterna } \\
\text { fuscata }\end{array}$ & & & & - & & & \\
\hline 0994 & $\begin{array}{l}\text { Sterna } \\
\text { fuscata }\end{array}$ & 1 & & & + & & & \\
\hline 0995 & $\begin{array}{l}\text { Sterna } \\
\text { fuscata }\end{array}$ & 1 & & & + & & & \\
\hline 0996 & $\begin{array}{l}\text { Sterna } \\
\text { fuscata }\end{array}$ & & & 3 & + & & & \\
\hline 0997 & $\begin{array}{l}\text { Oceanodroma } \\
\text { leucorhos }\end{array}$ & & 1 & & - & & & 4 \\
\hline 0998 & $\begin{array}{l}\text { Sterna } \\
\text { fuscata }\end{array}$ & 1 & 1 & & + & & & \\
\hline 0999 & $\begin{array}{l}\text { Sterna } \\
\text { fuscata }\end{array}$ & & & & + & & & \\
\hline 1000 & $\begin{array}{l}\text { Sterna } \\
\text { fuscata }\end{array}$ & 1 & 1 & 1 & - & & & \\
\hline 1001 & $\begin{array}{l}\text { Sterna } \\
\text { fuscata }\end{array}$ & 1 & 4 & & + & & & 2 \\
\hline 1002 & $\begin{array}{l}\text { Sterna } \\
\text { fuscata }\end{array}$ & & 3 & & + & & & 2 \\
\hline 1003 & $\begin{array}{l}\text { Sterna } \\
\text { fuscata }\end{array}$ & 1 & 3 & & + & & & 1 \\
\hline 1004 & $\begin{array}{l}\text { Soma } \\
\text { fuscata }\end{array}$ & & & & + & & & \\
\hline 1005 & $\begin{array}{l}\text { Sterna } \\
\text { fuscata. }\end{array}$ & & 7 & & + & & & \\
\hline
\end{tabular}


- Wish remains indicated

by + or - sign

p. 4 of 15

SOSC RISF. NO. 229

POBSP NO. 10

\begin{tabular}{|c|c|c|c|c|c|c|c|c|}
\hline Sta. No. & Species & Squid & $\begin{array}{l}\text { Squid } \\
\text { Bealks } \\
\end{array}$ & Fish & $\begin{array}{l}\text { Fish } \\
\text { Rem.* }\end{array}$ & $\begin{array}{l}\text { Parasitic } \\
\text { Animals } \\
\end{array}$ & Cmistacea & $\begin{array}{c}\text { Other } \\
\text { Animals }\end{array}$ \\
\hline 1006 & $\begin{array}{l}\text { Sterna } \\
\text { fuscata }\end{array}$ & & & & - & & & 1 \\
\hline 1007 & $\begin{array}{l}\text { Pterodroma } \\
\text { hypoleuca }\end{array}$ & & & & - & & & \\
\hline 1009 & $\begin{array}{l}\text { Sterna } \\
\text { fuscata }\end{array}$ & (Sto & frach emp & $y)$ & - & & & \\
\hline 1010 & $\begin{array}{l}\text { Sterna } \\
\text { fuscata }\end{array}$ & (Sto & hach emp & ty) & - & & & \\
\hline 1011 & $\begin{array}{l}\text { Puffinus } \\
\text { carneipes }\end{array}$ & & 30 & & - & & & 4 \\
\hline 1012 & $\begin{array}{l}\text { Sterna } \\
\text { fuscata }\end{array}$ & & & & + & & & \\
\hline 1013 & $\begin{array}{l}\text { Sterna } \\
\text { fuscata }\end{array}$ & & 1 & 1 & + & 1 & & \\
\hline 1014 & $\begin{array}{l}\text { Sterna } \\
\text { fuscata }\end{array}$ & & & & + & & & \\
\hline 1015 & $\begin{array}{l}\text { Sterna } \\
\text { fuscata }\end{array}$ & 3 & & & + & & & \\
\hline 1026 & $\begin{array}{l}\text { Sterna } \\
\text { fuscata }\end{array}$ & 2 & 3 & & + & & & \\
\hline 1017 & $\begin{array}{l}\text { Sterna } \\
\text { fuscata }\end{array}$ & 1 & 1 & & + & & & \\
\hline 1018 & $\begin{array}{l}\text { Sterna } \\
\text { fuscata }\end{array}$ & & & & + & & & \\
\hline 1019 & $\begin{array}{l}\text { Sterna } \\
\text { fuscata }\end{array}$ & $i$ & 2 & & + & & & \\
\hline 1020 & $\begin{array}{l}\text { Sterna } \\
\text { fuscata }\end{array}$ & 1 & & & + & & & \\
\hline 1021 & $\begin{array}{l}\text { Sterna } \\
\text { fuscata }\end{array}$ & 1 & & & - & & & \\
\hline 2022 & $\begin{array}{l}\text { Sterna } \\
\text { fuscata. }\end{array}$ & 2 & & & + & & & \\
\hline 1023 & $\begin{array}{l}\text { Stema } \\
\text { fuscata }\end{array}$ & 1 & & & - & & & \\
\hline 1024 & $\begin{array}{l}\text { Sterna } \\
\text { fuscata } \\
\end{array}$ & & & & - & & & \\
\hline & & & & & & & & \\
\hline
\end{tabular}


*ish remains indicated

by + or - sign p. 5 of 15

SOSC RIF. NO. 229

POBSP NO. 10

\begin{tabular}{|c|c|c|c|c|c|c|c|c|}
\hline Sta. No. & Species & Squid & $\begin{array}{l}\text { Squià } \\
\text { Beaks } \\
\end{array}$ & Fish & $\begin{array}{l}\text { Fish } \\
\text { Rem.* }\end{array}$ & $\begin{array}{l}\text { Parasitic } \\
\text { Animals }\end{array}$ & Crustacea & $\begin{array}{c}\text { Other } \\
\text { Animals }\end{array}$ \\
\hline 2025 & $\begin{array}{l}\text { Sterna } \\
\text { fuscata }\end{array}$ & & & 1 & + & & & \\
\hline 1026 & $\begin{array}{l}\text { Puffinus } \\
\text { pacificus }\end{array}$ & & 4 & & - & 1 & & 3 \\
\hline 1027 & $\begin{array}{l}\text { Puffinus } \\
\text { pacificus }\end{array}$ & & 7 & & - & & & 1 \\
\hline 1028 & $\begin{array}{l}\text { Sterna } \\
\text { fuscata }\end{array}$ & & & & + & & & \\
\hline 1029 & $\begin{array}{l}\text { Sula } \\
\text { dactylatra }\end{array}$ & & & 3 & + & & & I \\
\hline 1030 & $\begin{array}{l}\text { Sterna } \\
\text { fuscata }\end{array}$ & 2 & 1 & 16 & + & & & \\
\hline 2031 & $\begin{array}{l}\text { Sterna } \\
\text { fuscata }\end{array}$ & & & & + & & & \\
\hline 1032 & $\begin{array}{l}\text { Puffinus } \\
\text { pacificus }\end{array}$ & 2 & 5 & & + & & & \\
\hline 1033 & $\begin{array}{l}\text { Sterna } \\
\text { fuscata }\end{array}$ & & & 1 & + & & & \\
\hline 1034 & $\begin{array}{l}\text { Puffinus } \\
\text { pacificus }\end{array}$ & 1 & 4 & & - & 1 & ': & 2 \\
\hline 1035 & $\begin{array}{l}\text { Fregata } \\
\text { minor }\end{array}$ & & & & + & & & \\
\hline 1036 & $\begin{array}{l}\text { Puffinus } \\
\text { pacificus }\end{array}$ & & 71 & & - & I & & \\
\hline 1037 & $\begin{array}{l}\text { Sula } \\
\text { dactylatra }\end{array}$ & & & & + & & & \\
\hline 1038 & $\begin{array}{l}\text { Sterna } \\
\text { fuscata }\end{array}$ & & & & + & & & \\
\hline 1039 & $\begin{array}{l}\text { Bulweria } \\
\text { bulweril }\end{array}$ & & 4 & & - & & & 8 \\
\hline 1040 & $\begin{array}{l}\text { Bulweria } \\
\text { bulweril }\end{array}$ & & & & - & & & 3 \\
\hline 1042 & $\begin{array}{l}\text { Sterna } \\
\text { fuscata }\end{array}$ & & & & + & & & \\
\hline 1042 & $\begin{array}{l}\text { Sterna } \\
\text { fuscata }\end{array}$ & & & & - & & & \\
\hline
\end{tabular}


Wish remains indicated

by + or - sign p. 6 of 15

SOSC REF. NO. 229

POBSP INO. 10

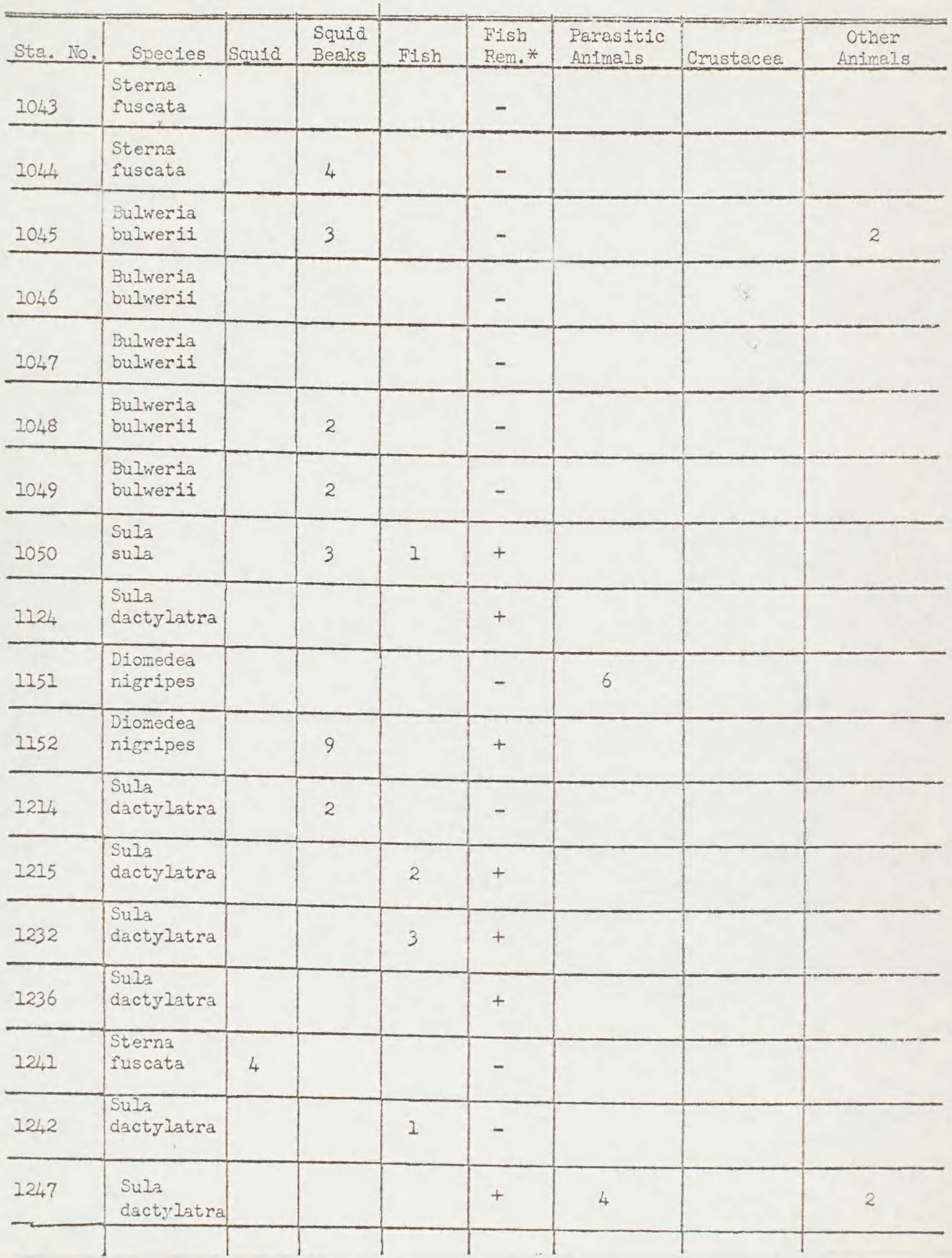


- *ish remains indicated

by + or - sign p. 7 of 15

SOSC REF. NO. 229

POBSP NO. 10

\begin{tabular}{|c|c|c|c|c|c|c|c|c|}
\hline Sta. No. & Species & Squid & $\begin{array}{l}\text { Squia } \\
\text { Beaks }\end{array}$ & Fish & $\begin{array}{l}\text { Fish } \\
\text { Rem.* }\end{array}$ & $\begin{array}{l}\text { Parasitic } \\
\text { Animals }\end{array}$ & Crustacea & $\begin{array}{c}\text { Other } \\
\text { Animals } \\
\end{array}$ \\
\hline 1256 & $\begin{array}{l}\text { Fregata } \\
\text { minor }\end{array}$ & & 32 & 2 & + & & & 3 \\
\hline 1261 & $\begin{array}{l}\text { Puffinus } \\
\text { pacificus }\end{array}$ & & 36 & & - & & & \\
\hline 1299 & $\begin{array}{l}\text { Sterna } \\
\text { fuscata }\end{array}$ & & & 2 & + & & & \\
\hline 1300 & $\begin{array}{l}\text { Sterna } \\
\text { fuscata }\end{array}$ & & 2 & & + & & & \\
\hline 1302 & $\begin{array}{l}\text { Sterna } \\
\text { fuscata }\end{array}$ & & & 2 & + & & & \\
\hline 1306 & $\begin{array}{l}\text { Puffinus } \\
\text { pacificus }\end{array}$ & 3 & 2 & & + & & & 1 \\
\hline 1308 & $\begin{array}{l}\text { Puffinus } \\
\text { pacificus }\end{array}$ & 5 & 57 & & + & & & 9 \\
\hline $13 u_{4}$ & $\begin{array}{l}\text { Fregata } \\
\text { minor }\end{array}$ & & 12 & & + & & & \\
\hline 2331 & $\begin{array}{l}\text { Puffinus } \\
\text { pacificus }\end{array}$ & 1 & & 4 & + & & & \\
\hline 1353 & $\begin{array}{l}\text { Puffinus } \\
\text { pacificus }\end{array}$ & 5 & 28 & 1 & - & & & 4 \\
\hline 1357 & $\begin{array}{l}\text { Phaethon } \\
\text { pubricauda }\end{array}$ & & 7 & & + & & & 1 \\
\hline 1358 & $\begin{array}{l}\text { Sterna } \\
\text { fuscata }\end{array}$ & 3 & & & + & & & \\
\hline 1359 & $\begin{array}{l}\text { Sterna } \\
\text { fuscata }\end{array}$ & 1 & & 1 & + & & & \\
\hline 1360 & $\begin{array}{l}\text { Sterna } \\
\text { Luscata }\end{array}$ & & 3 & & + & & & \\
\hline 1419 & $\begin{array}{l}\text { Pterodroma } \\
\text { externa }\end{array}$ & & 156 & 1 & + & & & \\
\hline 1420 & $\begin{array}{l}\text { Pterodroma } \\
\text { externa }\end{array}$ & & & & - & & & \\
\hline 1421 & $\begin{array}{l}\text { Pterodroma } \\
\text { externa }\end{array}$ & 6 & 46 & & - & & & 14 \\
\hline 1422 & $\begin{array}{l}\text { Sula } \\
\text { dactylatra }\end{array}$ & & & & + & & & \\
\hline & 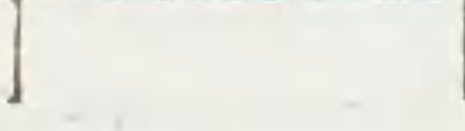 & & & & & & & \\
\hline
\end{tabular}


p. 8 of 15

*ish remains indicated by + or - sign

SOSC REF. NO. 229

POBSP NO. 10

\begin{tabular}{|c|c|c|c|c|c|c|c|c|}
\hline Sta. No. & Species & Squild & $\begin{array}{l}\text { Squid } \\
\text { Beaks } \\
\end{array}$ & Fish & $\begin{array}{l}\text { Fish } \\
\text { Rem.* }\end{array}$ & $\begin{array}{l}\text { Parasitic } \\
\text { Animals }\end{array}$ & Crustacea & $\begin{array}{l}\text { Other } \\
\text { Animals } \\
\end{array}$ \\
\hline 1423 & $\begin{array}{l}\text { Sterna } \\
\text { fuscata }\end{array}$ & & & & + & & & \\
\hline 1425 & $\begin{array}{l}\text { Pterodroma } \\
\text { externa }\end{array}$ & & 166 & & - & & & 4 \\
\hline 1426 & $\begin{array}{l}\text { Puffinus } \\
\text { pacificus }\end{array}$ & & 26 & & - & & & \\
\hline 1427 & $\begin{array}{l}\text { Puffinus } \\
\text { pacificus }\end{array}$ & & & & - & & & \\
\hline 1428 & $\begin{array}{l}\text { Puffinus } \\
\text { griseus }\end{array}$ & & 5 & & - & & & \\
\hline 1430 & $\begin{array}{l}\text { Puffinus } \\
\text { sp. }\end{array}$ & & & & - & 2 & & \\
\hline 1431 & $\begin{array}{l}\text { Pterodroma } \\
\text { externa }\end{array}$ & & 5 & & - & & & 1 \\
\hline 1432 & $\begin{array}{l}\text { Puffinus } \\
\text { pacificus }\end{array}$ & & & & + & & & \\
\hline 1433 & $\begin{array}{l}\text { Puffinus } \\
\text { tenuirostris }\end{array}$ & & & & - & & & \\
\hline 2434 & $\begin{array}{l}\text { Puffinus } \\
\text { tenuirostris }\end{array}$ & & & & - & & & \\
\hline 1435 & $\begin{array}{l}\text { Sterna } \\
\text { fuscata }\end{array}$ & (Sto & nach em & pty) & - & & & \\
\hline 1436 & $\begin{array}{l}\text { Puffinus } \\
\text { fenuirostrif }\end{array}$ & & & & - & & & \\
\hline 1437 & $\begin{array}{l}\text { Puffinus } \\
\text { tenuirost- } \\
\text { nis }\end{array}$ & & 8 & & - & & & \\
\hline 1438 & $\begin{array}{l}\text { Puffinus } \\
\text { pacificus }\end{array}$ & & 8 & & - & & & \\
\hline 1439 & $\begin{array}{l}\text { Sterna } \\
\text { fuscata. }\end{array}$ & & & & + & & & \\
\hline 1440 & $\begin{array}{l}\text { Puffinus } \\
\text { pacificus }\end{array}$ & & 7 & & - & & & \\
\hline 1441 & $\begin{array}{l}\text { Puffinus } \\
\text { tenuirost- } \\
\text { ris }\end{array}$ & & & & - & & & \\
\hline 1442 & $\begin{array}{l}\text { Puffinus } \\
\text { tenuiros- } \\
\text { ris }\end{array}$ & & & & - & & & \\
\hline & & & & & & & & \\
\hline
\end{tabular}


*Fish remains indicated by + or - sign p. 9 of 15

SOSC REF. NO. 229

POBSP NO. 20

\begin{tabular}{|c|c|c|c|c|c|c|c|c|}
\hline Sta. No. & Species & Squid & $\begin{array}{l}\text { Squia } \\
\text { Beaks } \\
\end{array}$ & Fish & $\begin{array}{l}\text { Fish } \\
\text { Rem.* }\end{array}$ & $\begin{array}{l}\text { Parasitic } \\
\text { Animals }\end{array}$ & Cmustacea & $\begin{array}{c}\text { Other } \\
\text { Animals }\end{array}$ \\
\hline 1443 & $\begin{array}{l}\text { Pluvialis } \\
\text { dominica }\end{array}$ & & & & - & & & \\
\hline 2444 & $\begin{array}{l}\text { Sterna } \\
\text { fuscata }\end{array}$ & & & & + & & & \\
\hline 11,45 & $\begin{array}{l}\text { Anous } \\
\text { stolidus }\end{array}$ & & & & - & 1 & & \\
\hline 1446 & $\begin{array}{l}\text { Anous } \\
\text { stolidus }\end{array}$ & (Ston & ach emply & 7) & - & & & \\
\hline 2448 & $\begin{array}{l}\text { Sula } \\
\text { dactylatra }\end{array}$ & & & 4 & + & & & \\
\hline 1449 & $\begin{array}{l}\text { Sterna } \\
\text { fuscata }\end{array}$ & & & 3 & + & & & \\
\hline 1450 & $\begin{array}{l}\text { Sula } \\
\text { dactylatra }\end{array}$ & & 21 & & + & 1 & & 2 \\
\hline 1451 & $\begin{array}{l}\text { Anous } \\
\text { stolidus }\end{array}$ & (Ston & ach empty & & & & & \\
\hline 1452 & \begin{tabular}{|l|} 
Sterna \\
fuscata
\end{tabular} & $\therefore 8$ & & & + & & & \\
\hline 1453 & $\begin{array}{l}\text { Arenaria } \\
\text { interpres }\end{array}$ & & & & - & & & \\
\hline 1454 & $\begin{array}{l}\text { Sterna } \\
\text { fuscata }\end{array}$ & & & & + & & & \\
\hline 1455 & $\begin{array}{l}\text { Sterna } \\
\text { fuscata }\end{array}$ & 2 & 4 & & + & & & 1 \\
\hline 1456 & $\begin{array}{l}\text { Puffinus } \\
\text { pacificus }\end{array}$ & 4 & 19 & & - & & & 1 \\
\hline 1457 & $\begin{array}{l}\text { Sterna } \\
\text { fuscata }\end{array}$ & & 4 & 1 & + & & & 2 \\
\hline 1458 & $\begin{array}{l}\text { Puffinus } \\
\text { pacificus }\end{array}$ & & 16 & & + & & & 6 \\
\hline 1459 & $\begin{array}{l}\text { Sula } \\
\text { sula }\end{array}$ & & 81 & & - & & & 10 \\
\hline 1460 & $\begin{array}{l}\text { Arenaria } \\
\text { interpres }\end{array}$ & (Stom & ch empty & & & & & \\
\hline 1461 & $\begin{array}{l}\text { Sula } \\
\text { sula }\end{array}$ & & & & + & & & \\
\hline
\end{tabular}


*Fish remains indicated

by + or - sign p. 10 of 15

SOSC REF. NO. 229

POBSP INO. 10

\begin{tabular}{|c|c|c|c|c|c|c|c|c|}
\hline Sta. No. & Species & Squid & $\begin{array}{l}\text { Squid } \\
\text { Beaks } \\
\end{array}$ & FIsh & $\begin{array}{l}\text { Fish } \\
\text { Rem.* }\end{array}$ & $\begin{array}{l}\text { Parasitic } \\
\text { Animals } \\
\end{array}$ & Crustacea & $\begin{array}{c}\text { Other } \\
\text { Animals } \\
\end{array}$ \\
\hline 1462 & $\begin{array}{l}\text { Pluvialis } \\
\text { dominica }\end{array}$ & & & & - & $x^{-10}$ & & \\
\hline 1463 & $\begin{array}{l}\text { Sterna } \\
\text { fuscata }\end{array}$ & & & & - & & & \\
\hline 1464 & $\begin{array}{l}\text { Sterna } \\
\text { fuscata }\end{array}$ & & 1 & & - & & & \\
\hline 1465 & $\begin{array}{l}\text { Sterna } \\
\text { fuscata }\end{array}$ & 3 & & & + & & & \\
\hline 1466 & $\begin{array}{l}\text { Sterna } \\
\text { fuscatia }\end{array}$ & 4 & & & - & & & \\
\hline 1467 & $\begin{array}{l}\text { Sterna } \\
\text { fuscata }\end{array}$ & 1 & 2 & 2 & + & & & \\
\hline 1468 & $\begin{array}{l}\text { Heteroscel- } \\
\text { us } \\
\text { incanum }\end{array}$ & (Sto) & nach ent & pty) & & & & \\
\hline 1469 & $\begin{array}{l}\text { Anous } \\
\text { stolidus }\end{array}$ & & & & - & & & \\
\hline 1470 & $\begin{array}{l}\text { Puffinus } \\
\text { tenuiros- } \\
\text { tris }\end{array}$ & & & & - & & & \\
\hline 1471 & $\begin{array}{l}\text { Pluvialis } \\
\text { dominica }\end{array}$ & & & & - & & & \\
\hline 2476 & $\begin{array}{l}\text { Sterna } \\
\text { fuscata }\end{array}$ & & & & - & & & \\
\hline 2478 & $\begin{array}{l}\text { Sterna } \\
\text { fuscata }\end{array}$ & (No : & \$pecimen & was rece & (ived) & & & \\
\hline 1482 & $\begin{array}{l}\text { Pterodroma } \\
\text { inexpectata }\end{array}$ & & 20 & & - & & & \\
\hline 4885 & $\begin{array}{l}\text { Oceanodroma } \\
\text { castro }\end{array}$ & & & & + & & & 1 \\
\hline 1487 & $\begin{array}{l}\text { Phaethon } \\
\text { lepturus }\end{array}$ & & & & + & & & \\
\hline 2488 & $\begin{array}{l}\text { Pluvialis } \\
\text { dominica }\end{array}$ & (No & pecimen & was rece & (ived.) & & & \\
\hline 1489 & $\begin{array}{l}\text { Oceanites } \\
\text { oceanicus }\end{array}$ & & 1 & & - & & & \\
\hline 2490 & $\begin{array}{l}\text { Oceanites } \\
\text { oceanicus }\end{array}$ & & & & + & & & \\
\hline
\end{tabular}


- *ish remains indicated

$b y+o r-s i g n$ p. 11 of 15

SOSC REF。 NO. 229

POBSP NO. 10

\begin{tabular}{|c|c|c|c|c|c|c|c|c|}
\hline Sta. No. & Species & Squia & $\begin{array}{l}\text { Squid } \\
\text { Beaks } \\
\end{array}$ & Fish & $\begin{array}{l}\text { Fish } \\
\text { Rem.** }\end{array}$ & $\begin{array}{l}\text { Parasitic } \\
\text { Animels }\end{array}$ & Cmustacea & $\begin{array}{c}\text { Other } \\
\text { Animals }\end{array}$ \\
\hline 1491 & $\begin{array}{l}\text { Oceanites } \\
\text { oceanicus }\end{array}$ & & & & - & & & \\
\hline 1494 & $\begin{array}{l}\text { Gygis } \\
\text { alba }\end{array}$ & & & & - & & & \\
\hline 1502 & $\left|\begin{array}{l}\text { Pt erodroma } \\
\text { inexpectata }\end{array}\right|$ & & 6 & & - & & & \\
\hline 1503 & $\begin{array}{l}\text { Pterodroma } \\
\text { inexpectata }\end{array}$ & & 2 & & + & & & \\
\hline 1510 & $\begin{array}{l}\text { Sterna } \\
\text { fuscata }\end{array}$ & & & & - & & & \\
\hline 1515 & $\begin{array}{l}\text { Oceanites } \\
\text { oceanicus }\end{array}$ & & & & + & & & \\
\hline 1519 & $\begin{array}{l}\text { Sterna } \\
\text { fuscata }\end{array}$ & & 1 & & - & & & \\
\hline 1521 & $\begin{array}{l}\text { Sterna } \\
\text { fuscata }\end{array}$ & 1 & 3 & 2 & - & & & \\
\hline 1533 & $\begin{array}{l}\text { Sula } \\
\text { sula }\end{array}$ & & 24 & & - & & & \\
\hline 1527 & $\begin{array}{l}\text { Phaethon } \\
\text { lepturus }\end{array}$ & & & & + & & & \\
\hline 1531 & $\mid \begin{array}{l}\text { Sterna } \\
\text { paradisaea }\end{array}$ & & & 4 & + & & & \\
\hline 1542 & $\begin{array}{l}\text { Sula } \\
\text { sula }\end{array}$ & 1 & 38 & & + & & & 9 \\
\hline 1547 & $\begin{array}{l}\text { Sula } \\
\text { dactylatra }\end{array}$ & & 1 & & - & 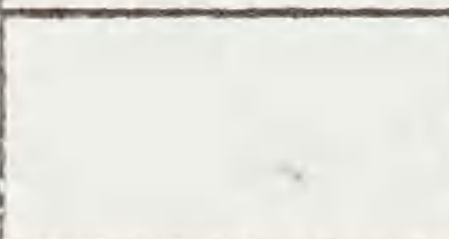 & & \\
\hline 1548 & $\begin{array}{l}\text { Sula } \\
\text { dactylatra }\end{array}$ & & & 2 & + & 1 & & \\
\hline 1551 & $\begin{array}{l}\text { Gygis } \\
\text { alba }\end{array}$ & (No & specimer & was rec & eived.) & & & \\
\hline 1552 & $\begin{array}{l}\text { Oceanodroma } \\
\text { leucorhoa }\end{array}$ & & 5 & & - & 1 & & 2 \\
\hline 1567 & $\begin{array}{l}\text { Oceanodroma } \\
\text { leucorhoa }\end{array}$ & & & & - & & & \\
\hline 1570 & $\begin{array}{l}\text { Pterodroma } \\
\text { alba }\end{array}$ & & 180 & & - & & & \\
\hline & & & & & & & & \\
\hline
\end{tabular}


*ish remains indicated

p. 12 of 15

by + or - sign

SOSC REF. NO. 229

POBSP INO. 10

\begin{tabular}{|c|c|c|c|c|c|c|c|c|}
\hline Sta. No. & Species & Squid & $\begin{array}{l}\text { Squia } \\
\text { Beaks } \\
\end{array}$ & Fish & $\begin{array}{l}\text { Fish } \\
\text { Rem.* }\end{array}$ & $\begin{array}{l}\text { Parasitic } \\
\text { Animals }\end{array}$ & Crustacea & $\begin{array}{c}\text { Other } \\
\text { Animals }\end{array}$ \\
\hline 1571 & $\begin{array}{l}\text { Pt erodroma } \\
\text { externa }\end{array}$ & 1 & 7 & 2 & + & & & 1 \\
\hline 1572 & $\begin{array}{l}\text { Pterodroma } \\
\text { hypoleuca }\end{array}$ & & 9 & & - & & & 13 \\
\hline 1573 & $\begin{array}{l}\text { Pterodroma } \\
\text { externa }\end{array}$ & & & & - & & & \\
\hline 1574 & $\begin{array}{l}\text { Pterodroma } \\
\text { hypoleuca }\end{array}$ & & 10 & & - & & & \\
\hline 1575 & $\begin{array}{l}\text { Pterodroma } \\
\text { hypoleuca }\end{array}$ & & & & - & 1 & & \\
\hline 1576 & $\begin{array}{l}\text { Oceanodroma } \\
\text { leucorhoa }\end{array}$ & & & & - & & & \\
\hline 5592 & $\begin{array}{l}\text { Anous } \\
\text { stolidus }\end{array}$ & (No) & specime & was rec & eived) & & & \\
\hline 5756 & $\begin{array}{l}\text { Sula } \\
\text { dactylatra }\end{array}$ & & 3 & & - & & $\therefore$ & \\
\hline 5758 & $\begin{array}{l}\text { Anous } \\
\text { minutus }\end{array}$ & & & & - & & st & \\
\hline 5760 & $\begin{array}{l}\text { Sula } \\
\text { sula }\end{array}$ & 5 & & & + & & & \\
\hline 5764 & $\begin{array}{l}\text { Phaethon } \\
\text { rubricauda }\end{array}$ & & 10 & & - & & & \\
\hline 5765 & $\begin{array}{l}\text { Fregata } \\
\text { ariel }\end{array}$ & & 24 & & - & & & 9 \\
\hline 5766 & $\begin{array}{l}\text { Sula } \\
\text { dactylatra }\end{array}$ & & 76 & & - & 1 & & \\
\hline 5767 & $\begin{array}{l}\text { Sula } \\
\text { dactylatra }\end{array}$ & & 47 & & - & & & 6 \\
\hline 5768 & $\begin{array}{l}\text { Puffinus } \\
\text { pacificus }\end{array}$ & & & & - & & & \\
\hline 5769 & $\begin{array}{l}\text { Heteroscelus } \\
\text { incanum }\end{array}$ & & & & - & & & \\
\hline 5770 & $\begin{array}{l}\text { Erolia } \\
\text { melanotos }\end{array}$ & & & & - & & & \\
\hline 5771 & $\begin{array}{l}\text { Sula } \\
\text { leucogaster }\end{array}$ & & 2 & & - & & & \\
\hline
\end{tabular}


- wish remains indicated by + or - sign p. 13 of 15

SOSC REF. NO. 229

POBSP NO. 10

\begin{tabular}{|c|c|c|c|c|c|c|c|c|}
\hline Sta. No. & Species & Squid & $\begin{array}{l}\text { Squida } \\
\text { Beaks }\end{array}$ & Fish & $\begin{array}{l}\text { Fish } \\
\text { Rem.* }\end{array}$ & $\begin{array}{l}\text { Parasitic } \\
\text { Animals }\end{array}$ & Crustacea & $\begin{array}{c}\text { Other } \\
\text { Animals }\end{array}$ \\
\hline 5806 & $\begin{array}{l}\text { Sula } \\
\text { dactylatra }\end{array}$ & & & 2 & - & & & \\
\hline 5807 & $\begin{array}{l}\text { Erolia } \\
\text { melanotos }\end{array}$ & & & & - & & & \\
\hline 5808 & $\begin{array}{l}\text { Erolia } \\
\text { melanotos }\end{array}$ & & & & - & & & \\
\hline 5809 & $\begin{array}{l}\text { Sula } \\
\text { sula }\end{array}$ & & 7 & & + & & & 2 \\
\hline 5811 & $\begin{array}{l}\text { Sterna } \\
\text { fuscata }\end{array}$ & & & & + & & & \\
\hline 5812 & $\begin{array}{l}\text { Sterna } \\
\text { fuscata }\end{array}$ & (Stc & nach emp & ty) & & & & \\
\hline 5814 & $\begin{array}{l}\text { Erolia } \\
\text { acuminata }\end{array}$ & (Sto & lach emp & $t y)$ & & & & \\
\hline 5815 & $\begin{array}{l}\text { Numenius } \\
\text { tahitiensis }\end{array}$ & & & & - & & & \\
\hline 5816 & $\begin{array}{l}\text { Heteroscelus } \\
\text { incanum }\end{array}$ & & & & - & & 9 & \\
\hline 5818 & $\begin{array}{l}\text { Limosa } \\
\text { lapponica }\end{array}$ & & & & + & & & \\
\hline 5819 & $\begin{array}{l}\text { Erolia } \\
\text { acuminata }\end{array}$ & & & & - & & & \\
\hline 5820 & $\begin{array}{l}\begin{array}{l}\text { Vini } \\
\text { kuhli }\end{array} \\
\end{array}$ & & & & - & & & \\
\hline 5821 & $\begin{array}{l}\text { Thalasseus } \\
\text { bergit }\end{array}$ & & & & + & & & \\
\hline 5822 & $\begin{array}{l}\text { Vini } \\
\text { kuhli }\end{array}$ & & & & - & & & \\
\hline 5823 & $\begin{array}{l}\text { Gygis } \\
\text { alba }\end{array}$ & & 1 & & - & & 1 & \\
\hline 5824 & $\begin{array}{l}\text { Thalasseus } \\
\text { bergii }\end{array}$ & & & 1 & + & & & \\
\hline 5825 & $\begin{array}{l}\text { Thalasseus } \\
\text { bergii }\end{array}$ & (Ston & ach empt & & & & & \\
\hline 5826 & $\begin{array}{l}\text { PIuvialis } \\
\text { dominica }\end{array}$ & & & & - & & & \\
\hline & & & & & & & & \\
\hline
\end{tabular}


Wish remains indicated

p. 14 of 15

by + or - sign

SOSC REF. NO. 229

POBSP NO. 10

\begin{tabular}{|c|c|c|c|c|c|c|c|c|}
\hline Sta. No. & Species & Squid & $\begin{array}{l}\text { Squid } \\
\text { Beaks } \\
\end{array}$ & Fish & $\begin{array}{l}\text { Fish } \\
\text { Rem.* }\end{array}$ & $\begin{array}{l}\text { Parasitic } \\
\text { Anlmals }\end{array}$ & Crustacea & $\begin{array}{c}\text { Other } \\
\text { Animals } \\
\end{array}$ \\
\hline 5827 & $\begin{array}{l}\text { Pluvialis } \\
\text { dominica }\end{array}$ & & & & - & & & \\
\hline 5828 & $\begin{array}{l}\text { Numenius } \\
\text { tahitiensis }\end{array}$ & & & & - & & & \\
\hline 5829 & $\begin{array}{l}\text { Numenius } \\
\text { tahitiensis }\end{array}$ & & & & - & & & \\
\hline 5831 & $\begin{array}{l}\text { Sula } \\
\text { dactylatra }\end{array}$ & & 23 & & - & & & 7 \\
\hline 5833 & $\begin{array}{l}\text { Spatula } \\
\text { clypeata }\end{array}$ & & & & - & & & 1 \\
\hline 5863 & $\begin{array}{l}\text { Pterodroma } \\
\text { alba }\end{array}$ & 3 & & & - & & & 4 \\
\hline 5864 & $\begin{array}{l}\text { Pterodroma } \\
\text { alba }\end{array}$ & & 6 & & - & & & \\
\hline 5865 & $\begin{array}{l}\text { Pterodroma } \\
\text { alba }\end{array}$ & & 27 & & - & & & \\
\hline 5867 & $\begin{array}{l}\text { Sula } \\
\text { sula }\end{array}$ & & & & - & & & 2 \\
\hline 20282 & $\begin{array}{l}\text { Totanus } \\
\text { flavipes }\end{array}$ & & & & - & & & \\
\hline 20289 & $\begin{array}{l}\text { Sula } \\
\text { sula }\end{array}$ & & & & - & & & \\
\hline 20440 & $\begin{array}{l}\text { Gygis } \\
\text { alba }\end{array}$ & (Ston & ach empt & y) & - & & & \\
\hline 20477 & $\begin{array}{l}\text { Phaethon } \\
\text { rubricauda }\end{array}$ & & 8 & & - & 4 & . & \\
\hline 20478 & $\begin{array}{l}\text { Sula } \\
\text { sula }\end{array}$ & & & & + & & & \\
\hline 20481 & $\begin{array}{l}\text { Fregata } \\
\text { minor }\end{array}$ & & 3 & & + & & & \\
\hline 20482 & $\begin{array}{l}\text { Fregata } \\
\text { minor }\end{array}$ & & 4 & 1 & + & 1 & & 5 \\
\hline 20485 & $\begin{array}{l}\text { Phaethon } \\
\text { rubricauda }\end{array}$ & & 32 & & - & 1 & & \\
\hline 30031 & $\begin{array}{l}\text { Arenaria } \\
\text { interpres }\end{array}$ & & & & - & & & \\
\hline
\end{tabular}


*Fish remains indicated

p. 15 of 15

by + or - sign

SOSC REF. NO. 229

POBSP NO. 10

\begin{tabular}{|c|c|c|c|c|c|c|c|c|}
\hline Sta. No. & Species & Squid & $\begin{array}{l}\text { Squid } \\
\text { Beaks } \\
\end{array}$ & Fish & $\begin{array}{l}\text { Fish } \\
\text { Rem.* }\end{array}$ & $\begin{array}{l}\text { Parasitic } \\
\text { Animals }\end{array}$ & Crustacea & $\begin{array}{c}\text { Other } \\
\text { Animals }\end{array}$ \\
\hline 30033 & $\begin{array}{l}\text { Arenaria } \\
\text { interpres }\end{array}$ & & & & - & & & \\
\hline 15008 & $\begin{array}{l}\text { Mimus } \\
\text { polyglotto\$ }\end{array}$ & & & & - & & & \\
\hline TOTAL & 254 & 89 & 2032 & 89 & 109 & 46 & 10 & 202 \\
\hline
\end{tabular}

